\title{
Photonic Vector Processing Techniques for Radiofrequency Signals
}

Miguel Ángel PiQueras RuipéReZ

SUPERVISORS:

DR. JUAN LUIS CORRAL GONZÁLEZ DR. BORJA VIDAL RODRÍGUEZ
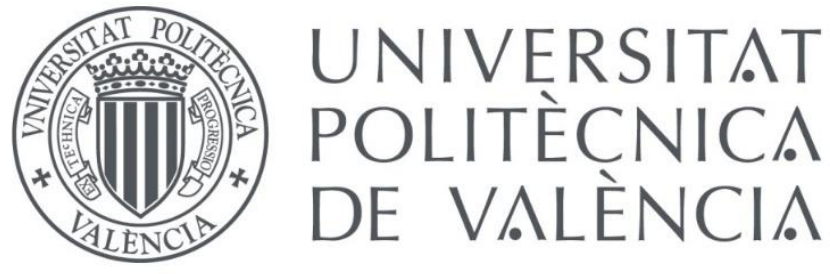

Departamento de Comunicaciones UniVERSIDAd Politécnica de VAlenCia Valencia, December 2015 


\section{Abstract}

The processing of radiofrequency signals using photonics means is a discipline that appeared almost at the same time as the laser and the optical fibre. Photonics offers the capability of managing broadband radiofrequency (RF) signals thanks to its low transmission attenuation, a variety of linear and non-linear phenomena and, recently, the potential to implement integrated photonic subsystems. These features open the door for the implementation of multiple functionalities including optical transportation, up and down frequency conversion, optical RF filtering, signal multiplexing, de-multiplexing, routing and switching, optical sampling, tone generation, delay control, beamforming and photonic generation of digital modulations, and even a combination of several of these functionalities. This thesis is focused on the application of vector processing in the optical domain to radiofrequency signals in two fields of application: optical beamforming, and photonic vector modulation and demodulation of digital quadrature amplitude modulations. The photonic vector control enables to adjust the amplitude and phase of the radiofrequency signals in the optical domain, which is the fundamental processing that is required in different applications such as beamforming networks for direct radiating array (DRA) antennas and multilevel quadrature modulation.

DRAs consist of a set of individual antenna elements working simultaneously as an array antenna with an equivalent electrical size of a bigger single antenna. All the antenna elements radiate the same signal and, when all this signals interfere in the far-field, a constructive interference is achieved at a certain angular direction. Conventional implementations in the digital or microwave domain can show a high degree of complexity of the beamforming networks (i.e. the subsystem that performs the signal splitting and the phase and amplitude control) since it increases dramatically with the size of the antenna impacting in their size, mass, volume, 
consumption and reliability. Alternative technologies, such as these based on photonics, offer potential to circumvent these drawbacks.

The work described in this thesis include different techniques for implementing a photonic version of beamforming networks for direct radiating arrays (DRA) known as optical beamforming networks (OBFN), with the objectives of providing a precise control in terrestrial applications of broadband signals at very high frequencies above $40 \mathrm{GHz}$ in communication antennas, optimizing the size and mass when compared with the electrical counterparts in space application, and presenting new photonic-based OBFN functionalities. Thus, two families of OBFNs are studied: fibre-based true time-delay architectures and integrated networks. The first allow the control of broadband signals using dispersive optical fibres with wavelength division multiplexing techniques and advanced functionalities such as direction of arrival estimation in receiving architectures. In the second, passive OBFNs based on monolithically-integrated Optical Butler Matrices are studied, including an ultra-compact solution using optical heterodyne techniques in siliconon-insulator (SOI) material, and an alternative implementing a homodyne counterpart in germanium-doped silica material.

In this thesis, the application of photonic vector processing to the generation of quadrature digital modulations has also been investigated. Multilevel modulations are based on encoding digital information in discrete states of phase and amplitude of an electrical signal to enhance spectral efficiency, as for instance, in quadrature modulation. The signal process required for generating and demodulating this kind of signals involves vector processing (phase and amplitude control) and frequency conversion. Unlike the common electronic or digital implementation, in this thesis, different photonic-based signal processing techniques are studied to produce digital modulation (photonic vector modulation, PVM) and demodulation (PVdM). These techniques are of particular interest in the case of broadband signals where the data-rate required to be managed is in the order of gigabit per second, for applications like wireless backhauling of metro optical networks (known as fibre-to-the-air). The techniques described use optical dispersion in optical fibres, wavelength division multiplexing and photonic up/down conversion. Additionally, an optical heterodyne solution implemented monolithically in a photonic integrated circuit (PIC) is also described. 
El procesamiento de señales de radiofrecuencia (RF) utilizando medios fotónicos es una disciplina que apareció casial mismo tiempo que el láser y la fibra óptica. La fotónica ofrece la capacidad de manipular señales de radiofrecuencia de banda ancha, una baja atenuación, procesados basados en una amplia variedad de fenómenos lineales y no lineales y, recientemente, el potencial para implementar subsistemas fotónicos integrados. Estas características ofrecen un gran potencial para la implementación de múltiples funcionalidades incluyendo transporte óptico, conversión de frecuencia, filtrado óptico de RF, multiplexación y demultiplexación de señales, encaminamiento y conmutación, muestreo óptico, generación de tonos, líneas de retardo, conformación de haz en agrupaciones de antenas o generación fotónica de modulaciones digitales, e incluso una combinación de varias de estas funcionalidades. Esta tesis se centra en la aplicación del procesamiento vectorial en el dominio óptico de señales de radiofrecuencia en dos campos de aplicación: la conformación óptica de haces y la modulación y demodulación vectorial fotónica de señales digitales en cuadratura. El control fotónico vectorial permite manipular la amplitud y fase de las señales de radiofrecuencia en el dominio óptico, que es el procesamiento fundamental que se requiere en diferentes aplicaciones tales como las redes de conformación de haces para agrupaciones de antenas y en la modulación en cuadratura. 
Las agrupaciones de antenas consisten en un conjunto de antenas individuales que trabajan al unísono como un único elemento radiante de mayor tamaño. Todos los elementos de la agrupación irradian la misma señal y, cuando todas las señales interfieren en campo lejano, se produce una interferencia constructiva en una cierta dirección angular. Las implementaciones convencionales en el dominio digital o microondas pueden mostrar un alto grado de complejidad de las redes de conformación de haces (es decir, de los subsistemas que realizan la división de la señal y el control de fase, y, también el control de la amplitud) incrementándose dramáticamente su tamaño, peso, volumen, consumo y fiabilidad al aumentar el número de elementos. Las tecnologías alternativas, tales como las basadas en la fotónica, al basarse en una plataforma distinta ofrecen el potencial para eludir estos inconvenientes.

El trabajo descrito en esta tesis incluye diferentes técnicas para implementar una versión fotónica de las redes de conformación de haces de en agrupaciones de antenas, conocidas como redes ópticas de conformación de haces (OBFN), con los objetivos de proporcionar un control preciso en aplicaciones terrestres de señales de banda ancha a frecuencias muy altas por encima de $40 \mathrm{GHz}$ en antenas de comunicaciones, optimizando el tamaño y el peso cuando se compara con los homólogos eléctricos en aplicaciones espaciales, y la presentación de nuevas funcionalidades fotónicas para agrupaciones de antenas. Por lo tanto, se estudian dos familias de OBFNs: arquitecturas de retardo en fibra óptica y arquitecturas integradas. Las primeras permiten el control de señales de banda ancha utilizando fibras ópticas dispersivas con técnicas de multiplexado por división de longitud de onda y funcionalidades avanzadas tales como la estimación del ángulo de llegada de la señal en la antena receptora. En la segunda, se estudian redes de conformación pasivas basadas en Matrices de Butler ópticas integradas, incluyendo una solución ultra-compacta utilizando técnicas ópticas heterodinas en silicio sobre aislante (SOI), y una alternativa homodina en silice dopado con germanio.

En esta tesis, también se han investigado técnicas de procesado vectorial fotónico para la generación de modulaciones digitales en cuadratura. Las modulaciones multinivel codifican la información digital en estados discretos de fase y amplitud de una señal eléctrica para aumentar su eficiencia espectral, como por ejemplo la modulación en cuadratura. El procesado necesario para generar y demodular este tipo de señales implica el procesamiento vectorial (control de amplitud y fase) y la conversión de frecuencia. A diferencia de la implementación electrónica o digital convencional, en esta tesis se estudian diferentes técnicas de procesado fotónico tanto para la generación de modulaciones digitales (modulación vectorial fotónica, $\mathrm{PVM}$ ) como para su demodulación (PVdM). Esto es de particular interés en el caso de señales de banda ancha, donde la velocidad de datos requerida es del orden de gigabits por segundo, para aplicaciones como backhaul inalámbrico de redes ópticas metropolitanas (conocida como fibra hasta el aire). Las técnicas descritas se basan 
en explotar la dispersión cromática de la fibra óptica, la multiplexación por división de longitud de onda y la conversión en frecuencia. Además, se pre senta una solución heterodina implementada monolíticamente en un circuito integrado fotónico (PIC). 
El processament de senyals de radiofreqüència $(\mathrm{RF})$ utilitzant mitjans fotònics és una disciplina que va aparèixer gairebé al mateix temps que el làser i la fibra òptica. La fotònica ofereix la capacitat de manipular senyals de radiofreqüència de banda ampla, una baixa atenuació, processats basats en una àmplia varietat de fenòmens lineals i no lineals i, recentment, el potencial per implementar subsistemes fotònics integrats. Aquestes característiques ofereixen un gran potencial per a la implementació de múltiples funcionalitats incloent transport òptic, conversió de freqüència, filtrat òptic de RF, multiplexació i demultiplexació de senyals, encaminament i commutació, mostreig òptic, generació de tons, línies de retard, conformació de feix en agrupacions d'antenes i la generació fotònica de modulacions digitals, i fins i tot una combinació de diverses d'aquestes funcionalitats. Aquesta tesi es centra en l'aplicació del processament vectorial en el domini òptic de senyals de radiofreqüència en dos camps d'aplicació: la conformació òptica de feixos i la modulació i demodulació vectorial fotònica de senyals digitals en quadratura. El control fotònic vectorial permet manipular l'amplitud i la fase dels senyals de radiofreqüència en el domini òptic, que és el processament fonamental que es requereix en diferents aplicacions com ara les xarxes de conformació de feixos per agrupacions d'antenes i en modulació multinivell.

Les agrupacions d'antenes consisteixen en un conjunt d'antenes individuals que treballen a l'uníson com un únic element radiant de major grandària. Tots els 
elements de l'agrupació irradien el mateix senyal i, quan tots els senyals interfereixen en camp llunyà, es produeix una interferència constructiva en una certa direcció angular. Les implementacions convencionals en el domini digital o microones poden mostrar un alt grau de complexitat de les xarxes de conformació de feixos (és a dir, dels subsistemes que realitzen la divisió del senyal i el control de fase, i, també el control de l'amplitud) incrementant dramàticament la seua grandària, pes, volum, consum i fiabilitat amb l'increment del nombre d'elements. Les tecnologies alternatives, com ara les basades en la fotònica, en basar-se en una plataforma diferent ofereixen el potencial per eludir aquests inconvenients.

El treball descrit en aquesta tesi inclou diferents tècniques per implementar una versió fotònica de les xarxes de conformació de feixos en agrupacions d'antenes, conegudes com a xarxes òptiques de conformació de feixos (OBFN), amb els objectius de proporcionar un control precís en aplicacions terrestres de senyals de banda ampla a freqüències molt altes per sobre de $40 \mathrm{GHz}$ en antenes de comunicacions, optimitzant la mida i el pes quan es compara amb els homòlegs elèctrics en aplicacions espacials, i la presentació de noves funcionalitats fotòniques per agrupacions d'antenes. Per tant, s'estudien dues famílies de OBFNs: arquitectures de retard en fibra òptica i arquitectures integrades. Les primeres permeten el control de senyals de banda ampla utilitzant fibres òptiques dispersives amb tècniques de multiplexació per divisió en longitud d'ona i funcionalitats avançades com ara l'estimació de l'angle d'arribada del senyal a l'antena receptora. A la segona, s'estudien xarxes de conformació passives basades en Matrius de Butler òptiques en fotònica integrada, incloent una solució ultra-compacta utilitzant tècniques òptiques heterodinas en silici sobre aillant (SOI), i una alternativa homodina en silice dopat amb germani.

D'altra banda, també s'ha investigat en aquesta tesi tècniques de processament vectorial fotònic per a la generació de modulacions digitals en quadratura. Les modulacions multinivell codifiquen la informació digital en estats discrets de fase i amplitud d'un senyal elèctric per augmentar la seva eficiència espectral, com ara la modulació en quadratura. El processat necessari per generar i desmodular aquest tipus de senyals implica el processament vectorial (control d'amplitud i fase) i la conversió de freqüència. A diferència de la implementació electrònica o digital convencional, en aquesta tesi s'estudien diferents tècniques de processament fotònic tant per a la generació de modulacions digitals (modulació vectorial fotònica, PVM) com per la seva demodulació ( $\mathrm{PVdM})$. Això és de particular interès en el cas de senyals de banda ampla, on la velocitat de dades requerida és de l'ordre de gigabits per segon, per a aplicacions com backhaul sense fils de xarxes òptiques metropolitanes (coneguda com fibra fins l'aire). Les tècniques descrites es basen en explotar la dispersió cromàtica de la fibra òptica, la multiplexació per divisió en longitud d'ona i la conversió en freqüència. A més, es presenta una solució heterodina implementada monolíticament en un circuit integrat fotònic (PIC). 


\section{List of publications included in the Thesis}

The following papers have been derived from the work of the Thesis:

\section{PUBLICATIONS IN INTERNATIONAL JOURNALS}

A. M. A. Piqueras, B. Vidal, J. Herrera, V. Polo, J. L. Corral, J. Martí, "Photonic switched beamformer implementation for broadband wireless access in transmission and reception modes at $42.7 \mathrm{GHz}$ ", Optics Communications, vol. 249, Issue 4-6, pp. 441-449, May 2005.

B. M. A. Pique ras, G. Grosskopf, B. Vidal, J. Herrera, J. M. Martínez, P. Sanchis, V. Polo, J. L. Corral, A. Marceaux, J. Galière, J. Lopez, A. Enard, J-L. Valard, O. Parillaud, E. Estèbe, N. Vodjdani, M-S. Choi, J. H. den Besten, F. M. Soares, M. K. Smit, and J. Marti, "Optically Beamformed Beam-Switched Adaptive Antennas for Fixed and Mobile Broadband Wireless Access Networks", (Invited) IEEE Transactions On Microwave Theory And Techniques, vol. 45, no. 2, pp. 887-899, February 2006.

C. B. Vidal, M. A. Pique ras, J. Martí, "Multibeam photonic beamformer based on opticalfilters", Electronics Letters, vol. 42, no. 17, pp. 980-981, August 2006. 
D. B. Vidal, M. A. Piqueras, J. Martí, "Direction of Arrival Estimation of Broadband Microwave Signals in Phased Array Antennas using Photonic Technique", IEEE Journal of Lightwave Technology, vol. 24, no. 7, January 2006.

E. P. Sanchis, J. V. Galán, A. Griol, J. Martí, M.A. Pique ras, J. M. Perdigués, "Low-crosstalk in silicon-on-insulator waveguide crossings with optimizedangle”, IEEE Photonics Technology Letters, vol. 19, pp. 1583-1585, 2007.

F. M. A. Pique ras, B. Vidal, J. L. Corral, V. Polo, A. Martínez, J. Martí, “Direct Photonic Generation of Electrical Vector Modulations at Microwavel Millimeter-wave Frequencies", IEEE Photonic Technology Letters, vol. 17, no. 9, pp. 1947-1949, September 2005.

\section{CONTRIBUTIONS TO INTERNATIONAL CONGRESS}

G. B. Vidal, M. A. Piqueras, J. Herrera, V. Polo, J. L. Corral, J. Martí, "Radiation pattern measurements and 155 Mbps 32-QAM indoor data transmission at 42.7 GHz using a photonic beamformed beam-switched antenna based on optical switches and dispersive media", IST Mobile \& Wireless Telecommunications Summit 2004, Lyon (France), 27-30 June, 2004.

H. M. A. Piqueras, B. Vidal, J. Herrera, V. Polo, J. L. Corral, J. Martí, “Optical Switched Beamformer for Broadband Wireless Access Networks at $40 \mathrm{GHz}$ in Receiving Mode", 30 ${ }^{\text {th }}$ European Conference on Optical Communication (ECOC), Stockholm (Sweden), 5-9 September 2004.

I. B. Vidal, M. A. Piqueras, J. Herrera, V. Polo, J. L. Corral, J. Martí, "Experimental Demonstration of a 3-bit Photonic Beamformer at the mm-band in Transmission and Reception Modes", 2004 International Topical Meeting on Microwave Photonics (MWP2004), Ogunquit (USA), 4-6 October 2004.

J. M. A. Piqueras, S. De la Rosa, D. Zorrilla, J. Martí, G. Caille, J.M. Perdigués, P. Sanchis, A. Griol, B. Sanchez, G. Sanchez, J. V. Galán, A. Brimont, J. Hurtado, J. Ayucar, "Nanophotonic technology in space for beamforming applications", 6th ESA Round Table on Micro \& Nano Technologies for Space Applications, ESA/ESTEC, Noordwijk (The Netherlands), 8-12 October 2007. 
K. P. Sanchis, J. V. Galán, A. Brimont, A. Griol, J. Martí, M. A. Piqueras, J. M. Perdigués, "Low-crosstalk in silicon-on-insulator waveguide crossings with optimized-angle”, $4^{\text {th }}$ Group Four Photonics Conference, Paper WP38, pp.159161, Tokyo (Japan), 2007.

L. M. A. Piqueras, T. Mengual, O. Navasquillo, M. Sotom, G. Caille, "OptoMicrowave, Butler matrices based Front-End for a Multi-Beam large Direct Radiating Array Antenna", International Conference on Space Optics, Tenerife (Spain), 7-10 October 2014.

M. M. A. Piqueras, B. Vidal, J. L. Corral, V. Polo, H. Pfrommer, A. Martínez and J. Martí, "Photonic Vector Modulation Tx/Rx Architecture for Generation, Remote Delivery and Detection of M-QAM Signals ", IEEE MTT-S International Microwave Symposium 2005, Long Beach (USA), 12-15 June 2005.

N. M. A. Piqueras, B. Vidal, J. L. Corral, A. Martínez, J. Martí, "Photonic Vector Demodulation Architecture for Remote Detection of M-QAM Signals”, 2005 International Topical Meeting on Microwave Photonics (MWP 2005), Seoul (Korea), October 12-14, 2005.

O. J. Martí, M.A. Piqueras, B. Vidal, H. Pfrommer, V. Polo, A. Ramírez, D. Zorrilla, "Radio-over-Fiber Multi-Service MM-Wave Interconnection with Photonic Up-conversion, Dual Band Remote Delivery and Photonic Envelope Detection", WFC-5 IEEE MTT-S International Microwave Symposium, San Francisco (USA), June 11-16, 2006.

P. M. A. Piqueras, V. Polo, J. L. Corral, B. Vidal, A. Martínez, J. Martí, "RadioOver-Fibre Broadband Quadrature Amplitude Modulator", 32 ${ }^{\text {nd }}$ European Conference on Optical Communications (ECOC), Cannes (France), September 24-28, 2006.

Q. M. A. Piqueras, V. Polo, J. Martí, "Simultaneous base-band and mm-wave delivery of Gb/s data employing photonic vector modulators", Optical Fiber Communication Conference (OFC), , JThA60, Anaheim (USA), March 2007.

R. J. L. Corral, R. Sambaraju, M. A. Piqueras, V. Polo, "Pure QAM Signal Generation with Photonic Vector Modulator", Optical Fiber Communication Conference (OFC), San Diego (USA), 2008. 


\section{PATENTS}

S. M. A. Piqueras, "Sistema y método de conformación óptica de haces", Spain P200703407, Issued December 21 ${ }^{\text {st }}, 2007$

T. G. Caille, M. Sotom, M. A. Pique ras, T. Mengual, "Distributed feeding circuit for antenna beamforming array", US Patent No. US2014/0320346A1, Publication date: October 30, 2014.

a. Extended to Europe (EP2797167A1), Canada (CA3849852A1), Japan (JP2014217066A), France (FR3005210A1)

U. A. Hakansson, M. A. Pique ras, R. Sambaraju, "Enlaces inalámbricos digitales con modulación de fase multinivel basados en fotónica", Spain ES 2334759 B1. Issued February $2^{\text {nd }}, 2011$. 


\section{Table of Contents}

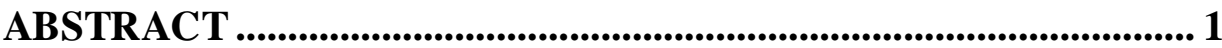

LIST OF PUBLICATIONS INCLUDED IN THE THESIS .............. 8

PUB LICATIONS IN INTERNATIONAL JOURNALS.................................................... 8

CONTRIB UTIONS TO INTERNATIONAL CONGRESS..............................................9

PATENTS …..............................................................................................................................11

1. INTRODUCTION TO PHOTONIC TECHNOLOGY .............. 1

1.1. Fibre-optic Systems ........................................................................................................................ 2

1.2. Photonic Integrated Circuits (PIC) ................................................................................6

1.3. Microwave Photonics..................................................................................................................... 8

1.4. Microwave Photonic Links...................................................................................................10

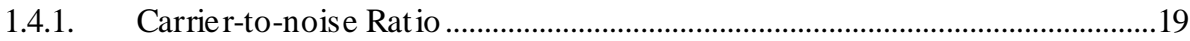

1.4.1.1. Thermal noise …………………………………………………………. 20

1.4.1.2. Shot noise

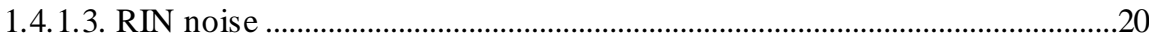

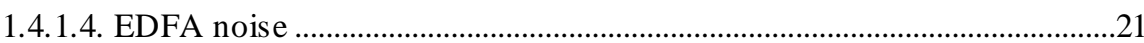

1.5. Photonic Up and Down Conversion .............................................................................23

1.6. Vector Microwave Optical Processing ...........................................................................25

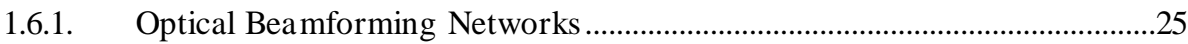

1.6.2. Photonic Vector Modulator for Co mmunications ............................................27

2. OPTICAL BEAMFORMING NETWORKS ......................... 29

2.1. Introduction to Antenna Theory ................................................................................29

2.1.1. Scanning and Collimation of Linear and Planar Arrays ..................................30

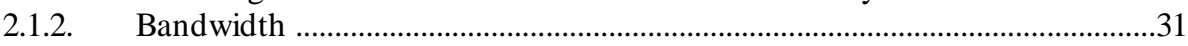

2.1.3. Time-Delay Compensation..................................................................................32

2.1.4. Multiple Beam Array Feeds .............................................................................34

2.1.5. Control for Wideband Arrays...........................................................................37

2.2. Review of Optical Beamforming Networks ..............................................................39 


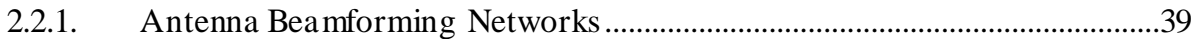

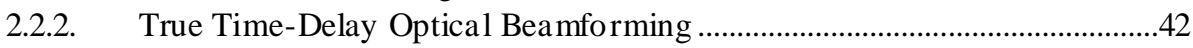

2.2.2.1. Free-space and semi-guided-wave optical delay line bea mformers ............42

2.2.2.2. Fib re-optic delay line beamformers ..................................................................44

2.2.2.3. WDM beamformers based on Dispersion Fibre Prism, Fibre Bragg

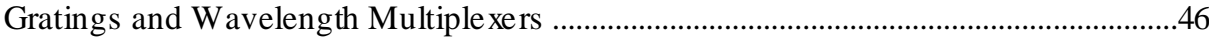

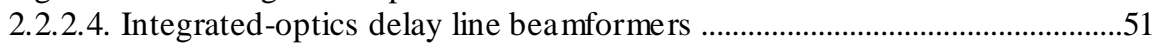

2.2.2.5. Phase-shifting coherent optical beamformers ................................................52

2.2.2.6. Free-s pace Fourier trans form optical signal proces sors (OSP) ....................53

2.2.2.7. Hybrid SLM-based coherent beamformers ..................................................53

2.2.2.8. Optical coherent beamformer based on integrated phase-shifters ................54

2.3. Conclusion

\section{PHOTONIC SWITCHED BEAMFORMER FOR} BROADBAND WIRELESS ACCESS ........................................................ 60

3.1. Beamfor mer Architecture Descri ption .........................................................................661

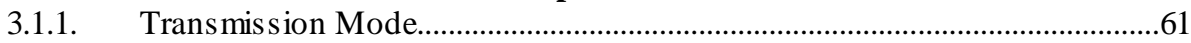

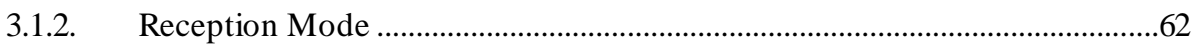

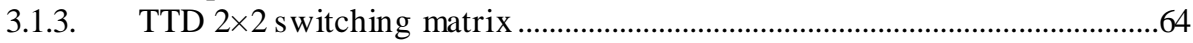

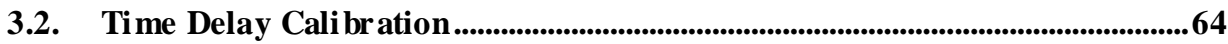

3.3. System Performance Anal ysis .........................................................................66

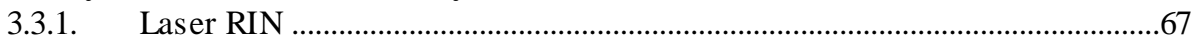

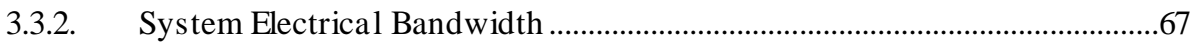

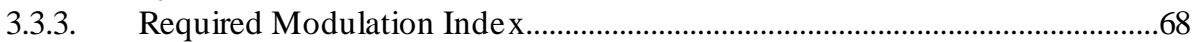

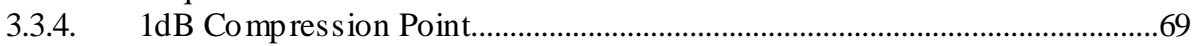

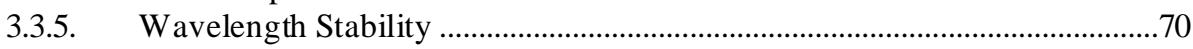

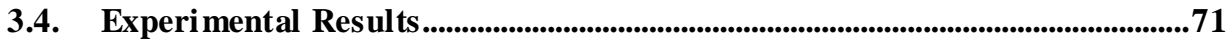

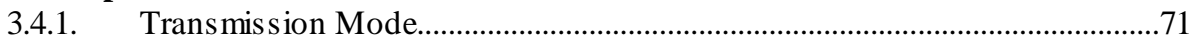

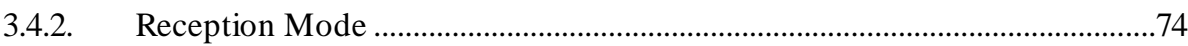

3.5. On the Capability of Multibeam Operation ...........................................................

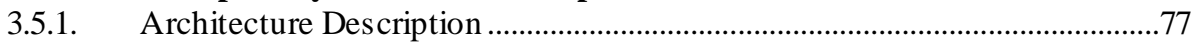

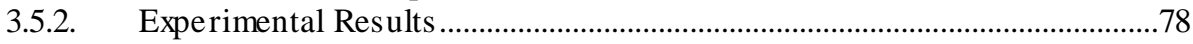

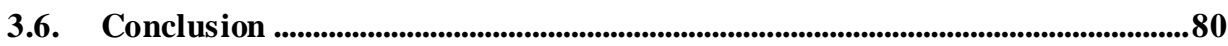

4. DIRECTION OF ARRIVAL ESTIMATION OF BROADBAND SIGNALS IN PHASED ARRAYS BY PHOTONIC

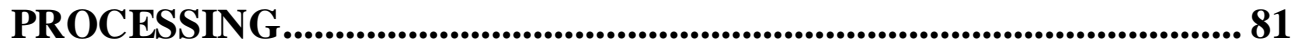




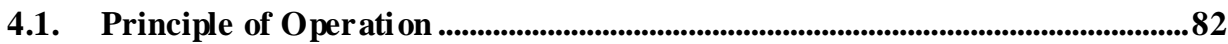

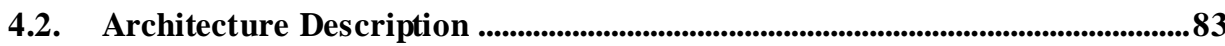

4.3. Experimental Results ............................................................................................................................86

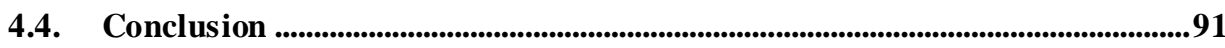

5. HETERODYNE OBFN BASED ON INTEGRATED BUTLER

MATRICES .................................................................................................. 92

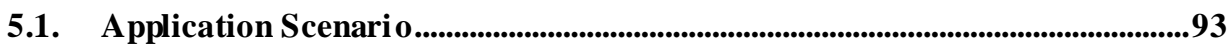

5.2. Optical Butler Matrices for Electrical Beamforming ..........................................96

5.2.1. Principle of Operation: Heterodyne Phase Shifting ……...............................97

5.3. 2N Beam Directions with a NxN Optical Butler Matrix .....................................99

5.4. Design of an Integrated Optical Butler Matrix ................................................100

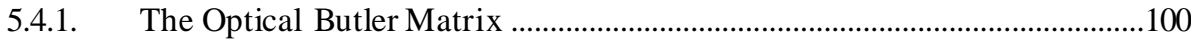

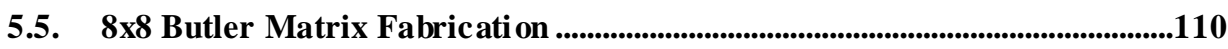

5.6. Characterization of the $8 \times 8$ B utler Matrix ..............................................................111

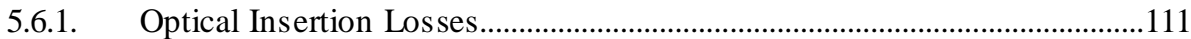

5.6.2. Phase Shift Performance of a 8x8 Butler Matrix .............................................112

5.7. Impact of experimental results on System Performance .....................................114

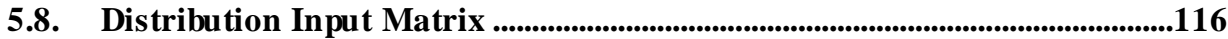

5.8.1. DIM structures and sample characterization ...............................................116

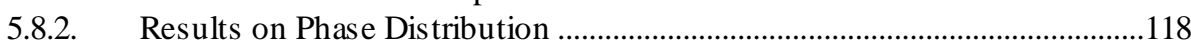

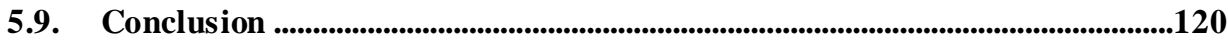

6. RF-LIKE OBFN BASED ON INTEGRATED OPTICAL BUTLER MATRICES ............................................................................. 121

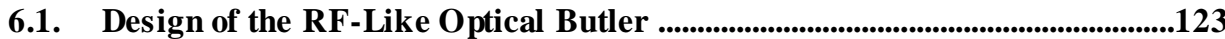

6.1.1. Basic Optical Coupler Equivalent to $2 \times 2$ Hybrid RF Coupler or $2 \times 2$ Butler Matrix 123

6.1.2. $4 \times 4$ Optical Butler Matrix

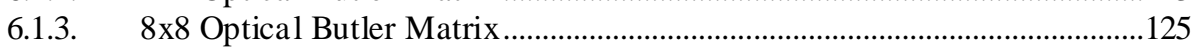

6.2. RF-Like 8x8 Optical B utler PIC manufacturing ............................................128 
6.3. RF-Like 8x8 Optical B utler PIC Eval uation ...............................................131

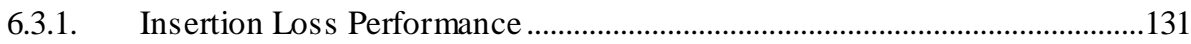

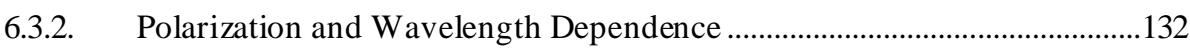

6.3.3. Phase Shifting Performance of the Matrix .......................................................133

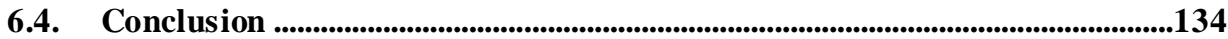

7. PHOTONIC MICROWAVE SIGNAL GENERATION....... 135

7.1. Introduction ..........................................................................................................................135

7.2. Photonic generation of wireless signals.................................................................................136

7.3. Fundamentals of Quadrature Modulations ..........................................................138

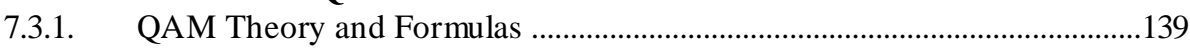

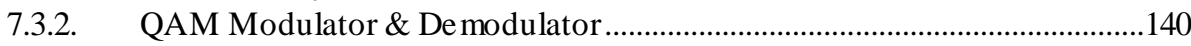

8. PHOTONIC GENERATION OF ELECTRICAL VECTOR MODULATIONS AT MICROWAVE/MILLIMETRE-WAVE

FREQUENCIES ................................................................................ 142

8.1. Photonic Vector QPS K Modulator Architecture ................................................142

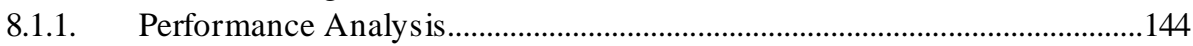

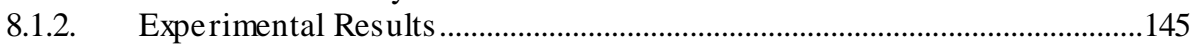

8.2. Photonic Vector QAM Modulator Architecture ..................................................148

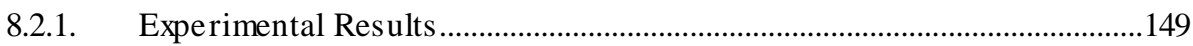

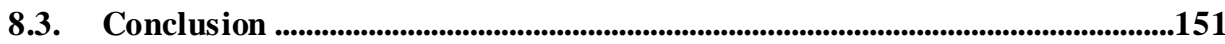

9. SIMULTANEOUS BASE-BAND AND MM-WAVE DELIVERY OF GB/S DATA EMPLOYING PHOTONIC VECTOR MODULATORS .............................................................................................. 152

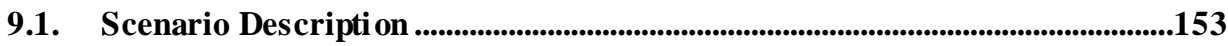

9.1.1. Application Scenario 1: Point-to-point Fixed Wireless Access (FWA) connections 153

9.1.2. Application Scenario 2: Coverage extension via wireless of

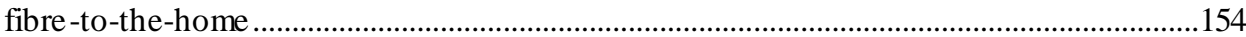

9.1.3. Application Scenario 3: Uncompressed HD video trans mis sion ..................155

9.2. Architecture Description and Experimental Setup ........................................156

9.3. Experimental Measurements .........................................................................................................157 
9.4. Photonic Integrated Circuit implementing a Heterodyne Photonic Vector Modulator

9.4.1.

Mathematical Model

9.4.2.

Discussion

\section{PHOTONIC VECTOR DEMODULATION}

\section{ARCHITECTURE FOR REMOTE DETECTION OF M-QAM} SIGNALS 163

10.1. Architecture Description ....................................................................................163

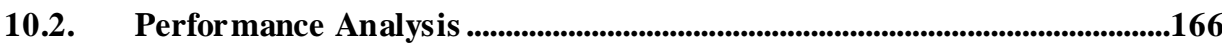

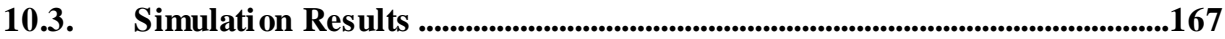

10.4. Experimental Results for QPS K modulations ..............................................169

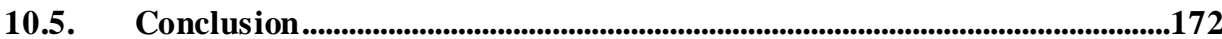

11. CONCLUSION ......................................................... 173

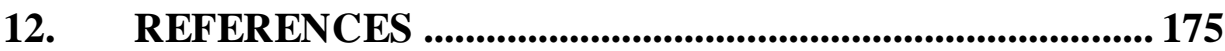

13. ANNEX A: IMPACT OF ELEMENT FAILURES IN AN OPTICAL BUTLER MATRIX .......................................................190

A.1 Basic for mulas for the effect of errors in large antenna arrays ......................190

14. ANNEX B: FABRICATION PROCESS IN SOI ................ 193

Spin Coating ...................................................................................................................................194

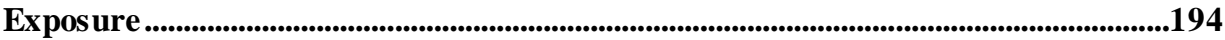

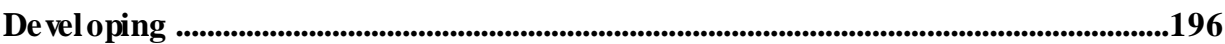

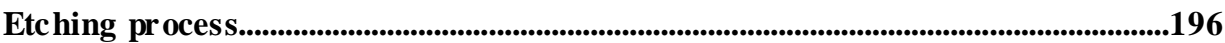

15. ANNEX C: ANALYTICAL MODEL OF THE PHOTONIC VECTOR MODULATOR.................................................................203

C.1. Simplified PVM model ..........................................................................................................203 
C.2. Noise analysis .208

C.3. Complete PVM model with a second-or der dis persive link (QB biasing).....210 C.4. Complete model with a second-or der dis persive link (MATB biasing) ..........215 C.5. Si mplified PVM model wi thout LO leakage .217

C.6. Complete 3-lasers PVM model including a second-order dispersive link (QB biasing) 


\section{List of Figures}

Figure 1. a) Diagram of an optical fibre consisting of: (1) glass core, (2) cladding, (3) buffer and (4) jacket; b) Light propagation in an optical fibre.........4

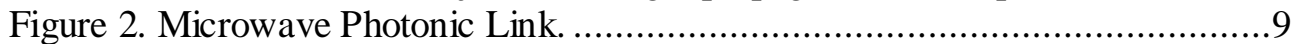

Figure 3. Optical Microwave processing system. ...........................................

Figure 4. Scheme of an externally modulated analog dispersive optical link [COR01].

Figure 5. Side view of simplified DFB (a) and DBR (b) laser structures. ............12

Figure 6. P-I curves at several temperatures (a) and RIN spectra at several power levels (b) of a typical 1550-nm semiconductor laser [AGR93]............13

Figure 7. Schematic of the Mach-Zehnder modulator transfer function. ............... 15

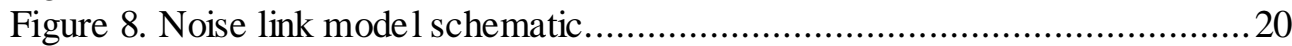

Figure 9. Optical frequency conversion of an IF modulated optical signal with an optical MZM modulator in a dispersive link [COR01].....................23

Figure 10. Electrical spectrum of a microwave photonic frequency converter at the output of the photodiode. Left picture shows a simulation and righ one is

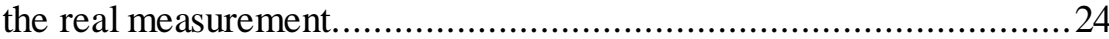

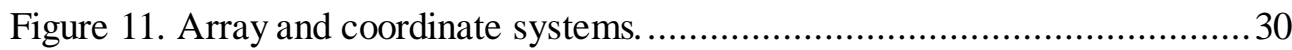

Figure 12. Wideband effects in phased array performance: (a) beam squint for a phased array (wavefronts and beam peak motion); (b) array 3-dB bandwidth versus $(L / \lambda) \sin \left(\theta_{0}\right)$ ( $B b$ is beam broadening factor) [KIN72].

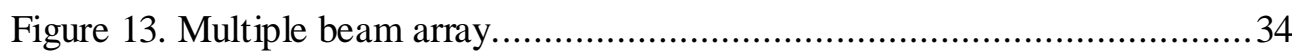

Figure 14. Eight-element, eight-beam Butler matrix and radiated beams..............35

Figure 15. Microwave Butler Matrixes. (left) 3D Orthogonal double set of 8x8 Butler matrixes and (right) example of a $8 \times 8 \mathrm{RF}$ matrix at $30 \mathrm{GHz}\left(15 \times 8 \mathrm{~cm}^{2}\right)$.

Figure 16. Rotman lens beamforming network ........................................... 37

Figure 17. Wideband array control: (a) array with TDUs; and (b) array with cascaded

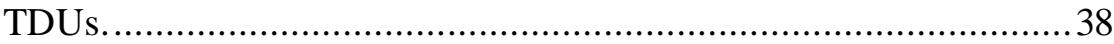

Figure 18. Architectures for fractional bandwidth wideband arrays: (a) phased array with contiguous time-delayed subarrays; and (b) phased array with timedelayed overlapped subarrays........................................................... 38

Figure 19. Free-space, 3D TTD beamformer based on SLM matrix [DOL95]. .....43

Figure 20. Free space TTD design based on MEMS mirrors. ............................44

Figure 21. Binary programmable fibre-optic delay line architecture [SOR84].....44

Figure 22. The binary fibre-optic delay line (BIFODEL) architecture based on switched single mode fibres.

Figure 23. Passive optical TTD architecture for receive muti-beam antenna, based on fibre fibre-optic delay lines [ALA95]......................................46 
Figure 24. Block diagram of the optical delay line architecture based on fibre dispersion [SOR92].

Figure 25. 2-D mode beamformer for transmit array antenna based on Esman's dispersion fibre prism. ........................................................... 47

Figure 26. FBG-based photonic beamformer architecture.................................48

Figure 27. FBG-based, TTD beamformer proposed in [LIU02] ..........................49

Figure 28. Optical beamformer based on a wavelength-division demultiplexer in transmit conf iguration [RAZ05].

Figure 29. Beamformer based on the spectral periodicity of an arrayed waveguide grating and dispersive fibre.......................................................5 50

Figure 30. Integrated, $1 \times 8$ optical TTD device based of 12 optical ring resonators [ZHU06].

Figure 31. Generic heterodyne optical beamformer architecture implemented with optical fibres.

Figure 32. Schematic diagram of an optical beamformer with TTD and phase and amplitude control [VID06].

Figure 33. Integrated, 16-channel InP beamformer chip [VLI99].....................55

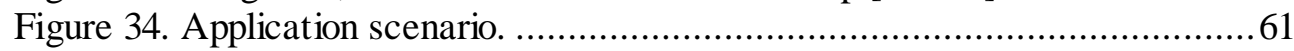

Figure 35. Photonic beam switched architecture for the transmission mode. ........62

Figure 36. Photonic beam switched architecture for the reception mode. .............63

Figure 37. Photonic 3-bit beamformer in receiving mode combined with the control electronics and the array antenna. PC: Personal Computer used to control the beamformer. PCE: Photonic Beamformer Control Equipment (bias source used to bias several components).

Figure 38. Antenna pattern used for calibration of antenna elements 3-4. (Solid) theory, (dotted) measured.

Figure 39. CNR estimation as a function of the laser source RIN for several system electrical bandwidths (left) and of the system electrical bandwidth (BW).

Figure 40. CNR estimation as a function of the noise figure of both EDFAs (left) and Noise power of the different noise terms as a function of the system electrical bandwidth (right).

Figure 41. CNR estimation as a function of $m_{R F}$ for different system electrical bandwidths (left) and Detected RF power (fundamental harmonic) vs. optical modulation index, $m_{R F}$ (right).

Figure 42. RF detected power versus RF input power showing the $1 \mathrm{~dB}$-compression point (left) and $1 \mathrm{~dB}$-compression point versus $\mathrm{V}_{\pi}$ (right)................. 70

Figure 43. Experimental setup for the transmission mode. .................................72

Figure 44. Schematic (left) and photograph (right) of the measurements set-up used to obtain radiation patterns. PSB: Photonic Switched Beamformer. .... 73

Figure 45. Measured radiation pattern at $40 \mathrm{GHz}$ for the 8 beam positions in transmission. Inset: (Dashed) Measured radiation pattern corresponding 
to the switching state $0000(340 \mathrm{~m})$, (solid) theoretical radiation pattern.

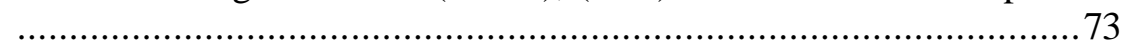

Figure 46. Constellation of the $155 \mathrm{Mb} / \mathrm{s} 32$-QAM after detection (Tx mode)......74

Figure 47. Experimental setup for the reception mode. ................................ 75

Figure 48. Measured antenna radiation pattern over the 90 degrees sector in receiving

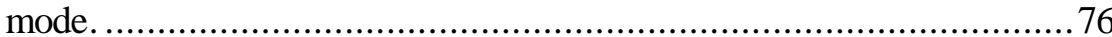

Figure 49. Constellation of the $155 \mathrm{Mb} / \mathrm{s} 32-\mathrm{QAM}$ after detection (Rx mode)......76

Figure 50. Block diagram of the multibeam beamforming network based on notch

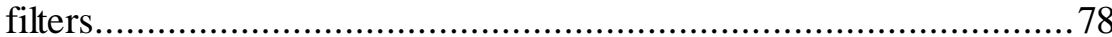

Figure 51. Experimental setup used to characterize the multibeam optical delay line

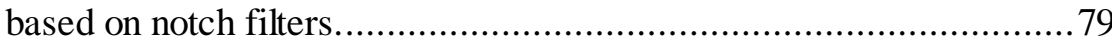

Figure 52. Time delay measurements for beam\#1 (length: $0 \mathrm{~m}$ ) and beam\#2 (length:

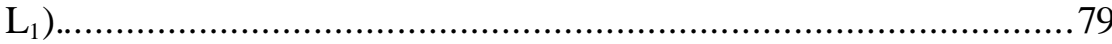

Figure 53. Time delay measurements for beam \#1 (length: $\mathrm{L}_{1}$ ) and beam \#2 (length:

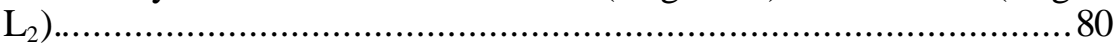

Figure 54. Incidence of a wavefront on a linear equally spaced antenna array. .....82

Figure 55. General model of an optical beamformer in reception mode. MZM: MachZehnder Modulator, TTD: True time-delay....................................83

Figure 56. DOA detection using a photonic microwave filter in an optical beamformer. ODL: Optical delay line ............................................. 84

Figure 57. Concept of DOA measurement using a photonic transversal microwave filter. The solid line represents the spectrum of a broadband signal, the dotted line the transversal filter response generated when the signals from two antennas are combined and the light dotted line the filter response when the emitter has changed its position. .................................... 85

Figure 58. Experimental setup used to show the feasibility of the proposed technique (LO: Local oscillator, att: attenuator, amp: amplifier)......................86

Figure 59. Spectrum of a $1 \mathrm{GHz}-3 \mathrm{~dB}$ bandwidth signal centred at $10 \mathrm{GHz} \ldots \ldots . . .87$ Figure 60. Broadband signal (1 GHz-3 dB bandwidth) with three notches (solid) and the original unfiltered signal (dotted).......................................... 88

Figure 61. Broadband signal ( $1 \mathrm{GHz}-3 \mathrm{~dB}$ bandwidth) with a single notch and the original unfiltered signal (dotted).

Figure 62. Measurement of the relation between the offset of the notch and the relative time delay between antenna elements/angle of arrival for the

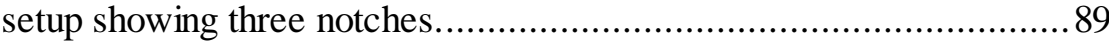

Figure 63. Measurement of the relation between the offset of the notch and the relative time delay between antenna elements/angle of arrival for the

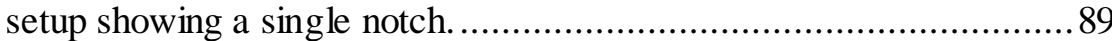

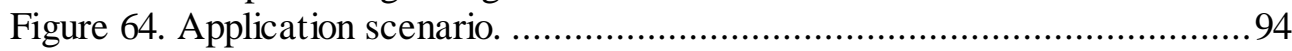

Figure 65. Beamforming network for the feeding of 256 elements by using $8 \times 16$ extended Optical Butler Matrices (up). 8x16 matrix employed in the beamforming network consisting in an extension of the $8 \times 8$ Optical 
Butler Matrix employing a scheme for the extension of the number of beams (down).

Figure 66. Optical Beamforming Network(OBFN) using a 2x2 optical Butler matrix.

Figure 67. Schematic view of the signal spectrum (both electrical and optical) at each of the indicated points in Figure 66.

Figure 68. Scheme of the $2 \mathrm{~N}$ extension for the OBFN...................................99

Figure 69. Optical Beamforming (OBF) using a 8x8 optical Butler matrix and Traditional $+2 \mathrm{~N}$ architecture.

Figure 70. Schematic view of the Butler Matrix (a) the Building Blocks' expected optical attenuation (b).

Figure 71. SEM images of two different waveguides. It can be seen the reduction of the sidewall roughness.... 101

Figure 72. SEM images of the reference waveguide structures and the waveguide with bends structures and a detailed view of the bend structures with different radii.

Figure 73. SEM image of a waveguides cross with an angle of 90 degrees and SEM images of the crossing point at the different angles considered. ........ 103

Figure 74. Experimental and 2D and 3D-FDTD simulated crosstalk losses as a function of the crossing angle.

Figure 75: SEM images of fabricated couplers (up). Detailed view of the waveguide separation in the middle of the coupler (down-left) and in the left extreme (down-right).

Figure 76. SEM image of a fabricated MZI structure with directional couplers. . 106

Figure 77. Experimental and simulated results of the transmitted power as a function of the wavelength for the cases of (a) reference structure, (b) 22.5 degrees, (c) 45 degrees and (d) 67.5 degrees.

Figure 78. SEM and optical microscope images of the fabricated 8x8 Butler matrix. 110

Figure 79. Experimental and simulated results of the normalized transmitted power as a function of the wavelength. The title of each plot describes the input port.

Figure 80. Initial DIM structure of $2 \mathrm{~N}$ architecture (left) and alternative one (rigth) with better symmetry.

Figure 81. Optical microscope image of a made DIM+Matrix sample. ............ 118

Figure 82. Structure of the DIM+Matrix chip................................................ 118

Figure 83 . RF-like $2 \times 2$ hybrid coupler scheme by using optical couplers and delay lines (left), the corresponding unitary RF transfer matrix $\mathrm{T}$ (right-up) and the demonstration of the orthogonality of $\mathrm{T}$ (right-down)................ 123

Figure 84. RF-like 4x4 Butler matrix (top) and its corresponding unitary RF transfer matrix T4x4 (bottom). ............................................................ 124

Figure 85. Simplified RF-like 4x4 Butler matrix with less $3 \mathrm{~dB}$ couplers........... 125 
Figure 86. Scheme of an optical Butler matrix of 8x8 ports implemented by optical splitters/combiners and optical delays........................................ 126

Figure 87. Transfer matrix [T] for the 8x8 matrix drawn in Figure 86.............. 127

Figure 88. 64x64 Optical Beamforming Network based on 8x8 RF-like OBMs. FGU: Frequency generation Unit; EOM: Electro-optical modulators ........ 128

Figure 89. (a) SEM of a low contrast silica-based optical waveguide and (b) mode profile for a low contrast silica-based optical waveguide with a width and thickness of $6 \mu \mathrm{m}$. The extremely low roughness of the waveguide wall suggest that propagation losses lower than $0.09 \mathrm{~dB} / \mathrm{cm}$ can be achieved once it will be covered with un-doped silica................................. 129

Figure 90. Microscope image of the MMI structures.................................... 129

Figure 91. MMI response in a $50 \mathrm{~nm}$ wavelength. MMI with $26.5 \mu \mathrm{m}$ width and

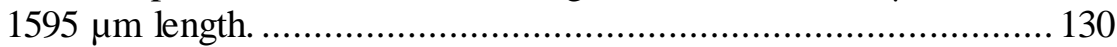

Figure 92. Layout of the designed 8x8 RF-like Optical Butler Matrix ............... 130

Figure 93. 8x8 RF-like Optical Butler Matrix Packaged and pigtailed. ............. 131

Figure 94. Optical Power vs wavelength for TE polarization.......................... 132

Figure 95. Optical Power vs wavelength for TM polarization........................... 132

Figure 96. Rectangular representation of the polar diagram (left) and Bit sequence mapping for a 16QAM signal.................................................. 138

Figure 97. Basic QAM modulator (left) and demodulator (right) diagram. ......... 141

Figure 98. Photonic vector modulator (PVM) architecture............................. 142

Figure 99. Optical spectrum at the photodiode input.................................... 146

Figure 100. Constellation and quadrature-phase eye diagram for the generated QPSK signal.

Figure 101. Electrical spectrum of the generated signal at $37.4 \mathrm{GHz}$. Inset: Electrical spectrum at photodiode output from 0 to $40 \mathrm{GHz}$.

Figure 102. Multilevel Photonic Quadrature-Amplitude-Modulator Architecture.

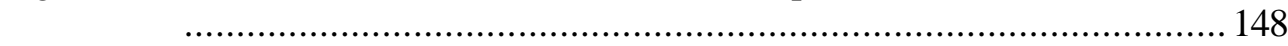

Figure 103. Electrical spectrum at the photodiode output............................. 150

Figure 104. Top: Demodulated eye diagrams of the In-phase (left) and Quadrature (right) components. Bottom: Intermediate eye (left) and the generated 8QAM constellation diagram (right)........................................ 150

Figure 105. High-speed point-to-point FWA Application Scenario. (upper) A realtime wireless backup link. (lower) Point-to-point FWA connection. . 154

Figure 106. Fibre-to-the-air application scenario ........................................ 155

Figure 107. Uncompressed HD video signal transmiss ion application scenario. . 155

Figure 108. Schematic of the proposed PVM architecture and phase-matched demodulation arrangement employed in the experiments. ................ 156

Figure 109. Measured electrical spectrum at PD output (left) and detected $2.5 \mathrm{~Gb} / \mathrm{s}$ 4-ASK signal down-converted from $39.23 \mathrm{GHz}$ (right)................. 157

Figure 110. Measured eye-diagrams of $1 \mathrm{~Gb} / \mathrm{s}$ parallel bit streams after demultiplexing measured by the DCA: $\mathrm{b}_{0}{ }^{(\mathrm{I})} @ 1550.12 \mathrm{~nm}(\mathrm{left}), \mathrm{b}_{0}{ }^{(\mathrm{Q})} @$ 
$1549.32 \mathrm{~nm}$ (mid), $\mathrm{b}_{1}{ }^{(\mathrm{I})} @ 1548.52 \mathrm{~nm}$ (right). Horizontal scale is $200 \mathrm{ps} /$

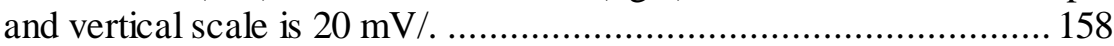

Figure 111. Schematic of the mmW transmitter. ........................................... 160

Figure 112. Schematic of the receiver for demodulation of the wireless signals. 162

Figure 113. Photonic vector demodulator architecture................................... 164

Figure 114. Photonic demodulation process concept. .................................... 165

Figure 115. VPI simulation block diagram. Simulated electrical spectrum at photonic QAM transmitter output. Received 64QAM in-phase component eye diagrams using standard electrical demodulation (left) and the proposed photonic QAM receiver (right)................................................. 168

Figure 116. Radiofrequency QPSK up-converted signal spectrum at $42.7 \mathrm{GHz}$. 170

Figure 117. Optical spectra at the photodiodes input. The solid line represents the inphase amplitude-modulated optical carrier and the dashed line corresponds with the quadrature amplitude-modulated optical carrier.

Figure 118. Demodulated constellation diagram.............................................. 171

Figure 119. Received in-phase (left) and quadrature (right) spectra................. 171

Figure B.1. SEM image of a SOI waveguide. It can be clearly seen the top silicon layer, the oxide cladding layer (BOX layer) and the silicon substrate.

Figure B.2. Schematic of the fabrication processes. A fabricated Mach-Zehnder Interferometer using the lift-off process, which includes two optical couplers and two phase shifters, is depicted in the inset................... 194

Figure B.3. Exposure patterns for (a) resist mask or (b) lift-off process.............. 195

Figure B.4. Lay-out for building block consisting on different angles for waveguide crosses

Figure B.5. Improved sidewall roughness by modifying e-beam lithography process and tuning the bias during the etch step............................................... 196

Figure B.6. Improved results after exposure dose adjustments.......................... 196

Figure B.7. Guide roughness comparison between resist mask etching (a) and lift-

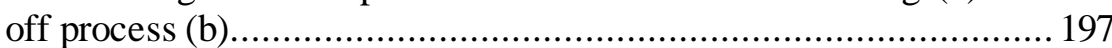

Figure B.8. Schematic of the fabrication process.......................................... 198

Figure B.9. Scanning electronic microscope (SEM) image of a coupler defined (a) with a standard process and (b) including proximity effect compensation.

Figure B.10. Cases to analyze the influence of waveguide variations on the phase error.

Figure B.11. Three times the standard deviation of the phase error as a function of the length of the sections, $\Delta L$, and the standard deviation of the sidewall variations, $\sigma_{s d}$ for the two cases shown in Figure B.10. The total length, $L$, is $26 \mu \mathrm{m}$. 200 
Figure B.12. Normalized autocorrelation of the waveguide width variations measured with a an automatic measurement process performed by a SEM (top) and with a image post-processing (bottom). .......................... 202

Figure C.1. Detected LO/Data power ratio $(\mathrm{dB})$ as a function of laser $\mathrm{P}_{\mathrm{ON}} / \mathrm{P}_{\mathrm{OFF}}$ ratio.

Figure C.2. PVM noise contributions as a function of received optical power. ... 209 Figure C.3. Dependence of the Factor ${ }_{\mathrm{LO}}$ on the modulation index.....................210 Figure C.4. Block diagram a PVM with a third laser to avoid LO leakage.......... 217 


\section{List of Tables}

Table 1: Typical parameters of the Standard Single-Mode Fibre (SSMF)..............6

Table 2: A tentative classification of concepts and techniques for optical beamforming.......................................................................... 41

Table 3: Comparison Of Main Optical Beamforming Concepts And Technologies

.

Table 4: Required fibre lengths as a function of the wavelength separation and fibre type ...................................................................................64

Table 5: Correspondence among switching voltages, overall delay and steering angle with respect to broadside. (Switch control '0' corresponds to cross state and ' 1 ' to bar state)

Table 6: System parameters values used in the performance estimation simulations.

Table 7: Simulation results of the effect of wavelength excursion on achieved delays.

Table 8: Residual secondary lobes relative to the main lobe............................... 71

Table 9: Variation on the directivity for several directions. ..............................71

Table 10: Time delay measurements and the expected value............................90

Table 11: Statistical analysis of the measurements. Better results are obtained for lower doses which exhibits lower proximity effects. ........................ 105

Table 12: Summary of the fabricated Building Blocks and Effect in the Radiation

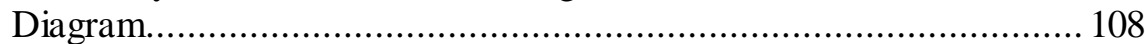

Table 13: Measured output power in $\mathrm{dbm}$ as a function of the input port. The last columns show the total power in the eight output ports and the standard deviation of the output power variation. .......................................... 111

Table 14: Comparison between the ideal and measured phase difference and the obtained statistical deviation of the phase shift error when light is injected through each one of the input ports. .............................................. 114

Table 15: Error Budget Calculations for a Complete BFN including 8x8 BM Test-

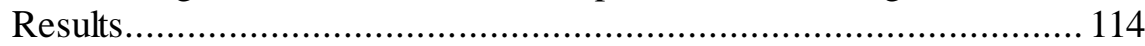

Table 16: Summary of Errors and their effect on the antenna radiation pattern ... 115

Table 15: Phase Performance of the DIM+Matrix structure (Average of

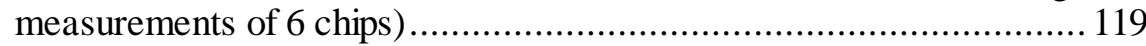

Table 16: Optical Insertion loss of the packaged OBM chip............................. 131

Table 17: Relative phases to the OUT1 of the Matrix @ 19.95GHz................... 133

Table 18: System tolerances and LO tuning bandwidth for different LO frequencies considering a maximum quadrature phase error of 1 degree. Fibre dispersion parameter for depicted values is $D=17 \mathrm{ps} / \mathrm{km} \cdot \mathrm{nm}$............ 145

Table 19: System Tolerances vs. LO frequency.............................................. 166

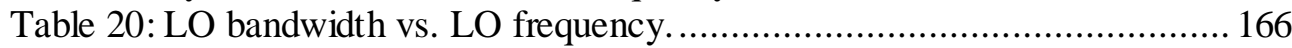




\section{CHAPTER 1}

\section{Introduction to Photonic Technology}

The process of harnessing light to perform a wide range of functions has been one of the main breakthroughs in recent history ${ }^{1}$. Light is at the core of a wide range of technologies from long-haul data transmission networks which make Internet and modern communications possible to illumination, industrial cutting, medical technology and life sciences, just to cite a few. It is expected that light-related technologies will reach a worldwide market of 650 million Euros in 2020 [www1]

The development of the laser in the early 60s [MAI60] and the proposal of optical fibre [KAO66] as a transmission medium for long distances by Dr. Charles K. Kao, who was awarded the Nobel Prize in Physics in 2009 by this achievement, jointly with the subsequent technological improvements, revolutionized our way of life and led to the development of a new scientific field: photonics. Photonics is the technology of generating, controlling, and detecting light waves and photons and their application in a wide range of fields.

There are three main platforms for controlling the propagation of photons. One is based on its propagation on air (without a guided medium) while the other two rely on dielectric optical waveguides. These three are:

\footnotetext{
${ }^{1}$ As shown, for example, by UNESCO recognition of 2015 as the International Year of Light
} 
1) Free space bulk optics relies on components as lenses, mirrors, prisms, spatial light modulators, etc. This was the first method to be developed and has been very successful in many applications since the propagation medium is air, which is mainly non-dispersive, and this approach offers a high degree of parallelism. However, for communications, free space optics do not allow the transmission of signals over long distances in terrestrial applications and results in rather bulky implementations that are prone to misalignment and difficult to integrate. A couple of exceptions are the systems for free-space laser links for short range communication backhauling, used normally as back-up options due to its low performance under rain and fog conditions, and its version for inter-satellite communications, which is gaining momentum since it enables high-speed communications between in-orbit satellites with minimum latency in long distances thanks to the absence of atmosphere and its associated attenuation.

2) Fibre optics. The development of low-loss flexible dielectric waveguides based on total internal reflection for the transmission of high speed signals was a key breakthrough that allowed the present Information Age. From its original inception as a transmission medium for long-haul communications, optical fibre expanded to many other fields and different kinds of speciality fibre were developed to fill niche applications.

3) Integrated Photonic Circuits (PIC) are based on integrating on a single chip a set of optical components and pigtailing the PIC to an optical fibre to implement a set of functions directly in the optical domain. It is a parallel evolution to the development of integrated electronic circuits from the original electronic systems made of discrete components. PICs are being used commercially in core and metro digital communication networks as well as in data-centres ${ }^{2}$ and are being also investigated for analogue applications and RF signal processing.

\subsection{Fibre-optic Systems}

Fibre-optics is the technology based on optical fibre cables. The optical fibre is a dielectric and extremely thin transmission medium made of silica (drawing glass) or plastic. Optical fibre is an outstanding transmission medium which revolutionized data communications due to its unique combination of low loss and wide bandwidth.

2 See http://www.infinera.com/ for InP PICs or http://www.luxtera.com/ for Silicon Photonics PICs. 
Unlike coaxial or copper wires, optical fibres allow the transmission over long distances with extremely wide bandwidths. Fibres are also used because signals travel through them with lower loss per $\mathrm{km}$ than in metal wires, and they are unaffected by electromagnetic interference.

Due to these features, optical fibres have been massively deployed. Present telecommunication networks rely heavily on them. Initially, the low loss and high bandwidth provided by optical fibre were exploited to implement long-haul submarine and continental links interconnecting telecom operators. Later, their use was expanded to interconnect central stations and regional networks and, nowadays, optical fibre make the core of carrier networks and has reached the last mile with strong penetration in access networks in the form of Fibre to the Home (FTTH) services. Additionally, fibres can be used as sensors to measure strain, temperature, pressure, and other qualities by modifying a fibre so that the property to measure modulates the intensity, phase, polarization, wavelength, or transit time of light in the fibre. Fibre optics sensing is most commonly found in security systems. By placing optical fibres along a boundary such as a fence or property line, an optical signal can be used to monitor for disturbances and will trigger an alarm if an intrusion occurs. Another field were optical fibre is a key element is in fibre lasers. Dopedfibres can be used to implement a wide range of optical sources in a range of wavelengths. This field includes femtosecond pulsed lasers for basic science and spectroscopy to high power sources used for industrial cutting and material processing. Finally, other niche applications include transmission of power using a photovoltaic cell to convert light into electricity, fibre optical gyroscopes, artistic implementations, etc.

The first glass fibres were fabricated almost a century ago (in the 20's of the XX century), although their applicability was very limited up to the 50's when the addition of a cladding led to an important improvement of their guiding capabilities [AGR10]. However, originally, their losses $(1000 \mathrm{~dB} / \mathrm{km})$ made them impractical for communication applications. In the $70 \mathrm{~s}$, dramatic improvements on the fabrication led to losses below $20 \mathrm{~dB} / \mathrm{km}$ and by 1979 the now common value of 0.2 $\mathrm{dB} / \mathrm{km}$ at $1550 \mathrm{~nm}$ was reached. The availability of optical waveguides with such small losses revolutionized the field of optical fibre communications in the following decades.

An optical fibre is basically a cylinder made of silica glass which has been covered by a material with lower refractive index. Because of the principle of total internal refraction [AGR10] the light is guided through the cable with little loss. Figure 1.1a shows the structure of a common fibre where a core made of silica is covered by a cladding and a jacket, both with lower refractive indexes to confine light. According to the radial variation of the refractive index of the core they can be 
classified as step-index or graded-index fibres. For the first type there is no variation while for the second one the index decreases for increasing radius, usually following a parabolic curve. The guiding properties of optical fibres can be observed in a first approximation by means of a ray picture such as the one shown in Fig. 1.1b. A ray incident in the centre of the core with a certain angle changes its direction of propagation according to Snell law:

$$
n_{0} \sin \left(\theta_{i}\right)=n_{1} \sin \left(\theta_{r}\right)
$$

The ray bends toward the axial axis and, considering the step-index fibre scenario, it is guided through the fibre as long as the angle of incidence with respect to the core-cladding interface is large enough to produce total internal reflection.

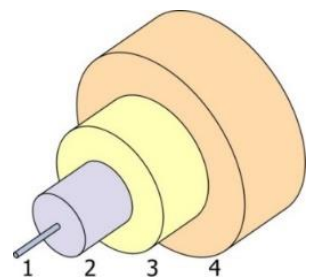

(a)

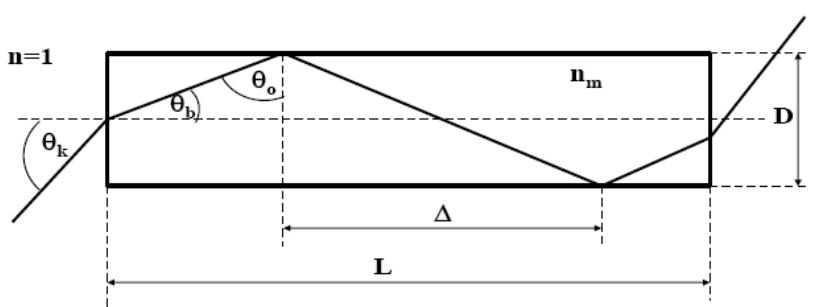

(b)

Figure 1. a) Diagram of an optical fibre consisting of: (1) glass core, (2) cladding, (3) buffer and (4) jacket; b) Light propagation in an optical fibre.

Although single-mode fibres avoid intermodal dispersion, group velocity is usually frequency dependent. Thus, propagating pulses get widened because its spectral components travel at different group velocities. This effect is known as group-velocity dispersion or chromatic dispersion.

Group-velocity dispersion (GVD) originates from two different sources, material dispersion and waveguide dispersion. The former arises from the dependence of the refractive index with the optical frequency while the latter depends on the physical characteristics of the fibre: radius and index difference. The dimensions of the fibre are usually tailored for the total dispersion to take certain values. Thus, it is possible to shift the dispersion in such a way that the zero-wavelength dispersion falls into the range of interest (dispersion shifted fibre or DSF) or to obtain dispersion which is opposite to that of standard fibre for compensation purposes (dispersion compensating fibre or DCF).

It is convenient to model the effect of dispersion by expanding the modepropagation constant around a given optical frequency $\omega_{0}$ [AGR97], 


$$
\beta(\omega)=n(\omega) \frac{\omega}{c}=\beta_{0}+\beta_{1}\left(\omega-\omega_{0}\right)+\frac{1}{2} \beta_{2}\left(\omega-\omega_{0}\right)^{2}+\frac{1}{6} \beta_{3}\left(\omega-\omega_{0}\right)^{3}+\ldots
$$

being $\beta_{n}$ the $\mathrm{n}^{\mathrm{th}}$-derivative of $\beta$ with respect to the optical frequency, $\omega$. More concretely, the definition of $\beta_{n}$ is:

$$
\begin{gathered}
\beta_{1}=\frac{1}{v_{g}} \\
\beta_{2}=-\frac{\lambda^{2}}{2 \pi c} D \\
\beta_{3}=\left(\frac{\lambda^{2}}{2 \pi c}\right)^{2}\left(D+\frac{\lambda}{2} S\right)
\end{gathered}
$$

where $c$ is the speed of light, $\lambda$ is the optical wavelength, $D$ is the dispersion parameter and $S$ is the dispersion slope. $\beta_{0}$ is the propagation constant at the central frequency, $\beta_{l}$ is for the propagation of the pulse envelope at the group velocity $v_{g}$ and higher order terms represent dispersion the group velocity dispersion of order two and three.

Although chromatic dispersion is, jointly with loss and nonlinearity, one of the main limitations in the achievable bitrate in any optical link, we can benefit from it and be exploited to implement different functions. An important use of dispersion will be described in the following section in this thesis as a tool for generating differential delays in optical signals at different wavelengths travelling in a dispersive media like the optical fibre. In particular, considering only the optical dispersion of second order, the differential delay $\Delta \tau[p s]$ accumulated between two optical signals with a wavelength separation of $\Delta \lambda[\mathrm{nm}]$ after the propagation through $L$ kilometres of optical fibre with a given dispersion $D[\mathrm{ps} /(\mathrm{km} \cdot \mathrm{nm})]$ can be calculated as:

$$
\Delta \tau=D \cdot L \cdot \Delta \lambda
$$

This differential delay between different spectralcomponents of an optical signal is also the origin of the well-known phenomenon of carrier suppression in a microwave photonic link when dual side band optical modulation is used [AGR10].

Typical parameters of the Standard Single-Mode Fibre (SSMF) are shown in Table 1[AGR10]. 
Table 1: Typical Parameters of The Standard Single-Mode Fibre (SSMF)

\begin{tabular}{|l|l|l|}
\hline Symbol & Parame ter & Value \\
\hline$\lambda$ & Working wavelength & $\begin{array}{l}1550 \mathrm{~nm} \\
\text { (most typical in communications and in MWP since the } \\
\text { better efficiency of optical amplification at this wavelength) }\end{array}$ \\
\hline$\alpha$ & Attenuation coefficient & $0,2 \mathrm{~dB} / \mathrm{km}$ \\
\hline $\mathrm{D}$ & First order dispersion & $17 \mathrm{ps} /(\mathrm{km} \cdot \mathrm{nm})$ \\
\hline $\mathrm{S}$ & Dispersion slope & $0,07 \mathrm{ps} /\left(\mathrm{km}{ }^{2} \cdot \mathrm{nm}\right)$ \\
\hline
\end{tabular}

\subsection{Photonic Integrated Circuits (PIC)}

SSMF is an amazing transmission medium due to its low loss and high capacity and has no competition for long and medium range data transmission. However, to manipulate optical signals additional components are needed as modulators, optical delay lines, filters, etc. Traditionally these components were implemented as discrete devices. This is no big issue in carrier grade links but for other applications targeting low-cost scenarios such as FTTH or in applications were many components are needed, the cost of the photonic solution may severely reduce its commercial feasibility.

In order to address this issue optical integration has been pursued as a way to introduce dramatic reductions in the cost and size of photonic subsystems [HUN09], [LOC11], [THY14]. It is expected that photonics will follow a similar evolution to electronics, where integration led to massive cost reductions, continuous improvements in performance and to a strong widening of its fields of application.

Several major technologies have evolved in the last decades for photonic integration: III-V compound semiconductors (GaAs, InP); nonlinear crystals (LiNbO3); dielectrics (silica and silicon nitride based waveguides) and element semiconductor (silicon-on-insulator, SOI). Each technology has its specific strength such as capability for light generation and detection, modulation, passive routing with low propagation loss, electronic integration, ease in packaging, etc. Nevertheless, monolithic integration without losing key functionalities has not yet been achieved.

Probably, the main platforms for photonic integration are: silica planar lightwave circuits (PLCs), InP and silicon-on-insulator (SOI). The propagation losses are similar in the last two technologies with the state of the art being around $1-2 \mathrm{~dB} / \mathrm{cm}$ : in in InP can be around $1.4 \mathrm{~dB} / \mathrm{cm}$ [STU99] and in silicon for commercial processes 
today they are typically around the $2 \mathrm{~dB} / \mathrm{cm}$ range [www2]. On the other side, PLC waveguides can offer lower attenuation $<0.1 \mathrm{~dB} / \mathrm{cm}$ [BAU11].

- Silica glass planar lightwave circuits (PLCs) are widely used as key devices for wavelength division multiplexing (WDM) transmission and fibre-to-the-home (FTTH) networks because of their excellent optical properties and easiness for mass production. In such applications, the PLCs have been used for wavelength multi/demultiplexers, optical add/drop or cross-connect switches and programmable filters [HIM98]. The silica-based waveguides are very popular due to their very low propagation loss. The lowest propagation loss has been demonstrated using a phosphorus-doped silica on silicon waveguide with a propagation loss of $0.85 \mathrm{~dB} / \mathrm{m}$ [ADA94]. However, this lower propagation loss was demonstrated on a waveguide with a low refractive index contrast of $0.7 \%$. Such a low contrast is less attractive for photonic chip integration since it only allows large bending radius and hence, larger chip size. Other materials like silicon nitride are also used in PLC circuits since it enables shorter bending radius with relatively low losses in the order of $<0.1 \mathrm{~dB} / \mathrm{cm}$ [BAU11].

- Indium Phosphide (InP) inherently supports light generation, amplification, modulation, detection, variable attenuation, switching and passive functionalities. For this reason and the contrast index which allows large scale photonic integration [WIL15] as have been consistently demonstrated by Infinera [KIS11]. In this case the PICs are highly complex with components (lasers, modulators, arrayed waveguide gratings) counting more than 400 in a single chip. However, this technology work with smaller wafers and the required facilities are not aimed for mass production since it is not compatible with the common CMOS processes for microelectronics.

- $\quad$ Silicon photonics is one of the most exciting and fastest growing photonic technologies in recent years [HOC13]. The initial pull of this technology is its compatibility with the mature silicon IC manufacturing. Another motivation is the availability of high-quality silicon-oninsulator (SOI) planar waveguide circuits that offer strong optical confinement due to the high index contrast between silicon $(n=3.45)$ and $\mathrm{SiO}_{2}(\mathrm{n}=1.45)$. This opens up miniaturization and large scale integration of photonic devices. Moreover, it has also been shown that silicon has good material properties like high third-order optical nonlinearities which, together with the high optical confinement in the SOI waveguides, enable functionalities like modulation, wavelength conversion [LOC11]. But Silicon is not well-suited for electro-optic 
modulators and detectors in $1550 \mathrm{~nm}$ operating wavelengths and the development of a practical silic on laser is still lacking which are strong drawbacks that are constraining silicon photonics from becoming the mainstream approach for deployed photonic components.

\subsection{Microwave Photonics}

Although originally targeted for long-distance communications, fibre optic technology has been applied to a wide range of fields, among them access networks, data centres, sensing, fibre lasers, illumination, imaging, and so forth. Another important application for fibre optic technology is the interaction between optics and microwaves for applications such as radar, communications, warfare systems, and instrumentation. This area has become known as microwave photonics (MWP) [SEE02], [CAP07], [WIL08], [MAR09], [BER10], [VID12], [URI15]. This interdisciplinary field benefits from the complementary features that optical technology can provide to microwave signals. Motivation on MWP research is justified by the capability of optical devices to distribute signals over long distances and process huge spectral bandwidths. This area of photonics includes the photonic generation and distribution, processing and monitoring of microwave signals, as well as photonic-assisted analogue-to-digital conversion, to cite its main topics.

Features such as the low loss and wide bandwidth of fibre optic technology can be exploited to provide functions and capabilities to microwave systems that are very complex or even not implementable when carried out directly in the microwave domain. These advantageous characteristics are especially relevant when high frequency signals are considered given current limitations in the generation, processing, and distribution of microwave, millimetre- and submillimetre-wave signals.

The first MWP systems were employed for optical distribution of electrical signals as shown in Fig. 2. An electrooptical device (E/O), typically a Mach-Zehnder modulator but also electroabsorption modulators or direct modulation of an optical source are possible, transfers the electrical signal onto one, or more, optical carriers which are distributed through an optical medium (fibre) before being recovered in the electrical domain in an optoelectronic converter (O/E), typically a PIN photodiode [COX04]. Such a system is known as a photonic microwave link. Its main advantages come from the medium properties: low and constant attenuation over the entire microwave and millimetre-wave modulation frequency range, which allows the distribution of signals for hundreds of kilometres with small degradation; independence of data format, which means that baseband and radio frequency (RF) signals can be transmitted with equal performance; huge bandwidth; flexibility to 
adapt to different deployment scenarios due to the inherent flexibility of fibre cables; low weight and volume; and immunity to electromagnetic interferences. These features allowed the implementation of radio-over-fibre $(\mathrm{RoF})$ systems (also known as analogue links) which perform distribution of radio signals, usually allocated in the microwave and millimetre bands, from a central station (CS) to one or more base station (BS) [NOV98]. This approach offers the ability to reduce system complexity by using a centralized architecture that incorporates a simplified antenna module located closer to the customer. The electrical signals transmitted can be allocated in baseband with respect to the optical carrier, at an intermediate frequency (IF) or directly at the desired radiation band. The best option will depend on the number of BSs although the latter is commonly employed because it allows the use of simple BSs.

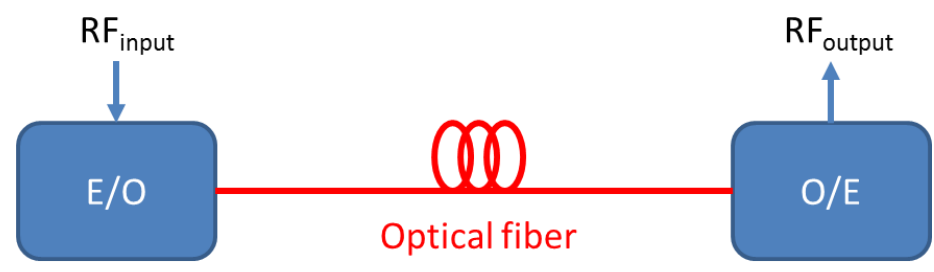

Figure 2. Microwave Photonic Link.

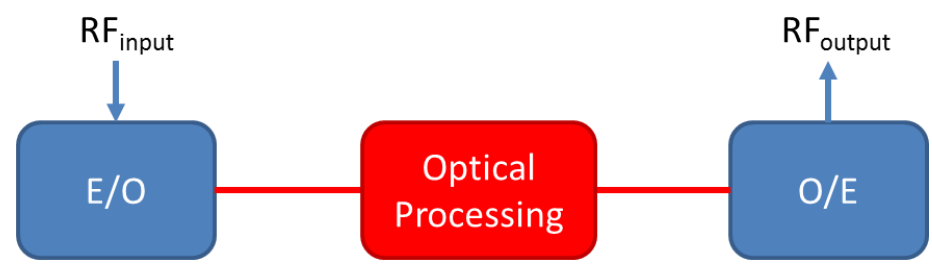

Figure 3. Optical Microwave processing system.

Besides microwave signal transmission, fibre optics can also be employed to process microwave and millimetre-wave signals directly in the optical domain. Optical microwave signal processing was first proposed in 1976 [WIL76]. Optical signal processing (Figure 3) offers the unique capability of controlling microwave signals with ultrawide bandwidth and at almost any frequency of the microwave and millimetre-wave spectrum breaking the bottlenecks of pure electronic devices. Besides, optical processing using optical waveguides allows the realization of large time $\mathrm{x}$ bandwidth products [ZMU94]. Therefore, optical signal processing (e.g. [TAY79], [JAC87], [TED95], [MIN06], [CAP13]) represents a new approach to the problem of signal processing that complements digital processing and direct processing using microwave components. Besides, processing microwave signals 
directly in the optical domain avoids costly optoelectronic conversions if the signals are already in the optical domain for distribution using optical fibre.

Optical signal processing applications include microwave filtering (e.g. [CAP05]), multigigabit per second analogue-to-digital (A/D) converters ([COP99], [JUO01], [HAN03]), frequency converters and mixers [ROU98], signal correlators [HUN99], arbitrary waveform generators [CHO03] and beamformers for phased arrays.

Despite these advantages and the wide range of applications where microwave photonic systems could be used, the potential of this technology to replace electronics by optical devices has been hampered by the high cost of microwave photonic systems what limits the practical use of radio-over-fibre distribution networks to very specific applications. The high cost is mainly due to the need of many different components to perform microwave functionalities, and each component being packaged individually. The need of many components also leads to quite bulky implementations with high power consumption.

These issues can be overcome through photonic integration [MAR12]. As happened in the microelectronic industry, many applications can be realized in a much more compact and cost-effective way by integrating the required functionality in a single chip. Among the different integration technologies proposed so far, Silicon Photonics (or CMOS photonics) stands out due to its compatibility with mature materials and processes of the microelectronic industry. That results in an easier way to bring photonic ICs (Integrated Circuits) to the market and to reduce the product cost. Additionally, since each single subsystem (laser, modulator, filter...) will have an insignificant cost, industry will concentrate on achieving the desired functionality using as many components as needed by enabling Large Scale Integration (LSI).

Photonic CMOS integration will result in the development of a small set of elementary building blocks suitable for handling complex analogue signals which will contribute to the deployment of very-high-bit rate and heterogeneous wired/wireless access networks.

\subsection{Microwave Photonic Links}

As described in the previous section, and shown in Figure 3, a basic microwave photonic system is composed by an optical transmitter that converts the electrical signal to the optical domain, a propagation medium, being normally optical fibre for the signal transportation (as in microwave photonic links) or optical waveguides 
implemented in PICs, and an opto-electronic converter, usually a PIN photodetector for typical microwave photonic applications.

The optical transmitter is composed by a continuous wave (CW) lasers that can be directly modulated through their bias current or externally modulated by an optical modulator, which exhibits a higher modulation bandwidth and is going to be the option chosen in this thesis. A more general scheme of a MWP link is show in the following figure according with the notation described in [COR01] for the case of external modulation and optical dispersive media.

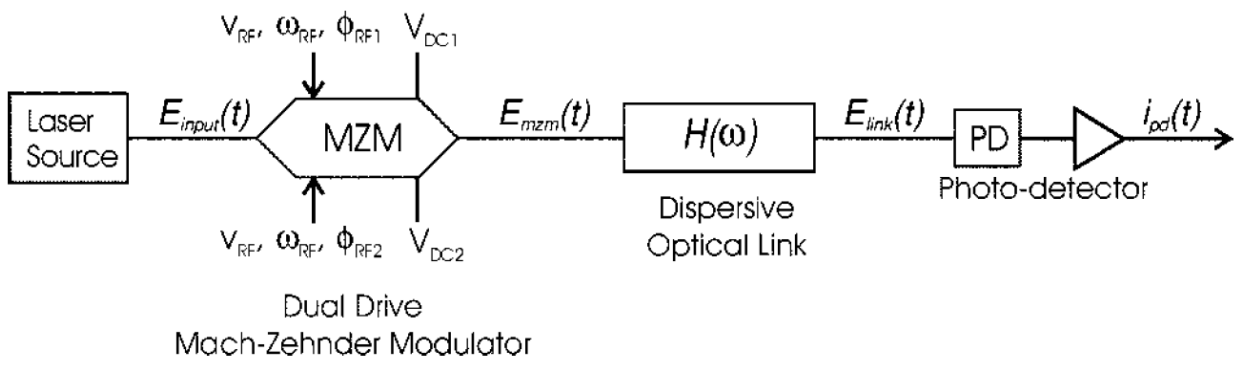

Figure 4. Sche me of an externally modulated analog dispe rsive optical link [COR01].

The CW lasers normally used in MWP systems are based on distributed feedback (DFB) lasers [AGR93], which consist in a cavity of semiconductor media of optical gain. The feedback in DFB lasers is not localized at the facets, but distributed throughout the cavity length. This is achieved through a periodic variation of the refraction index. Mode selectivity of the DFB results from the Bragg condition, which states that coupling of the waves propagating in the forward and backward directions occurs only for wavelengths $\lambda_{B}$ satisfying:

$$
\Lambda=m\left(\lambda_{B} / 2 n_{\text {eff }}\right)
$$

where $\Lambda$ is the grating period, $n_{\text {eff }}$ is the average mode index, and the integer $m$ represents the order of Bragg diffraction. The coupling between the forward and backward waves is strongest for the fist-order Bragg diffraction $(m=1)$.

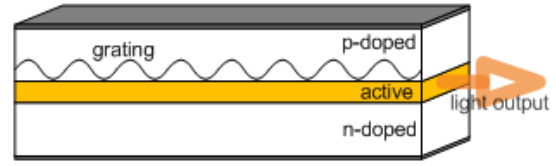

(a)

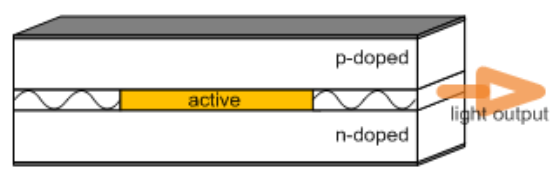

(b) 


\section{Figure 5. Side view of simplified DFB (a) and DBR (b) laser structures.}

Semiconductor lasers using the DFB mechanism canbe classified into two broad categories: DFB lasers and distributed Bragg reflector (DBR) lasers. Figure 5 shows the two kinds of structures. While in DFB lasers the feedback occurs throughout the cavity length, it does not take place inside the active region of a DBR laser. The fabrication of DFB lasers requires multiple epitaxial growths. A grating is etched onto one of the cladding layers surrounding the active layer. A thin n-type waveguide layer between the substrate and the active layer acts as a grating. The periodic variation of its thickness translates into a variation of the mode index $n_{\text {eff }}$ along the cavity length and leads to Bragg diffraction. Holographic techniques or electronbeam lithography are used to create the mask to fabricate the grating. Despite the technological complexities, DFB lasers are routinely produced commercially, and used in nearly all $1550 \mathrm{~nm}$ optical systems beyond $2.5 \mathrm{~Gb} / \mathrm{s}$. It must be noted that, although Figure 5 does not show any index-guiding structure, current available DFB lasers are designed following index-guiding principles.

Lasers can be modelled by a set by a set of differential equations that describe dynamically the number of electrons $(N)$ as well as the number of photons $(P)$ in the laser cavity:

$$
\frac{d P}{d T}=G P+R_{s p}-\frac{P}{\tau_{p}} \quad \frac{d N}{d T}=\frac{I}{q}-\frac{N}{\tau_{c}}-G P
$$

These equations are commonly known as the laser rate equations, and their derivation and thorough discussion can be found in references such as [AGR93]. Laser behaviour under modulation, noise, spectral linewidth, linearity and stability with temperature can be all predicted from the rate equations and building parameters of the laser.

The P-I (optical power vs. injected electrical current) curve can be derived from the rate equations and characterizes the emission properties of a semiconductor laser. This curve indicates not only the threshold level of current when stimulated emission begins to beat spontaneous emission, but also the current that needs to be applied to obtain a certain amount of power, which, in the linear zone above threshold, follows the relation:

$$
P=\eta\left(I-I_{t h}\right)
$$

where $I_{t h}$ and $\eta$ are, respectively, the threshold current and slope efficiency of the laser, while $I$ is the input current to the laser, and $P$ is the optical power at its output. 
Figure 6(a) is a typical example of a P-I curve, plotted at different temperatures. The observed degradation of threshold current and slope efficiency is known to have an exponential dependence with temperature:

$$
I_{t h}(T)=I_{0} \exp \left(\frac{T}{T_{0}}\right) \quad \text { and } \eta=\eta_{0} \exp \left(-\frac{T}{T_{1}}\right)
$$

That is, laser performance degrades with increasing temperature in two ways: increasing of threshold current and decreasing of slope efficiency $T_{0}$ and $T_{1}$ are called characteristic temperatures, and depend basically on the material used and the laser structure. Because of the temperature sensitivity of semiconductor lasers, it is often necessary to control their temperature through an attached thermo-electric cooler (TEC). Another known effect of temperature variations is the change in laser emission wavelength. Lasers that must work at a constant wavelength need to be stabilized in temperature with a TEC. Furthermore, operation of semiconductor lasers at a high temperature is equivalent to aging, and thus device reliability can decrease rapidly under these conditions. The main drawback of using a TEC is its high power consumption, which can be one order of magnitude higher than the consumption of the laser diode on which it is attached. The output of a semiconductor laser exhibits fluctuations in its intensity, phase and frequency even when the laser is biased at a constant current. Noise in semiconductor laser is dominated by spontaneous emission; each spontaneously emitted photon perturbs both amplitude and phase randomly. Intensity fluctuations lead to a limited signal-to-noise ratio, whereas phase fluctuations lead to a finite spectral linewidth.

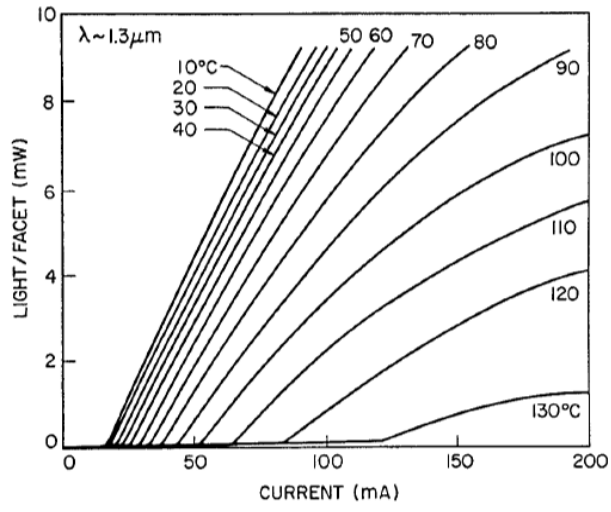

(a)

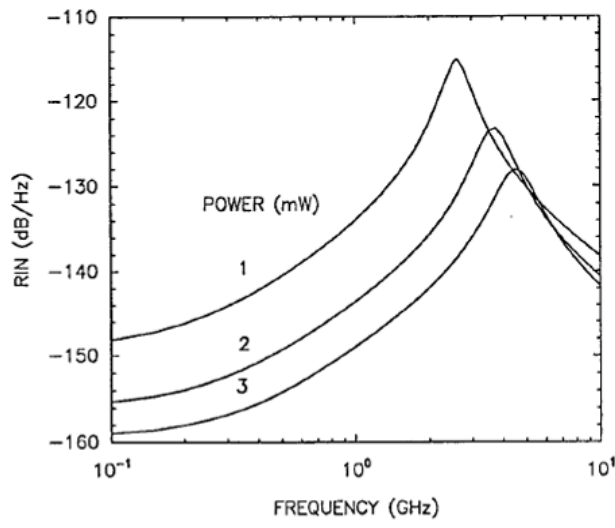

(b)

Figure 6. P-I curves at several te mperatures (a) and RIN spectra at se veral power le vels (b) of a typical 1550-nm semiconductor laser [AGR93]. 
The rate equations can be also used to study laser noise by adding a noise term, known as the Langevin force, to each of them. Its resolution [AGR02] leads to the definition of the relative intensity noise (RIN) of the laser, whose spectrum behaves in semiconductor lasers in a similar way to the one depicted in Figure 6(b). Derivation of RIN from rate equations is tricky, but some characteristics can be extracted from the graph. One is that RIN spectrum is not flat, but rather has a peak near the laser relaxation oscillation frequency (a couple $\mathrm{GHz}$ ), and decreases at lower frequencies. It must be pointed out, however, that, though not shown in this graph, RIN rises again at very low frequencies (below $10 \mathrm{MHz}$ ), where it shows a $1 / f$ behaviour [BIB99]. Other significant feature is that RIN decreases with increasing optical power.

Another important issue in optical transmitter design is how to couple as much light as possible from the laser diode into the optical fibre. Typical coupling efficiencies for edge-emitting lasers are 40-50\%. An important problem related to the laser-fibre coupling and laser packaging design is the sensitivity of semiconductor lasers to optical feedback. Even a feedback smaller than $0.1 \%$ can destabilize the laser, causing effects such as linewidth broadening and RIN enhancement. Demanding applications, in which most microwave photonic processing are included, will need the inclusion of an optical isolator between the laser and the fibre to reduce feedback. Optical isolators make use of the Faraday effect toblock light propagating into the laser. They achieve high isolation (> $30 \mathrm{~dB})$, low insertion loss and compact size, and are being used in most high-performance semiconductor lasers.

The use of external modulation of CW optical signals exhibits a higher electrical modulation bandwidth beyond the achievable with direct modulations of the laser power. It also improves the performance of the photonic link through increased linearity. External optical modulators (EOMs) are classified according to the parameter of the optical signal that is modulated. Phase modulators are commonly based on the Pockels effect of electro-optic crystals such as lithium niobate $\left(\mathrm{LiNbO}_{3}\right)$ while amplitude modulation is often performed by means of interferometric structures (Mach-Zehnder and Michelson) and phase modulators or materials with controllable absorption (electro-absorption modulators, EAM).

The Mach-Zehnder modulator (MZM) is widely employed for amplitude modulation. It is based on a Mach-Zehnder interferometer where the phase modulation performed on one or both arms is converted into amplitude modulation. In the Figure 4 the general case of both arms being modulated is included where the modulation voltage applied to the modulator is comprised of constant components $V_{D C l}$ and $V_{D C 2}$, and time-varying components, $V_{R F}(t)$. $V_{D C}$ is chosen to operate the modulator in different points of its transfer function according to the particular 
application. For example, data modulation requires linear responses and quadrature biasing $(\mathrm{QB})$ is needed. On the other hand, for nonlinear applications, maximum or minimum transmission biasing (MATB and MITB, respectively) provide better performance. Figure 7 shows the transfer function of a MZM. The half-wave voltage $\left(V_{\pi}\right)$, i.e. the voltage required to induce a $\pi$ radians phase shift between the arms of the interferometer, is a quality parameter of the modulator since it is related to the efficiency of the optical modulation. Small values of $\mathrm{V}_{\pi}$ are desired for increased efficiency.

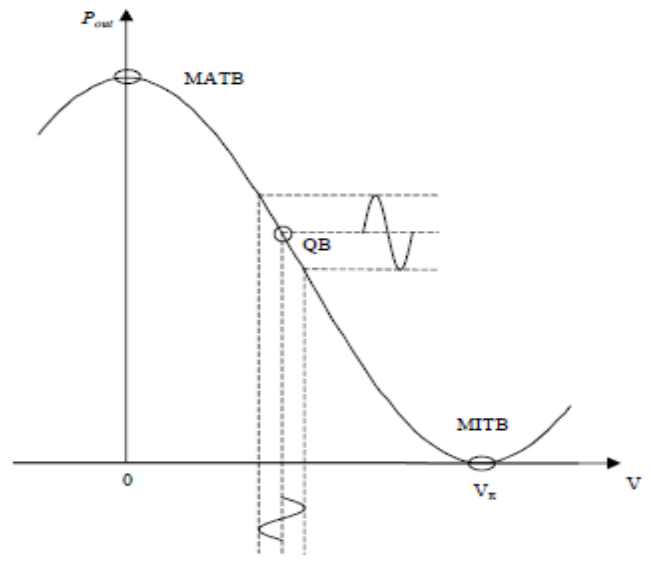

Figure 7. Sche matic of the Mach-Zehnder modulator transfer function.

An exhaustive analysis of the Mach-Zehnder modulator for the different modulation bias points can be found in [COR01]. For the investigations carried out in this thesis, in most of the cases the modulation bias point selected was Quadrature Bias (QB, which is the most linear modulation point) in configurations of dual-drive, push-pull (both modulator arms driven by the same signal with opposite phase). In this case, considering the following modulating signals with $\omega_{R F}$ being the modulating frequency:

$$
\begin{aligned}
& V_{1}(t)=V_{D C 1}+v_{R F} \sin \left(\omega_{R F} t+\phi_{R F 1}\right) \\
& V_{2}(t)=V_{D C 2}+v_{R F} \sin \left(\omega_{R F} t+\phi_{R F 2}\right)
\end{aligned}
$$

And the optical field at the output of the modulator is:

$$
E_{m z m}(t)=\sqrt{2 P t_{f f}} e^{j \phi_{c}} \sum_{n=-\infty}^{\infty} J_{n}\left(m_{R F}\right) \cos \left(\phi_{v}+n \phi_{d}\right) e^{j n\left(\omega_{R F} t+\phi_{m}\right)}
$$


where $J_{n}(x)$ is the Bessel function of first kind of order $n, t_{f f}$ are the insertion losses and

$$
\begin{gathered}
m_{R F}=\frac{\pi v_{R F}}{V_{\pi}} \\
\phi_{c}=\phi_{e}+\frac{\pi\left(V_{D C 1}+V_{D C 2}\right)}{2 V_{\pi}} \\
\phi_{m}=\frac{\phi_{R F 1}+\phi_{R F 2}}{2} \\
\phi_{v}=\frac{\pi\left(V_{D C 1}-V_{D C 2}\right)}{2 V_{\pi}} \\
\phi_{d}=\frac{\phi_{R F 1}-\phi_{R F 2}}{2}
\end{gathered}
$$

$m_{R F}$ is the optical modulation index and $\phi_{e}$ is the optical phase of the signal at the central frequency when nor voltage applied to the drivers. The propagation dispersive media from the optical modulator to the photodiode, in our case optical fibre or optical waveguides, can be modelled with a frequency response of

$$
H_{\text {link }}(\omega)=\frac{1}{\sqrt{L}} e^{j \beta(\omega)}
$$

where $L$ is the optical power loss of the dispersive link and $\beta(\omega)$ is the modepropagation constant according (1.2) and

$$
E_{\text {link }}(\omega)=E_{m z m}(\omega) H_{\text {link }}(\omega)
$$

is the optical field at the photodiode input. The photodetected current $\left(i_{p d}(t)\right)$ at the photodiode output follows the following expression:

$$
i_{p d}(t)=\frac{\Re P t_{f f}}{L}\left(I_{D C}+\sum_{p=1}^{\infty} I_{p \mathrm{RF}}(p)\right)
$$

where $\mathfrak{R}(\mathrm{A} / \mathrm{W}$ ) is the photodiode responsivity (normally between 0.4 and $0.9 \mathrm{~A} / \mathrm{W}$ ), $P(W)$ is the laser power, $L$ is the total optical losses from the laser to the photodiode, $I_{D C}$ is the term of the photocurrent at zero frequency and $I_{p \mathrm{RF}}(p)$ are the terms of the photocurrent of order $p$, The photodiode performs the operation of envelop detection 
of the optical field that arrives to it, particularly $i_{p d}(t)=\Re E_{\text {link }} E_{\text {link }}^{*} / 2$, being the photocurrent linear with the optical power. The photodiode is is a semiconductorbased detector coupled to an optical fibre or to an optical waveguide in the case of a PIC, and its electrical output is matched to the output circuitry to enable operation at high electrical frequencies (some photodiodes, UTC-PD, can reach the $\mathrm{THz}$ band). Optical absorption is the fundamental mechanism behind the photodetection process. The simplest photodetector consists of a semiconductor slab in which incident photons with energy exceeding the bandgap of the material generate an electron-hole pair. GaAs is employed for the $800 \mathrm{~nm}$ region while $\mathrm{Si}$ is suitable at $1 \mu \mathrm{m}$ and InGaAs compounds are used for the telecom band of $1.5 \mu \mathrm{m}$. Important parameters defining the performance of a PD are responsivity, bandwidth and dark current. Responsivity measures how efficient the photodetector is in terms of generated current per incident optical power. The dark current, which is the current generated in the absence of optical power, is the last important parameter of a PD and its value should be negligible.

There are several types of photodiodes. The most common is the p-i-n PD, which consists of an intrinsic layer placed between p- and n-doped layers. Good performance is provided by these structures despite of the existent trade-off between high responsivity and fast response. Avalanche photodiodes (APD) are suitable for low optical power operation since they provide larger responsivities thanks to a gain layer which generates additional electron-hole pairs through impact ionization. New designs of the epitaxial layers such as the uni-travelling-carrier photodiode (UTC-PD) have been proposed for increased bandwidth. In the UTC-PD [ISH97] the absorption takes place in the p-layer instead of the intrinsic. Because pair electrons-holes are generated in the p-layer the transit time of the holes is really short and the electrons are the ones limiting the speed of the PD. This device has shown high-power operation at speeds exceeding $1 \mathrm{THz}$ with high linearity suitable for data generation.

As commented, the particular case of $\mathrm{QB}$ modulation results from $\mid V_{D C I^{-}}$ $V_{D C 2} \mid=\mathrm{V}_{\pi} / 2$ and $\left|\phi_{D C 1^{-}} \phi_{D C 2}\right|=\pi$. In this case, the different components of the detected signal from (1.16) may be simplified resulting in

$$
I_{D C}(t)=\frac{1}{2}
$$




$$
\begin{aligned}
I_{p R F}(p)= & \cos \left(p \omega_{R F}\left(t+\theta_{1}\right)+p \phi_{m}\right) \\
\cdot & {\left[J_{p / 2}^{2}\left(m_{R F}\right)+2(-1)^{p / 2} \sum_{n=(p / 2)+1}^{\infty} J_{n}\left(m_{R F}\right)\right.} \\
& \left.\cdot J_{n-p}\left(m_{R F}\right) \cos \left(\frac{\theta_{2}}{2} p(2 n-p) \omega_{R F}^{2}\right)\right]
\end{aligned}
$$

for even harmonics of the RF signal ( $p$ even) and

$$
\begin{aligned}
I_{p R F}(p)= & \pm 2 \cos \left(p \omega_{R F}\left(t+\theta_{1}\right)+p \phi_{m}\right) \\
& \cdot \sum_{n=(p+1) / 2}^{\infty}(-1)^{n-(p-1) / 2} J_{n}\left(m_{R F}\right) J_{n-p}\left(m_{R F}\right) \\
& \left.\cdot \cos \left(\frac{\theta_{2}}{2} p(2 n-p) \omega_{R F}^{2}\right)\right]
\end{aligned}
$$

for fundamental and odd harmonics of RF signal ( $p$ odd) [COR01]. $J_{n}$ are the Bessel functions of first kind of order $n$.

The electrical power associated to the fundamental component of the RF signal, $P_{R F}$, can be simplified as:

$$
P_{R F}=2 \Re^{2} P^{2} g_{\text {link }}^{2} J_{0}^{2}\left(m_{R F}\right) J_{1}^{2}\left(m_{R F}\right) R_{L}
$$

where $g_{\text {link }}$ is the total optical gain of the link equal to $1 / L$ and $R_{L}$ is the load impedance of the photodetector. For $m_{R F} \ll 1$, the Bessel functions can be approximated as:

$$
\begin{gathered}
J_{0}\left(m_{R F}\right) \cong 1, \\
J_{1}\left(m_{R F}\right) \cong m_{R F} / 2
\end{gathered}
$$

and expression (1.19) can be simplified to:

$$
P_{R F}=\frac{1}{2} \Re^{2} P^{2} g_{\text {link }}^{2} m_{R F}^{2} R_{L}
$$


From Eq. (1.21), the required modulation index $m_{R F}$ needed to obtain a specified $\mathrm{RF}$ detected power can be expressed as a function of the rest of system parameters as:

$$
m_{R F}=\sqrt{\frac{2 P_{R F}}{\mathfrak{R}^{2} P^{2} g_{\text {link }}^{2} R_{L}}}
$$

The optical modulation index can be related to the RF input power applied to each drive of the Dual-Drive MZM as:

$$
P_{R F-i n}^{D D}=\frac{\left(V_{R F}\right)^{2}}{2 \cdot Z_{I N}}=\frac{V_{\pi}^{2} \cdot m_{R F}^{2}}{2 \cdot \pi^{2} \cdot Z_{I N}}
$$

However, if a Single-Drive MZM is used, the required RF input power for a given $m_{R F}$ is:

$$
P_{R F-i n}^{S D}=\frac{\left(2 V_{R F}\right)^{2}}{2 \cdot Z_{I N}}=\frac{2 \cdot V_{\pi}^{2} \cdot m_{R F}^{2}}{\pi^{2} \cdot Z_{I N}}
$$

\subsubsection{Carrier-to-noise Ratio}

The signal-to-noise ratio (SNR) is a parameter that characterises the quality of a device, system, or link. The system performance depends on the SNR associated with the demodulated signal. In the case of sub-carrier multiplexing (SCM) systems, the carrier-to-noise ratio (CNR) is often used instead of SNR, as the two are related to each other depending of the electrical modulation. The CNR is defined as the ratio between the desired carrier component and the overall noise at the output of the system under analysis. In this section, the main noise contributions are described and an expression of the CNR for a generic microwave photonic system is provided.

Figure 8 shows a schematic view of the noise link model which has been employed to analyse the CNR. All the main noise sources which have some influence in the performance of the system have been included in the model. A single laser source which generates an optical carrier with a power $P_{\mathrm{i}}$ has been considered. The losses $L_{1}$ before the first amplifier include the insertion losses of the MZM and the first optical circulator, whilst the losses $L_{2}$ between amplifiers take into account the insertion losses of the TTD matrix and finally, $L_{3}$ include the second circulator and the demultiplexer insertion losses. The main noise sources are described next. 


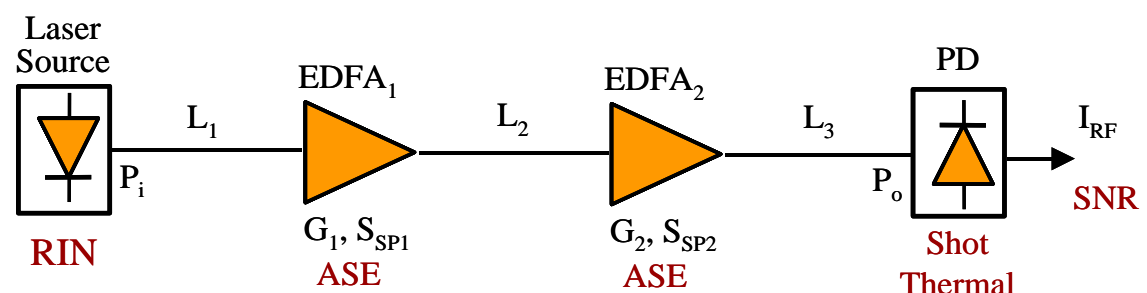

Figure 8. Noise link model schematic.

\subsubsection{Thermal noise}

The thermal noise, whose variance will be denoted by $\sigma_{t h}^{2}$, is due to random thermal motion of electrons in the load resistor placed in the front end an optical receiver. Its expression is given by:

$$
\sigma_{t h}^{2}=\frac{4 k_{B} T \Delta f F_{n}}{R_{L}}
$$

where $k_{B}=1.384 \cdot 10^{-23} \mathrm{~K} / \mathrm{J}$ is the Boltzmann constant, $T$ is the absolute temperature, $\Delta f$ is the effective noise bandwidth which is fixed by the last filtering stage, $F_{\mathrm{n}}$ is the amplifier noise figure due to several resistors used in pre- and main amplifiers and $R_{\mathrm{L}}$ is the load resistance.

It should be noted that the equivalent noise bandwidth for all noise components has been set to the equivalent electrical noise bandwidth which is fixed by the latter filtering stage after the photoreceiver, e.g. by the RF amplifier or an electrical filter.

\subsubsection{Shot noise}

The shot noise is due to the random nature of the electrons that form the electrical current after the photodetection process. The total shot noise variance is given by,

$$
\sigma_{s}^{2}=2 q\left(I_{p}+I_{d}\right) \Delta f
$$

where $q$ is the electron charge $q=1.6 \cdot 10^{-19} \mathrm{C}, I_{\mathrm{p}}$ is the average photocurrent and $I_{\mathrm{d}}$ is the dark current.

\subsubsection{RIN noise}

This noise component depends on the value of the relative intensity noise (RIN) parameter of the laser. Assuming that it is nearly uniform within the receiver bandwidth, the RIN noise term after detection is given by, 


$$
\sigma_{R I N}^{2}=R I N I_{p}^{2} \Delta f
$$

It should be noted that the RIN noise has a quadratic dependence with the average receiver optical intensity.

\subsubsection{EDFA noise}

Erbium-doped fibre amplifiers (EDFA) are widely used in today's optical communication systems to boost optical carriers or to compensate for losses in optical links and devices. However, the amplified signal is contaminated by the spontaneous emission that takes place during the amplification process. The noise terms are due to an increase in the shot noise due to the spontaneous emission, and the beating of spontaneous emission against itself, signal and shot noise. In our system there are two different ASE (Amplified Spontaneous Emission) sources, but as they are not placed just before the photodetector, it is necessary to study the variation suffered by these noise contributions as they travel from their source to the photodetector. The first amplifier, denoted as $\mathrm{EDFA}_{1}$, gives rise to a spontaneousemission noise whose spectral density $S_{\mathrm{sp} 1}$ can be written as:

$$
S_{s p 1}(v)=\left(G_{1}-1\right) n_{s p 1} h v
$$

where $v$ is the optical frequency, $G_{1}$ is the optical gain, $n_{\mathrm{sp} 1}$ is the populationinversion factor, and $\mathrm{h}$ is the Planck constant $\left(\mathrm{h}=6.626 \cdot 10^{-34} \mathrm{~J} \cdot \mathrm{s}\right)$. The noise spectrum amplitude is modified from the output of $\mathrm{EDFA}_{1}$ to the input of the photodetector where the new spectral density $S_{\text {sp } 1}^{\prime}$ can be expressed as:

$$
S_{s p 1}^{\prime}(v)=\frac{G_{2}}{L_{2} \cdot L_{3}} S_{s p 1}(v)
$$

If the same analysis is applied to the second amplifier, denoted as EDFA 2 , the following expressions are obtained:

$$
\begin{gathered}
S_{s p 2}(v)=\left(G_{2}-1\right) n_{s p 2} h v \\
S_{s p 2}^{\prime}(v)=\frac{1}{L_{3}} S_{s p 2}(v)
\end{gathered}
$$

where $S_{\mathrm{sp} 2}$ and $S_{\text {sp2 }}$ are the spectral densities of the spontaneous-emission noise originated by $\mathrm{EDFA}_{2}$ at the amplifier output and at the photodetector input, respectively. 
Taking into account the previous considerations, the noise term corresponding to Shot noise is affected by the ASE noise as follows:

$$
\sigma_{s}^{2}=2 q\left(\Re\left(P_{o}+S_{s p 1}^{\prime} \cdot \Delta v+S_{s p 2}^{\prime} \cdot \Delta v\right)+I_{d}\right) \Delta f
$$

where $P_{\mathrm{o}}=P \mathrm{i} \cdot G_{1} G_{2} /\left(L_{1} L_{2} L_{3}\right)$ is the optical power of the desired signal at the input of the photodetector, and $\Delta v$ is the amplifier bandwidth, which is supposed to be identical for both amplifiers.

The noise terms due to the beating of spontaneous emission against itself, signal and shot noise are:

$$
\begin{aligned}
& \sigma_{s p-s p 1}^{2}=4 \Re^{2}{S^{\prime}{ }_{s p 1}}^{2} \Delta v \Delta f \\
& \sigma_{s i g-s p 1}^{2}=4 \Re^{2} P_{o} S^{\prime}{ }_{s p 1} \Delta f \\
& \sigma_{s-s p 1}^{2}=4 q R^{\prime} S_{s p 1}^{\prime} \Delta v \Delta f
\end{aligned}
$$

for the noise generated at $\mathrm{EDFA}_{1}$ and:

$$
\begin{gathered}
\sigma_{s p-s p 2}^{2}=4 \mathfrak{R}^{2} S_{s p 2}^{\prime 2} \Delta v \Delta f \\
\sigma_{s i g-s p 2}^{2}=4 \Re^{2} P_{o} S_{s p 2}^{\prime} \Delta f \\
\sigma_{s-s p 2}^{2}=4 q \Re^{\prime}{ }_{s p 2} \Delta v \Delta f
\end{gathered}
$$

for the noise generated at $\mathrm{EDFA}_{2}$. The noise term due to the beating of the spontaneous emissions of both amplifiers has been neglected.

The total noise term after photodetection $\sigma^{2}$ can be obtained by adding the individual noise terms previously obtained:

$$
\begin{aligned}
\sigma^{2}=\sigma_{t h}^{2}+ & \sigma_{s}^{2}+\sigma_{R I N}^{2}+\sigma_{s p-s p 1}^{2}+\sigma_{s i g-s p 1}^{2}+\sigma_{s-s p 1}^{2}+ \\
& +\sigma_{s p-s p 2}^{2}+\sigma_{s i g-s p 2}^{2}+\sigma_{s-s p 2}^{2}
\end{aligned}
$$

The carrier-to-noise ratio CNR can be obtained from Eq. (2.1) and Eq. (2.22) as:

$$
C N R=\frac{P_{R F}}{\sigma^{2}}
$$




\subsection{Photonic Up and Down Conversion}

The generalization of the intensity modulation, direct detection microwave photonic link depicted in Figure 4 is shown in Figure 9, [COR01]. In this case, the $\mathrm{CW}$ laser source is generalized to an optical source that is formed by an externally modulated optical carrier, resulting in cascading two MZM modulators. In this case, the optical dual-side band modulation generated by this optical source is again modulated by a second MZM modulator. This second modulation stage results in a complex optical spectrum that, after the photodetector, generate a number of harmonic components including harmonics of an eventual intermediate frequency component (IF), harmonic of the signal modulating the second MZM, named here Local Oscillator in order to maintain the nomenclature of the frequency converters, and mixing product between both $\pm n \cdot I F \pm p \cdot L O$. The proper selection of the desired spectral component by an filter result in a functionality of frequency conversion, either up, down conversion, or even harmonic frequency conversion of higher order.

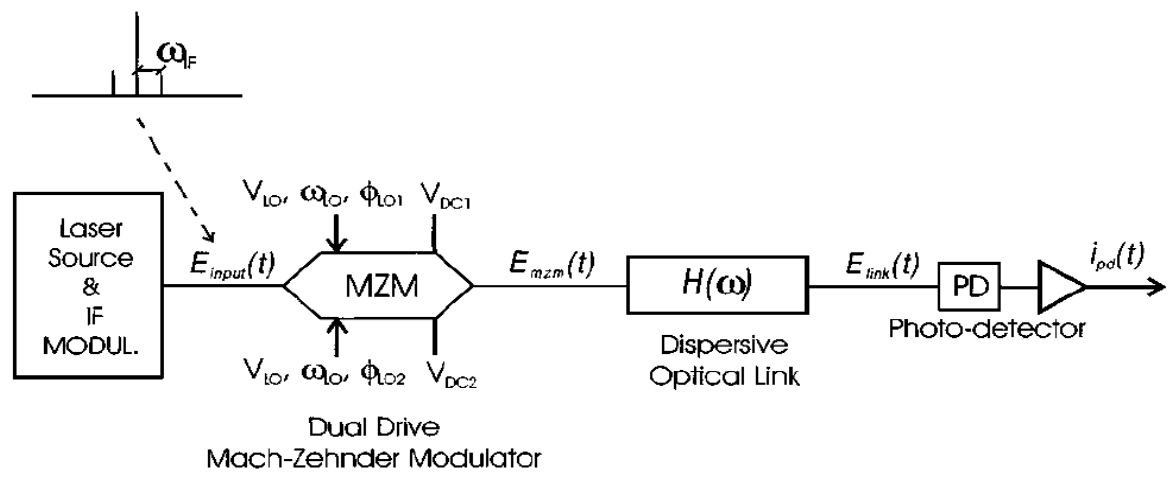

Figure 9. Optical fre quency convers ion of an IF modulated optical signal with an optical MZM modulator in a dispersive link [COR01].

In Figure 10, an example of frequency conversion done with a system as described in Figure 9 with an IF frequency of $3.5 \mathrm{GHz}$ and a LO frequency of 25 $\mathrm{GHz}$ is shown. The harmonics of the IF carrier, the fundamental of the $\mathrm{LO}$ and the frequency conversions of $\mathrm{LO} \pm n I F$ are shown, both by simulation and by measurements. The frequency conversion in this kind of systems is free of spurious different from the harmonic products of the MZM input signals, which does not occur with the electrical mixers, which exhibits spurious in frequencies not directly associated to the input signals. This particular behaviour is very useful when a broadband frequency converter is required and is unique of the microwave photonic systems. 

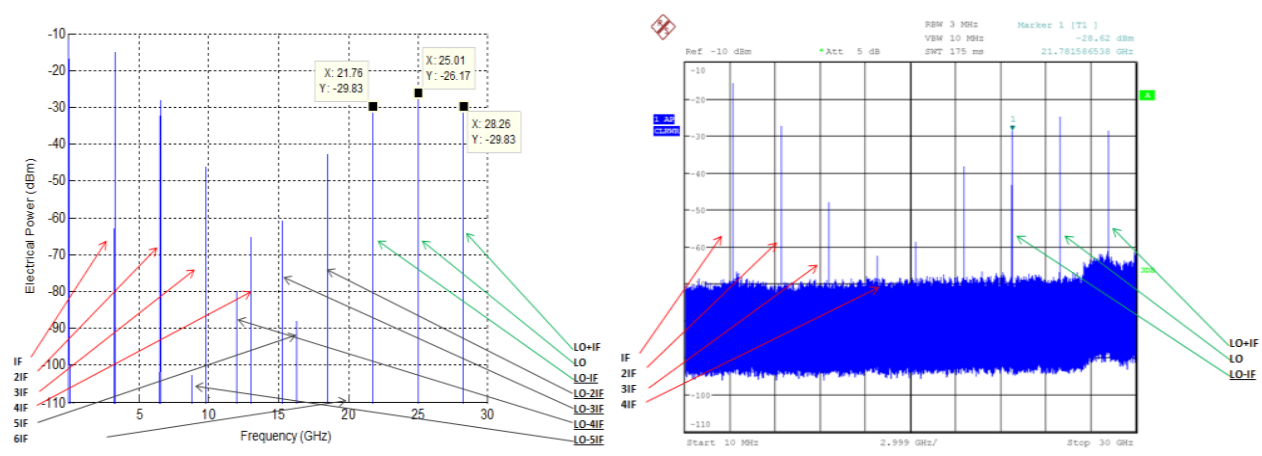

Figure 10. Ele ctrical s pectrum of a microwave photonic fre que ncy converter at the output of the photodiode. Left picture shows a simulation and righ one is the real meas urement.

The equations that govern the behaviour of a photonic frequency converter, as the one described above, can be found in [COR01]. The concrete case for the up and down converted components (pLO+IF) and (pLO-IF) respectively is the following:

$$
\begin{aligned}
& I_{p L O+I F}(p)= m_{I F} \cos \left(\frac{\theta_{2}}{2} \omega_{I F}\left(p \omega_{L O}+\omega_{I F}\right)\right) \\
& \cdot \sum_{n=-\infty}^{\infty} I(n) I(n-p) \cdot \cos \left(\left(p \omega_{L O}+\omega_{I F}\right)\left(t+\theta_{1}\right)+p \phi_{m}+\phi_{I F e}\right. \\
&\left.+\frac{\theta_{2}}{2}(2 n-p) \omega_{L O}\left(p \omega_{L O}+\omega_{I F}\right)\right) \\
& I_{p L O-I F}(p)= m_{I F} \cos \left(\frac{\theta_{2}}{2} \omega_{I F}\left(p \omega_{L O}-\omega_{I F}\right)\right) \\
& \cdot \sum_{n=-\infty}^{\infty} I(n) I(n-p) \\
& \cdot \cos \left(\left(p \omega_{L O}-\omega_{I F}\right)\left(t+\theta_{1}\right)+p \phi_{m}-\phi_{I F e}\right. \\
&\left.+\frac{\theta_{2}}{2}(2 n-p) \omega_{L O}\left(p \omega_{L O}-\omega_{I F}\right)\right)
\end{aligned}
$$

being $\theta_{n}=L \beta_{n}$ as in (1.2-5), and 


$$
I(n)=J_{n}\left(m_{R F}\right) \cos \left(\phi_{v}+n \phi_{d}\right)
$$

There is a particular case in which, if the frequency of the IF signal is lower than $2.5 \mathrm{GHz}$ typically, the first modulation can be done by the direct modulation of the bias current of a laser. The previous equations describe the general case in which the first modulation (IF) is ideal (dual-side band) so the particular case of a directly modulated laser is also included.

\subsection{Vector Microwave Optical Processing}

As described in Section 1.3, photonics offers a new approach to distribute and control microwave signals. Optical fibre can provide low loss to transport microwave signals over long distances with improved flexibility in comparison to coax cables. Additionally, the amplitude of the microwave signal can be controlled (e.g. [YAO98], [COX04]). The phase of the microwave signal can also be modified by using optical phase shifters (e.g. [LOA06]).

Some applications require the simultaneous control of both the amplitude and the phase/delay of a microwave signal, i.e. assuming a monochromatic signal, the control over the signal phasor, complex amplitude or vector components of the signal. Photonic architectures can control both the amplitude and the phase/delay of microwave signals with the key feature of ultrabroadband operation. The phase at the output of the photodiode can be modified by changing the delay that the modulated optical signal suffers by crossing an optical circuit. This delay, as will be seen in subsequent sections, can be changed with different techniques. The amplitude can also be controlled by different means ranging from the reduction of the optical power emitted by a laser, reduction of the gain of an amplifier or by a dedicated optical or electrical attenuator.

This thesis focuses on the application of photonic vector control of radiofrequency signals in two applications: optical beamforming and photonic vector modulation and demodulation of digital quadrature amplitude modulations. Photonic vector control enables to adjust the amplitude and phase of the radiofrequency signals in the optical domain.

\subsubsection{Optical Beamforming Networks}

Antenna arrays consist of a set of individual antenna elements working simultaneously as a single antenna of larger dimensions and with some features not easily achievable in elemental antennas such as electronic control of the steering angle. Arrays are based on the constructive/destructive interference of the signals 
emitted by their elements. The unique feature of this solution is that the antenna steers the angular direction of maximum radiation only with the proper adjustment of the differential phases of the signals at the antenna elements. This process can be parallelized and multiple beams can be emitted/received and independently steered with the proper beamforming network in what is known as multibeam antennas. The major drawback of these solutions is that the complexity of the beamforming network increases dramatically with the number of elements of the antenna, impacting, in turn, on the size, mass, volume, consumption and reliability of the beamforming network.

The work described in this thesis includes different techniques for implementing a photonic version of beamforming networks for DRAs, known as optical beamforming networks (OBFN). The optical beamforming technology has attracted the interest of the research community for more than two decades owing to the fact that this technology present an attractive solution not only for overcoming the conventional issues for implementing RF true time-delay (TTD) in broadband applications, but because it also allows the distribution of RF signals to the radiating elements, benefiting from the features of optical fibre as transmission medium, i.e. low mass, small volume, low attenuation, flexibility, broad bandwidth, immunity to electromagnetic interference (EMI), and improved phase stability.

Several optical beamforming architectures have been proposed until now using different technological implementations [ZMU94]. In particular, optical TTD beamforming has been proposed using free-space optics, fibre optics or integrated optics. Optical TTD based on free-space optics have been considered almost since the early days for their potential for parallel processing. On the other hand, they may require significant free-space propagation lengths (especially at low RFfrequencies), creating collimation loss and beam diffraction issues, and thereby limiting the performance, stability and overall scalability of the BF system.

Fibre-based optical delay lines avoid these problems and offer much lower loss, yielding better performance. Most of the options make use of the numerous fibrebased components developed for the telecom industry including optical modulators, amplifiers and switches, and wavelength management devices (e.g., filters, demultiplexers, dispersive fibres).

Integrated optics based photonic beamformers are of particular interest from the point of view of compactness and moderate implementation costs. Their attractiveness is expected to increase as the RF signal frequency increases.

The research carried out for this thesis has follows the objective of, firstly, to address a precise control in terrestrial applications of broadband signals at very high 
frequencies above $40 \mathrm{GHz}$ in communication antennas and, secondly, to optimize the size and mass when compared with the electrical or optical counterparts in space application. In the first case true time-delay control is used for the phase adjustment of broadband signals in dispersive optical fibres with wavelength division multiplexing techniques for parallelization. Additionally, techniques to provide advanced features such as direction of arrival estimation in receiving architectures are studied. In the second, passive OBFNs based on monolithically-integrated Optical Butler Matrices are studied.

\subsubsection{Photonic Vector Modulator for Communications}

Broadband wireless access (BWA) networks are key last-mile-access technologies. BWA facilitates extending network coverage with relatively low deployment costs, faster revenue growth, and increased flexibility compared to common cabled infrastructure. In parallel, wired local area networks (LAN) and fibre-to-the-home (FTTH) access networks, are experiencing a remarkable capacity increase, as both CAPEX and OPEX costs are decreasing due to higher market penetration, standardization and use of low-cost optical technologies. This increasing capacity of wired data transmission has pushed the development of wireless technologies capable of transmitting high data rate signals, which is only possible by utilizing the millimetre-band $(\mathrm{mmW})$. During the past few years, substantial knowledge about the $\mathrm{mmW}$ communication has been accumulated and a great deal of work has been done toward developing systems for commercial applications [HIR06], [DYA07], [RID08].

However, these schemes in the $\mathrm{mmW}$ band require a huge electrical bandwidth (more than $20 \mathrm{GHz}$ for $10 \mathrm{~Gb} / \mathrm{s}$ ), resulting in a very limiting option in frequency division duplex schemes. Also the complexity of technology both electrical and optical at these high frequencies $(>100 \mathrm{GHz})$ limit the possibilities.

In this thesis, techniques for controlling the phase and amplitude of radiofrequency signals (i.e. vector processing) have been also investigated to be applied to the generation quadrature digital modulations. Multilevel modulation is a very well-known technique of the transmission of digital signals that is universally used in communications, from mobile phones to communication satellites. The fundamental performance of this technique is that the digital information is encoded in discrete states of phase and amplitude of an electrical signal (vector). Having two states, encoded either in the phase ( \pm 180 degrees) or the amplitude (0-1), is the simplest case. The general case is known as quadrature modulation in which both amplitude and phase changes in discrete values. In this general case, the modulation is decomposed in its two orthogonal components phase-shifted 90 degrees, known as in-phase and quadrature components. The signal processing required for 
generating and demodulating this kind of signals involves vector processing (phase and amplitude control) and frequency conversion.

In this thesis, this signal processing to generate and receive digital modulations is optically performed in a technique known as photonic vector modulation (PVM) and demodulation (PVdM). This is of particular interest in the case of broadband requirements where the data-rate required to be managed is in the order of gigabits per second, for applications as wireless backhauling of metro optical networks (also known as fibre-to-the-air). The proposed techniques use optical dispersion in optical fibres, wavelength division multiplexing and photonic up/down conversion. Additionally, an optical heterodyne solution implemented in highly integrated CMOS compatible photonics for this application scenario is described including silicon modulators combined with integrated photonics and CMOS compatible photo-detection. 


\section{CHAPTER 2}

\section{Optical Beamforming Networks}

\subsection{Introduction to Antenna Theory}

A phased array antenna is made of multiple stationary antenna elements, which are fed coherently and use variable phase or time-delay control at each element to scan a beam at given angles in space [MAI05]. Sometimes, variable amplitude control is also provided for radiation pattern shaping. Arrays are routinely used instead of fixed aperture antennas (reflectors, lenses) because the multiplicity of elements allows more precise control of the radiation pattern, thus resulting in lower sidelobes or careful pattern shaping. However, the primary reason for using arrays is to produce a directive beam that can be repositioned (scanned) electronically, with application in radar, communication, sensing, etc.

The sketch in Figure 11 portrays a generalized distribution of array elements, here shown as small radiating surfaces. Each element radiates a vector directional pattern that has both angle and radial dependence near the element. 


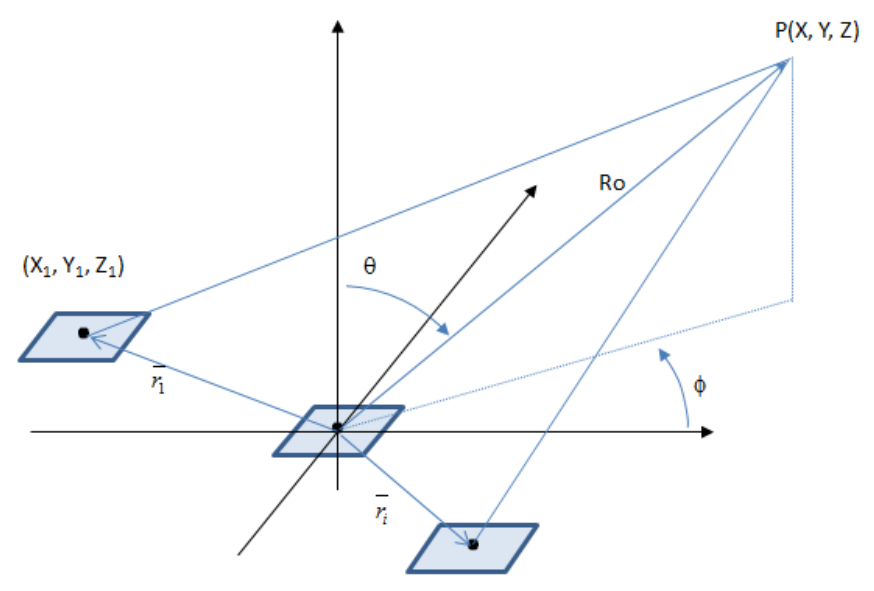

Figure 11. Array and coordinate systems.

The radiation behaviour of the array can be characterized by the scalar array factor $F(\theta, \phi)$, where:

$$
F(\theta, \varphi)=\sum a_{i} \mathrm{e}^{j k \vec{r}_{i} \cdot \hat{r}}
$$

\subsubsection{Scanning and Collimation of Linear and Planar Arrays}

Array scanning can be accomplished by applying the complex weights $a_{i}$ in the form of:

$$
\begin{gathered}
a_{i}=\left|a_{i}\right| \mathrm{e}^{-j k \vec{r}_{i} \cdot \hat{r}_{0}} \\
\hat{r}_{0}=\operatorname{sen}\left(\theta_{0}\right) \cos \left(\phi_{0}\right) \hat{x}+\operatorname{sen}\left(\theta_{0}\right) \operatorname{sen}\left(\phi_{0}\right) \hat{y}+\cos \left(\theta_{0}\right) \hat{z}
\end{gathered}
$$

These weights steer the beam peak to an angular position $\left(\theta_{0}, \phi_{0}\right)$, because at that location the exponential terms in (2.2) cancel those in (2.1), and the array factor is the sum of the weight amplitudes $\left|a_{i}\right|$. With this choice of weights, the pattem maximum is stationary for every frequency. This requires an exponential dependence that has a linear phase relationship with frequency that corresponds to inserting time delays or lengths of transmission line among the path followed by the signal in its travel to each antenna element. These delays are chosen so that the path length differences for the generalized array locations of Figure 11 are compensated in order to make the signals from all elements arrive together at some desired distant point.

Due to the difficulty in providing time delays, the steering signal can be controlled by phase shifters, which are much simpler to implement and are a good 
approximation in the case of limited bandwidth, instead of using actual time delays. In this case, the weights have the form below instead of that in (2.2):

$$
a_{i}=\left|a_{i}\right| \mathrm{e}^{-j k_{0} \vec{r}_{i} \cdot \hat{r}_{0}}
$$

for some frequency $f_{0}=c / \lambda_{0}$ (with $k_{0}=2 \pi / \lambda_{0}$ ). In this form, the array pattern has its peak at a location that depends on frequency.

\subsubsection{Bandwidth}

The bandwidth of an array antenna [KIN72, KNI74] can be limited by the bandwidth of each one of the elements forming the array, but often the limiting effect is due to the use of phase shifters to scan the beam instead of time-delay devices. The complex weights shown in (2.2) provide time delay, and so the beam peakoccurs at $\left(\theta_{0}, \phi_{0}\right)$ for all frequencies. If phase shifters are used instead to steer the beam, the peak is scanned to the desired angle only at centre frequency $f_{0}$. Otherwise, it is steered to that angle which makes the exponent of (2.4) equal and of opposite sign to the exponent of (2.1).

The resulting radiation diagram shows a phenomenon known as "beamsquint", like that depicted in Figure 12(a), in which the beam peak angle is reduced for frequencies above the design frequency and increased for frequencies below the design frequency. If the bandwidth is defined by the frequency limits at which the gain is reduced to half power, the resulting fractional bandwidth given by the beamsquint is given by:

$$
\frac{\Delta f}{f}=\frac{\theta_{a}}{\sin \left(\theta_{0}\right)}=0.886 B_{b}\left(\frac{\lambda}{L \sin \left(\theta_{0}\right)}\right)
$$

for an array with beamwidth $\theta_{\mathrm{a}}$ and beam broadening factor $B_{b}$. The bandwidth becomes smaller as the array is made larger or as the scan angle is increased. Figure 12(b) shows bandwidth versus scan angle for arrays of different lengths. 

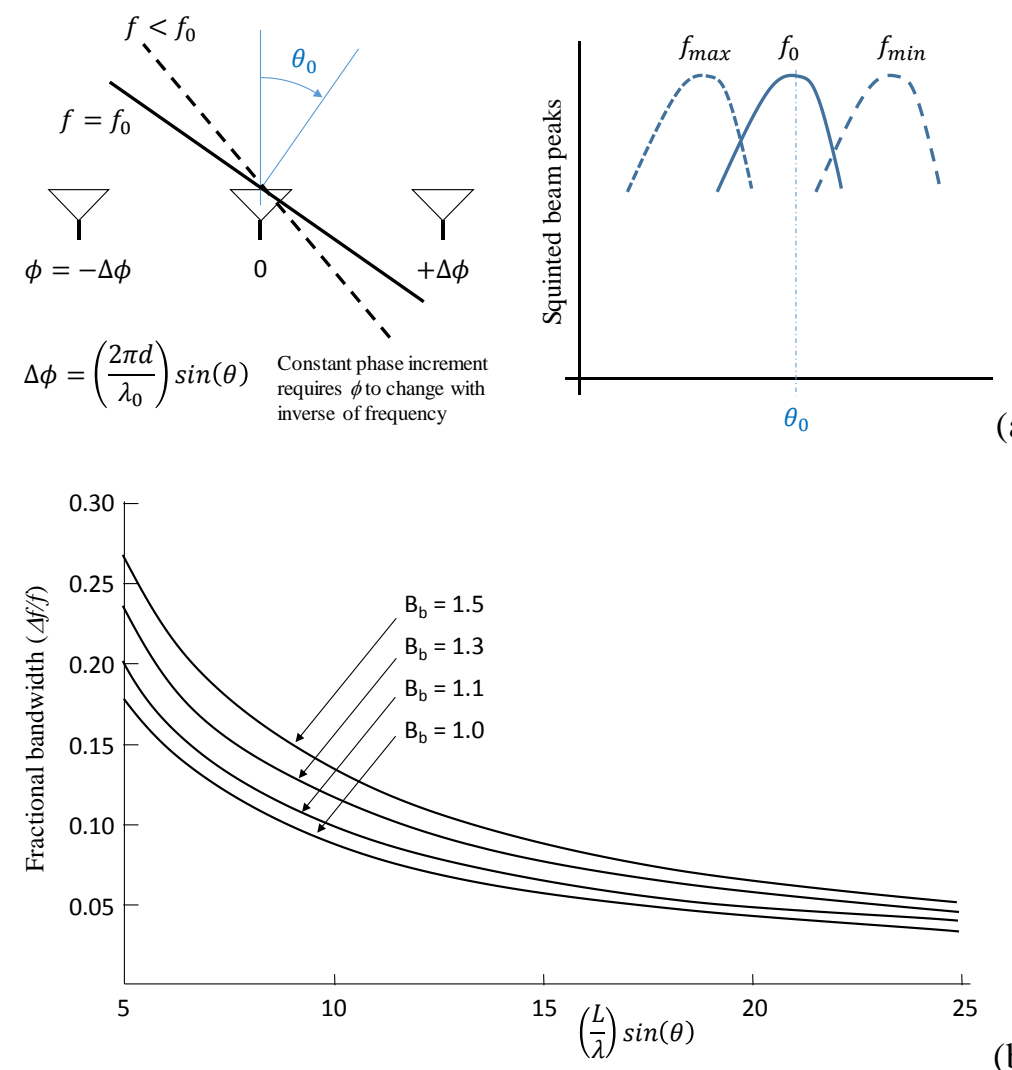

(b)

Figure 12. Wide band effects in phase d array performance: (a) be am s quint for a phased array (wavefronts and beam peak motion); (b) array 3-dB bandwidth vers us $(L / \lambda) \sin \left(\theta_{0}\right)$ ( $B b$ is beam broadening factor) [KIN72].

\subsubsection{Time-Delay Compensation}

The bandwidth limitation imposed by (2.5) severely restricts the use of arrays in several applications such as military radar, next generation Earth observation instruments and broadband communication systems. The use of time delays instead of phase shifts, an approach known as True Time Delay (TTD), can give enhanced bandwidth, but often at prohibitively large cost and at the expense of other performance goals.

In order to maintain the beam peak at a constant angle $\theta_{0}$ for all frequencies, one needs time delayed signals at eachelement. The excitation coefficients for anequally spaced $\left(d_{x}\right)$ linear array are given by: 


$$
a_{n}=\mathrm{e}^{-j\left(\frac{2 \pi}{\lambda}\right) n d_{x} \sin \left(\theta_{0}\right)}=\mathrm{e}^{j \Phi_{n}}
$$

In terms of equivalent phase $\Phi_{n}$ at each element, these phase shifts are:

$$
\Phi_{n}=-2 \pi \frac{n f}{c} d_{x} \sin \left(\theta_{0}\right)
$$

and thus need to vary linearly with frequency.

The common method to provide time delay is to insert incremental lengths of transmission line of length $L_{n}=n d_{x} \sin \left(\theta_{0}\right)$ to produce the time delays,

$$
\tau_{n}=\frac{n d_{x} \sin \left(\theta_{0}\right)}{c}=\frac{L_{n}}{c}
$$

For example, it can be done by switching sections of transmission lines behind each element or group of elements. Since the phase shift inserted by length of line $L_{n}$ is:

$$
\Phi_{n}=\frac{2 \pi L_{n}}{\lambda}
$$

each line length (near the ends of the array with total length $L$ ) has to be variable over the range

$$
\frac{-L}{2} \sin \left(\theta_{0}\right) \leq L_{n} \leq \frac{L}{2} \sin \left(\theta_{0}\right)
$$

In this case, the negative value does not indicate a negative line length, since an equal length of line is first added to each path.

The beam steering angle provided by the TTD $\tau_{n}$ is given by,

$$
\theta_{i}=\arcsin \left(2 \cdot \tau_{n} \cdot f_{R F}\right)
$$

However, the required lengths of switched microwave transmission lines are extremely bulky and expensive for large arrays, and the large number of discrete time-delay positions requires a highly complex switching network. Furthermore, the relative dispersion in the various transmission line sections may prohibit accurate beam forming. For these reasons there are few deployed systems that rely on time delay controls, and to date these have been large ground-based arrays in very specific 
niche applications mainly related with the military. In space, a relevant example of antenna system that implements true time-delay sections is the beamforming network of the Cosmo-SkyMed SAR satellite [TOR11].

The need for wideband array systems is increasing, and analogue, optical, and digital technologies can provide that function, each one with its limitations.

\subsubsection{Multiple Beam Array Feeds}

A special category of array feed is the multiple beam array shown schematically in Figure 13, where each input port excites an independent beam in space. These can be produced with a digital beamformer, but in addition there are a variety of antenna hardware concepts that produce multiple beams, including optical technologies.

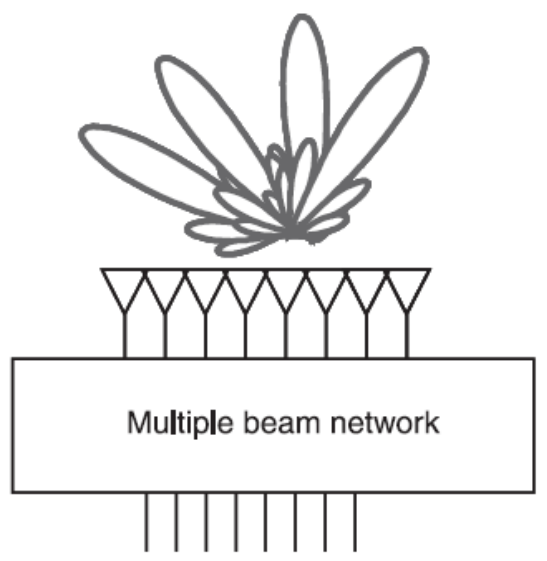

Figure 13. Multiple beam array.

Since the 50's, the Butler Matrix [BUT61], [BUT85] has been known by antenna specialists as a device based on cascading couplers so that any RF signal from a given input is distributed with equal amplitude to eachoutput, and with a progressive phase-shift from an output to the next. So, when connecting the output ports to the elements of an antenna linear array, the signal injected in each input port is radiated in a pre-determined direction, within an 'antenna directive beam'. All beams formed by a Butler matrix are equally spaced (provided that $\sin (\theta)$ is taken as angular unit) and 'orthogonal', which is a fundamental property that allows no crosstalk between them. This orthogonality is because a Butler matrix (see Figure 14) is a circuit implementation of the fast Fourier transform (FFT) and radiate orthogonal sets of beams with uniform aperture illumination. It is a passive reciprocal network, so it works the same when used in transmitting or receiving mode. Butler matrices can be combined in two steps (cascade) to facilitate 3D scanning. 

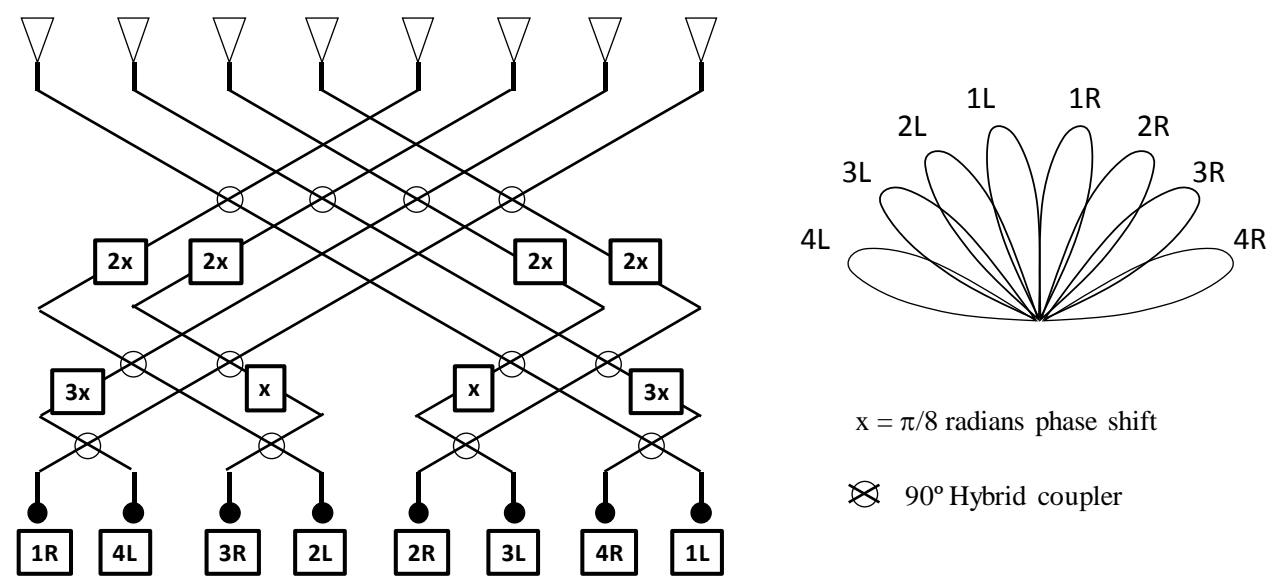

Figure 14. Eight-element, eight-beam Butler matrix and radiated be ams.

Besides, the Butler matrix is the waveguide network able to provide $\mathrm{N}$ beams from $\mathrm{N}$ ports (at input and outputs) with the minimum number of couplers: $\mathrm{N} \cdot\left(\log _{2} \mathrm{~N}\right) / 2$, instead of $2 \mathrm{~N} \cdot(\mathrm{N}-1)^{3}$ for a traditional beamformer with independent ways for phase-shifting towards outputs the signal dedicated to each beam, either in microwave circuits or possibly connecting by optical fibres eachinput to each output.

Despite this great hardware reduction, a Butler matrix network is rather bulky if built on RF waveguide technology. When it has to connect numerous inputs to the same number of $\mathrm{N}$ outputs, especially as for bidimensional arrays, this needs two successive sets of stacked Butler matrices, as presented in Figure 15, for the case of $\mathrm{N}=8^{2}=64$, being ' $\mathrm{N}$ ' the overall number of ports (at input and output) of a 2D set of Butler Matrices. In the classical implementation, it is composed of 2 sets of stacked planar matrices, which will be said of MxM order. As the optimal Fourier transformers, the optimal implementation of a $\mathrm{BM}$ is for $\mathrm{M}=2^{\mathrm{m}}$ and a matrix can be truncated to use a number of inputs and/or outputs $<2^{\mathrm{m}}$.

${ }^{3}$ In a classical BFN with independent forming per beam, at each of the $\mathrm{N}$ inputs, a $1 \rightarrow \mathrm{N}$ divider needs N-1 couplers (e.g. 3dB-ones if uniform distribution); at outputs, also N-1 couplers are necessary to combine signals coming from each 'BFN slide' located in front of each of the $\mathrm{N}$ outputs. 

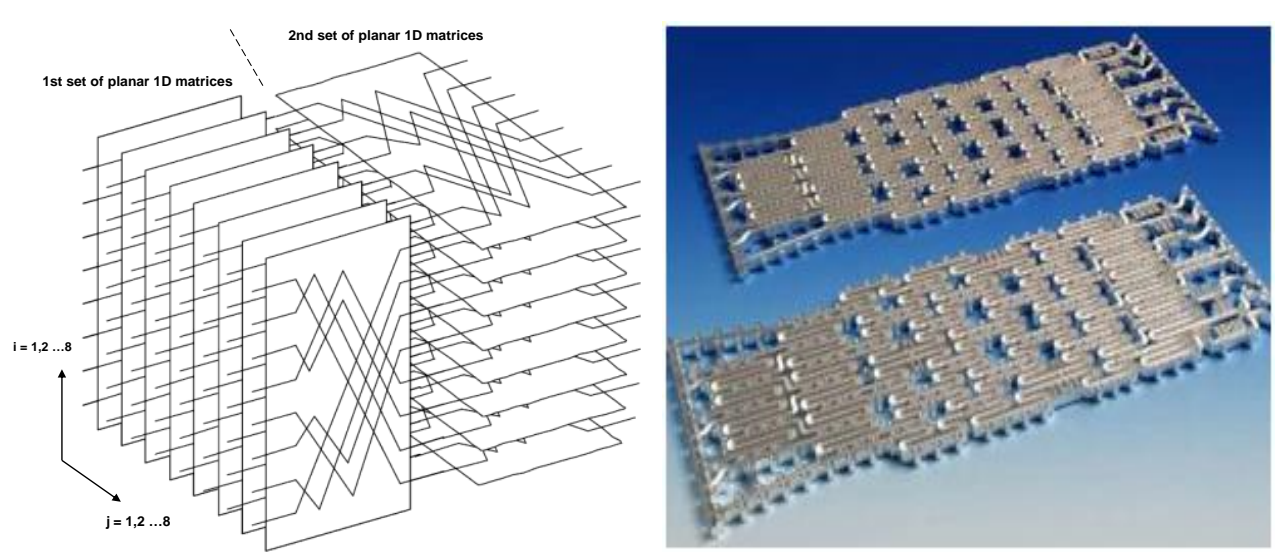

Figure 15. Microwave B utler Matrixes. (le ft) 3D Orthogonal double set of 8x8 B utler matrixes and (right) example of a $8 \times 8 \mathrm{RF}$ matrix at $30 \mathrm{GHz}\left(15 \times 8 \mathrm{~cm}^{2}\right)$.

Because the beams of a matrix-fed array phase are scanned, they show inherently modest bandwidths. On the other side, multiple beam lens and reflector systems have the advantage of being wideband scanners, as their beam locations do not vary with frequency since each beam is geometrically dependent of the position of discrete illuminators. In present antenna systems, as for example on-board of communication satellites, an array of illuminators (normally made by horn antennas after a high power amplifier) are placed at the focus of a single parabolic reflector or a set of reflectors in a Cassegrain configuration and each illuminator is in charge of generating each antenna beam. This configuration is quite simple and widely used since, as described before, is theoretically a wideband system. Nevertheless, there are drawbacks associated to the reliability of the RF chain of each illuminator since if an illuminator fails the whole beam coverage is lost. This is not the case in phase array systems since the loss of a single element has low impact in the radiation pattern (this is especially positive in antenna arrays of a high number of elements) ${ }^{4}$. Other practical limitation is that the linearity of the high-power amplifiers associated to each illuminator is normally worse than medium-power amplifiers used in array antennas (since the power of each beam is distributed among all the antenna elements). This is a practical limitation in broadband systems in which a high number of carriers needs to be amplified together, generating distortion.

An alternative to reflector systems in one-beam-per-illuminator systems is the Rotman lens [ROT63], a variant of the Gent bootlace lens [GEN57] that has the special feature of forming three points of perfect focus for one plane of scan. The Rotman lens can provide good wide-angle scanning out to angles exceeding 45 degrees. It consists of an artificial lens formed by two set of illuminators

\footnotetext{
${ }^{4}$ See Annex B.
} 
confronted. The first set corresponds to each beam and the second to each antenna element. The geometry of this arrangement is the origin of the pointing angle of each beam following the same principle of a typical optical lens. This configuration can be seenimplemented in microstrip technology for linear arrays or bi-dimensional for planar arrays. The following figure shows a Rotman lens in planar configuration.

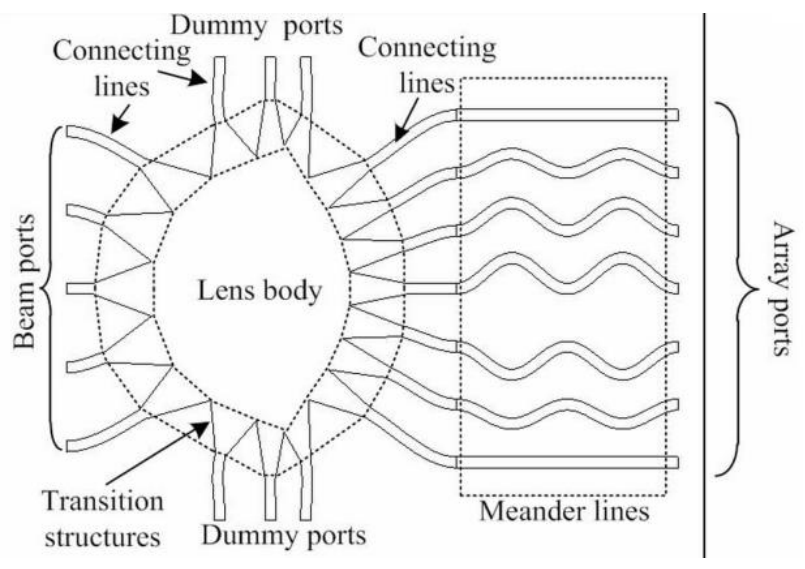

Figure 16. Rotman le ins beamforming network.

Multiple beam lenses and reflectors have been chosen for satellite communication systems, and in that application they serve to produce either switched individual beams or clusters of beams to cover particular areas on the Earth.

\subsubsection{Control for Wideband Arrays}

As it has been seen in previous sections, beamsquint, as illustrated in Figure 12 and (2.5), requires the use of time-delay steering in very wideband arrays as well as in very large arrays with even modest fractional bandwidth. These two categories of wideband arrays are distinctly different and require completely different architectures.

Figure 17 and Figure 18 show several approaches on how to implement time delay networks for various relevant conditions. Figure 17 shows two possible architectures for very wideband (octave or multioctave) or multiple-band control. The diagram of Figure 17a shows one T/R module and one-time delay unit (TDU) per element and provides exact time delay and the ultimate bandwidth subject to antenna element design. The T/R amplification at the elements is necessary because TDUs are lossy (depending on their length and implementation technology). 


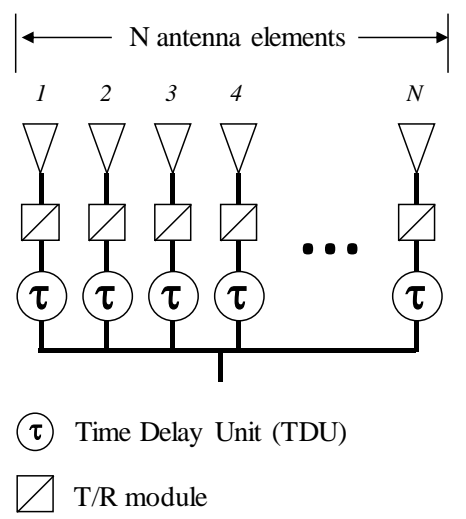

(a)

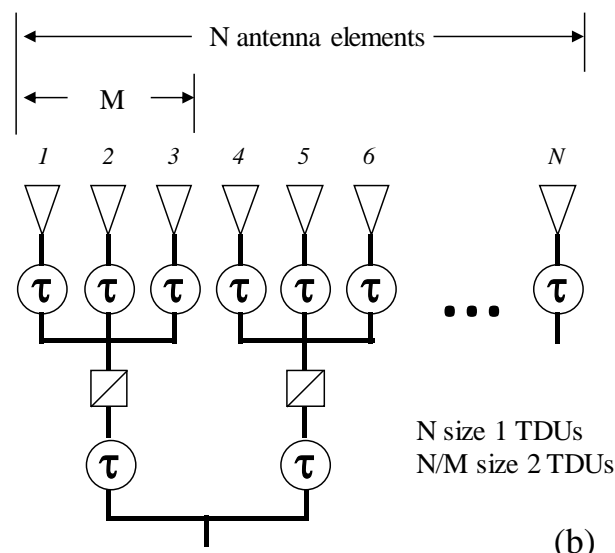

(b)

Figure 17. Wide band array control: (a) array with TDUs; and (b) array with cascaded TDUs.

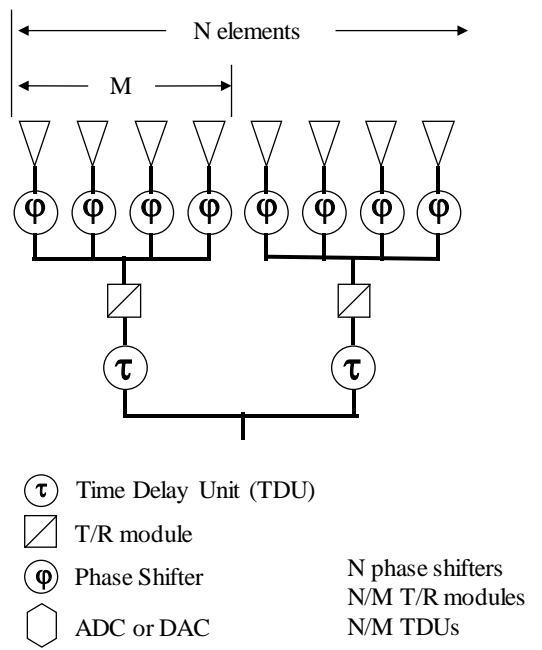

(a)

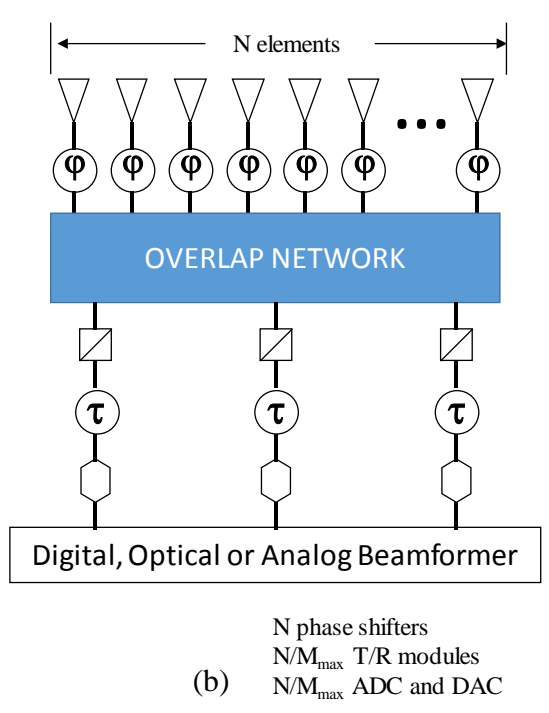

Figure 18. Archite ctures for fractional bandwidth wide band arrays: (a) phased array with contiguous time-delayed subarrays; and (b) phased array with time-delaye d overlappe d subarrays.

From eq. (2.10) it can be stated that an array that is 100 wavelengths long needs nearly 100 wavelengths of excess line switched in series with the outermost elements for scan angles up to 60 degrees; thus, significant loss can be expected. In addition to loss, there is little room behind each element to include the TDUs and amplification, so this most basic of architectures is impractical for most applications except for relatively small, very wideband arrays using traditional RF technology. 
However, a photonic approach could ease these limitations by benefiting from the fact that losses in optical fibre are almost independent of the length of the delay line.

The right side of Figure 17(b) shows a more practical configuration for providing element-level time delay and, like the first, provides the exact time delay at every element. This configuration provides small increments of time delay at each element, perhaps up to two or three wavelengths. Then, after grouping these elements into subarrays and amplifying, it provides longer delays at successive levels of subarraying. Very long delays can then be provided by a beamformer using optical, analogue, or digital time delay. In this case, the optical and analogue time delay could be provided by a switched line configuration; thus, it retains the wideband features of the basic apertures. At present, the performance of digital beamformers do not support octave or multioctave bandwidth at microwave frequencies, but they can provide accurate time delay over narrower bandwidths at a multitude of frequencies through subbanding and filtering. In these cases, the digital beamformer can provide multiband beams that point in the same direction using the network of cascaded TDUs.

\subsection{Review of Optical Beamforming Networks}

\subsubsection{Antenna Beamforming Networks}

Optical beamforming networks for phased-array antennas [SEE93], [ZMU94], [VID04], [TUR09] have many potential advantages over their electrical counterparts such as small size, low weight, no susceptibility to electro-magnetic interference and, especially, wide instantaneous bandwidth and squint-free array steering (true time delay). This section reviews the state-of-the-art in photonic beamforming concepts and technologies, and their potential application in multi-beam antennas (MBA).

The application of photonic technology to beamforming has been investigated for more than three decades, [KOE84] [EST87] [ANA88] [NG91] [LEI99] [PAU99], bearing the promise of overcoming the conventional RFbeamformer issues that have been briefly described in the previous section. Photonic technology can not only generate effectively the time delays or phase shifts required to MBA, but also support the distribution of the RF signals to the radiating elements, with optical fibre advantages and reducing the complexity at the antenna, requiring less space behind the antenna elements, resulting in lighter antenna arrays.

There are presently two main technical approaches in optical beamforming for multibeam antennas: 
- Optical True Time-Delay (TTD) systems, which introduce frequency-independent time delays by optical propagation means, and thus inherently feature large bandwidths as described above.

- Phase-Shifting coherent optical beamformers, which are based on optical heterodyning and accurate phase control of the optical carrier to produce phase shifts on the microwave signals.

These concepts make use of various technologies, including free-space or semi-guided optics, and guided-wave optics. Table 2 provides a tentative classification of optical beamforming concepts and technologies.

In particular, optical TTD beamforming has been proposed using free-space optics, fibre optics or integrated optics. Optical TTD based on free-space optics have been considered, almost since the early days, for their potential for parallel processing. On the other hand, they may require significant free-space propagation lengths (especially at low RF frequencies), creating collimation loss and beam diffraction issues, and thereby limiting the performance, stability and overall scalability of the BF system.

Fibre-based optical delay lines avoid these problems and offer much lower loss, yielding better performance. Most of the architectures make use of the numerous fibre-based components developed for the telecom industry including optical modulators, amplifiers and switches, and wavelength management devices (filters, demultiplexers, dispersive fibres...). In the end, optical TTD units with discrete fibre optics components might result in bulky, or at least, non-optimized implementations.

Integrated optics based photonic beamformers can address these issues and broaden the market potential of photonic technology in beamforming networks providing compactness and moderating implementation costs. Their attractiveness is expected to increase as the RF signal frequency increases.

Recent coherent optical beamformer implementations most often use either a single spatial light modulator (SLM), or photonic integrated circuits on various substrates (Lithium Niobate, InP semiconductor, Silica, Silicon on Insulator, ...) with electro-optic or thermos-optic phase modulators instead of microwave phase shifters.

Since most applications do not require full TTD control, attempts have also been made to combine TTD and phase control to lower costs. This is achieved for instance by providing TTD to sub-arrays and controlling the relative phase between the elements of each sub-array. Thus, the beamsquint problem is significantly reduced for a given bandwidth while alleviating beamforming complexity. 
As also shown in the table, out of the many approaches to photonic beamforming, some are attempts to mimic in optics conventional RF beamformers. In particular, when it comes to offer multi-beam forming with fixed-angle pointing, optical beamformers can make use of optical Rotman lens, Blass or Butler matrices.

TABLE 2: A TENTATIVE Class IFICATION OF CONCEPTS AND TECHNIQUES FOR OPTICAL BEAMFORMING

\begin{tabular}{|c|c|c|}
\hline & $\begin{array}{c}\text { Free-space and } \\
\text { semi-guided-wave optics }\end{array}$ & $\begin{array}{c}\text { Guide-wave optics (fibre and } \\
\text { integrated optics) }\end{array}$ \\
\hline $\begin{array}{l}\text { Optical true } \\
\text { time-delay } \\
\text { beamformer }\end{array}$ & $\begin{array}{ll}\text { - } & \text { Free-space optical } \\
\text { delay lines BF } \\
\text { - MOEMS-based optical } \\
\text { delay lines BF } \\
\text { - Optical Rotman lens- } \\
\text { based BF } \\
\text { - Optical Blass matrix- } \\
\text { based BF }\end{array}$ & $\begin{array}{ll}\text { - } & \text { Fibre Optic Delay Line BF } \\
& \text { (FODEL) } \\
\text { - } & \text { Optical Fibre Rotman lens } \\
\text { - } & \text { Esman Fibre dispersion prism } \\
\text { - } & \text { FBG-based dispersion prism } \\
\text { - } & \text { Programmable dispersion } \\
& \text { matrix-based BF } \\
\text { - } & \text { Grating-based WDMBF } \\
\text { - Integrated optical delay line BF } \\
\text { - Optical ring resonator based } \\
\text { integrated BF }\end{array}$ \\
\hline $\begin{array}{l}\text { Coherent } \\
\text { optical } \\
\text { beamformer }\end{array}$ & 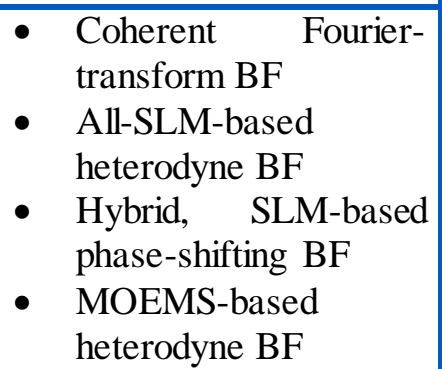 & $\begin{array}{l}\text { - BF based on integrated phase- } \\
\text { shifter on Lithium-Niobate, } \\
\text { Silica, or InP substrates }\end{array}$ \\
\hline
\end{tabular}

In the following, the main optical beamforming implementations for phased array antennas are reviewed, and a brief analysis is given regarding a number of aspects like insertion loss, accuracy, mass, size, power consumption, complexity and technology restrictions, suitability for multi-beam or beam-hopping applications. 


\subsubsection{True Time-Delay Optical Beamforming}

The main optical TTD devices include free-space or semi-guided optic delay lines, fibre optic delay lines (FODEL) using single-mode fibre with low or high dispersion, and fibre Bragg gratings (FBG). The optical TTD beamformer may be arranged as a so-called prism structure with low and high dispersion optical fibre or as a matrix structure.

\subsubsection{Free-space and semi-guided-wave optical delay line beamformers}

Free-space OBFNs typically use Spatial Light Modulators (SLMs) to control a parallel set of optical channels that can be independently controlled. By assigning each one of these channels to one photodiode/antenna element, the radiation pattern can be steered with TTD. Dolfi's architecture [DOL95] shown in Figure 19 is a well-known example of a free-space, 2D TTD beamformer based on polarization-switched delays using SLM. The emitted signal is channelized and for each channel, the beam goes through the pixels of $N$ SLM's. Each pixel acts as a voltage-driven polarization rotator which combined with the polarization beam splitter (PBS), switches the beam along one of the two paths. $N$ units provide time-delays in a geometric progression $\left(1 T, 2 T, \ldots, 2^{(N-1)} T\right)$ with $T$ being the time increment. The time delay pattern among output ports determines the far-field steering angle.

The first demonstration was achieved at 2.5 and $3.5 \mathrm{GHz}$, with 6 SLMs of $4 \times 4$ pixels providing 16 channels with 5-bit delays [DOL95]. A similar demonstration [VOD03] was achieved by replacing part of the components with fibre optics devices, and a compact 8-channel TTD unit operating in the $6-18 \mathrm{GHz}$ band was fabricated. An angular coverage of 90 degrees was achieved with 9 beam positions by using a compact 5-bit optical TTD, with delays from 6.4 to 178.4 ps.

A different approach is based on providing only phase control to exploit the relatively large degree of parallelism of SLMS. The collimated linearly polarized beam is focused inside an acousto-optic modulator (AOM). At the output of the AOM two angular spread beams are obtained whose angular frequency is $\omega$ and $\omega+2 \pi f$, respectively. The angular separation of the two beams allows that an N-SLM can phase modulate one of them. In addition the pixelated structure of the N-SLM allows the correspondence between one pixel and one fibre, in other words between one pixel and one antenna element. One of the main drawback of this proposal is that AOM offer poor bandwidth, frequency range and modulation index compared to conventional external modulators such as MZM [RIZ92]. 


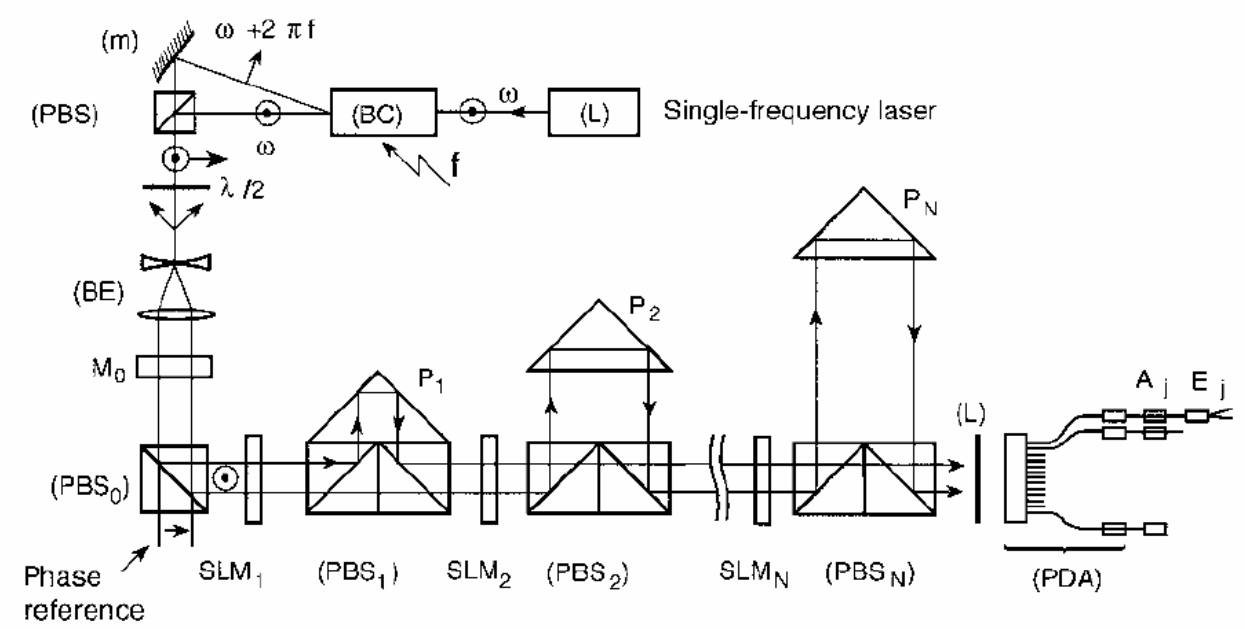

\section{Figure 19. Free-space, 3D TTD beamforme r bas ed on SLM matrix [DOL95].}

Photonic implementations of a Rotman-lens have also been proposed [CUR95] [ZAL09]. In [ZAL09], the photonic unit consists of a slab waveguide very similar in design as its RF counterpart with photodetectors as interfaces connected to the transmitting antennas, and whose shape can realize a linear phase profile with a varied slope, that is obtained at the lens output for any possible position at the lens input. The photonic configuration is claimed to have the advantage of a simplified module with reduced mass and volume.

Optical realizations of the Blass matrix based on a substrate-guided wave true time-delay (TTD) module have been proposed [LI00] [CHE02] as well. For example, in [CHE02] a 6-bit optical TTD module designed as an 8x8 two-dimensional time delay matrix providing time delays ranging from 0 to $443.03 \mathrm{ps}$ is demonstrated. The volume of the module is $90 \times 88 \times 4.63 \mathrm{~mm}^{3}$. This TTD module was packaged and tested in an eight-element K-band phased-array antenna system with operation at 18 to $26 \mathrm{GHz}$.

Figure 20 shows and approach based on the generation of TTD by micro-electromechanical-system (MEMS) micro-mirror array which allows a light beam to circulate among four spherical mirrors for a fixed number of round trips. The mirrors of the MEMS micro-mirrors array can tip to three angles (-10, 0 and +10 degrees). In case that all micro-mirrors are tipped to - 10 degrees the light beam is confined between mirrors $\mathrm{A}$ and $\mathrm{B}$. However, the light coming from $\mathrm{B}$ can be sent to $\mathrm{C}$, if the micro-mirror is tipped at +10 degrees. Next, the MEMS can either send the beam back to $\mathrm{C}$ or return it to $\mathrm{B}$ and $\mathrm{A}$. This can happen on every bounce on the MEMS. On the last bounce, the MEMS are programmed so that the beams land on the output 
turning mirror. A given time delay can be achieved by selecting how many times light bounces into mirror $\mathrm{C}$ [RAD03].

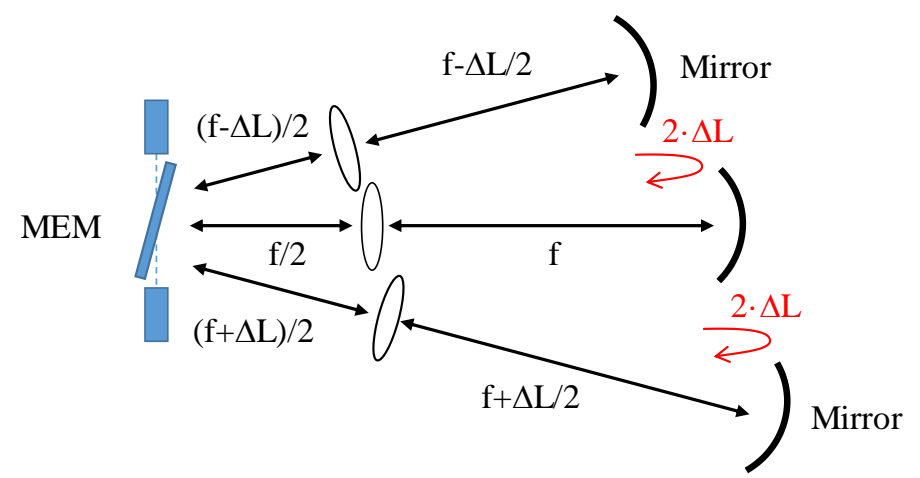

Figure 20. Free space TTD design based on MEMS mirrors.

\subsubsection{Fibre-optic delay line beamformers}

Programmable time-delay devices can be created using short lengths of single-mode fibre [SOR84] based on light propagation delay $\left(\beta_{1}\right)$. A well-known concept is the use of propagation delay and optical switches in what is known as a binary programmable fibre-optic delay line (BIFODEL) architecture shown in Figure 22 [GOU90]. The optical signal is routed through cascaded $2 \times 2$ optical switches through $\mathrm{N}$ fibre-optic delay lines, whose length increases with a geometric progression. The BIFODEL concept requires one TTD per antenna channel what reduces its overall potential for practical use in large MBA. To reduce the number of BIFODEL, one approach is to use a partition of the antenna aperture into sub-arrays and to use phase-shifters within the sub-arrays to have a limited beamsquint.

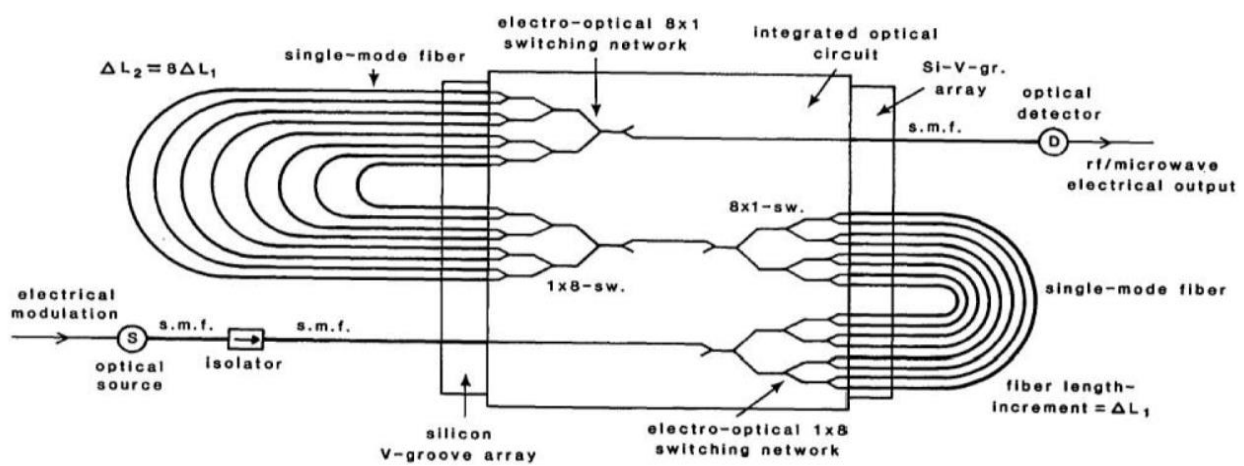

Figure 21. B inary programmable fibre-optic de lay line architecture [SOR84]. 
The use of semiconductor optical amplifiers (SOA) and flexible fibre circuit technologies was also proposed [MAD10]. A SOA can provide fast switching speed ( $10 \mathrm{~ns})$ and high on/off isolation required for beam-hopping, while the fibre-flex technology provides the required time delays with fine accuracy. It allows for very accurate control of the fibre lengths and differential delays. Currently, accuracy of $0.5 \mathrm{~mm}$ or better can be accomplished, so that a delay accuracy of $2.5 \mathrm{ps}$ or smaller is expected. For small delay times, the delay line shall be implemented by integrated optics.

A large-scale, three-dimensional micro-electro-mechanical-system (MEMS) optical switch was also used in a TTD beamformer for phased-array radar applications, with a capacity of 32 antenna elements and 8-bit delay [KAM03]. The $288 \times 288$ optical switch provided 82.944 paths with less than $2.3 \mathrm{~dB}$ loss, and switching time about $10 \mathrm{~ms}$.

Passive optical TTD beamformers can be designed as optical fibre implementations of a Rotman lens [ALA95], [SPA98], [SPA99]. Figure 23 shows an architecture of such an optical TTD BF for a receive MBA. It comprises an optical fibre delay line interconnect with appropriate lengths so as to form $\mathrm{M}$ independent beams in different directions from the $\mathrm{N}$ antenna array elements. In such fibre optic architectures, it is easy to use electro-optic modulators with semiconductor lasers for the RF-to-optical conversion, with bandwidth beyond $30 \mathrm{GHz}$, or to benefit from developments in advanced integrated laser-modulator devices, which make them attractive for high-frequency, large-scale beamformers. The beamformer may incorporate optical amplifiers before the optical splitters, with beneficial impact on system performance, compensating for the $\mathrm{BF}$ losses, and improving noise figure and dynamic range.

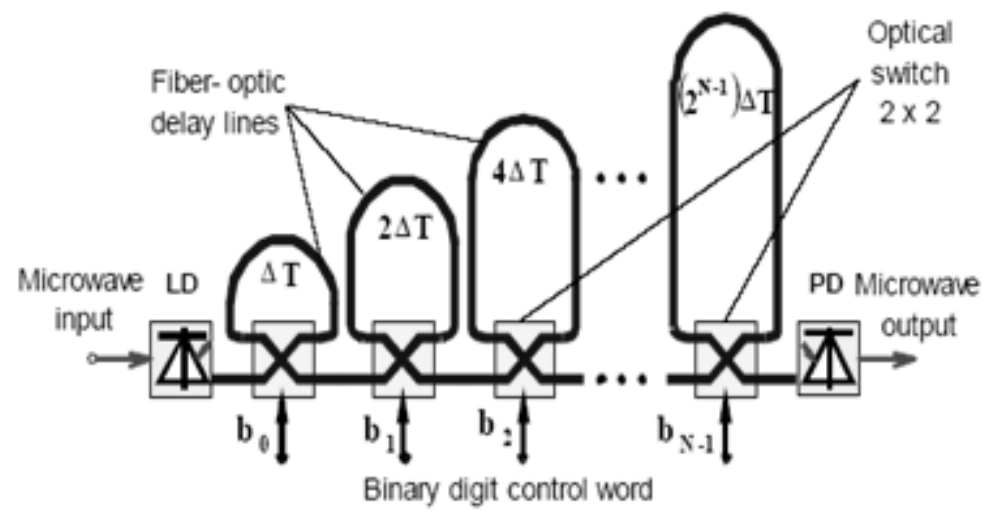

Figure 22. The binary fibre-optic delay line (BIFODEL) architecture based on s witched single mode fibres. 


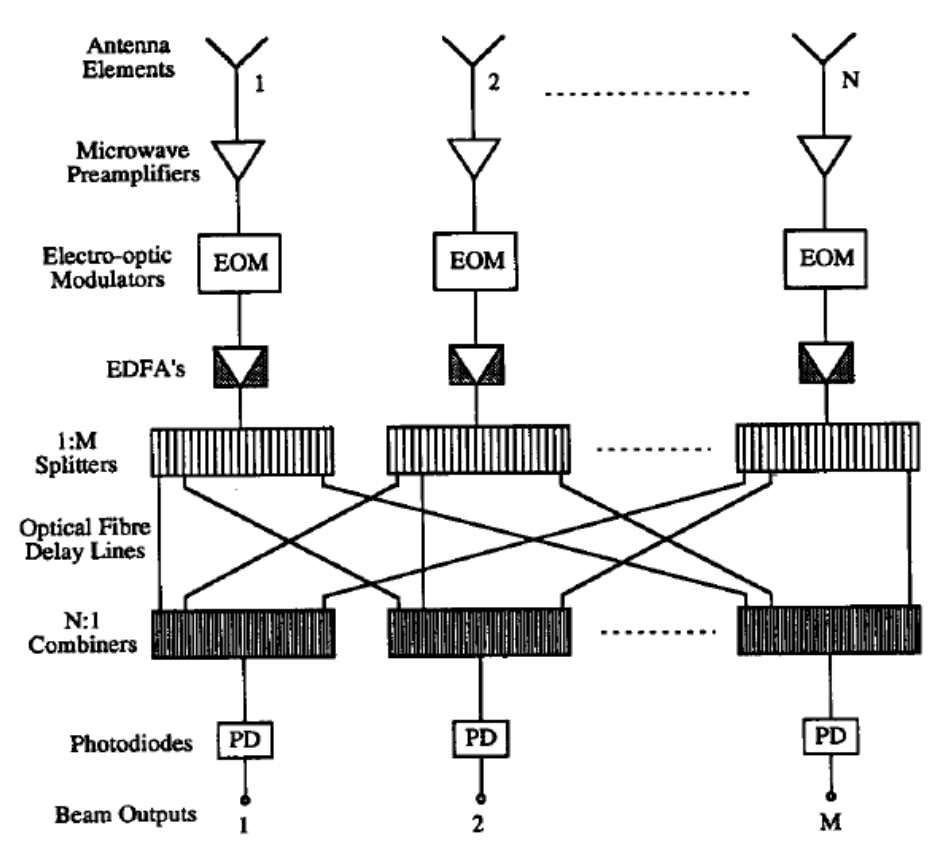

Figure 23. Passive optical TTD architecture for re ceive muti-be am ante nna, bas ed on fibre fibre-optic delay lines [ALA95].

\subsubsection{WDM beamformers based on Dispersion Fibre Prism, Fibre Bragg Gratings and Wavelength Multiplexers}

In 1992, R. Soref proposed a novel optical delay line implementation based on fibre dispersion $\left(\beta_{2}\right)$, since until that time the schemes proposed were based on the use of different optic path lengths in free space or different lengths of optical fibre to achieved the desired time delay (Figure $24 ;$ Error! No se encuentra el origen de la referencia.). The aim of this proposal was to provide reduce hardware and complexity by the parallelization of the time delay. [SOR92]. However, this approach requires as many lasers as antenna elements in the phased antenna array.

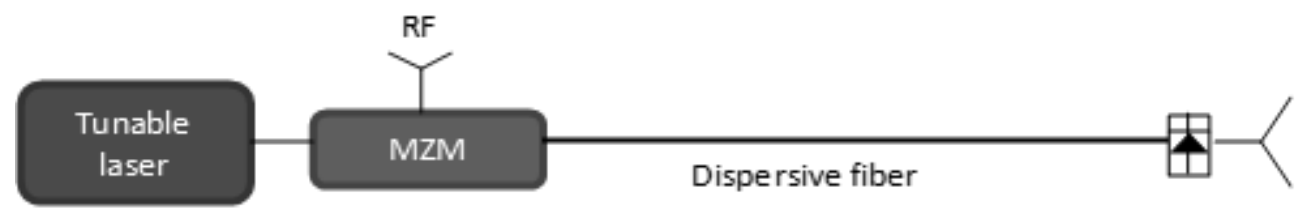

Figure 24. B lock diagram of the optical delay line architecture based on fibre dispersion [SOR92]. 
In order to further reduce the hardware complexity in photonic beamformers, and/or to form multiple beams simultaneously, wavelength division multiplexing (WDM) has been extensively explored [TON96], [VID12].

The concept of the dispersion prism beamformer shown in Figure 25 was proposed by R. Esman and co-workers [ESM93] [ESM95]. The fibre prism is made of an appropriate combination of lengths of high and low dispersion fibres. For a centre wavelength, the time delays in all channels are equal. For longer (shorter) wavelengths, high dispersion fibres add (subtract) time delay, resulting in different antenna element phasing. Typical dispersion of commercial fibres is $80 \mathrm{ps} /(\mathrm{km} \mathrm{nm})$ - optical signals with a wavelength separation of $1 \mathrm{~nm}$ acquire 8 ps differential delay after $1 \mathrm{~km}$. Much higher dispersion fibres have been achieved, and in particular by using photonic crystal fibre (PCF), which could reduce, by one order of magnitude or two, the required fibre lengths [JIA05] [CHE08].

It can be applied to fixed MBA beamformer by using multiple channels with fixed-wavelength WDM laser sources, or to beam-hopping by using wavelength-switched lasers. In [ESM98], the concept of dispersion fibre prism was spread on the two-dimensional phased-array antenna, operating in transmit and receive modes.

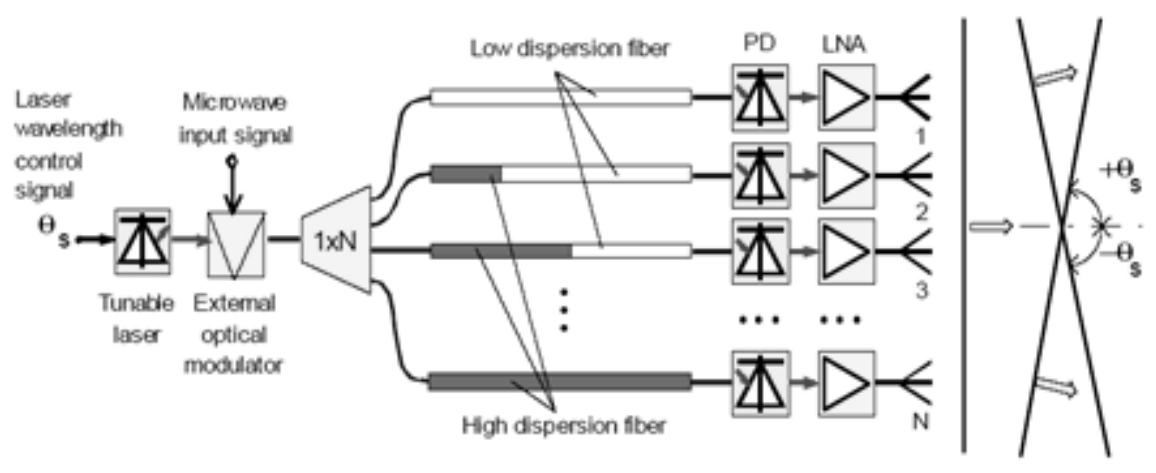

Figure 25. 2-D mode beamformer for trans mit array ante nna base d on Es man's dis persion fibre prism.

Fibre Bragg grating (FBG) filters are band-pass $(0.1-10 \mathrm{~nm})$ reflective filters that can be used in WDM TTD architectures. Figure 26 shows such a FBG-based photonic beamformer architecture where continuously variable TTD is achieved by employing tuneable lasers and one wide bandwidth chirped-fibre grating as dispersive element [COR97]. 


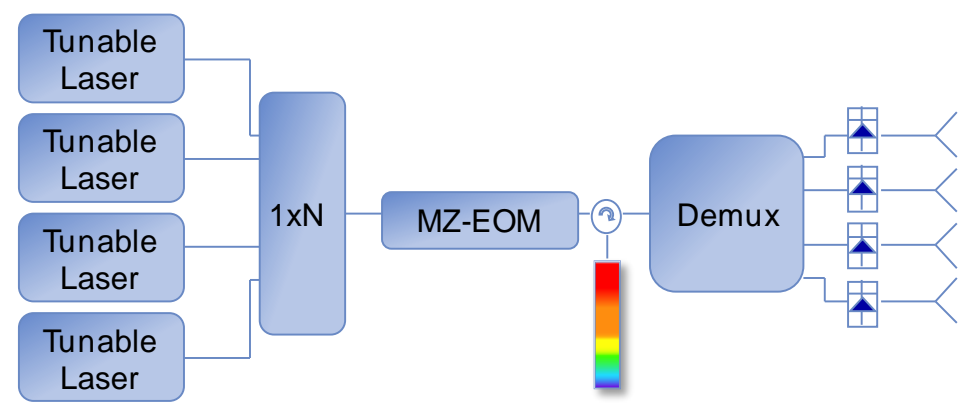

Figure 26. FB G-based photonic be amformer archite cture.

Alternatively, the architecture proposed in [MIN99] is capable of creating multiple simultaneous beams from a receive array antenna. Electro-optical modulators receiving RF signals from the antenna elements are fed by equally-spaced wavelengths, and connected through a star coupler providing outputs for $M$-independent beams. The delay profiles required to achieve constructive summation at the beam port are created by chirped Bragg gratings [ORT00] for small delay times $(<10 \mathrm{ps})$, and/or discrete Bragg gratings for longer delay times (>10 ps). This FBG-based BF results in a minimum number of interconnects.

In 2002, an approach was reported based on the dispersion prism beamformer concept which consists of a single-mode fibre delay line, a chirped grating delay line and three discrete FBG delay lines [LIU02]. This approach (Figure 28) avoids the need to provide a tuneable multi-wavelength laser source with equally increased or decreased wavelength spacing, or need to fabricate chirped fibre gratings with different chirp rate required in traditional fibre grating prisms. 


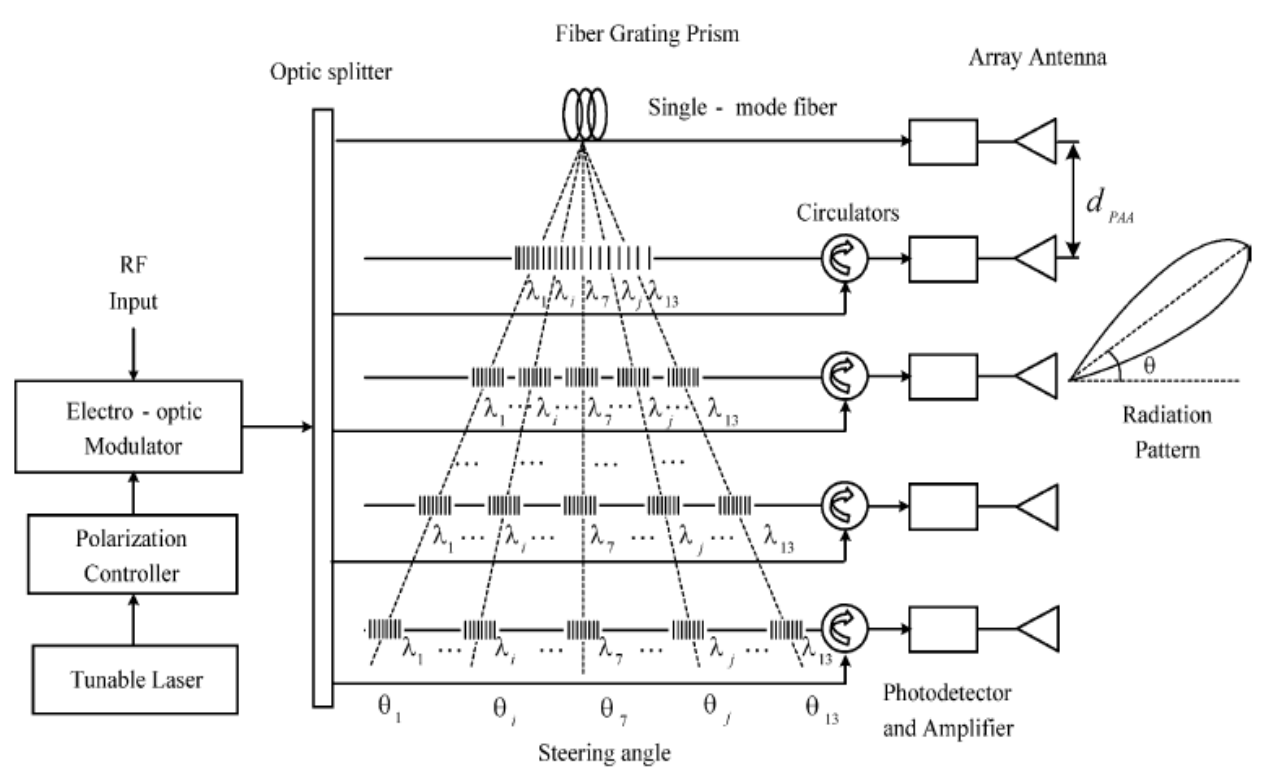

Figure 27. FB G-based, TTD beamformer proposed in [LIU02].

Other WDM photonic TTD architectures have been proposed based on routing through a wavelength-division demultiplexer [YEG00] [RAZ05], either in arrayed waveguide grating (AWG) or thin-film filter technology. Figure 28 shows such a beamformer architecture where the beam light modulated by the input RF signal is send to a circulator and depending on its wavelength, is routed to a different output port, where a particular length of fibre is placed. Next, the beam bounces in a fibre mirror and goes back to the circulator which routes it to the photoreceiver. In 2010, the same scheme has been proposed to implement a photonic beamformer in receive configuration with multibeam capability [YAR10].

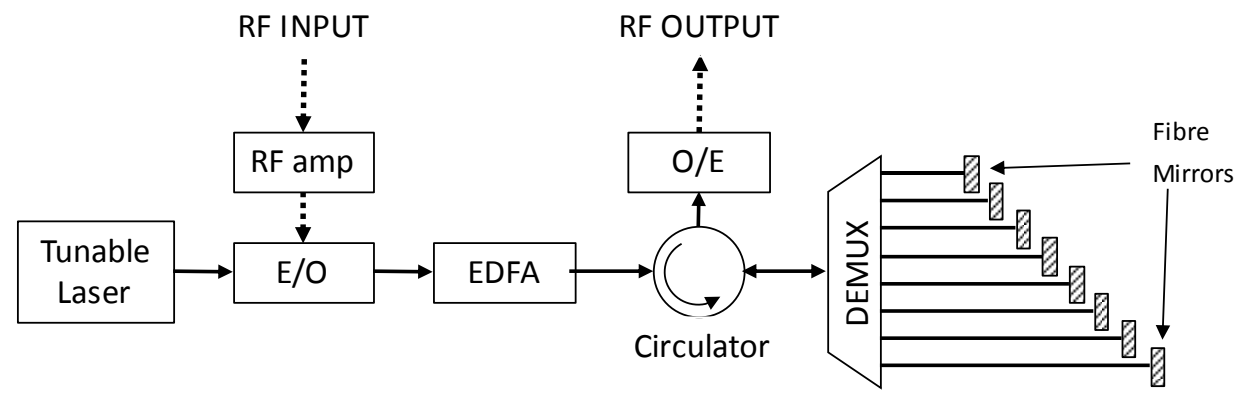

Figure 28. Optical be amformer bas ed on a wave length-division demultiplexer in transmit configuration [RAZ05]. 
In Figure 29, an architecture based on the spectral periodicity of arrayed waveguide gratings (AWGs) and dispersive fibre is depicted [VID03]. This architecture uses multiple sets of wavelengths with a spectral spacing among the signals on each set equal to the free spectral range of the AWG in such a way that all the wavelengths of each set are routed to the same AWG port and pass through the same fibre length in order to obtain multiple simultaneous delays. A wavelength-to-antenna element correspondence is established so fibre dispersion will introduce an equal delay amongst signals of the array elements. This architecture presents straightforward multibeam capability and integration potential to further reduce the complexity and size of the implementation.

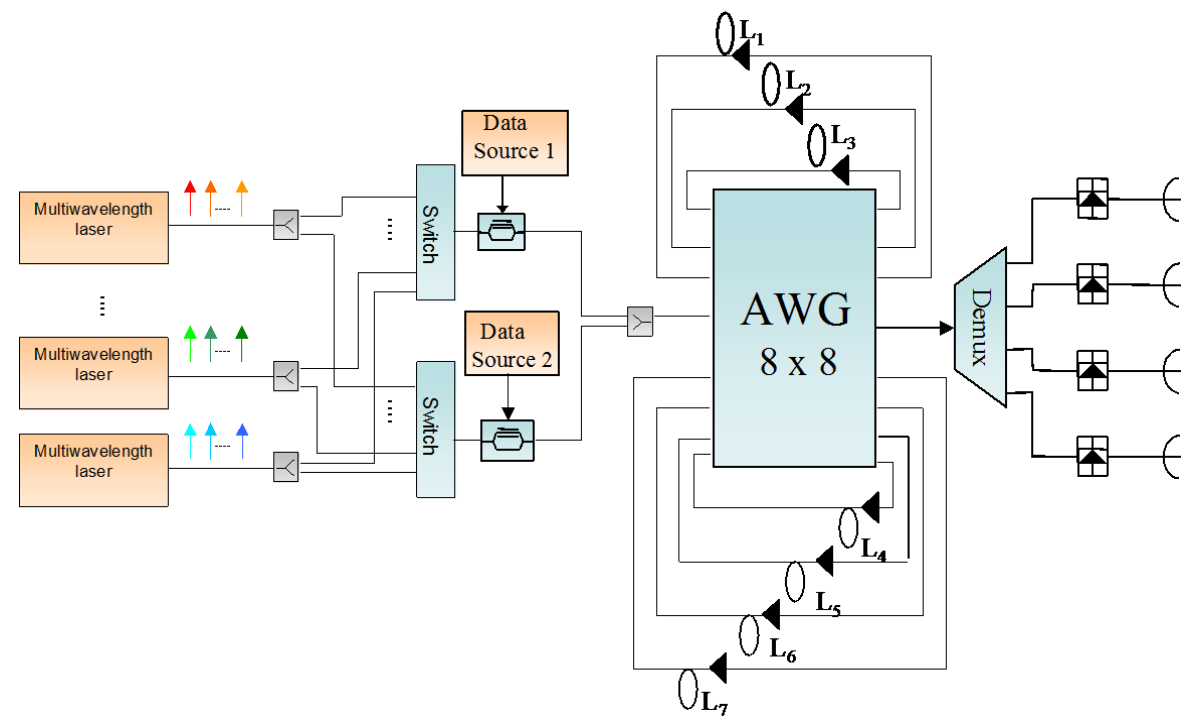

Figure 29. B eamformer based on the spectral pe riodicity of an arrayed wave guide grating and dis persive fibre.

While FBG-based implementations may introduce amplitude/phase distortions or multi-path crosstalk, wavelength-division demultiplexers using AWG or thin film technology can pass the RF modulated optical carriers with no impairments.

The BIFODEL concept was generalized by using high-dispersion delay units, so as to create so-called multi-wavelength programmable dispersion matrices (MPDM) [TON96b]. The dispersive elements can be made of any wavelength-dependent time-delay component. By programming the optical switches, the total dispersion of the MPDM can vary in small increments. In contrast to the conventional switched FODLs, the MPDM creates wavelength-dependent relative time delays among wavelength channels. 
Finally, WDM photonic beamforming can be combined with wavelength-independent TTD concepts, and 2D WDM BF systems have thus been proposed [JUN09], with reduced complexity where the wavelength-dependent TTD controls the azimuth steering; and the wavelength-independent TTD controls the elevation pointing.

\subsubsection{Integrated-optics delay line beamformers}

Integrated optics TTD devices can be realised on a variety of substrates ${ }^{5}$. Planar Lightwave Circuits (PLC) with silica-based waveguides have the advantages of low loss $(<0.1 \mathrm{~dB} / \mathrm{cm})$, and easier polarization maintenance. In [HOR95], an integrated TTD unit having thermos-optic switches and time delay lines was proposed. It was tested around $2.5 \mathrm{GHz}$, and showed phase and amplitude errors respectively less than 0.6 degrees and $0.5 \mathrm{~dB}$.

A 4-bit monolithically integrated TTD device was also demonstrated in [HOW07], on polymer PLC composed of low-loss waveguide delay lines and five $2 \times 2$ thermo-optic switches. The 16 time delays ranging from 0 to $177 \mathrm{ps}$ in $11.8 \mathrm{ps}$ increments were found to be within less than 0.25 ps deviation from the target delays. However, the packaged PLC has insertion loss of up to $14.9 \mathrm{~dB}$ and the switching speed was $2 \mathrm{~ms}$.

Optical integrated TTD circuits for phased-array antennas have also been proposed based on micro-ring resonators [ZHU06], and realized in CMOS compatible, planar optical waveguide technology. A 1x8 chip has been realized as shown in Figure 30. Increasing delays at the different outputs were demonstrated with a maximum of $1.2 \mathrm{~ns}$, a bandwidth of $2.5 \mathrm{GHz}$, and a ripple of $0.1 \mathrm{~ns}$. However, these devices require a number of tunings, and feature high excess loss (about $12 \mathrm{~dB}$ ), high power consumption (approximately $8 \mathrm{~W}$ ) and large switching time.

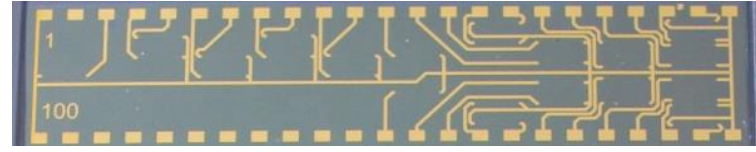

Chip size is $4.85 \times 0.95 \mathrm{~cm}$

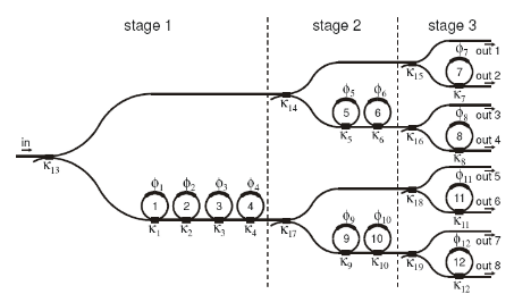

Figure 30. Inte grated, $1 \times 8$ optical TTD de vice based of 12 optical ring resonators [ZHU06].

${ }^{5}$ See Section 1.2. 
In addition, this solution suffers a trade-off between maximum achievable delay, operating frequency and bandwidth, since the linear response of the optical delay line depends strongly on the absolute frequency of the RF signal. To overcome this issue, in [BUR11] a new approach based on ring resonators has been proposed to implement a reconfigurable optical delay line with separate carrier tuning. With this scheme, the delay bandwidth of the optical delay line is independent of the absolute RF frequency.

Another scheme has been recently present in [MOR12] where an optical tuneable delay line based on a side-coupled integrated spaced sequence of resonator (SCISSOR) structure in which pairs of resonances are tuned in opposite directions around the signal. This design mitigates the deleterious effects of group delay dispersion and provides both wide bandwidth and continuously tuneable long delays without distortion. Results for a device including 20 microresonators show a maximum tuneable delay of 345 ps without distortion of the optical signal and fast switching speed of $10 \mu \mathrm{s}$ [MOR12].

\subsubsection{Phase-shifting coherent optical beamformers}

Coherent beamforming architectures are mostly based on heterodyne optical sources, whose beating frequency is equal to the microwave signal frequency, and on controlling the relative phase of their frequency compounds in order to control the phase of the microwave signals. Many approaches have been proposed in the last decades, ranging from free space implementations at the very beginning, to hybrid implementations using fibres [THA90], [BIR92], [MAD02] as much as possible to convey the signals and gain better stability. Such options are briefly reviewed hereafter.

Figure 31 shows an example of this kind of architecture implemented with optical fibres, where both in phase optical carriers are split in different paths. One optical carries goes directly towards the photodiodes whereas a time delay it is induced in the other carrier. Finally, both carriers are combined and send to the photodiode. 


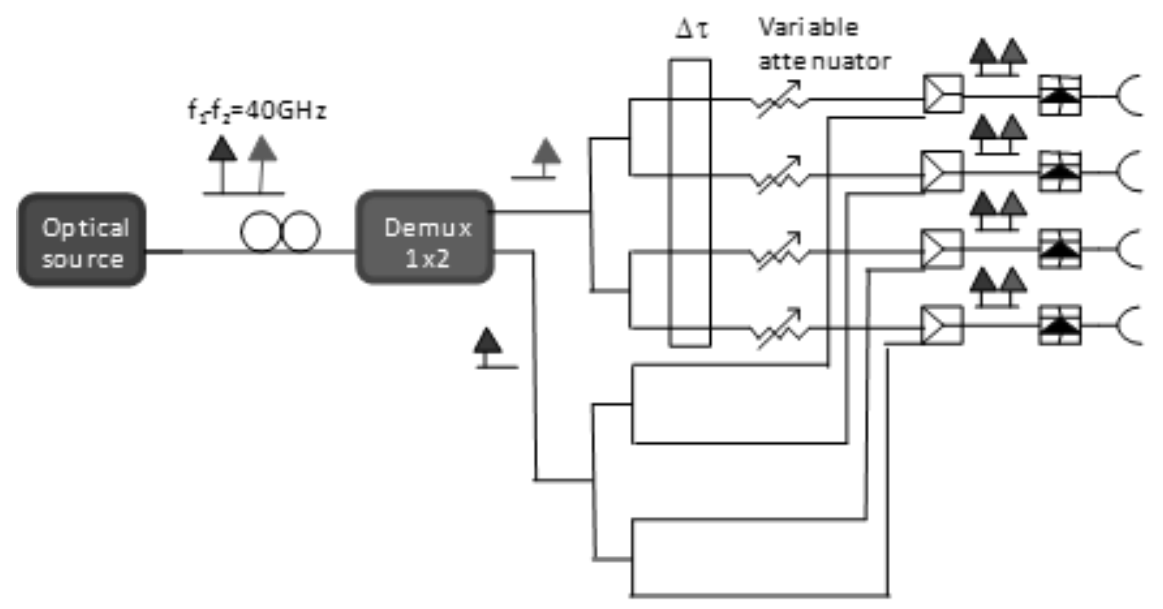

Figure 31. Generic heterodyne optical beamformer architecture implemented with optical fibres.

\subsubsection{Free-space Fourier transform optical signal processors (OSP)}

Another approach is based on the 3D spatial Fourier transform (FT) function of optical lenses and the relationship between the front and rear focal planes, as proposed by [KOE86]. In [AKI01] for example, the spatial light amplitude distribution in the front focal plane is converted by optical FT to a phase distribution in the back focal plane. Sampling in the backfocal plane can be achieved by an array of micro-lenses or a bundle of fibres that support connection to the remote elements of the array antenna.

The major advantage of this approach is its potential for compactness and simplicity. However, the fabrication of the 3D optical fibre array with the required accuracy is very critical, not yet suitable for mass production, and makes it difficult to master the relationship between the light and the microwave beam direction. In order to miniaturize and mechanically stabilize the assembly, and to reduce the optical alignment difficulties and lower optical losses, [AKI01] has developed a 3D OSP-based OBFN, where sampling was achieved through multilayer polymeric optical waveguide arrays, but still significant insertion losses were obtained.

\subsubsection{Hybrid SLM-based coherent beamformers}

It is possible to build hybrid architectures to reduce hardware. For example, in the beamformer proposed by [VID06]-[JOF08] provides propagation TTD to subarrays and phase control to each element of the subarray by SLMs. Thus, the parallelism of SLMs and free-space optics is exploited for phase shifting while implementing TTD with fibre optical delay lines (ODL) to avoid as much as possible 
collimation and loss issues and to improve scalability. In particular, a nematic parallel-alignment SLM (PAL-SLM) was used to control the phase of the RF signal at each antenna element. Effective control of a large number of antenna elements based on a single PAL-SLM was successfully proven. However, the scalability of the demonstrator to larger scales was considered to be limited by the need of collimated beams and by diffraction issues.

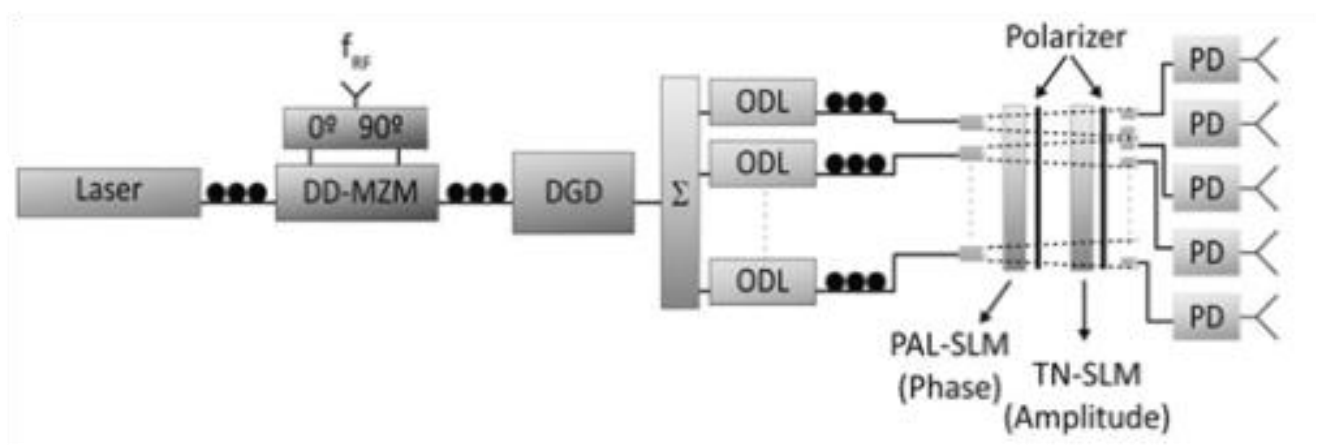

Figure 32. Sche matic diagram of an optical be amformer with TTD and phase and amplitude control [VID06].

Another approach is to use WDM in SLM-based coherent architectures. In [AKI09], a multiple-beam heterodyne beamformer using spatial- and wavelengthdivision multiplexing was proposed. It consists of WDM electro-optic (E/O) converters, generating both signal and LO lightwave compounds for each beam, an SLM-based optical beamforming (OBF) unit, and opto-electronic $(\mathrm{O} / \mathrm{E})$ converters connecting to the array antenna radiating elements. The WDM beam signal compounds are separated spatially by a wavelength filter and illuminate a separate portion of a phase-only spatial light modulator (PSLM) array. Each beam signal compound is thus phase-modulated separately, and then recombined with its LO compound before coupling to the $2 \mathrm{D}$ fibre array connecting to the antenna array.

\subsubsection{Optical coherent beamformer based on integrated phase-shifters}

A self-heterodyning optical waveguide beamforming and beam steering network integrated on electro-optic Lithium Niobate substrate (LN-BFN) was reported in [HOR95]. It contained an optical frequency shifter and a $2 \times 8$ optical signal processing circuit (OSPC), which included optical phase shifters and attenuators. However, the OSPC chip was as large as $68 \times 3 \mathrm{~mm}$, and the optical insertion loss was $17+0.5 \mathrm{~dB}$. 
Optical beamforming devices have been integrated on Indium Phosphide [STU99] [VLI99] and Silica PLC [GRO02] substrates. In [GRO02], an 8-element $\mathrm{BF}$ device was achieved, by using thermo-optic (10 ms response time) actuators to control the amplitude and phase of optically-generated $60 \mathrm{GHz}$ signals. [STU99] [VLI99] have been using InP technology for the integration of a double 1x16 distribution device with fast phase shifting and attenuation control sections, within small dimensions $(8.5 \times 8 \mathrm{~mm})$, but with on-chip losses as high as $28 \mathrm{~dB}$, and extra coupling losses around $5 \mathrm{~dB}$.

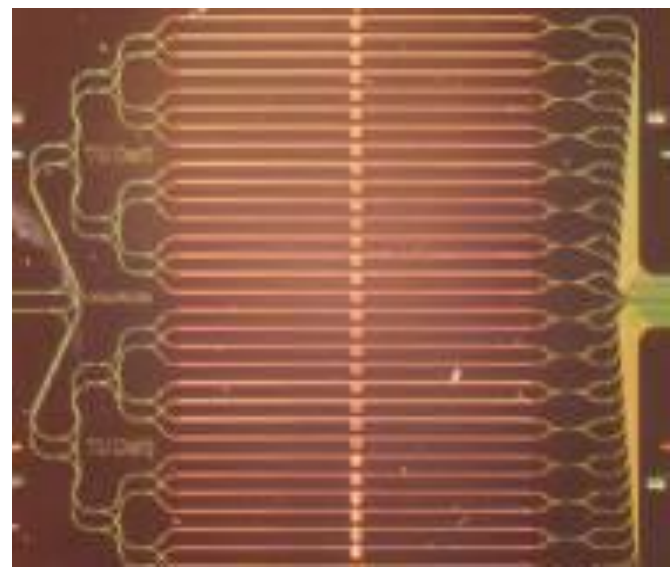

Figure 33. Inte grated, 16-channel InP beamforme r chip [VLI99]. 


\subsection{Conclusion}

The main optical BF architectures and their general advantages and disadvantages are reviewed in Table 3. In general, the practical implementation of a beamforming network will depend on the required performance as well as on the physical and environmental needs. For example, in systems requiring a single beam or a low number of beams the architectures based on switched paths in both fibre or integrated propagation media are the best solution. In this case, the architectures based on the fibre prism like the Esman prism or on the Rotman lens are of special interest since these are passive structures very robust in harsh environments like in military platforms. Nevertheless, the use of OBFN in practical systems requiring a high number of antenna elements and simultaneous beams are limited fundamentally by the fact that the high parallel configurations are complex and difficult to stabilize (as the SLM based solutions) or requires a very bulky implementation, which is a limiting factor in platforms that requires low mass, size and power consumption like satellites and airplanes. Finally, there is a common handicap in all the cases which is the inherent losses and induced noise of the electrical-to-optical conversions. The photonic solutions should offer practical advantages over the RF implementations that justify its use even considering the optoelectronic losses, which is the case for ultra-broadband applications requiring true time-delay beamforming or with integrated solutions that addresses the need of low mass and size and also could benefit from the advantages of the optical fibre to implement centralized optical beamformers and distribute the signal to the antenna elements, reducing the mass and complexity of the antenna itself (in RF solutions the typical configuration is to integrate the beamforming network close to the antenna and the associated amplification in order to reduce the ohmic losses of the RF cables and waveguides). 
TABLE 3: COMPARISON OF MAIN OPTICAL BEAMFORMING CONCEPTS AND TECHNOLOGIES

\begin{tabular}{|c|c|c|c|}
\hline CLASS & $\begin{array}{l}\text { CONCEPT / } \\
\text { TECHNOLOGIES }\end{array}$ & ADVANTAGES & DISADVANTAGES \\
\hline \multirow{4}{*}{$\begin{array}{l}\text { True time } \\
\text { delay optical } \\
\text { beamformers }\end{array}$} & $\begin{array}{l}\text { Based on Free-Space optical } \\
\text { delay lines } \\
\text { [DOL95][RAD03] }\end{array}$ & $\begin{array}{l}\text { High parallel processing power } \\
\text { Multi-beam capability }\end{array}$ & $\begin{array}{l}\text { Significant insertion loss } \\
\text { Stability vs environmenta } \\
\text { conditions } \\
\text { Significant volume } \\
\text { Require fast SLM for beam- } \\
\text { hopping }\end{array}$ \\
\hline & $\begin{array}{l}\text { Based on semi-guided Blass } \\
\text { Matrix [BLA60] [CHE02], or } \\
\text { Rotman lens [CUR95] } \\
\text { [ZAL09]. }\end{array}$ & $\begin{array}{l}\text { Multi-beam capability } \\
\text { Wide bandwidth beamformer } \\
\text { Compact BF }\end{array}$ & $\begin{array}{l}\text { Significant insertion loss } \\
\text { Limited scalability } \\
\text { Limited resolution } \\
\text { Requires fast switches for beam- } \\
\text { hopping }\end{array}$ \\
\hline & $\begin{array}{l}\text { Based on switched fibre } \\
\text { optical delay lines (FODEL) } \\
\text { [SOR84] [MAD10] }\end{array}$ & $\begin{array}{l}\text { High flexibility } \\
\text { Limited complexity } \\
\text { Wavelength independent } \\
\text { Beam-hopping capability }\end{array}$ & $\begin{array}{l}\text { Limited scalability } \\
\text { Bulky implementation } \\
\text { Require fast switches for beam- } \\
\text { hopping }\end{array}$ \\
\hline & $\begin{array}{l}\text { Based on fibre optic Rotman } \\
\text { lens [ALA95] [SPA98] }\end{array}$ & $\begin{array}{l}\text { Multi-beam capability } \\
\text { Low complexity } \\
\text { Wavelength independent }\end{array}$ & $\begin{array}{l}\text { Limited scalability } \\
\text { Bulky implementation } \\
\text { Require fast switches for beam- } \\
\text { hopping }\end{array}$ \\
\hline
\end{tabular}




\begin{tabular}{|c|c|c|c|}
\hline CLASS & $\begin{array}{l}\text { CONCEPT / } \\
\text { TECHNOLOGIES }\end{array}$ & ADVANTAGES & DISADVANTAGES \\
\hline & $\begin{array}{l}\text { Based on Optical Ring } \\
\text { Resonators integrated on SoI } \\
\text { substrate [ZHU06] [BUR11] } \\
\text { [MOR12] }\end{array}$ & Integrated device & $\begin{array}{l}\text { Trade-off between bandwidth and } \\
\text { delay } \\
\text { High delay ripple } \\
\text { Significant insertion losses } \\
\text { Wavelength dependent } \\
\text { May require many tunings }\end{array}$ \\
\hline & $\begin{array}{l}\text { Esman fibre Prism based on } \\
\text { high/low dipersion fibres, and } \\
\text { WDM lasers [ESM93] } \\
\text { [ESM95][JIA05] [CHE08] }\end{array}$ & $\begin{array}{l}\text { Wide bandwidth beamformer } \\
\text { High flexibility } \\
\text { Low insertion loss } \\
\text { Multi-beam capability at low complexity } \\
\text { Beam-hopping with switchable- } \lambda \text { laser } \\
\text { Limited \# of optical interconnects }\end{array}$ & $\begin{array}{l}\text { May require long fibre lengths } \\
\text { Significant volume } \\
\text { Thermal sensitivity }\end{array}$ \\
\hline & $\begin{array}{l}\text { WDM BF based on Fibre } \\
\text { Bragg Gratings, or AWG } \\
\text { (Arrayed } \\
\text { Grating) devices, and WDM } \\
\text { lasers [MIN97] [COR97] } \\
\text { [MIN99] [JAL98] [YEG00] } \\
\text { [LIO02] [VID03] [RAZ05] } \\
\text { [YAR10] }\end{array}$ & $\begin{array}{l}\text { Wide band beamformer } \\
\text { High flexibility } \\
\text { Low insertion loss } \\
\text { Multi-beam capability at low complexity } \\
\text { Beam-hopping with switchable- } \lambda \text { laser } \\
\text { Limited \# of optical interconnects }\end{array}$ & $\begin{array}{l}\text { High number of FBG's or AWG's } \\
\text { High manufacturing cost } \\
\text { Multi-path interference for FBG's } \\
\text { Less resolution for AWG's }\end{array}$ \\
\hline
\end{tabular}




\begin{tabular}{|c|c|c|c|}
\hline CLASS & $\begin{array}{l}\text { CONCEPT / } \\
\text { TECHNOLOGIES }\end{array}$ & ADVANTAGES & DISADVANTAGES \\
\hline \multirow{3}{*}{$\begin{array}{l}\text { Phase- } \\
\text { shifting } \\
\text { coherent } \\
\text { optical beam } \\
\text { formers }\end{array}$} & 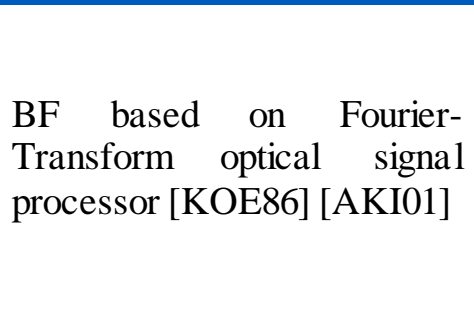 & $\begin{array}{l}\text { High parallelism } \\
\text { Low mass and volume }\end{array}$ & $\begin{array}{l}\text { High insertion losses } \\
\text { Opto-mechanical alignment } \\
\text { issues } \\
\text { Stability vs environmental } \\
\text { conditions } \\
\text { Require fast SLM matrix for } \\
\text { beam-hopping }\end{array}$ \\
\hline & $\begin{array}{l}\text { Hybrid BF based on SLM } \\
\text { (Spatial Light Modulator) } \\
\text { arrays [BLA03] [AKI01] } \\
\text { [JOF08] }\end{array}$ & $\begin{array}{l}\text { High parallelism } \\
\text { Low mass and volume }\end{array}$ & $\begin{array}{l}\text { High insertion losses } \\
\text { Stability vs environmental } \\
\text { conditions } \\
\text { Require fast SLM for beam- } \\
\text { hopping }\end{array}$ \\
\hline & $\begin{array}{l}\text { BF based on integrated } \\
\text { phase-shifters on Lithium } \\
\text { Niobate [HOR95] Silica } \\
\text { [GRO02] InP semiconductor } \\
\text { [VLI99] [STU00] }\end{array}$ & Low mass and volume & $\begin{array}{l}\text { High insertion losses } \\
\text { Limited scalability } \\
\text { Dependence to environmental } \\
\text { conditions } \\
\text { Substantial power consumption }\end{array}$ \\
\hline
\end{tabular}




\section{CHAPTER 3}

\section{Photonic \\ Switched Beamformer for Broadband Wireless Access}

This chapter reports experimental results for a photonic beamformed beam-switched phased-array antenna in transmission (downstream) and reception (upstream) modes intended for high-bandwidth base station (BS) smart antennas in fixed and mobile Broadband Wireless Access (BWA) in the mm-wave band ${ }^{6}$. The application scenario has been defined in order to demonstrate the feasibility of optical beamforming in a test platform with specifications that emulate a realistic scenario in a wireless radio network in the $40 \mathrm{GHz}$ band. This wireless network is based on a cellular pattern and 90 degrees sectors offering services with payload rates up to $155 \mathrm{Mb} / \mathrm{s}$ for fixed and mobile users. The duplexing approach is TDD and the multiple access method is TDMA. In order to improve the radio link performance a space-switched single-beam antenna covering the whole sector from the base station is proposed. This beam-switching approach matches perfectly with a time division access method as a base station antenna is illuminating a user terminal in the desired direction in the assigned time-slot.

6 The work described in this chapter has been published in the publication A, B, C, G, H and I, which were carried out in the frame of the EU project OBANET IST-2000-25390. 
Figure 34 shows a schematic of the envisaged scenario: a single beam is switched between users on a time-slot frame-interval basis. A basic frame structure is depicted in the same figure.

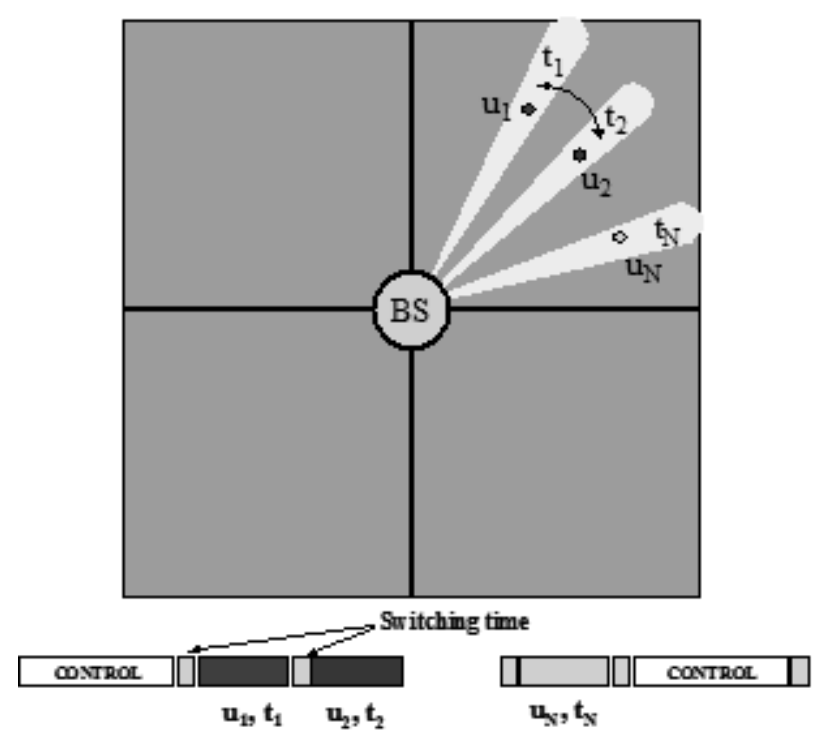

Figure 34. Application scenario.

\subsection{Beamformer Architecture Description}

\subsubsection{Transmission Mode}

The beamforming architecture in the transmission mode is depicted in Figure 35. A set of optical carriers generated by individual laser sources (multi-wavelength laser, MWL) is externally modulated using an electro-optical MZM driven by an mm-wave amplifier. The MZM output is launched into a TTD switching matrix comprised by four cascaded optical switches. Standard single mode fibre (SSMF) coils of lengths $L, 2 L$, and $4 L$, are placed between the upper ports of consecutive switches. Therefore, the optical signal is routed through different aggregated lengths of fibre depending on the bias voltages of the switches, up to eight different possible combinations (3-bit). Due to the wavelength-dependent dispersive behaviour of the optical fibre, each modulated optical carrier suffers a different time delay $\tau_{i}$ when travels through the switching matrix and dispersive fibres, depending on the selected path as given by eq. (1.6). This relative time delay between optical carriers corresponds to different beam steering angles according to eq. (2.11). The WDM optical signal is amplified using an Erbium-doped fibre amplifier (EDFA), then each optical carrier is demultiplexed and launched to a high-speed photo-receiver, where the signal is converted back to the electrical domain. After amplification using an 
mm-wave amplifier, the four modulated electrical carriers are radiated using a $\lambda / 2$ spaced4-element patch antenna. The phase-front of the radiated signal can be varied among eight different positions depending on the chosen combination of switching voltages (i.e. total delays), according to eq. (2.11).

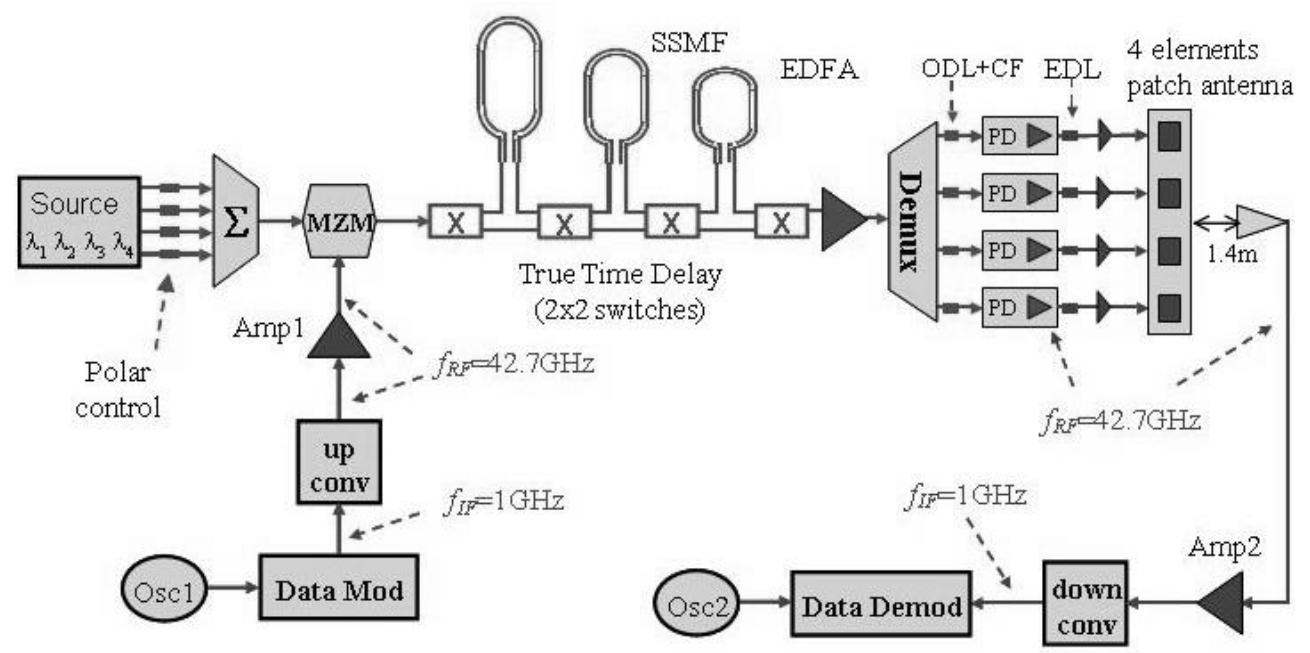

Figure 35. Photonic beam s witched architecture for the trans mission mode.

To avoid the carrier suppression effect, which can result in power degradation in the beamformer performance, single sideband (SSB) modulation can be used. In order to achieve this modulation the optical carriers have been shifted $0.2 \mathrm{~nm}$ from the nominal central band-pass frequencies of the demultiplexer and, in this way, the demultiplexer filters one of the sidebands of each optical carrier.

\subsubsection{Reception Mode}

The schematic of the 3-bit beamformer in reception mode is shown in Figure 36. The 32-QAM modulating signal at $1 \mathrm{GHz}$ was generated using a broadband modem board. This IF signal was up-converted to the millimetre-wave frequency band before being radiated. The received signal at the array antenna elements is translated to the optical domain using as many electro-optical MZM as antenna elements, i.e. four MZMs for a four element antenna. Each amplitude modulated optical carrier is proportionally delayed by the TTD unit which switches between eight different lengths of SSMF. Finally, the four optical modulated carriers are photodetected using a single photodiode. The detected signal is down-converted to IF and demodulated. Figure 37 shows the 3-bit beamformer in receiving mode combined with the control electronics and the array antenna. 


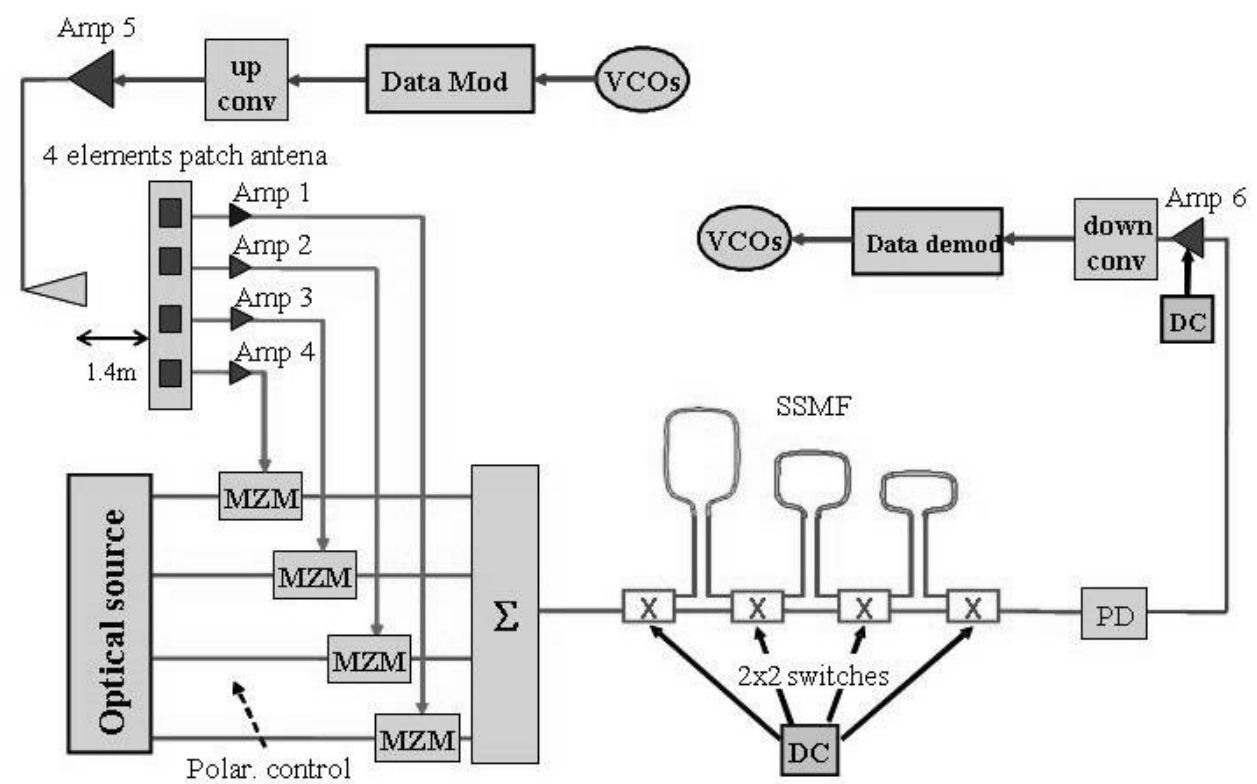

Figure 36. Photonic beam s witched architecture for the reception mode.

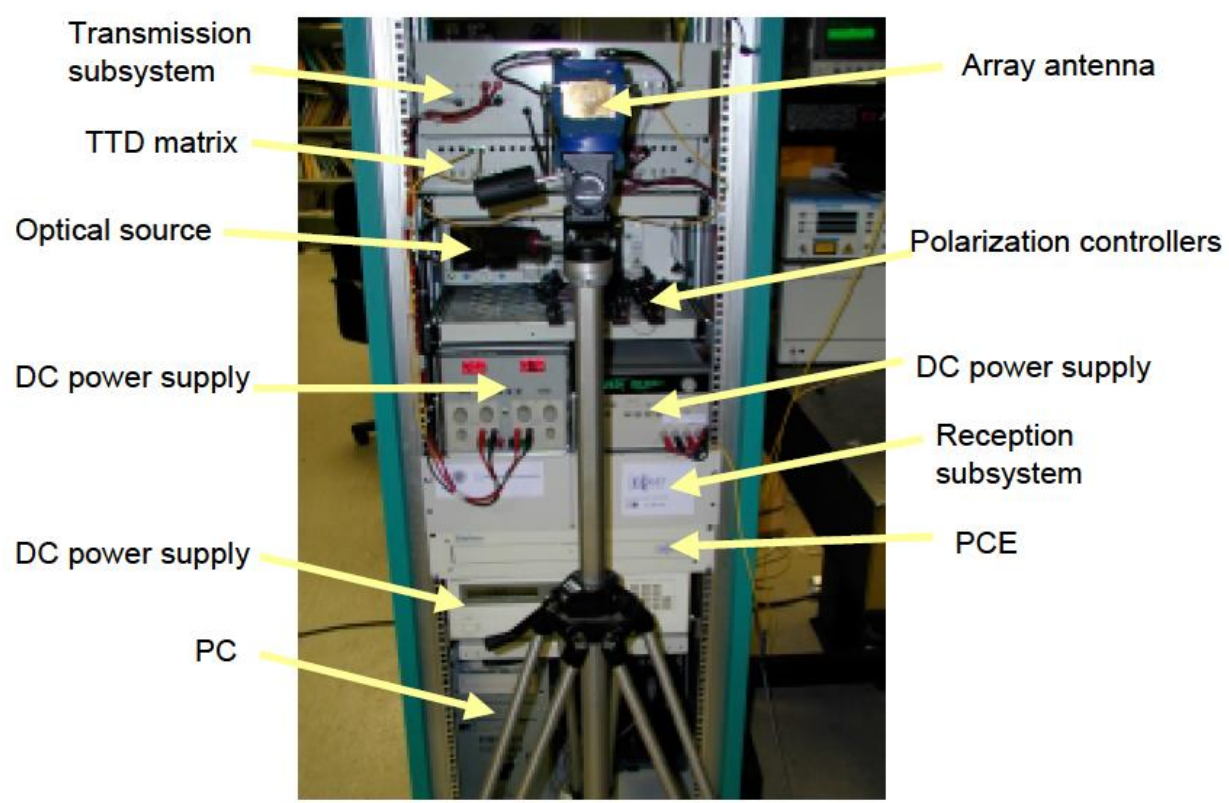

Figure 37. Photonic 3-bit beamformer in re ceiving mode combined with the control electronics and the array antenna. PC: Personal Computer used to control the beamformer. PCE: Photonic Beamformer Control Equipment

(bias source used to bias several components). 


\subsubsection{TTD $2 \times 2$ switching matrix}

The required delays, i.e. the lengths of the fibres, where calculated taking into account the proposed scenario (4-element antenna array and $40 \mathrm{GHz}$ frequency) and the desired beam positions within the 90 degrees sector. The delays depend on the separation of the output wavelengths of the MWL. Table 4 shows the calculated delays and fibre lengths for some combinations of MWL wavelength separation and fibre type. The actual wavelength separation is $\Delta \lambda=3.2 \mathrm{~nm}$ and the selected fibre was Standard SMF.

\section{TABLE 4: REQUIRED FIBRE LENGTHS AS A FUNCTION OF THE WAVELENGTH SEPARATION AND FIBRE TYPE}

$\begin{array}{cccc}\text { Wavelength Separation (fibre type) } & \text { Optical Path } 1 & \text { Optical Path } 2 & \text { Optical Path } 3 \\ \Delta \lambda=3.2 \mathrm{~nm} \text { (Standard SMF) } & 166.60 \mathrm{~m} & 83.30 \mathrm{~m} & 41.65 \mathrm{~m} \\ \Delta \lambda=3.2 \mathrm{~nm} \text { (H. Dispersive SMF) } & 40.46 \mathrm{~m} & 20.23 \mathrm{~m} & 10.11 \mathrm{~m} \\ \Delta \lambda=1 \mathrm{~nm} \text { (Standard SMF) } & 533.10 \mathrm{~m} & 266.55 \mathrm{~m} & 133.28 \mathrm{~m} \\ \Delta \lambda=1 \mathrm{~nm} \text { (H. Dispersive SMF) } & 129.47 \mathrm{~m} & 64.73 \mathrm{~m} & 32.37 \mathrm{~m}\end{array}$

\subsection{Time Delay Calibration}

Both in transmission and reception modes, the length differences between each demultiplexer/multiplexer outputs and the antenna elements have to be compensated to avoid undesired time delay differences among the RF signals. As a first solution, several patch cords of SSMF have been introduced to correct these differences but since the beamformer is intended for the millimetre-wave band, the time delay unbalance between each path has to be lower than $1 \mathrm{ps}$ and therefore the patch cord length accuracy has to be better than $0.2 \mathrm{~mm}$. It is really difficult to obtain such an accuracy in the fibre patch cord length and, therefore, one optical delay line (ODL) has been introduced before the photodiodes in the transmission scheme and after the MZMs in the reception architecture to allow fine adjustment of the time delay. These ODLs can change its absolute time delay up to $300 \mathrm{ps}$ which is equivalent to $6 \mathrm{~cm}$ of SSMF with $n=1.467$.

In this calibration procedure, the first step is to determine the absolute time delay (path length difference) between each branch (from the demultiplexer output to the PD and from the MZM to the multiplexer output). The time delay can be measured using an optical network analyser which can obtain fibre lengths with centimetre accuracy and using a mechanical method for electro-optic devices (external 
modulators and photodiodes). After introducing patch cords to roughly compensate these time delays, the remaining time delay error has to be corrected. Small time delay errors of a few picoseconds appear in these measurements due to noise, accuracy of the VNA (vector network analyser) as well as differences between consecutive connections of the electrical cables. Since the time delay needed for beam-steering is in the order of picoseconds these errors have to be compensated.

Therefore, a complementary procedure has been used to obtain fine time delay calibration. It is based on measuring the radiation pattern of pairs of antenna elements and, by means of the angle position of the radiation nulls, to derive the time delay between them. Three pairs are used: antenna elements 1-2, 2-3 and 3-4. Thus, three antenna arrays with inter-element spacing of $\lambda / 2$ are obtained. Although this fine calibration is based on phase adjusting, thanks to the previous coarse calibration, time delay can be calibrated without ambiguity. Finally, in order to steer the beams to positive and negative angles relative to the centre of the sector (broadside), a fixed time shift of (15.7/2) ps between adjacent elements will be included. Thus, positive and negative steering angles can be obtained as can be seen in Table 5 .

TABLE 5: CORRES PONDENCE AMONG S WITCHING VOLTAGES, OVERALL DELAY AND S TEERING ANGLE WITH RES PECT TO BROADSIDE. (SWITCH CONTROL'0' CORRES PONDS TO CROSS STATEAND ' 1 ' TO BAR STATE).

\begin{tabular}{|c|c|c|}
\hline Switching control & Total time delay $(\mathbf{p s})$ & Stee ring angle (deg) \\
\hline 0110 & 0 & -41.7683 \\
\hline 1010 & 2.2440 & -28.3257 \\
\hline 0000 & 4.4880 & -16.4301 \\
\hline 1100 & 6.7320 & -5.2331 \\
\hline 0101 & 8.9760 & 5.7640 \\
\hline 1001 & 11.2200 & 16.9818 \\
\hline 0011 & 13.4640 & 28.9277 \\
\hline 1111 & 15.7080 & 42.4808 \\
\hline
\end{tabular}

Figure 38 shows the antenna pattern measurement at $42.7 \mathrm{GHz}$ used for the calibration of the time delay between antenna elements 3 and 4 . 


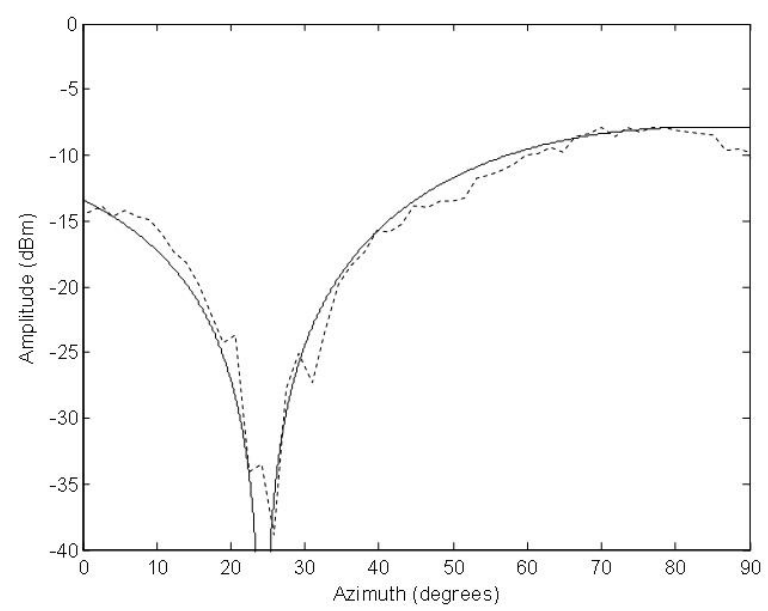

Figure 38. Antenna pattern us ed for calibration of antenna ele ments 3-4. (Solid) theory, (dotted) measured.

\subsection{System Performance Analysis}

The values of the different system parameters of the beamformer are detailed in Table 6. The calculations carried out for evaluating the performance of the designed optical beamformer have been done using the formulas described in the chapter 1 .

TABLE 6: Sys TEM PARAMETERS VALUES USED IN THE PERFORMANCE ES TIMATION S IMULATIONS.

\begin{tabular}{|c|c|c|}
\hline Laser source & Parameter & Value \\
\hline & RIN & $-137 \mathrm{~dB} / \mathrm{Hz}$ \\
\hline & Optical power $\left(\mathrm{P}_{\mathrm{i}}\right)$ & $0 \mathrm{dBm}$ \\
\hline & Wavelength & $1550 \mathrm{~nm}$ \\
\hline \multirow[t]{3}{*}{ MZM } & Parameter & Value \\
\hline & Modulation index (m $\left.\mathrm{m}_{\mathrm{RF}}\right)$ & 0.2 \\
\hline & Insertion Loss & $6 \mathrm{~dB}$ \\
\hline \multirow[t]{2}{*}{ Circulator } & Parameter & Value \\
\hline & Insertion Loss & $1 \mathrm{~dB}$ \\
\hline \multirow[t]{2}{*}{ TTD matrix } & Parameter & Value \\
\hline & Insertion Loss & $30 \mathrm{~dB}$ \\
\hline \multirow[t]{2}{*}{ Demux } & Parameter & Value \\
\hline & Insertion Loss & $5 \mathrm{~dB}$ \\
\hline \multirow[t]{4}{*}{ EDFA $_{1}$} & Parameter & Value \\
\hline & Gain $\left(\mathrm{G}_{1}\right)$ & $20 \mathrm{~dB}$ \\
\hline & Optical bandwidth $(\Delta v)$ & $30 \mathrm{~nm}$ \\
\hline & Noise Figure $\left(\mathrm{NF}_{1}\right)$ & $4.5 \mathrm{~dB}$ \\
\hline
\end{tabular}




\begin{tabular}{|l|l|l|}
\hline EDFA2 & Parameter & Value \\
\hline & Gain $\left(\mathrm{G}_{2}\right)$ & $20 \mathrm{~dB}$ \\
& Optical bandwidth $(\Delta \mathrm{v})$ & $30 \mathrm{~nm}$ \\
& Noise Figure $\left(\mathrm{NF}_{2}\right)$ & $4.5 \mathrm{~dB}$ \\
\hline WGPD & Parameter & Value \\
\hline & Temperature $(\mathrm{T})$ & $300 \mathrm{~K}$ \\
& Responsivity $(\mathfrak{R})$ & $0.7 \mathrm{~A} / \mathrm{W}$ \\
& System bandwidth $(\mathrm{BW})$ & $500 \mathrm{MHz}$ \\
& Dark Current $\left(\mathrm{I}_{\mathrm{d}}\right)$ & $100 \mathrm{nA}$ \\
& Load impedance $\left(\mathrm{R}_{\mathrm{L}}\right)$ & $50 \Omega$ \\
& PD Noise Figure $\left(\mathrm{F}_{\mathrm{n}}\right)$ & $4 \mathrm{~dB}$ \\
\hline
\end{tabular}

\subsubsection{Laser RIN}

If the RIN generated by the MWL increases, the total noise also increases whilst the detected RF signal keeps the same, so the total CNR decreases. Figure 39(left) shows the variation of the system CNR against the laser RIN for several system bandwidths $(250 \mathrm{MHz}, 500 \mathrm{MHz}, 1 \mathrm{GHz}, 2 \mathrm{GHz})$. It can be noticed that the effect of the RIN is not significantly important for values smaller than $-135 \mathrm{~dB} / \mathrm{Hz}$, but for higher values the CNR decreases rapidly. In the region RIN $>-130 \mathrm{~dB} / \mathrm{Hz}$ the dominant noise term is the RIN. It is observed that in this region, CNR decreases $20 \mathrm{~dB} /$ octave, so it is very important to hold the RIN parameter below this limit value.
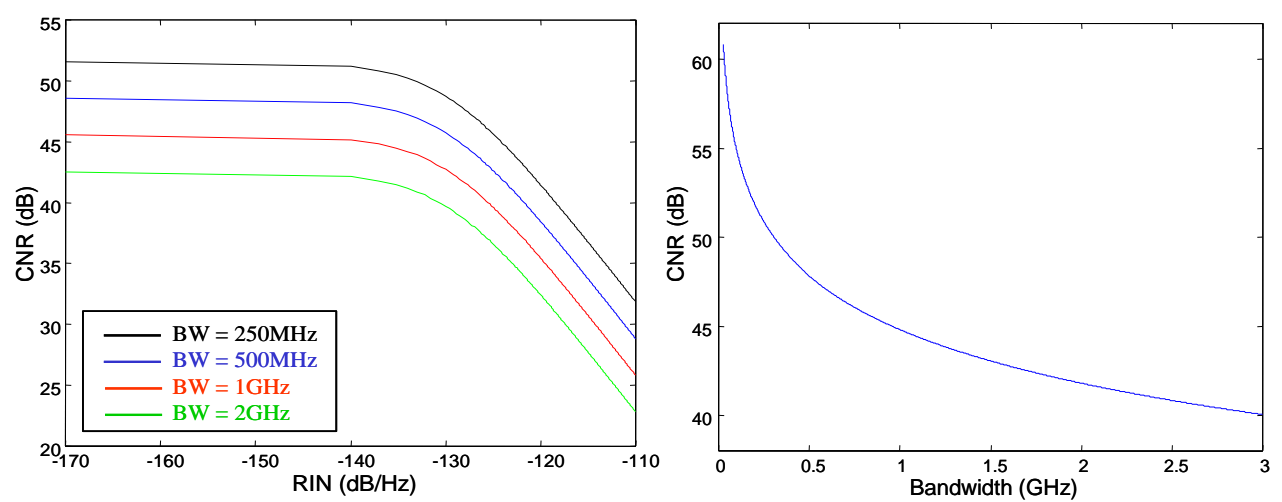

Figure 39. CNR estimation as a function of the las er source RIN for se veral system electrical bandwidths (left) and of the system electrical bandwidth (B W).

\subsubsection{System Electrical Bandwidth}

In these calculations it has been considered that the effective noise bandwidth and the system electrical bandwidth are identical and are defined by a RF filter placed after the photo-receiver. Figure 39(right) shows the CNR estimation as a function of 
the system bandwidth ranging from $25 \mathrm{MHz}$ to $3 \mathrm{GHz}$. It seems very important to achieve a bandwidth smaller than $500 \mathrm{MHz}$ in order to obtain a good CNR. In the system $72 \mathrm{MHz}$ bandwidth is required for $155 \mathrm{Mb} / \mathrm{s}$ QPSK modulated signals and lower bandwidths will be needed for higher level modulations.
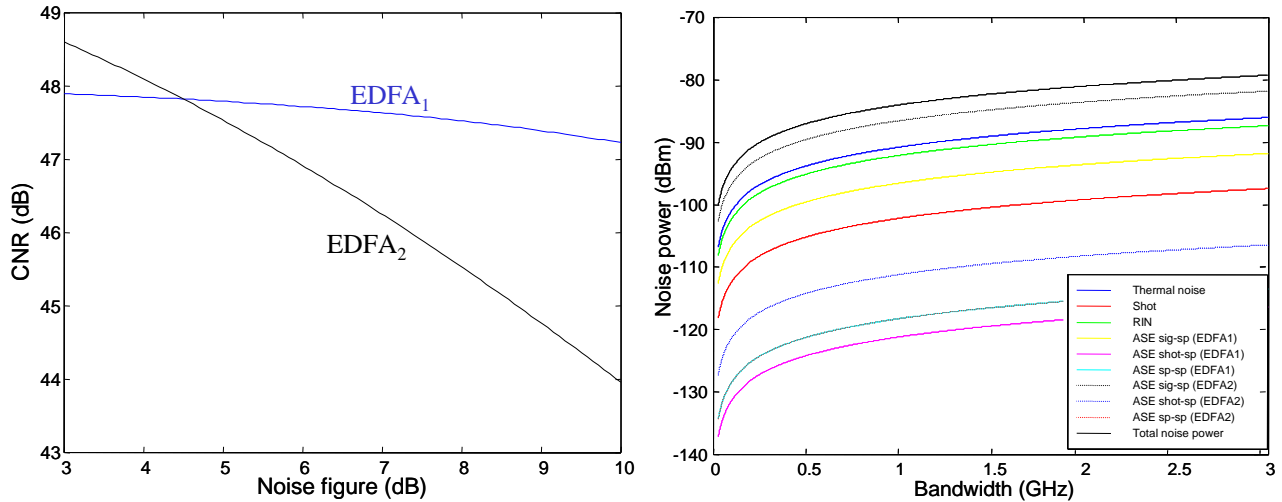

Figure 40. CNR estimation as a function of the noise figure of both EDFAs (left) and Noise power of the different noise terms as a function of the system electrical bandwidth (right).

\section{- Noise figure of the EDFAs}

In the proposed system there are two EDFAs which add ASE noise to the optical signal. Figure 40(left) shows the variation of the estimated CNR as a function of the noise figure of each amplifier. In each case the NF of the other EDFA keeps constant and equal to $4.5 \mathrm{~dB}$ (as observed in the figure, at this value both curves have the same value of $\mathrm{CNR}=47.66 \mathrm{~dB}$ ). It can be noticed that the effect of the secondEDFA is more critical for the system performance. This is due to the smaller signal level at the input of $\mathrm{EDFA}_{2}\left(10 \mathrm{~dB}\right.$ smaller than the level at the input of $\left.\mathrm{EDFA}_{1}\right)$ which originates a larger influence of the ASE noise. It is very important that the second EDFA has a NF as small as possible.

Finally, Figure 40(right) shows all the different noise contributions as a function of the system electrical bandwidth. It can be noticed that the main noise term is the signal-spontaneous beat contribution originated in the second EDFA. The noise power of this term is nearly three times larger than the contribution of the second term, the thermal noise. So, it can be deduced that a proper specification of the second EDFA becomes a fundamental task in order to achieve a good system performance.

\subsubsection{Required Modulation Index}

Figure 41 shows the estimated CNR as a function of the optical modulation index $m_{R F}$ for different system electrical bandwidths. From these results, it is 
straightforward to obtain the required $m_{R F}$ needed to achieve a good performance of the system. The electrical bandwidth considered in this work is $70 \mathrm{MHz}$, so for the parameters stated in Table 6, the obtained CNR is $50.3 \mathrm{~dB}$ and $56.3 \mathrm{~dB}$ for $m_{R F}=0.1$ and 0.2 , respectively. For $m_{R F}=0.2$, the detected $\mathrm{RF}$ power will be $P_{\mathrm{RF}}=-39.23 \mathrm{dBm}$.
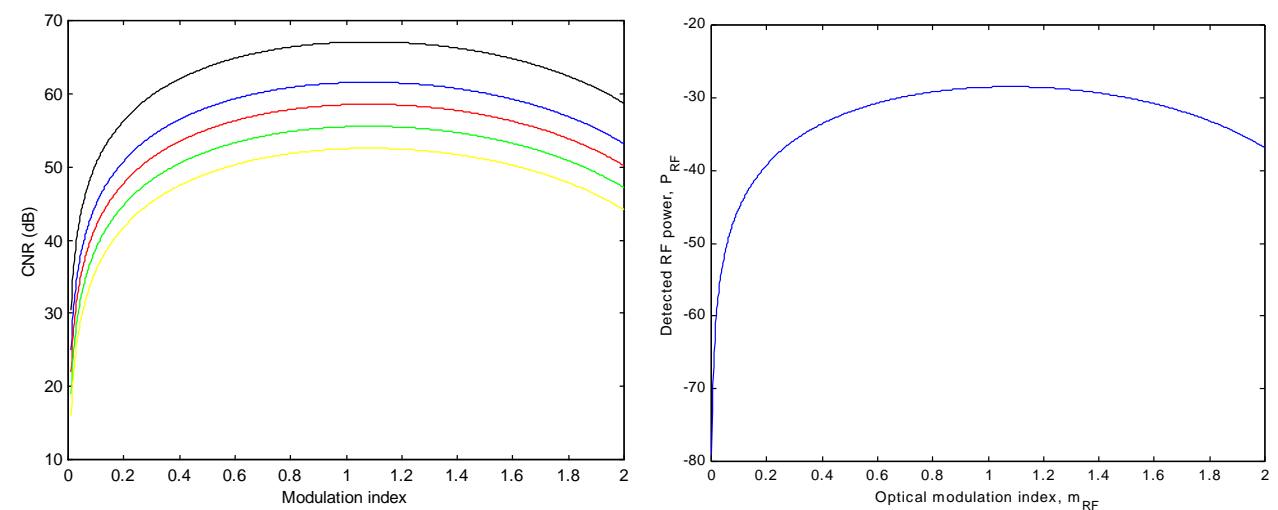

Figure 41. CNR estimation as a function of $m_{R F}$ for diffe rent sys tem e lectrical bandwidths (left) and Detected RF power (fundamental harmonic) vs. optical modulation index, $m_{R F}$ (right).

\subsubsection{1dB Compression Point}

The linear performance margin of the proposed architecture can be obtained from the $1 \mathrm{~dB}$ compression point. Figure 42 shows the output electrical power versus the RF input electrical power at each electrode of a Dual-Drive MZM (from equation (1.23)), considering $\mathrm{V}_{\pi}=10 \mathrm{~V}$ at the working frequency. The RF input power at the electrode of a Single-Drive MZM to obtain the same RF detected power will be $6 \mathrm{~dB}$ higher than the RF input power on each electrode of a Dual-Drive MZM. This figure shows that the $-1 \mathrm{~dB}$ compression point is around $P_{R F-i n}^{D D}=14.7 \mathrm{dBm}$. Nevertheless, the $1 \mathrm{~dB}$ compression point will depend on the $M Z M V_{\pi}$. Figure 42 depicts the $1 \mathrm{~dB}$ compression dependence with $\mathrm{V}_{\pi}$. 

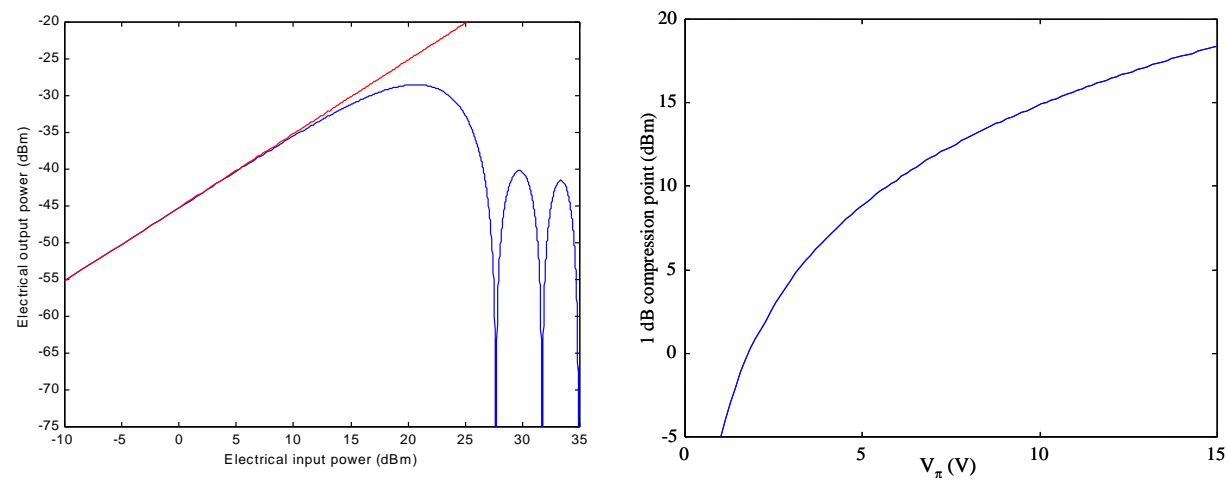

Figure 42. RF detected power versus RF input power showing the $1 \mathrm{~dB}$-compression point (le ft) and $1 \mathrm{~dB}$-compression point versus $\mathbf{V}_{\pi}$ (right).

\subsubsection{Wavelength Stability}

Time delays based on fibre dispersion depend on the separation amongst optical wavelengths $(\Delta \lambda)$ and the fibre length as was described in eq. (1.6). Any variation of this separation will cause a shift of the optical delays. A maximum wavelength excursion of $10 \%$ of the channel spacing is specified for the MWL, where a nominal $3.2 \mathrm{~nm}$ separation has been defined. Some simulations have been carried out to assess this effect. Time delays achieved for the -39.7 degrees beam (worst case) have been shown in Table 7 .

\section{TABLE 7: SimULATION RESULTS OF THE EFFECT OF WAVELENGTH EXCURSION} ON ACHIEVED DELAYS .

\begin{tabular}{|l|c|c|c|}
\hline $\begin{array}{l}\text { Wavelength separation } \\
\text { excursion }\end{array}$ & $\begin{array}{c}\text { Delay between } \\
\text { first and second } \\
\text { elements }\end{array}$ & $\begin{array}{c}\text { Delay between } \\
\text { second and third } \\
\text { elements }\end{array}$ & $\begin{array}{c}\text { Delay between } \\
\text { third and fourth } \\
\text { elements }\end{array}$ \\
\hline Nominal wavelengths & $7.65 \mathrm{ps}$ & $7.64 \mathrm{ps}$ & $7.65 \mathrm{ps}$ \\
\hline $2.5 \%(0.08 \mathrm{~nm})$ & $7.11 \mathrm{ps}$ & $8.11 \mathrm{ps}$ & $7.19 \mathrm{ps}$ \\
\hline $5 \%(0.16 \mathrm{~nm})$ & $6.58 \mathrm{ps}$ & $8.67 \mathrm{ps}$ & $6.67 \mathrm{ps}$ \\
\hline $10 \%(0.32 \mathrm{~nm})$ & $5.19 \mathrm{ps}$ & $10.80 \mathrm{ps}$ & $5.28 \mathrm{ps}$ \\
\hline
\end{tabular}

From the simulation results, the effect of the time delay error on the antenna array pattern can be estimated according with the theory described in Annex A for the residual sidelobe level and the mean gain reduction for a uniform linear array.

If isotropic elements are considered and the array phase errors follow a Gaussian probability distribution with zero mean, the values of Table 8 and Table 9 are obtained. 
TABLE 8: RES IDUAL SECONDARY LOBES RELATIVE TO THE MAIN LOBE.

\begin{tabular}{|c|c|c|c|}
\hline \multirow{2}{*}{ Steering angle } & \multicolumn{3}{|c|}{ Residual secondary lobes relative to the main lobe } \\
\cline { 2 - 4 } & $\begin{array}{c}\text { Wavelength } \\
\text { excursion } 2.5 \%\end{array}$ & $\begin{array}{c}\text { Wavelength } \\
\text { excursion 5\% }\end{array}$ & $\begin{array}{c}\text { Wavelength } \\
\text { excursion 10\% }\end{array}$ \\
\hline $39.7^{\circ}$ & $-\infty d B$ & $-\infty d B$ & $-\infty d B$ \\
\hline $27.22^{\circ}$ & $-35.68 d B$ & $-29.66 d B$ & $-23.64 d B$ \\
\hline $16.04^{\circ}$ & $-29.66 d B$ & $-23.64 d B$ & $-17.62 d B$ \\
\hline $5.45^{\circ}$ & $-26.14 d B$ & $-20.12 d B$ & $-14.01 d B$ \\
\hline$-4.95^{\circ}$ & $-23.64 d B$ & $-17.62 d B$ & $-11.60 d B$ \\
\hline$-15.51^{\circ}$ & $-21.70 d B$ & $-15.68 d B$ & $-9.66 d B$ \\
\hline$-26.66^{\circ}$ & $-20.12 d B$ & $-14.10 d B$ & $-8.08 d B$ \\
\hline$-39.05^{\circ}$ & $-18.78 d B$ & $-12.76 d B$ & $-6.74 d B$ \\
\hline
\end{tabular}

TABLE 9: VARIATION ON THE DIRECTIVITY FOR SEVERAL DIRECTIONS.

\begin{tabular}{|c|c|c|c|}
\hline \multirow{2}{*}{ Steering angle } & \multicolumn{3}{|c|}{$\mathrm{D} / \mathrm{D}_{0}(\mathrm{~dB})$} \\
\cline { 2 - 4 } & $\begin{array}{c}\text { Wavelength } \\
\text { excursion 2.5\% }\end{array}$ & $\begin{array}{c}\text { Wavelength } \\
\text { excursion 5\% }\end{array}$ & $\begin{array}{c}\text { Wavelength } \\
\text { excursion 10\% }\end{array}$ \\
\hline $39.7^{\circ}$ & 0 & 0 & 0 \\
\hline $27.22^{\circ}$ & 0.005 & 0.019 & 0.075 \\
\hline $16.04^{\circ}$ & 0.019 & 0.075 & 0.291 \\
\hline $5.45^{\circ}$ & 0.042 & 0.166 & 0.628 \\
\hline$-4.95^{\circ}$ & 0.075 & 0.291 & 1.061 \\
\hline$-15.51^{\circ}$ & 0.116 & 0.446 & 1.560 \\
\hline$-26.66^{\circ}$ & 0.166 & 0.628 & 2.102 \\
\hline$-39.05^{\circ}$ & 0.224 & 0.835 & 2.666 \\
\hline
\end{tabular}

From values reported in the previous tables, it can be concluded that the wavelength deviations are a critical parameter of photonic beamformers based on WDM and fibre dispersion. For the worst case steering angle (longest path) wavelength excursion will introduce a considerable degradation $(-7 \mathrm{~dB}$ for the residual sidelobe level and $2.7 \mathrm{~dB}$ gain reduction for a $10 \%$ wavelength excursion). Thus, extra care should be taken to bound this excursion in beamformers at millimetre-wave band.

\subsection{Experimental Results}

\subsubsection{Transmission Mode}

The setup used to measure the performance of the beamforming network in transmission mode is depicted in the following figure, where the power budget and 
the spectra of the data signal at the input and the output of the beamformer can be seen.

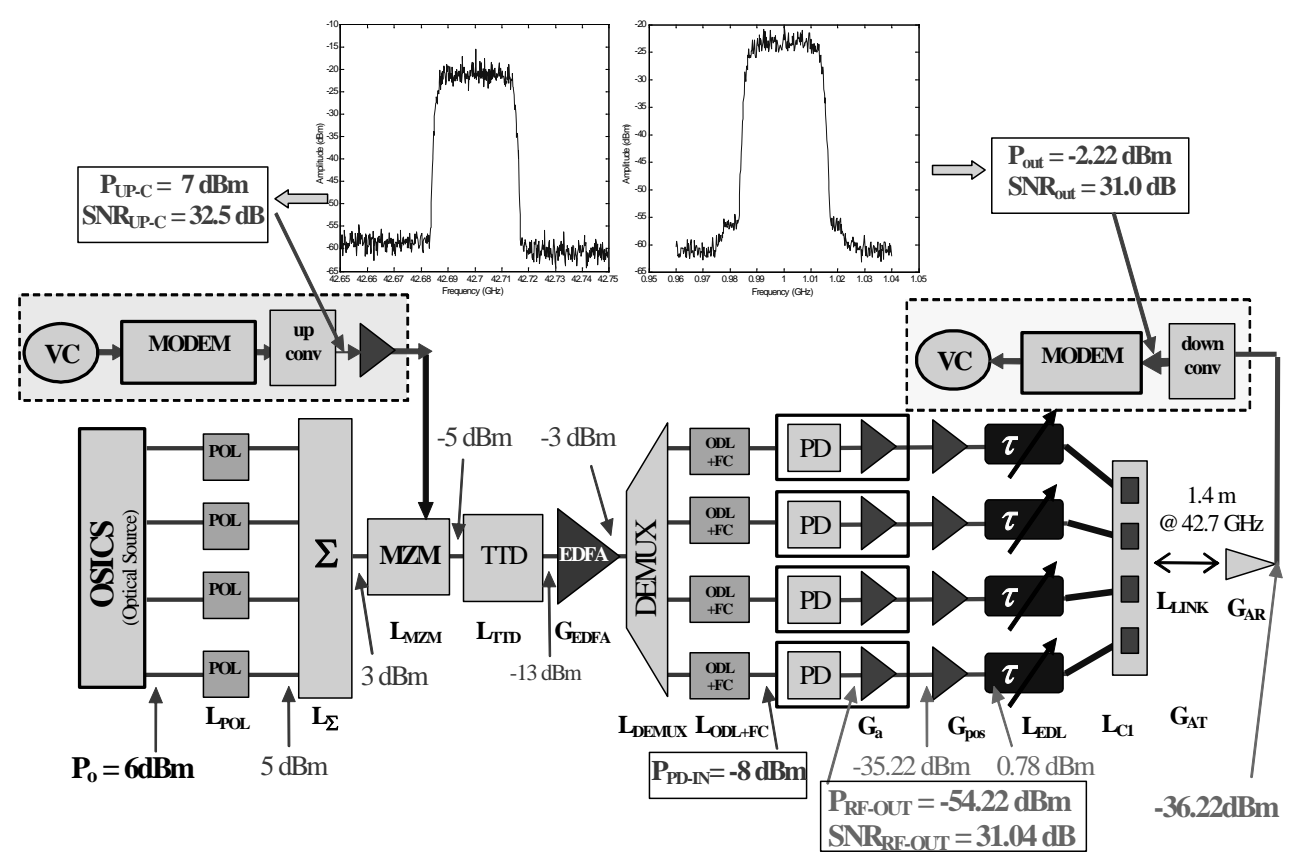

Figure 43. Experimental setup for the trans mis sion mode.

The $155 \mathrm{Mb} / \mathrm{s}$ 32-QAM modulating signal at $1 \mathrm{GHz}$ was generated using one broadband modem board. After filtering, the signal was up-converted to $42.7 \mathrm{GHz}$ using an electrical upconverter. Then, the signal is amplified to obtain a power level of the modulated RF carrier equal to $+7 \mathrm{dBm}$, being its signal to noise ratio (SNR) $32.5 \mathrm{~dB}$. This signal drives the MZM. The modulated WDM signal was launched to the 3-bit TTD switching matrix, controlled by a 4-outputs external tuneable bias source. The lengths of the SSMF fibre coils used to implement the time delays were $170 \mathrm{~m}, 340 \mathrm{~m}$ and $680 \mathrm{~m}$ corresponding to a binary delay line. Before each photoreceiver, an ODL is used for the reasons explained in the previous section. The detected RF signal is further amplified using an mm-wave amplifier with a $36 \mathrm{~dB}$ gain and radiated using the 4-element patch antenna. After propagation through a 1.4 meter radio link (far field at $42.7 \mathrm{GHz}$ ), a $40 \mathrm{~dB}$ gain electrical downconverter, fed by a $40 \mathrm{GHz}$ horn antenna, downconverts the $42.7 \mathrm{GHz}$ signal back to $1 \mathrm{GHz}$. The output SNR is $31 \mathrm{~dB}$ for a signal power of $-2.22 \mathrm{dBm}$ at $1 \mathrm{GHz}$. The intermediate frequency signal is demodulated by a different modem board, which allows to measure signal quality parameters such as the bit error rate (BER). 

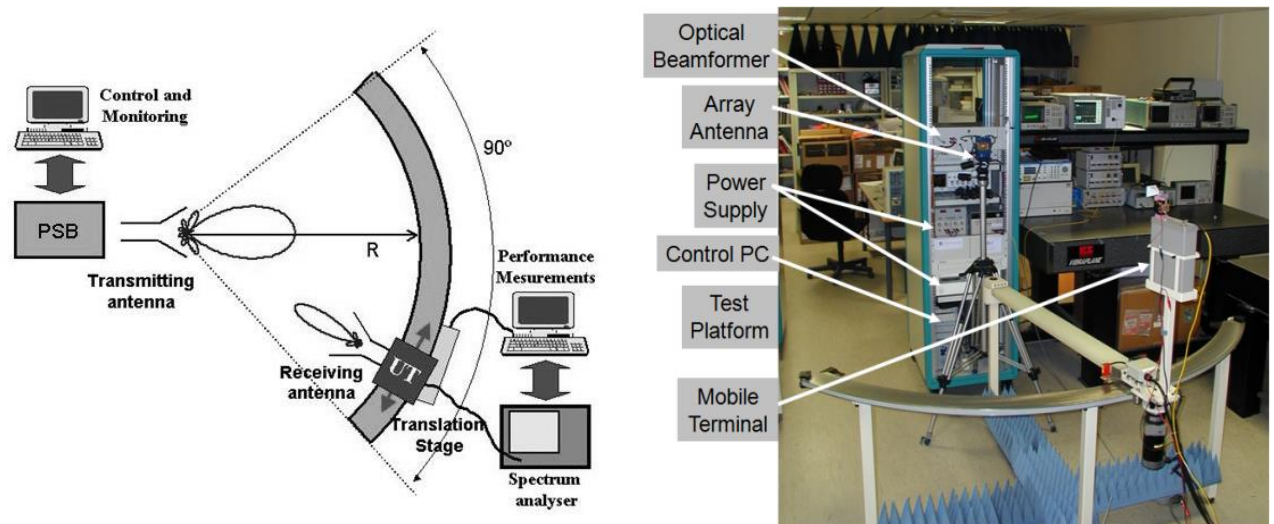

Figure 44. Schematic (left) and photograph (right) of the meas urements set-up us ed to obtain radiation patterns. PSB : Photonic Switched Beamformer.

Figure 44 shows the schematic of the computer controlled measurement platform used to obtain radiation patterns. The downconverter is mounted in a post that can be moved along a 90 degrees circumference arch using a computer controlled step-engine, with programmable speed, acceleration and angular step-size.

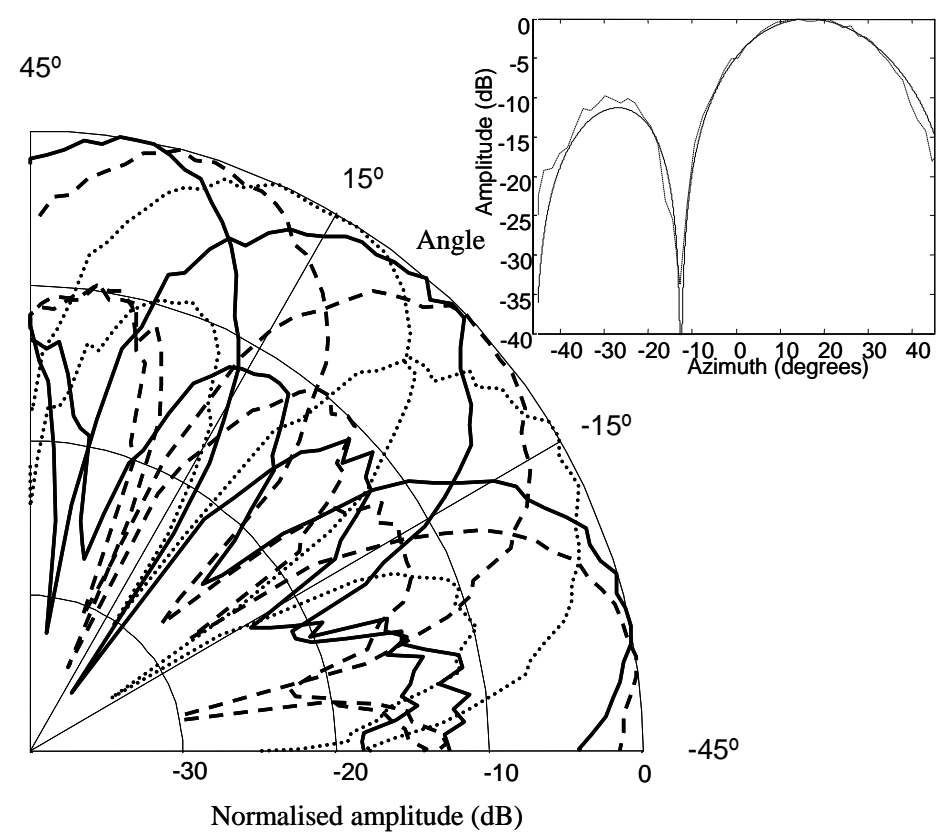

Figure 45. Measured radiation pattern at $40 \mathrm{GHz}$ for the 8 be am positions in transmission. Inset: (Dashed) Measured radiation pattern corresponding to the s witching state $0000(340 \mathrm{~m})$, (solid) theoretical radiation pattern. 
Figure 45 shows the measured radiation patterns corresponding to the delay and steering angles of Table 5. The experimental results agree quite well with theory as can be seen in the inset of Figure 45 where the measured radiation pattem corresponding to the switching matrix state $0000(340 \mathrm{~m})$ is shown and compared with theory. The measured $-3 \mathrm{~dB}$ beamwidth also agrees very well with theory.

The feasibility of the proposed optically beamformed antenna in transmission mode was confirmed by radio transmission experiments in the $40 \mathrm{GHz}$ band. A 32-QAM $155 \mathrm{Mb} / \mathrm{s} 1 \mathrm{GHz}$ signal was generated using a broadband modem board.

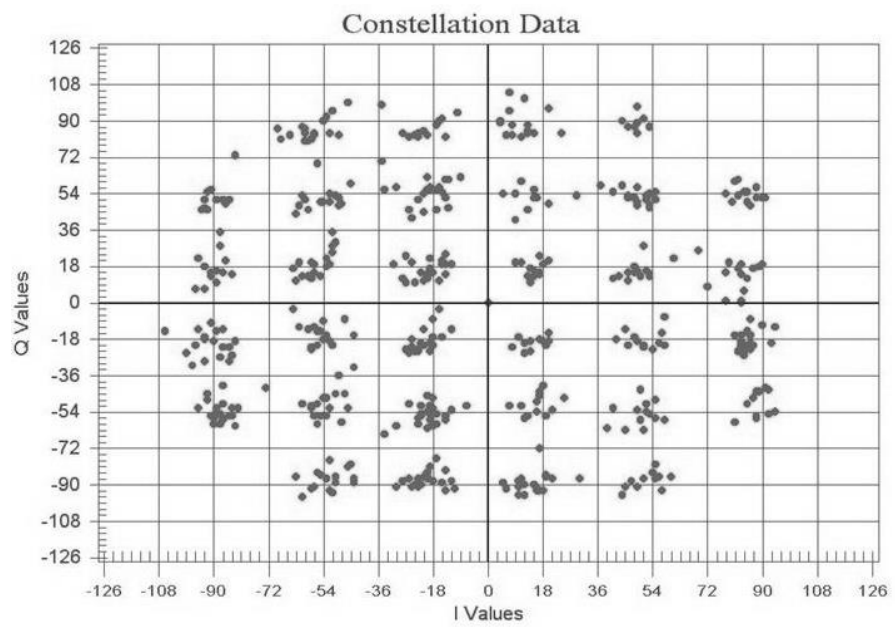

Figure 46. Constellation of the $155 \mathrm{Mb} / \mathrm{s} 32-\mathrm{QAM}$ after de tection (Tx mode).

Figure 46 shows the measured constellation diagram after detection. Error-free operation was achieved using 255/256 Reed-Solomon forward error correction codes.

The experimental setup SNR was limited due to the $1 \mathrm{~dB}$ interception point of the upconverter, which imposed a maximum $+7 \mathrm{dBm}$ output power level for distortion-free operation. The SNR can be improved by using a higher linearity upconverter; nevertheless, the optical beamformer is almost transparent, introducing a SNR degradation of $1.5 \mathrm{~dB}$.

\subsubsection{Reception Mode}

The experimental setup of the 3-bit beamformer in the reception mode is shown in Figure 47, which is similar to the setup in transmission where the input and output data spectrum can be seen in the inset. 


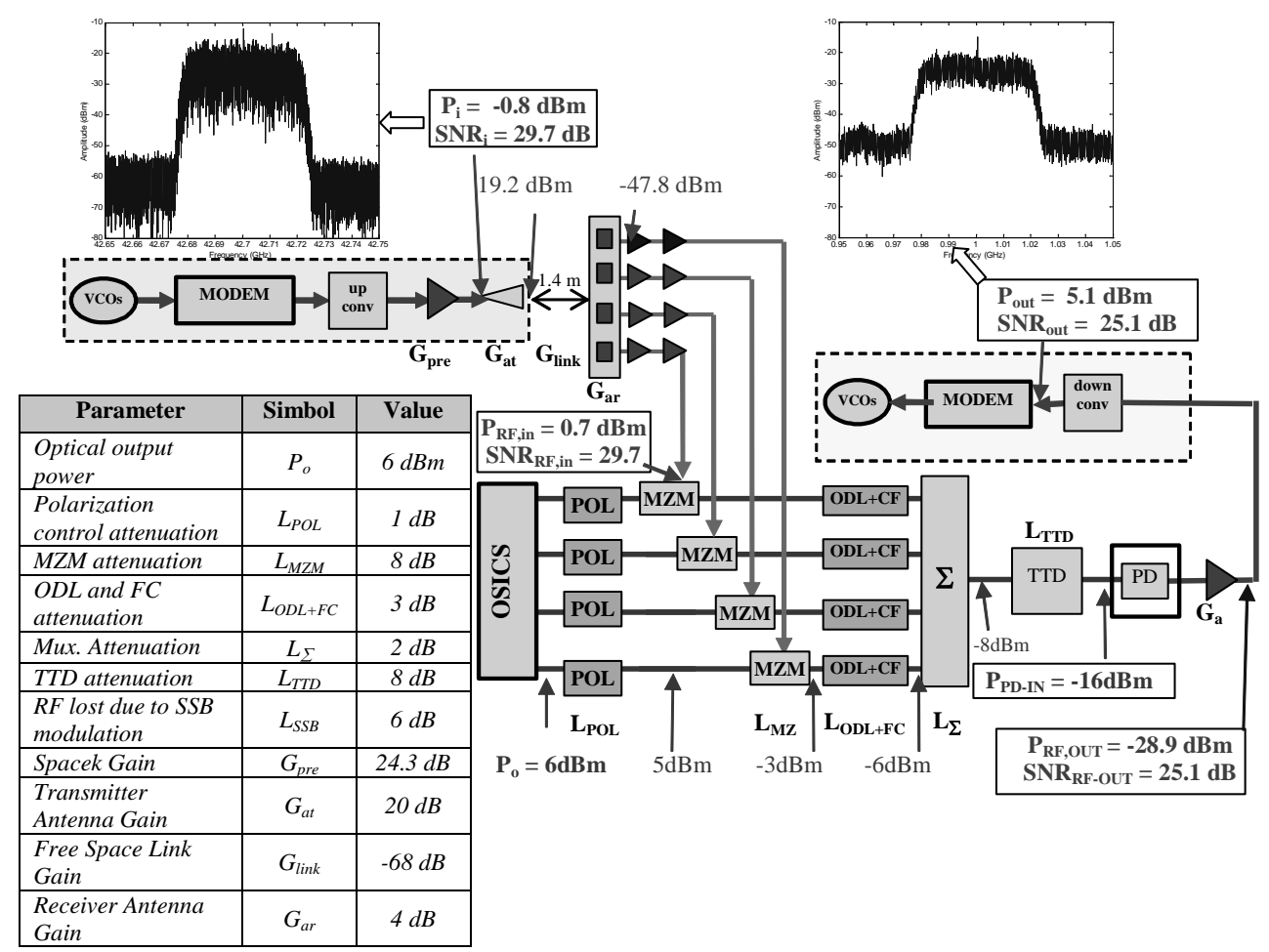

Figure 47. Experimental s etup for the reception mode.

Using a modem arbitrary board, a $155 \mathrm{Mb} / \mathrm{s}$ 32-QAM modulating signal at $1 \mathrm{GHz}$ was generated. Then, this signal is up-converted to $42.7 \mathrm{GHz}$ before being radiated. The received signal at the array antenna elements is translated to the optical domain using four MZM (four element antenna array). The lengths of the SSMF fibre coils are the same used in the transmission experiment $(170 \mathrm{~m}, 340 \mathrm{~m}$ and $680 \mathrm{~m}$ ). Finally, the four optical modulated carriers are photodetected using a single photodiode. The detected signal is down-converted to IF and demodulated.

The beam patterns for the eight beam steering angles have been measured, as shown in Figure 48, where theoretical predictions are not shown for the sake of clarity.

Several modulation formats (QPSK, 8PSK, 16QAM and 32QAM) have been evaluated to demonstrate the beamforming capability for high data rate transmissions. Error free operation was obtained for all modulation formats except for 32-QAM for which a BER of $10^{-6}$ was obtained using Reed-Solomon and convolutional codes $(4 / 5)$ when a payload rate of $155 \mathrm{Mb} / \mathrm{s}$ was used. Figure 49 
shows the measured constellation diagram after detection for $155 \mathrm{Mb} / \mathrm{s}$ 32QAM modulation.

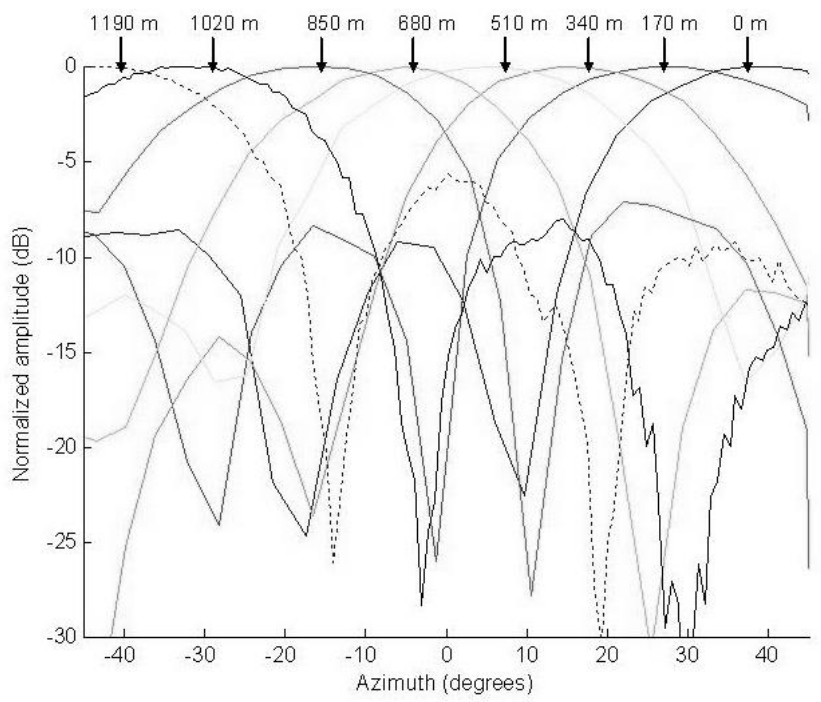

Figure 48. Measured antenna radiation pattern over the 90 degrees sector in receiving mode.

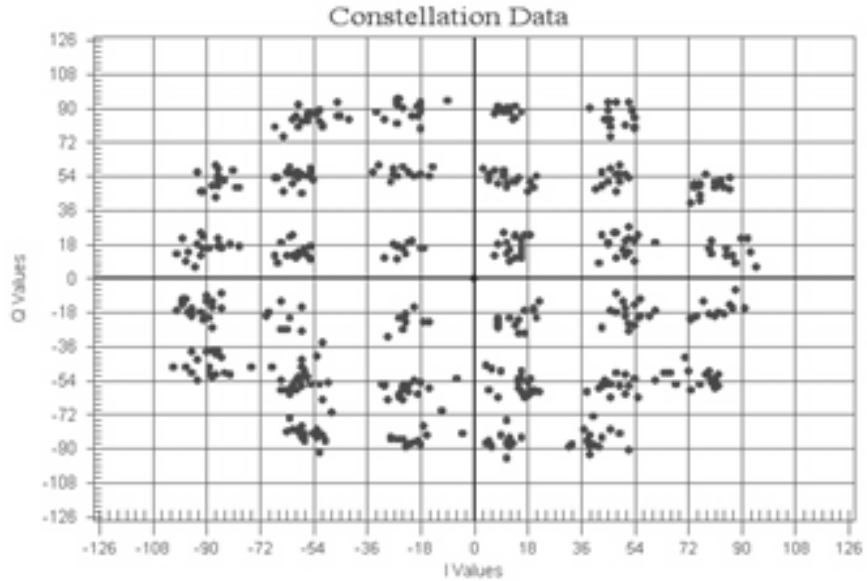

Figure 49. Constellation of the $155 \mathrm{Mb} / \mathrm{s} 32-\mathrm{QAM}$ after de tection ( $R x$ mode).

The measured SNR at output was $25 \mathrm{~dB}$ and was limited due to the $1 \mathrm{~dB}$ interception point of the upconverter, which imposed a low output power limit for distortion-free operation. From the measured output SNR it can be derived that the optical beamformer introduces a SNR reduction of around $4 \mathrm{~dB}$. Since the SNR is 
mainly limited by the electrical subsystem, it can be improved by using an upconverter with higher linearity.

\subsection{On the Capability of Multibeam Operation}

One of the main goals in optical beamforming networks is to reduce the complexity of the feeding network to reduce cost and bulkiness. One way to achieve this objective is through WDM which allow the combination of a multiwavelength laser and a single dispersive element to implement the whole network as described in the previous sections. A further step is the design of architectures with the capability to handle several beams simultaneously. If the beamformer has to provide several simultaneous beams, the time delay generation subsystem has to be replicated as many times as the number of beams. Finding ways to allow this capability while adding the minimum complexity will increase the competitiveness of optical solutions over their pure electronic counterparts.

In this section, an optical delay line based on tuneable notch filters is proposed as a way to allow multibeam beamformers with many of the advantages of switch-based architectures like integration potential and simplicity.

\subsubsection{Architecture Description}

The architecture is based on optical tuneable notch filters as shown in Figure 50, where a beamformer with two beams has been assumed for the sake of clarity. In the beamformer, one optical carrier is needed for each simultaneous beam. Each optical carrier is amplitude modulated with the data signal to be transmitted by each beam. This modulation can be done directly over the laser or externally using a Mach-Zehnder or an eletroabsorption modulator. Using a coupler, the signals are launched to a set of optical delay lines. There are as many delay lines as antenna elements. Each optical delay line is implemented by using $2 \times 2$ couplers and notch filters.

The number of filters at the output of each coupler is given by the number of antenna beams. Similarly, the number of couplers is given by the number of bits or time delays of the architecture (i.e. the number of discrete beam steering angles). The path length crossed by each optical carrier - corresponding with each beamcan be independently changed by controlling the centre frequency of the notch filters, and therefore, by creating dynamically routes of different length for each wavelength, depending on whether the signal at each output of each coupler is notch-filtered or not. This can be done independently for each beam (i.e. optical carrier of different wavelength) and, even, different carriers can share several sections without interference. Therefore, the beamformer can be used to steer several independent beams simultaneously. 


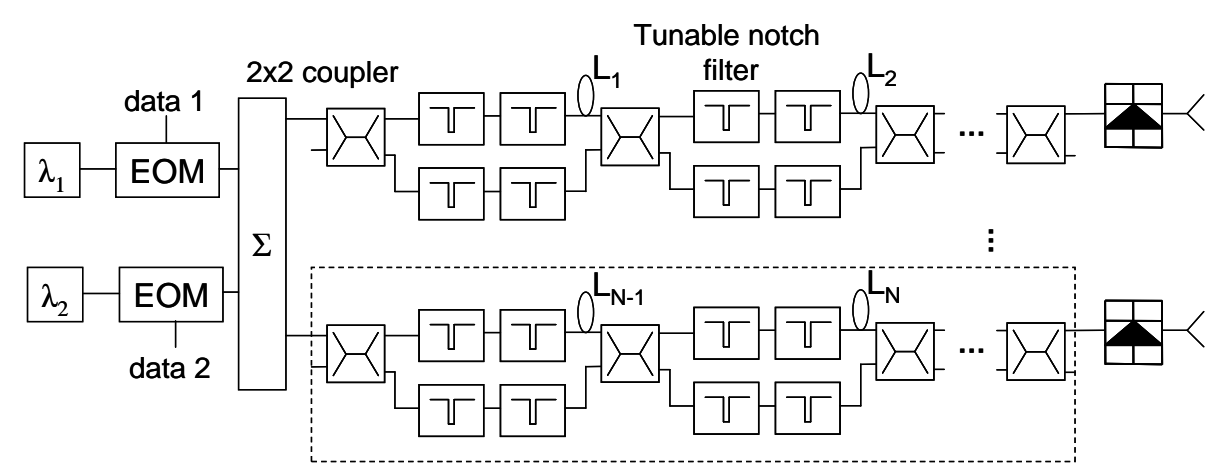

Switching matrix

Figure 50. B lock diagram of the multibeam beamforming ne twork bas ed on notch filters.

The multibeam capability of this architecture is done at the expense of higher insertion loss in comparison with architectures based on optical switches such as [GOU94], [TON96]. This is due to the use of couplers and notch filters instead of a set of optical switches.

This architecture can be used with non-dispersive media, as shown in Figure 50, or with dispersive media as in the architectures described in the previous sections. In the latter case, each beam uses a set of equally spaced optical carriers and the notch filters have to be periodic but, on the other hand, the number of switching matrices is reduced from as many as antenna elements to only one.

The use of notch filters instead of switches allows the implementation of multibeam architectures and has potential to be implemented in an integrated platform. Several tuneable integrated periodical notch filters on silicon, which can be tuned either electrically or optically, have been experimentally demonstrated (e.g. [ALM04], [XU05]). These devices show the potential to implement integrated multibeam optical beamformers at low cost thanks to be based on pervasive materials of the microelectronic industry.

\subsubsection{Experimental Results}

Proof-of-concept measurements have been carried out using one switching matrix. The experimental setup is shown in Figure 51. A tuneable laser was used as a CW laser, emulating a system of two beams (i.e. two optical carriers) since the vector network analyser cannot measure several time delays at the same time. Therefore, the time delays of each beam were measured non-simultaneously. The optical carrier was modulated using a MZM driven by a vector network analyser (HP8510C). The optical carrier was tuned to two different wavelengths: $1547.6 \mathrm{~nm}$ (beam \#1) and $1548.4 \mathrm{~nm}$ (beam \#2). Then, a switching matrix based on three 
couplers and four filters was implemented as shown in Figure 50. A combination of different filters (including tuneable gratings and Fabry-Perot filters) were used. The non-dispersive media was standard single mode fibre coils of approximate lengths $\mathrm{L}_{1}=1 \mathrm{~km}$ and $\mathrm{L}_{2}=5 \mathrm{~km}$, respectively. Therefore, there are four total lengths $0, \mathrm{~L}_{1}, \mathrm{~L}_{2}$ and $\mathrm{L}_{1}+\mathrm{L}_{2}$. Finally, the signal was photodetected and time delays measured using the network analyser.

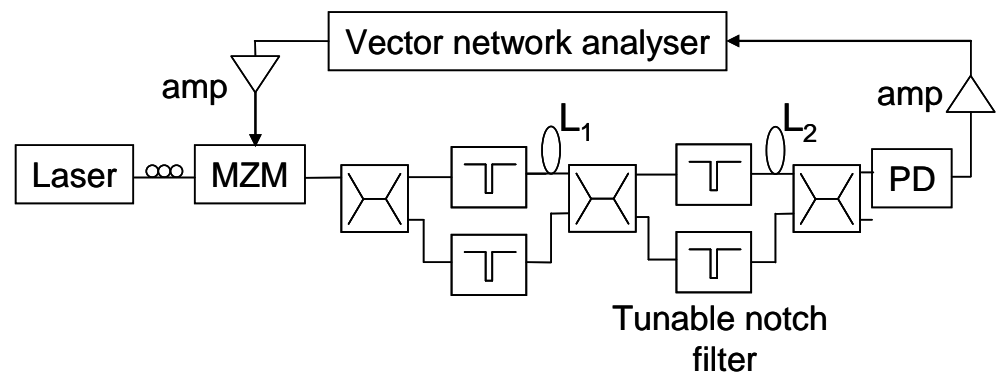

Figure 51. Experimental setup used to characterize the multibeam optical delay line bas ed on notch filters.

The time delay measurements were done from the relative phase slope between 2 and $6 \mathrm{GHz}$. Several different delays were measured to show that a notch-based optical delay line with two optical carriers has the capability to generate two sets of independent time delays.

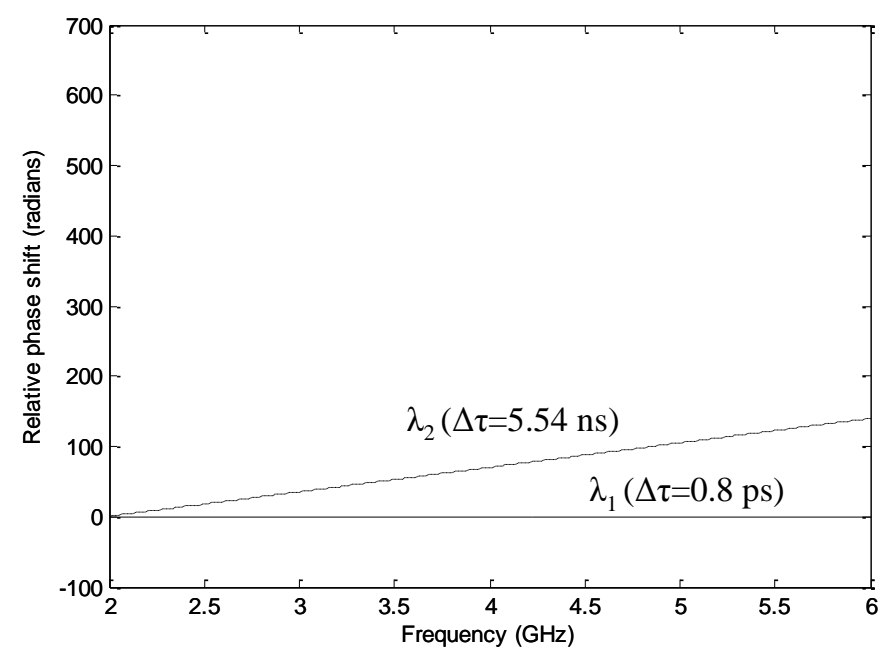

Figure 52. Time delay measure ments for beam \#1 (length: $0 \mathrm{~m}$ ) and be am \#2 (length: $\mathrm{L}_{1}$ ). 


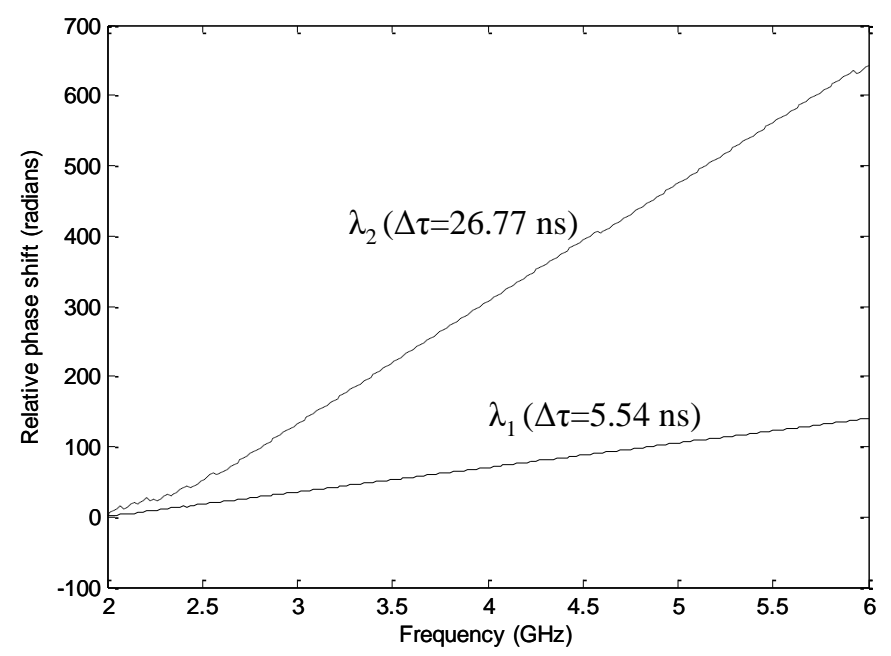

Figure 53. Time delay measure ments for beam \#1 (length: $L_{1}$ ) and beam \#2 (length: $\mathrm{L}_{2}$ ).

Figure 52 and Figure 53 show an example of two sets of time delays measurements corresponding to beams \#1 and \#2 crossing through path lengths 0 (beam \#1) and $\mathrm{L}_{1}$ (beam \#2) in Figure 52 and lengths $\mathrm{L}_{1}$ (beam \#1) and $\mathrm{L}_{2}$ (beam \#2) in Figure 53. As can be seen, the time delay for each beam (i.e. each optical carrier) can be chosen independently of other beams (unlike previous architectures based on optical switches). Therefore, the proposed optical delay line can be used for multibeam optical beamformers.

\subsection{Conclusion}

In this chapter a photonic beamformed beam-switched phased array antenna based on optical switches and dispersive media has been proposed and experimentally demonstrated in the $40 \mathrm{GHz}$ band both in transmission and reception modes. Error-free $\left(10^{-9}\right)$ transmission of $32-\mathrm{QAM} 155 \mathrm{Mb} / \mathrm{s}$ data at $42.7 \mathrm{GHz}$ has been achieved using the proposed optically beamforming network in transmission and an error rate of $10^{-6}$ was obtained in the receiving mode. In both cases, the optical beamformer is almost transparent introducing a small degradation over the SNR.

On the other hand, a modification of the previous architecture for multibeam optical beamforming based on tuneable notch filters with independent beam control has been proposed. The architecture offers the capability to implement compact multibeam architectures and has the potential to be highly integrated. Experimental results to proof the concept have been presented. 


\section{CHAPTER 4}

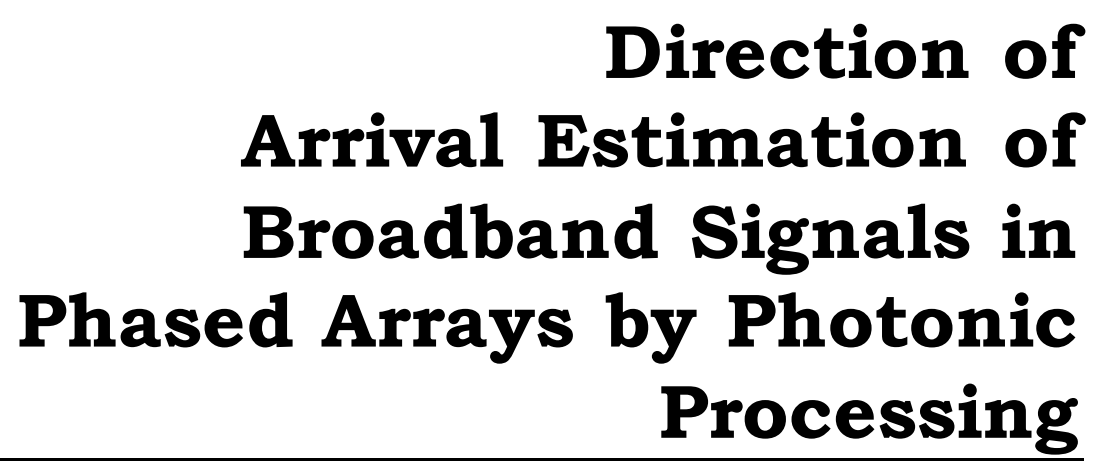

In addition to the basic operations of beamforming and beam steering, the optical implementation of additional functionalities, such as nulling [MAT99], [MEN09] and multibeam capability, as described in the previous section, has been also proposed. The implementation of these functions using photonic components is needed to maintain the advantages of the optical beamformer (i.e. the capability to transmit/receive broadband signals without beamsquint using true time-delay, remote feeding, etc).

Another interesting functionality which can be implemented using optics is the direction of arrival (DOA) estimation. DOA information can be used to minimize the signal power of the interference while maximizing the power of the desired signal, improving the performance of the system. Therefore, DOA is a very interesting capability for broadband mobile systems and electronic warfare scenarios. DOA estimation is usually performed with digital signal processing techniques [GOD97]. However, digital signal processing of broadband signals at high microwave frequencies is difficult due to the bandwidth limitations of present 
digital signal processors. Therefore, optical implementations of DOA have been proposed [GAL97], [BIE98], [TON06]. However these proposals are rather complex or partially based on IF processing which limit their capability to process broadband signals ${ }^{7}$.

\subsection{Principle of Operation}

In phase array antennas in reception mode, the incoming signal at each antenna element arrives with a different time delay depending on the angle between the signal source and the antenna array due to the spatial separation between antenna elements (Figure 54).

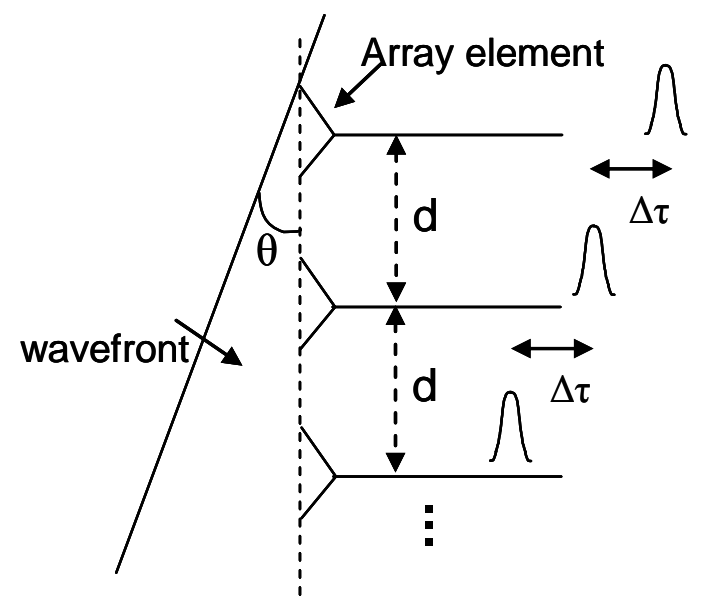

Figure 54. Incidence of a wave front on a line ar equally space d ante nna array.

From the relative time delay between the signals of each antenna element, it is possible to derive the angle of incidence and, therefore, the direction of the source. The angle of arrival is given from:

$$
\theta=\sin ^{-1}\left(\frac{c \cdot \Delta \tau}{d}\right)
$$

where $\Delta \tau$ is the time delay between antenna elements, $d$ is the spacing between antenna elements and $c$ is the speed of light in vacuum.

Therefore, the measurement of $\Delta \tau$ allows the estimation of the angle of arrival of the incoming signal. We propose the measurement of $\Delta \tau$ by means of a photonic

${ }^{7}$ The work described in this chapter has been published in the publication D 
transversal microwave filter. Photonic transversal microwave filters [ZHA98], [VID03b] are optical implementations of finite impulse response (FIR) filters based on optically delaying samples of electrical signals modulated over optical carriers. The filter response is periodic and the free-spectralrange (FSR) (i.e. the frequency period) is given by:

$$
F S R=\frac{1}{\Delta \tau}
$$

As can be seen in equation (4.2), measuring the FSR, the time delay between the filter samples can be obtained. This fact can be used to determine the DOA of broadband signals, especially in optical beamforming networks where just a few additional components will be needed by using a photonic microwave notch filter since the electro-optic conversion is already done for the beamforming task. The frequency of the notches over the broadband signal is measured to determine $\Delta \tau$.

\subsection{Architecture Description}

Figure 55 represents a general model of an optical beamformer in reception mode. As can be seen, the microwave signals modulate the optical carriers by means of external modulators. Then, the optical signals enter to the TTD unit, which can be implemented in different ways. It introduces a time delay between the signals of each antenna element in such a way that, at its output, the signals are in phase. Finally, the signals are photodetected.

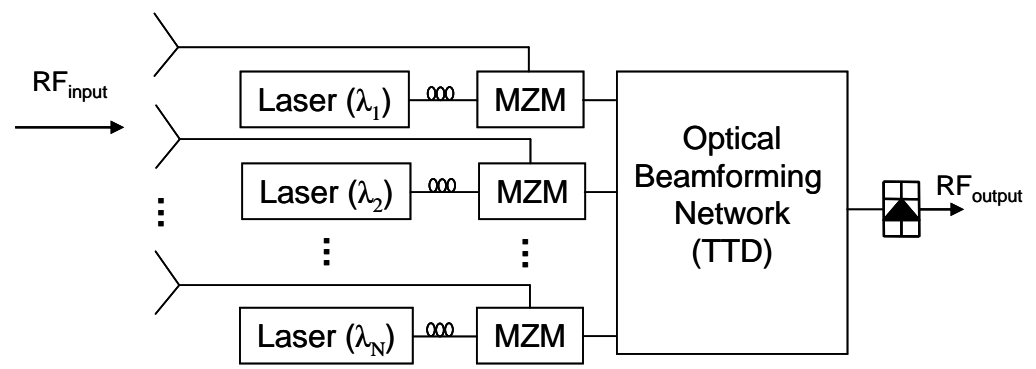

Figure 55. Ge neral model of an optical be amforme r in re ce ption mode. MZM: Mach-Zehnder Modulator, TTD: True time-delay.

To determine the DOA, the previous scheme is slightly modified to combine the output of two antenna elements. After the external modulators, the two signals are divided between the optical beamformer and the DOA estimation subsystem using two optical couplers. 


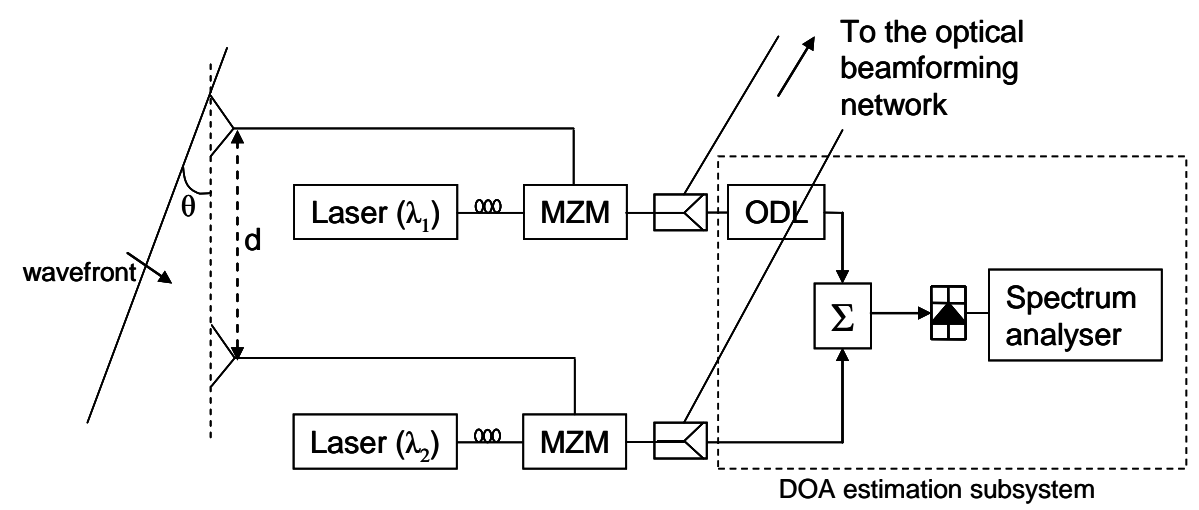

Figure 56. DOA detection using a photonic microwave filter in an optical be amformer. ODL: Optical delay line.

After photodetection, the combination of two samples of the same microwave signal with a time delay between them creates a notch filter. The frequencies of the notches are given by [ZHA98],

$$
f_{\text {notch }}=(k+0.5) \frac{1}{\tau}
$$

where $k=0,1,2,3 \ldots$ and $\tau$ is the time delay between the two branches due to its fibre length difference. This time delay can be changed, for instance by using an optical delay line (ODL) or a fibre patch cord with a proper length, to adjust the frequency and the spacing between notches (i.e. the FSR) and to match the notches with the bandwidth of the microwave signal. A change in the time delay $(\Delta \tau)$ given by angles of incidence of the incoming signal different from broadside will change the frequency of the notches [ZHA98] as:

$$
\Delta f_{\text {notch }}=\frac{(k+0.5)}{\tau^{2}} \Delta \tau
$$

Using, for instance, a network analyser, the frequency of the notches over the microwave signal can be measured and from its frequency, the time delay and the angle of arrival can be estimated. The frequency of the notches could be detected also using high-Q tuneable optical filters (such as in-fibre Fabry-Perot filters) or photonic microwave filters.

This technique can be used only with broadband signals since the DOA measurement is done over the signal and a certain bandwidth is needed to allow the notch excursion (as shown in Figure 57, where it can be seen how the broadband 
signal is filtered by a transversal filter generated combining the signal from two antennas and how the filter response changes when the emitter moves). However, this is not a limitation since the main aim of optical beamformers is the control of phased array antennas when using broadband signals. On the other side, the time delay between branches $(\tau)$ has to be adjusted to allow the matching of the frequency of the notches as well as their excursion with the bandwidth of the microwave signal. Once the nominal time delay between branches $(\tau)$ is adjusted, it remains fixed.

Prior to the estimation of the DOA it is necessary to know the time delay between branches $(\tau)$ to allow the determination of the angle of incidence from the changes in the notch frequencies as given by eq. (4.4).

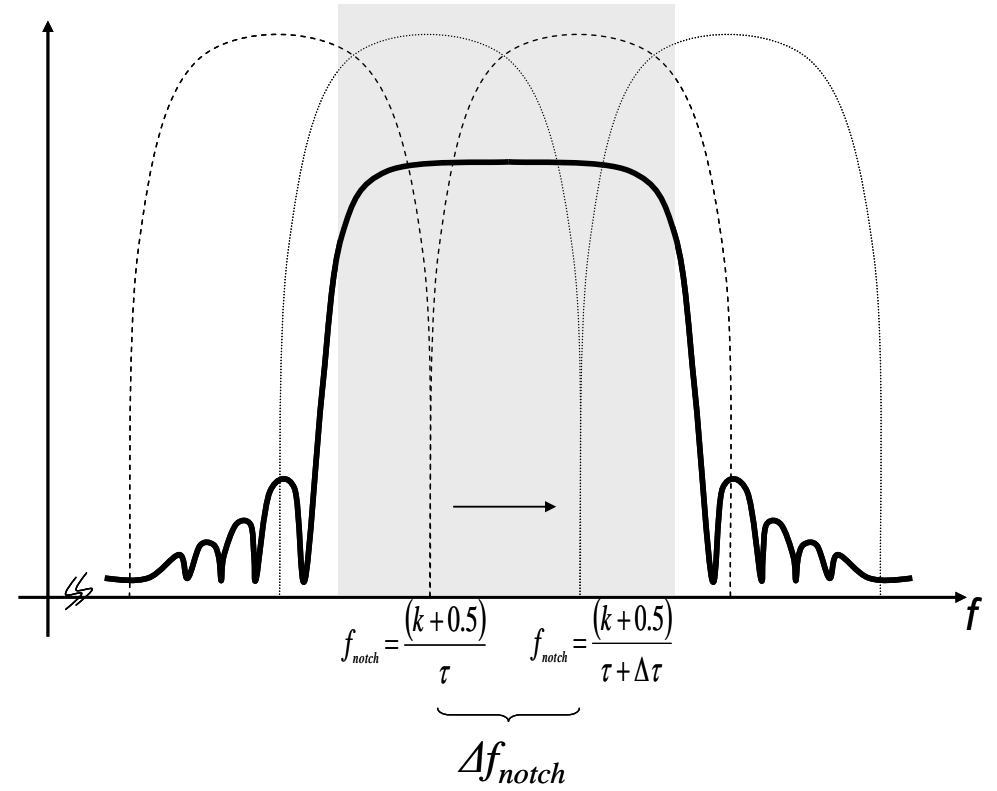

Figure 57. Concept of DOA measurement using a photonic trans versal microwave filter. The solid line represents the spectrum of a broadband signal, the dotted line the trans versal filter res ponse generated when the signals from two antennas are combined and the light dotted line the filter response when the emitter has changed its position.

The discrimination between positive and negative angles is straightforward using this technique. If a positive angle increases the total time delay between the two samples $(\tau+\Delta \tau)$, negative ones will reduce the time delay $(\tau-\Delta \tau)$, and therefore, the spacing between notches will be increased or decreased. 
In addition, this technique of DOA estimation can be used in scenarios with several simultaneous emitters. If the time delay $\tau$ is adjusted in such a way that there is only one notch over the signal bandwidth, the number of notches indicates the number of emitters and from the frequency of each notch the DOA of each emitter can be derived.

\subsection{Experimental Results}

In order to validate the technique described in the previous sections the experimental setup of Figure 58 has been used.

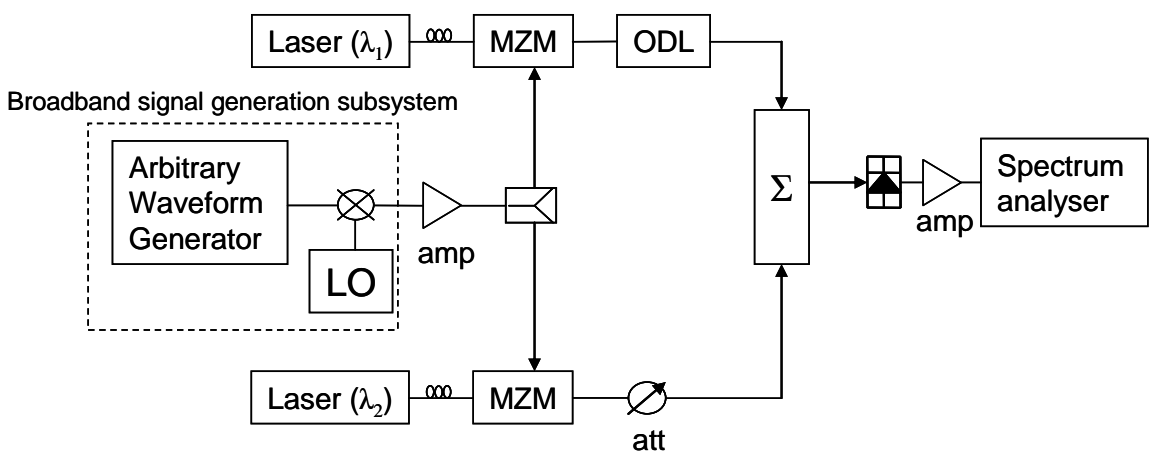

Figure 58. Experimental s etup used to show the feasibility of the proposed te chnique (LO: Local oscillator, att: attenuator, amp: amplifier).

A $1.25 \mathrm{~Gb} / \mathrm{s} \mathrm{NRZ}$ signal has been generated by using an arbitrary waveform generator. This signal has been up-converted with a mixer to $10 \mathrm{GHz}$. The spectrum of this broadband signal $\left(\Delta \mathrm{B}_{-3 \mathrm{~dB}}\right.$ of $1 \mathrm{GHz}$ corresponding to a null to null bandwidth is $2.5 \mathrm{GHz}$ ) is shown in Figure 59. The signal is used to amplitude modulate two optical carriers by means of two Mach-Zehnder modulators. An optical delay line is used to adjust the time delay between branches. In order to simplify the setup, the effect of changes of the angle of arrival of the incoming signals has been emulated with the optical delay line which allows the change of the time delay between both branches. An attenuator is used to equalize the amplitude of the signal of each branch and thus improve the nulls depth. Then, the signals are combined and photodetected. 


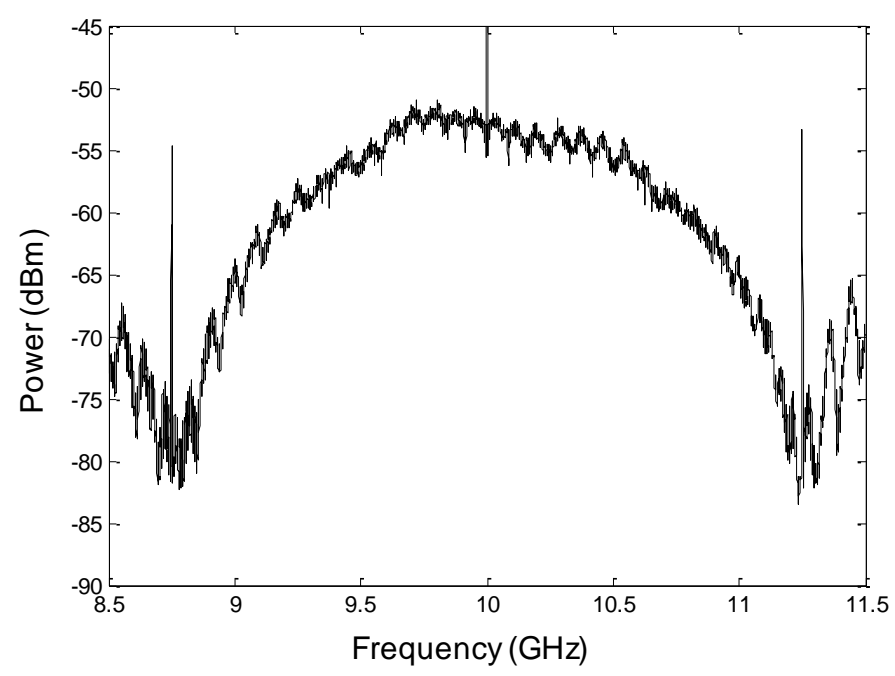

Figure 59. Spectrum of a $1 \mathrm{GHz}-3 \mathrm{~dB}$ bandwidth signal centred at $10 \mathrm{GHz}$.

The frequency of the notches and their variation when the time delay is changed has been measured using a spectrum analyser. Two different sets of measurements have been carried out with two different time delays corresponding to different lengths between the branches $(\tau)$. To do it, an additional short fibre patch cord has been added. It results in a single notch in the signal bandwidth or three notches, i.e. a smaller $\tau$ (a larger FSR) or a larger $\tau$ (a smaller FSR). Figure 60 and Figure 61 show the spectra of the broadband signal after being filtered as well as the original unfiltered signal. 


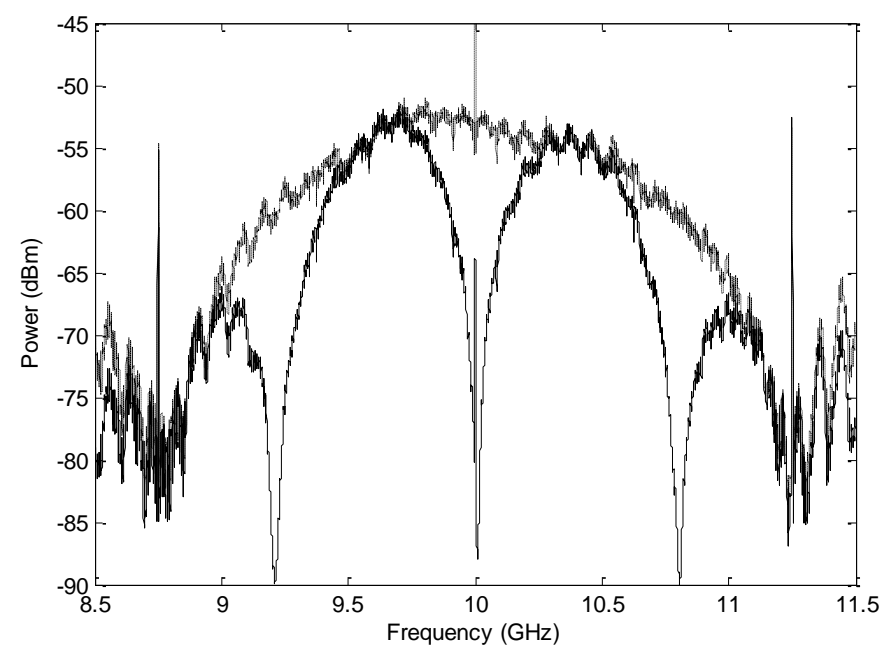

Figure 60. Broadband signal (1 GHz -3 dB bandwidth) with three notches (solid) and the original unfiltered signal (dotted).

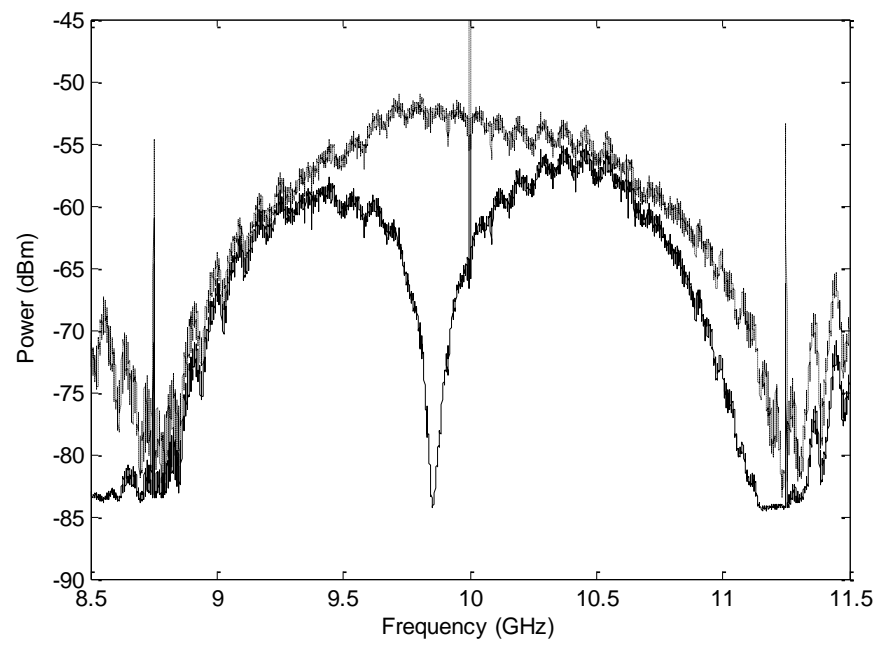

Figure 61. Broadband signal ( $1 \mathrm{GHz}-3 \mathrm{~dB}$ _bandwidth) with a single notch and the original unfilte red signal (dotted).

To measure the time delay due to the length difference between the two branches $(\tau)$, the frequency of the notches has been measured by changing the time delay $(\Delta \tau)$ introduced by the ODL. This calibration allows the measurement of the time delay $\tau$. Figure 62 and Figure 63 show the relation between the offset of the notches and 
the time delay introduced by the ODL or the equivalent angle of arrival (derived from (4.1) assuming an inter-element spacing of $0.5 \lambda$ ) for three and one notches, respectively, as well as a least square interpolation used to determine the coefficient of (4.4). As can be seen, the results show a good linearity.

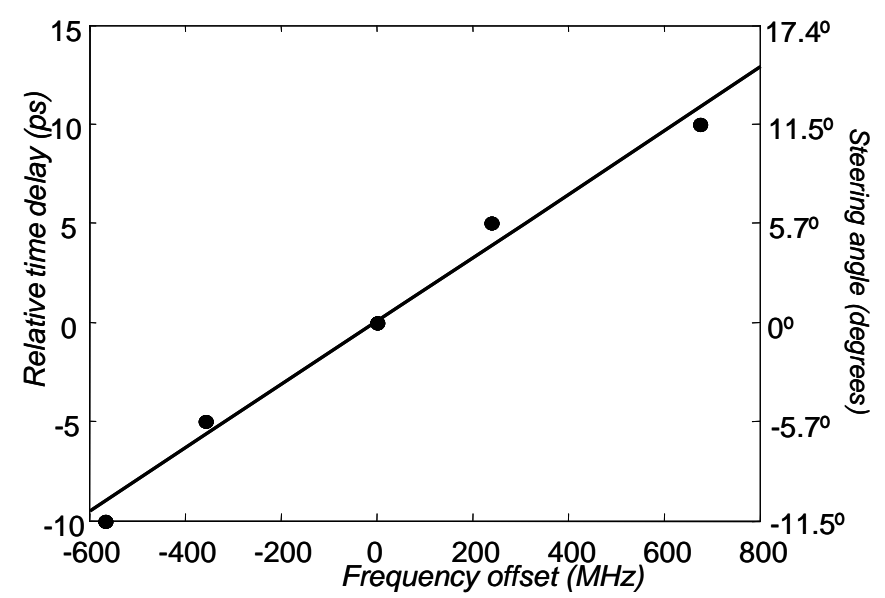

Figure 62. Measurement of the relation between the offs et of the notch and the re lative time delay between antenna elements/angle of arrival for the setup

showing three notches.

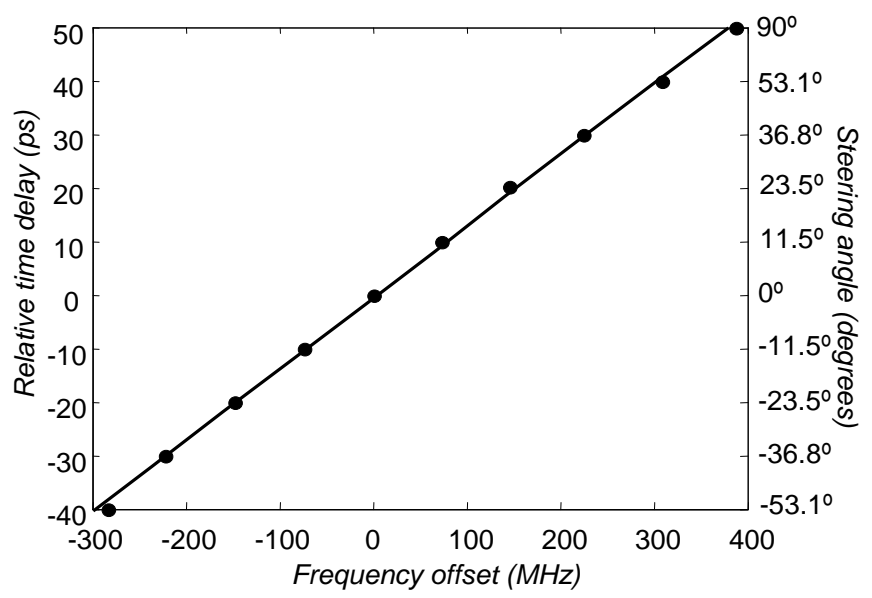

Figure 63. Measurement of the relation between the offs et of the notch and the relative time delay between antenna elements/angle of arrival for the setup showing a single notch. 
From Figure 62 and Figure 63, it can be seen that with a smaller $\tau$, the effect of changes in the angle of arrival is more noticeable (the change of the offset of the notches is more marked). It means that the sensibility of the system is greater but the range of angles of arrival that can be discriminate is smaller. The range of angles of arrival is given by the bandwidth of microwave signal or by the filter FSR. The notches can be measured only in a bandwidth equal to the filter FSR to avoid ambiguity in the frequency measurement.

To show the feasibility of the technique after the calibration, in the setup configuration that corresponds with three notches in the microwave signal, a time delay value has been introduced by means of the ODL, $\Delta \tau=5 \mathrm{ps}$. The frequency of one of the notches was measured with the spectrum analyzer to be $9.7767 \mathrm{GHz}$, which means an offset $\Delta f_{\text {notch }}=0.0312 \mathrm{MHz}$ from $9.7455 \mathrm{GHz}$ which is the central frequency of this notch. From Figure 62, the slope of the least square approximation can be measured, $\tau^{2} /(k+0.5)=133.37 p s^{2}$. From this value, the measured time delay is $4.1 \mathrm{ps}$ whereas the expected time delay should be around $5 \mathrm{ps}$. Additional measurements have been carried out showing similar performance. Table 10 shows three measurements and the expected time delays, showing an excellent agreement between them.

TABLE 10: TIME DELAY MEAS UREMENTS AND THE EXPECTED VALUE.

\begin{tabular}{|c|c|c|}
\hline Measured notch frequency & Measured time delay & ODL time delay \\
\hline $9.7767 \mathrm{GHz}$ & $4.1 \mathrm{ps}$ & $5 \mathrm{ps}$ \\
\hline $9.8545 \mathrm{GHz}$ & $14.4 \mathrm{ps}$ & $15 \mathrm{ps}$ \\
\hline $10.0085 \mathrm{GHz}$ & $35.07 \mathrm{ps}$ & $35 \mathrm{ps}$ \\
\hline
\end{tabular}

These measurements validate the feasibility of the DOA estimation technique, showing that the time delay and from it, the angle of arrival can be measured easily in optical beamformers. In the case of lower signal-to-noise ratio (SNR), data processing would be needed to estimate the notch frequency. A simple processing algorithm was implemented to assess the effect of noise over the accuracy of the DOA estimation although it actually depends on several parameters such as the frequency and the spacing between antenna elements. For a SNR of 10 and $20 \mathrm{~dB}$, and assuming a central frequency of $10 \mathrm{GHz}$ and an inter-element spacing of $0.5 \lambda$, the accuracy in the DOA estimation was around 5 degrees and 1 degree, respectively. 


\subsection{Conclusion}

A simple technique to measure the direction of arrival of broadband signals in optical beamformers for phased array antennas has been proposed. The technique is based on a two-tap photonic transversal microwave filter. In fact, it is a new application for this kind of optical processing structures. It has been shown that DOA estimation can be done with a slight modification of an optical beamformer, adding this interesting functionality with just a few additional cost-effective photonic components and without limiting the beamformer capability of process broadband signals. In addition, the DOA of several simultaneous sources could be easily determined without adding additional complexity to the system. The technique has been experimentally demonstrated and the experimental results, using signals of good SNR, show a good agreement with the expected results. Finally, although in this thesis the main focus has been the DOA estimation, the proposed technique can be used in general to do time delay measurements in different applications ${ }^{8}$.

${ }^{8}$ This work has been published in the publication D. 


\section{CHAPTER 5}

\section{Heterodyne \\ OBFN based on Integrated Butler Matrices}

In this chapter, a heterodyne integrated optical Butler Matrix is presented and demonstrated ${ }^{9}$. As described in chapter 2, the Butler Matrix is a passive microwave structure that performs a discrete Fourier transformation over the input signal, which is known from the sixties. Thanks to its simplicity and its multibeam capacity it has been used as passive beamforming network in beam-switched array antenna systems. This structure, although is interesting for beamforming applications, presents some disadvantages when implemented on-board of satellites such as its weight and susceptibility to electromagnetic interferences. These problems become more important when the number of antenna elements increases as in systems with high beamforming/beam-switching capabilities with several orthogonal beams like the ones considered for the next generation of broadband communication.

In this case, integrated photonic technology can play a crucial role as an alternative technology for implementing such beamforming structures for satellite applications thanks to the well-known advantages of this technology such as electro-magnetic interference immunity, low consumption, low volume and weight, huge electrical bandwidth, low-attenuation, remote delivery capability (thanks to the optical fibre) and the robustness and precision offered by integrated technologies,

${ }^{9}$ This research has been done in the frame of an ESA contract in collaboration with Thales Alenia Space (France), in charge of the antenna concept top level definition and the description of the application scenario, and the Nanophotonics Technology Center from the Universitat Politècnica de València in charge of the manufacturing of the SOI designs. 
especially in the case of Silicon-on-Insulator (SOI), thanks to the compactness and its compatibility with the standard CMOS technology.

In this chapter, the development of a nanophotonic structure that implements an optical Butler matrix of $8 \times 8$ ports is described, which is envisaged to be the core of a ultra-compact Optical Beamforming Network capable to cope the requirement of the new generation of communication satellites, which exploits the advantages of the abovementioned photonic technology. The Butler matrix chip has been designed, fabricated and tested in SOI technology.

\subsection{Application Scenario}

The study of optical Butler Matrices is going to target the control of an array of antennas for broadband communications in satellites. Broadband systems should offer high data rate connections to very large numbers of low cost terminals. To be economically viable, the cost per bit shall be as low as possible. Only satellites with very large capacity in terms of bandwidth or number of circuits would achieve the required low cost for commercial deployment. Therefore, there is a need for new technologies to allow reaching this target.

Thus, the work is going to be aimed at a real scenario consisting in a typical communication satellite based on a geostationary platform covering Europe and the Maghreb with 44 spots (beams) with an angular separation of 0.8 degrees and distributed in a 6 rows by 8 spots grid. With this scheme, different options are possible, either a classical frequency re-use scheme (FDD) or a beam-hopping/switching (TDD) architecture, both in a Direct Radiating Array configuration in downstream. The antenna array for obtaining the required radiation characteristics consists on a 256 elements array in a circular configuration, as shown in Figure 64.

The general design of the beamforming network for feeding an antenna array of 256 elements is depicted in Figure 65. It consists of a 2-dimensional network with an input stage of six 8x16 matrices (each row of the coverage distribution corresponds with each input matrix), with 48 inputs (corresponding with 48 orthogonal beams, although some of them are unused) and $6 \times 16=96$ outputs. These outputs feed a second set of 16 truncated $8 \times 16$ matrixes (only 6 inputs are used in each matrix) resulting in $16 \times 16=256$ outputs, which directly feed the antenna elements. 


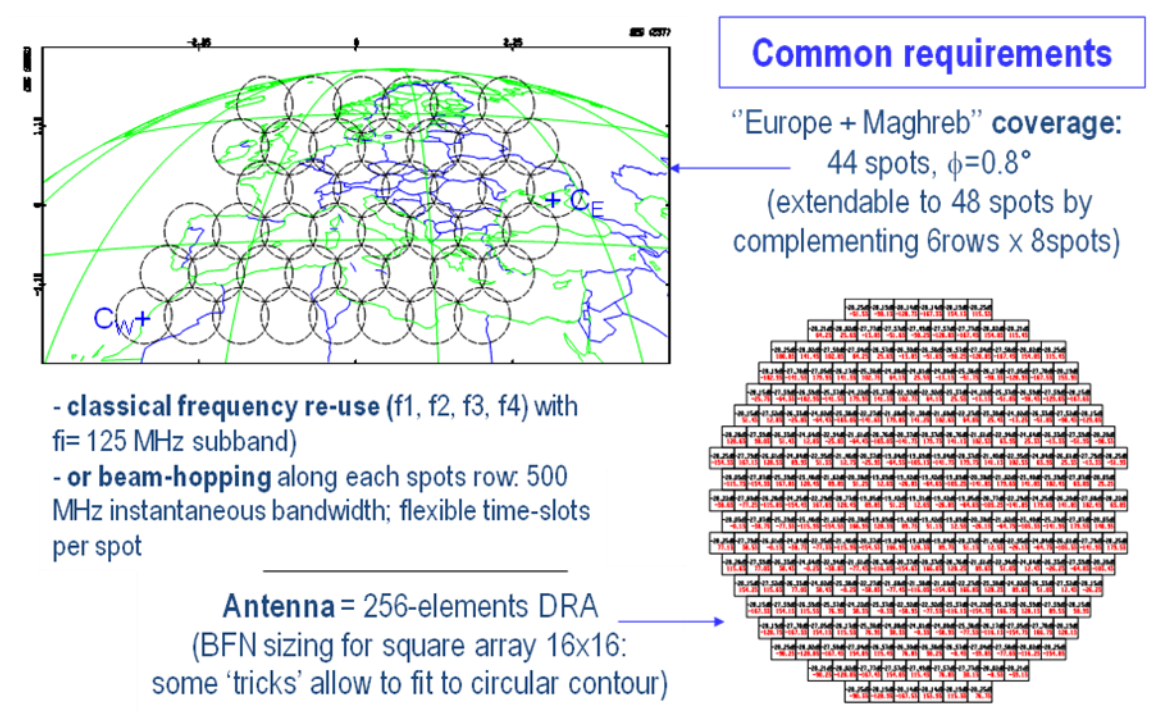

Figure 64. Application scenario.

This structure implements both a heterodyne generation (differential phase shifting in each spectral component by routing each one through different physical path) and a $2 \mathrm{~N}$ extension capability [MAD02]. It consists of two 8x8 optical Butler matrices working in parallel (16 outputs). These are connected to the 8 inputs through a set of couplers and phase shifters. At the input, the sideband of the optical field is extracted (Sideband extraction block) and routed to the output (normal configuration) and to the input port \#1 ( $2 \mathrm{~N}$ configuration) depending on the selected input.

In particular, the system carries out the following tasks over the input signal which is an optical signal with single sideband or double sideband modulation, which carries the RF signal to be transmitted:

- The matrix split the input signal at each input port into 16 outputs.

- The phase difference between the optical carrier and the sideband(s) at each output port depends on the input port.

- When photodetected, this phase difference is translated to the obtained RF signal. It generates the required phase profile at the antenna elements for obtaining a maximum of radiation in the desired space direction.

- Further phase shifting can be obtained if the output optical signals are routed through more butler matrices (the phase shifts are summed). 


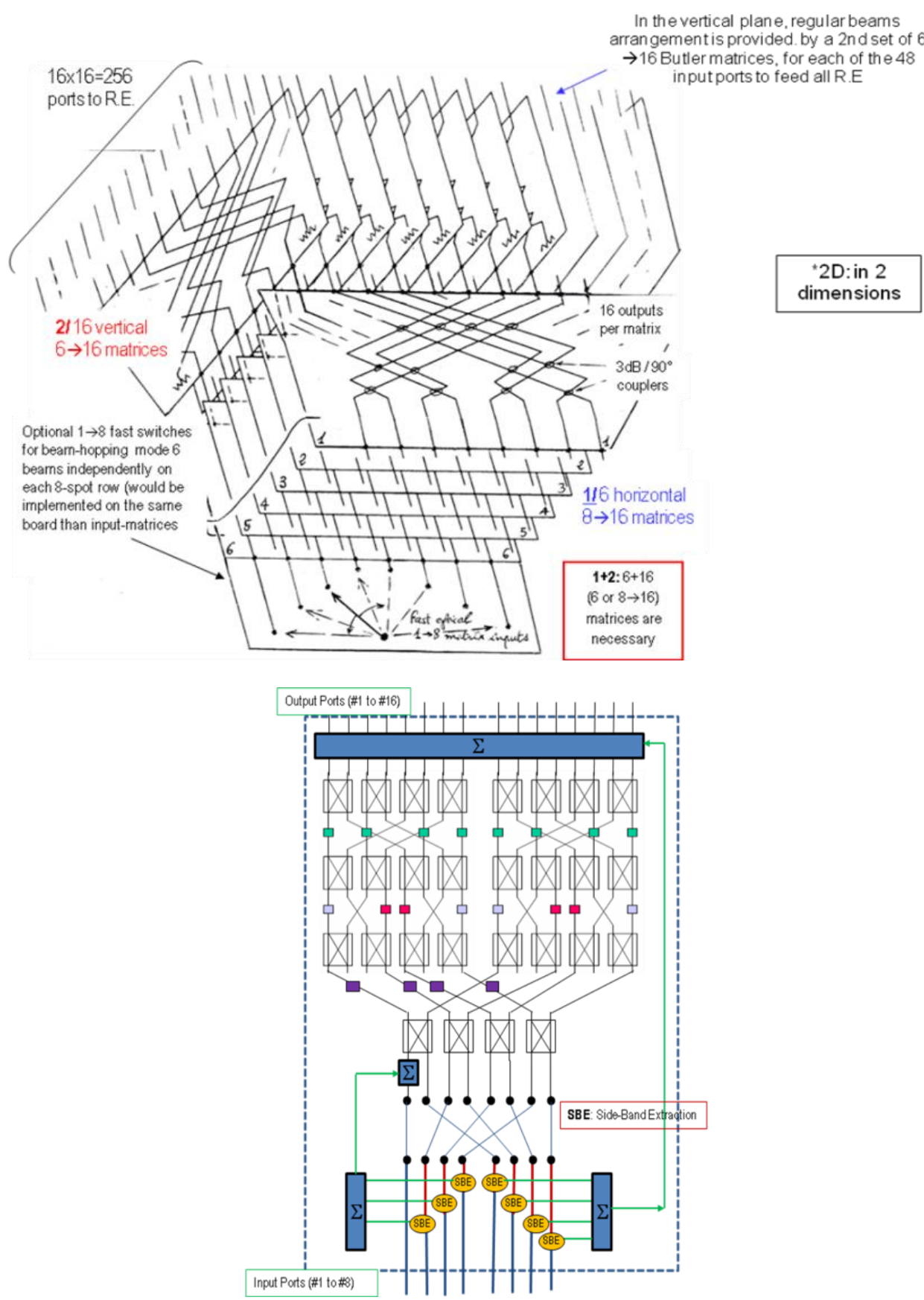

Figure 65. B eamforming network for the feeding of 256 elements by using $8 \times 16$ extended Optical Butler Matrices (up). 8x16 matrix employed in the beamforming network consisting in an extension of the $8 \times 8$ Optical B utler Matrix employing a scheme for the extension of the number of beams (down). 


\subsection{Optical Butler Matrices for Electrical Beamforming}

As described in section 1.6.4, one of the traditional electrical methods of feeding an array antenna with a uniform distribution and constant phase difference between elements is by means of a Butler matrix. This matrix, first described by Butler [BUT61], consists of $N$ input ports and an equal number of output ports which feed each radiating element. A signal introduced and one input produces equal amplitude excitations at all outputs but with a defined phase difference between them, producing a maximum of radiation at certain angle in space (or beam steering). A signal introduced at another input results in radiation at another angle. A more general scheme for different angular beam positions is shown in chapter 2. The phase difference between radiating elements for a Butler Matrix with $N$ elements and for the $p^{\text {th }}$ beam location is given by [MOO64]:

$$
\psi_{n}=\frac{2 \pi d}{\lambda} \cos (\alpha)
$$

where $d$ is the separation between adjacent antenna elements, $\lambda$ is the wavelength of the signal, $\alpha$ is the angular position of the $p^{\text {th }}$ beam respect to the endfire direction and $\psi_{n}$ is positive or negative depending upon whether the beam is to right or left of broadside respectively.

The same design can be used not only in electrical but also in optical frequencies since the same building blocks employed in the electrical implementation can be fabricated for its optical counterpart. Effectively, a typical $3 \mathrm{~dB}$ optical coupler performs a 90 degrees' phase shifting between its outputs, resulting in an optical 90 degrees hybrid. Moreover, phase shifting can be achieved by controlling the length of an optical waveguide. Although there have been in the literature implementations of optical Butler matrices by using discrete components, the implementation of the Butler matrix in integrated optics technology is highly desired due to the high precision of the waveguide lengths required to implement precisely the necessary optical phase shifting. In this sense, the SOI technology is a promising solution since this technology is compatible with the traditional processes of the microelectronic industry. Moreover, thanks to the high index contrast of the SOI waveguide, resulting in devices very small compared with other integrated optics technologies and achieving a more precise control of the circuit size. Typically, microwave Butler matrices consisting of microwave fixed phase shifters and directional couplers have poor performance because of the high crosstalk and electromagnetic interference (EMI) [CHA90]. Integrated optical Butler matrices, in addition to being immune to EMI and having negligible crosstalk are small in size, lightweight and can be manufactured on a single substrate. 


\subsubsection{Principle of Operation: Heterodyne Phase Shifting}

The optical Butler matrix replicates the optical input signal at all the outputs, inducing a different phase shift to each output. The way to translate such phase shifting to the electrical signals is by using optical heterodyne generation. Let it be two monochromatic coherent optical waves with a frequency difference of $f_{R F}$ travelling together in the same optical fibre. By coherent it is understood that the optical phases of both signals maintain a constant phase shift. On the other hand, they are incoherent when this phase relation is random (i.e. when there is no phase relation). When this signal (actually two different optical signals with a stable phase relation) impinges in the photodetector, it carries out an optical envelop detection, and the obtained photocurrent is proportional to the power of the optical signal.

Effectively, if the complex electrical fields of the two monochromatic signals are:

$$
\begin{aligned}
& E_{1}(t)=a \cdot e^{j\left[2 \pi f_{0} t+\phi_{1}\right]} \\
& E_{2}(t)=a \cdot e^{j\left[2 \pi\left(f_{0}+f_{R F}\right) t+\phi_{2}\right]}
\end{aligned}
$$

where $a$ is the amplitude, $f_{0}$ is the central frequency (for example, $193 \mathrm{THz}$ for the third window) and $\phi_{1}$ and $\phi_{2}$ are the phases of both signals. The current at the photodetector output is:

$$
\begin{aligned}
I_{P D}(t) & =\mathfrak{R}\left[E_{1}(t)+E_{2}(t)\right] \cdot\left[E_{1}(t)+E_{2}(t)\right]^{*}= \\
& =2 \mathfrak{R} a^{2}\left[1+\cos \left(2 \pi f_{R F} t+\left(\phi_{1}-\phi_{2}\right)\right)\right]
\end{aligned}
$$

It can be seen that the RF component of the photocurrent exhibits a phase equal to the phase difference between both optical carriers. If one of the optical component ( $E_{l}$ for instance) is a data signal instead of a monochromatic carrier, the same behaviour occurs, resulting in a phase shifting induced in the data signal after photodetection, which depends on the phase of the other signals ( $E_{2}$ in this case).

Once we understand the process of inducing phase shifting by optical heterodyne beating (envelop detection), it is easy to see how the optical matrix can be used as phase shifter element in a heterodyne beamforming network system. In Figure 66, a very simplified scheme for such system using a $2 \times 2$ optical Butler matrix (2 input and 2 output ports) is shown.

In Figure 67, the spectra of the signals at the indicated points (A-F) are depicted schematically. 


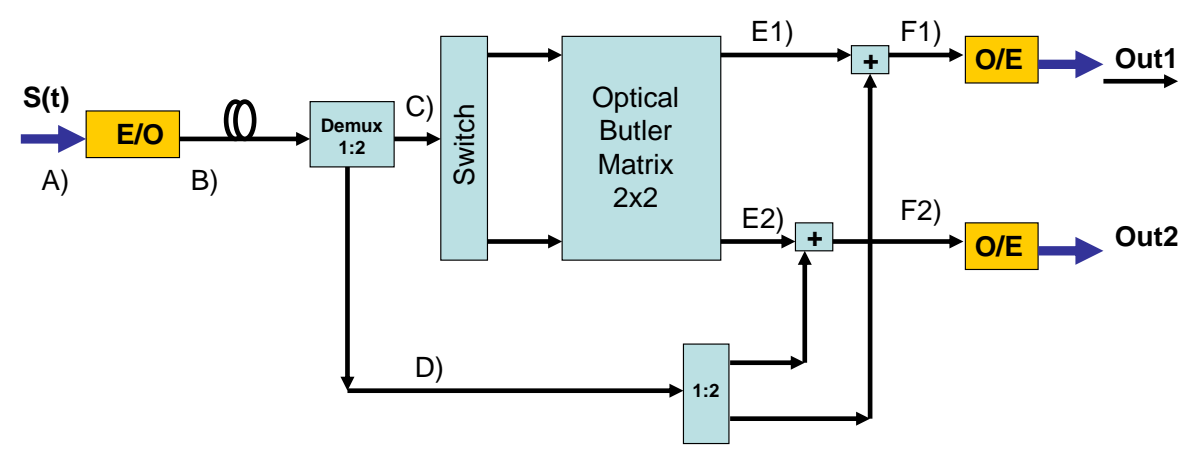

Figure 66. Optical B eamforming Network (OBFN) using a 2x2 optical B utler matrix.
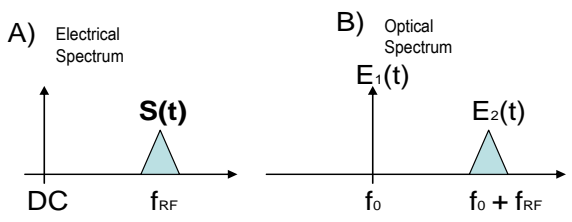

C) Optical

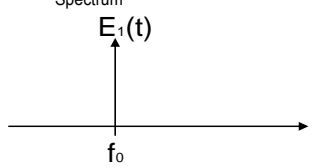

D) Optical
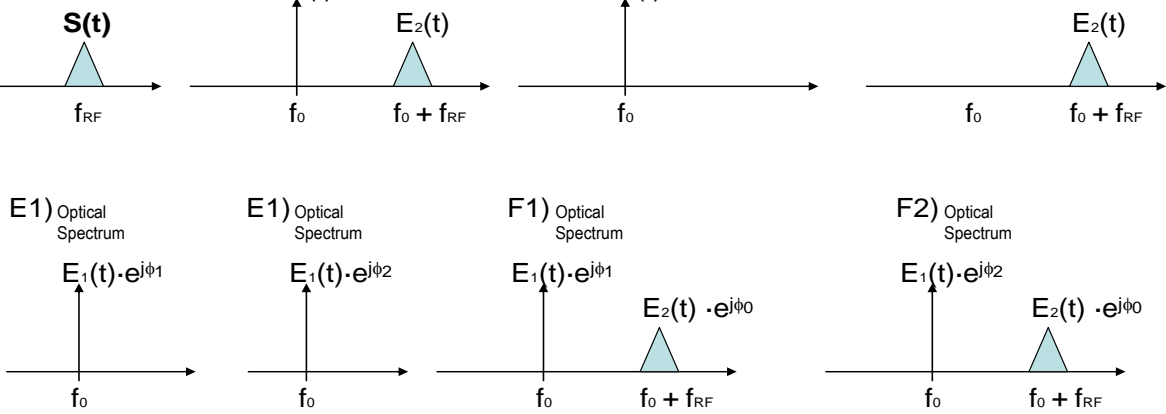

$$
\begin{aligned}
& \text { Out1 }=\mathbf{S}(\mathbf{t}) \cdot e^{\mathrm{j}\left(\phi_{1-} \phi_{0}\right)} \\
& \text { Out2 }=\mathbf{S}(\mathbf{t}) \cdot \mathrm{e}^{\mathrm{j}\left(\phi_{2-}-\phi_{0}\right)}
\end{aligned}
$$

Figure 67. Sche matic view of the signal s pectrum (both electrical and optical) at each of the indicated points in Figure 66.

The original signal to be transmitted by the antenna array is $S(t)$ (point A), allocated at $f_{R F}$. This signal modulates an optical carrier (single sideband modulation indicated in the figure for the sake of simplicity) with frequency $f_{0}$, resulting in a spectrum like the depicted in the Figure 67 (B). The two components $\left(E_{l}\right.$ being the optical carrier and $E_{2}$ the sideband) are separated in a 1:2 demux. The carrier is injected to an optical switch which routes the signal to one of the input ports of the Butler matrix (depending what beam direction is considered). This signal is replicated in the two output ports of the matrix with, in principle, the same amplitude in all, but with a differential phase, named phase profile, (points E1 and E2).

On the other side, the sideband is routed through a parallel path, named reference path, split in a 1:2 splitter (in general, $1: \mathrm{N}$, being $\mathrm{N}$ the number of ports of the 
matrix), and respectively combined with the carriers at the matrix output (F1 and F2), taking care in maintaining the same phase in all the sideband replicas $\left(\phi_{0}\right)$. After photodetection, as many replicas of the $S(t)$ signal as number of matrix point are generated with a phase profile similar to the one performed by the matrix in the optical field. Then, for this implementation, the number of beam directions obtained with a $\mathrm{NxN}$ optical Butler Matrix is equal to $\mathrm{N}$ (typically $\mathrm{N}=2^{\mathrm{n}}$ ).

\subsection{N Beam Directions with a NxN Optical Butler Matrix}

One of the simplest ways to duplicate the number of beam directions with a similar scheme to the one described in Figure 68 is the proposed in [MAD02] which consists in including an additional switch that enables to route the reference signal either through the reference path (like in Figure 66) or through one port of the matrix. This scheme is shown in the Figure 68, for the case of a $2 \times 2$ optical Butler Matrix.

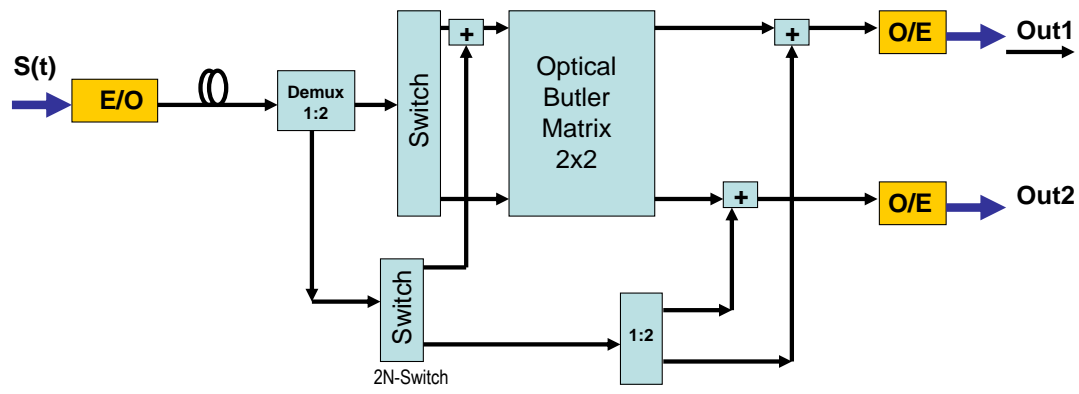

Figure 68. Scheme of the $2 \mathrm{~N}$ extension for the $\mathrm{OBFN}$.

When the sideband is routed through the reference path, it is combined with the signals at the output of the matrix with the same phase, as abovementioned. However, when it is routed through the matrix, both the optical carrier $\left(E_{l}\right)$ and the sideband $\left(E_{2}\right)$ exhibits a phase profile, and the resulting phase profile at the $\mathrm{O} / \mathrm{E}$ outputs is the difference of the $E_{1}$ and $E_{2}$ phases. If the sideband is injected at the same input port of the matrix (port number \#1 in the Figure 68), we can obtain as many beam directions as in the case of using the reference path. At the end, we obtain the same beam set as with the original scheme (Figure 66) and a set of beams with angular positions located in between the previously obtained as can be observed in the Figure 69 for an 8x8 matrix, where 16 beams are obtained. This can be achieved by injecting the sideband in any input port, not only for the first one. 


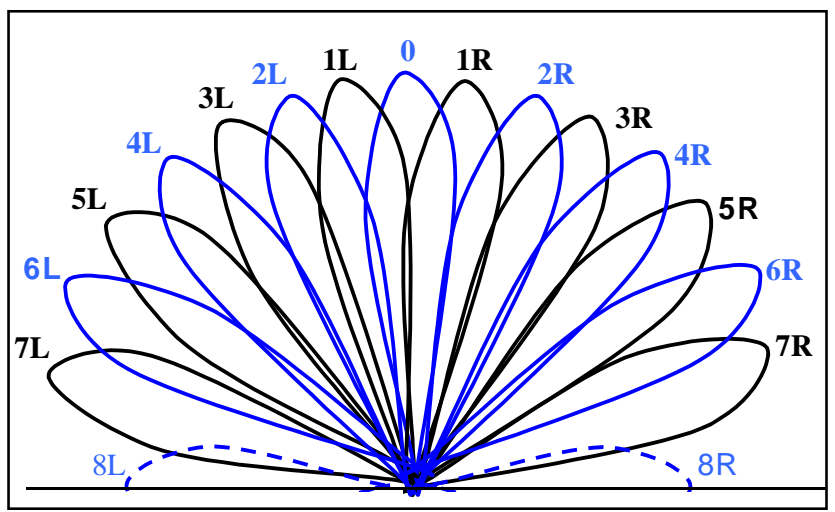

Figure 69. Optical Beamforming (OBF) using a $8 \times 8$ optical B utler matrix and Traditional $+2 \mathrm{~N}$ architecture.

\subsection{Design of an Integrated Optical Butler Matrix}

\subsubsection{The Optical Butler Matrix}

A schematic view of the integrated Butler Matrix indicating its main building blocks and a table summarizing the expected optical attenuation for the blocks is depicted in Figure 70. The main building blocks are the 90 degrees directional couplers, the phase shifters and the waveguides connecting these blocks. Additionally, since several waveguide crosses must be included in real implementations, they have been considered as building blocks too, and studied below. On the other hand, we can appreciate that, due to the attenuation of the waveguide crosses, a non-uniform power distribution is obtained at the output of the matrix. In order to mitigate this effect, also additional optical attenuations will be considered as another building block. For this case, extra-attenuation will be included with waveguide bends because the attenuation can be precisely tuned selecting the corresponding bend radii. This characterization is included in the corresponding section.

In principle, we will consider that any power non-uniformity at the output of the matrix will be corrected by the electrical amplification stage included at the antenna panel input. Then, the better and worse cases shown in the table from Figure 70 are related to the minimum and maximum attenuation introduced by the Butler Matrix between input-to-output ports, considering all the combinations with the same attenuation. 


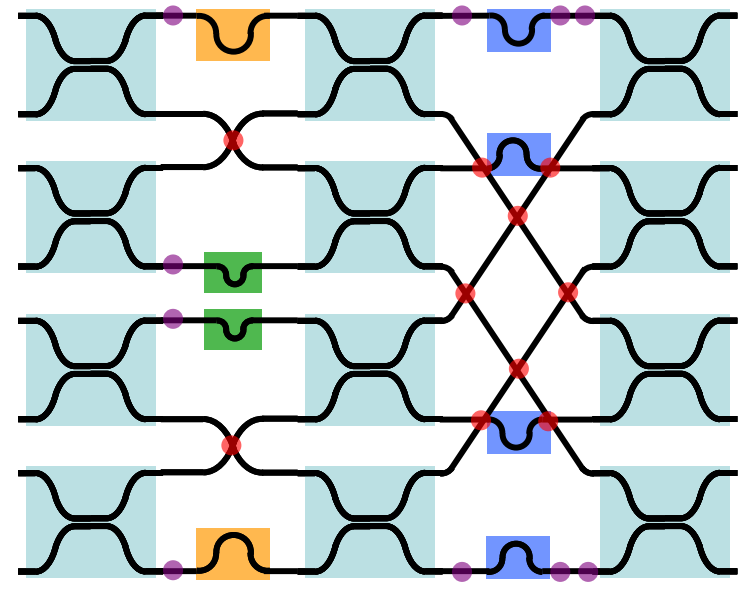

(a)

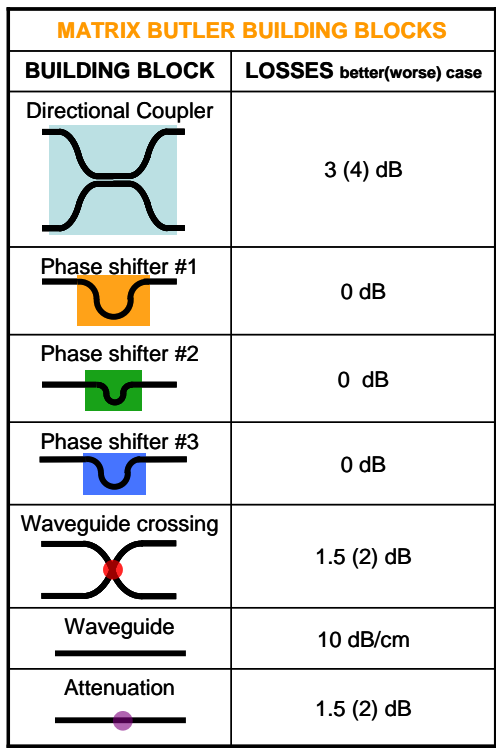

(b)

Figure 70. Sche matic view of the Butler Matrix (a) the Building Blocks' expected optical attenuation (b).

\section{Optical waveguides}

Optical waveguides are the basic element that interconnects the different building blocks in the Butler matrix. The main degradation in optical waveguides is propagation losses. The dominant source of propagation losses is sidewall roughness. Sidewall roughness is usually originated due to a non optimum fabrication process and can be modelled as a random variation of the waveguide width $^{10}$.
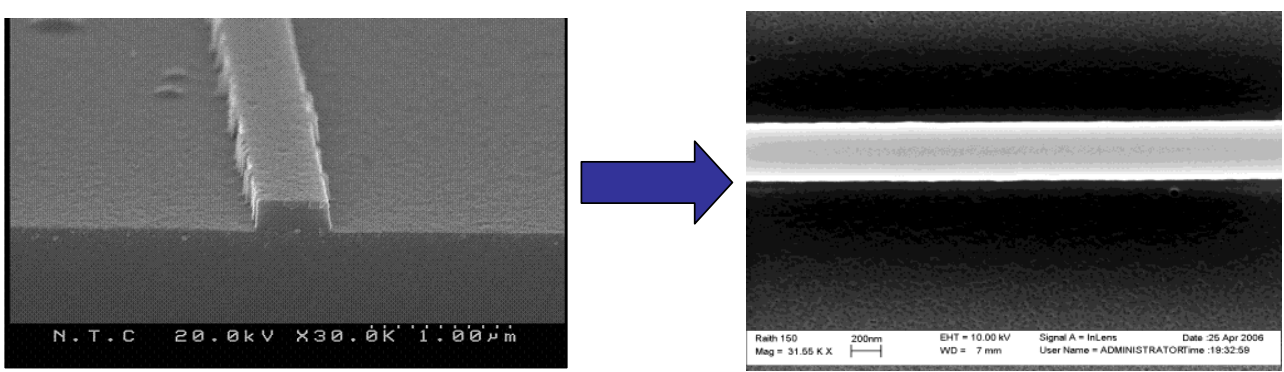

Figure 71. SEM images of two diffe rent wave guides. It can be seen the reduction of the side wall roughness.

10 See Annex B. 
Propagation losses lower than $15 \mathrm{~dB} / \mathrm{cm}$ have been obtained for the operation wavelength of $1550 \mathrm{~nm}$. The fabrication process based on lift-off was optimized in order to reduce sidewall roughness (Annex B). Some of the actions carried out were: optimization of the metal deposition, improvement of the ionic etching of the metal mask or the introduction of a plasma cleaning process in the silic on etching. Figure 71 shows SEM images of two different waveguides fabricated before and after optimization of the fabrication process. It can be seen that sidewall roughness have been significantly reduced. Sidewall roughness minimization gave rise to a decrease of the initial propagation losses of around $30 \mathrm{~dB} / \mathrm{cm}$ up to the currently propagation losses lower than $15 \mathrm{~dB} / \mathrm{cm}$.

\section{Bends}

Bend losses for different radii $(1 \mu \mathrm{m}, 2 \mu \mathrm{m}$ and $4 \mu \mathrm{m})$ have been experimentally evaluated to analyse the influence of sidewall roughness. As bend losses are rather low, several structures with waveguides containing a large number of bends were fabricated. Figure 72 shows SEM images of the reference waveguide structures and the waveguide with bends structures, and a detailed view of the bend structures with different radii. The obtained additional losses for bend radius of $5 \mu \mathrm{m}$ were lower than $0.1 \mathrm{~dB} /$ bend for a 180 degrees turn respect to a straight waveguide.
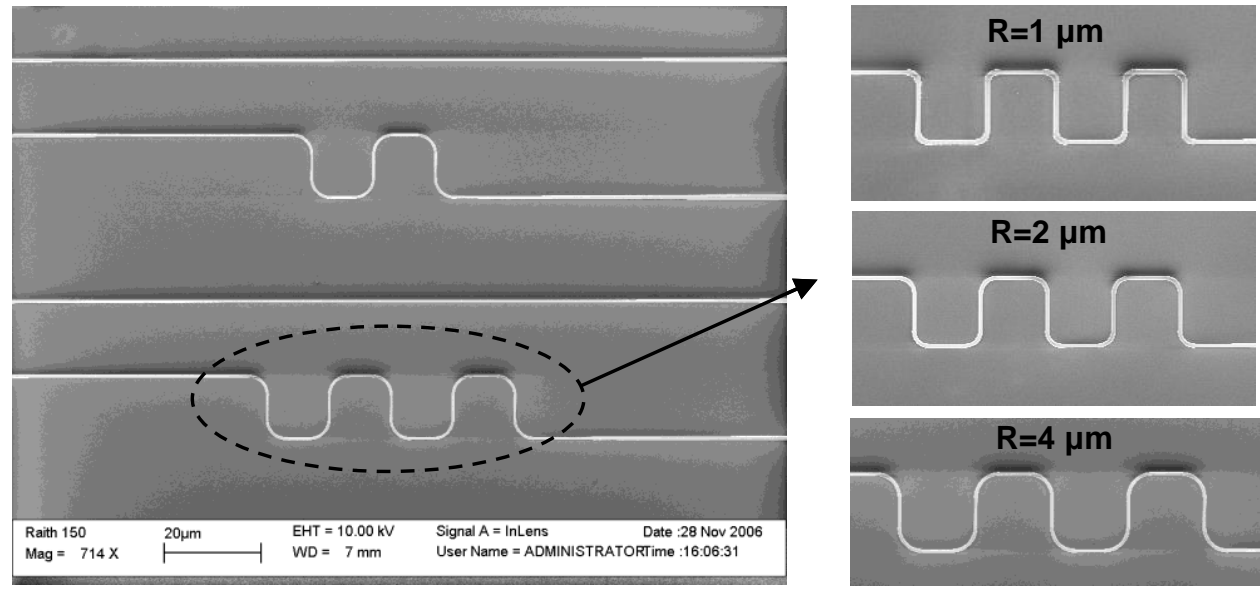

Figure 72. SEM images of the reference wave guide structures and the wave guide with bends structures and a de tailed view of the bend structures with diffe rent radii.

\section{$\underline{\text { Waveguides crosses }}$}

Waveguides crosses were also designed by means of 2D and 3D-FDTD simulations. Transmission losses, reflection losses and crosstalk losses were analysed for different crossing angles. Simulation results showed that although transmission losses are similar $\left(\mathrm{L}_{\mathrm{T}} \approx 1.5 \mathrm{~dB}\right)$ when the angle is between 60 and 
120 degrees, crosstalk losses are worse for 90 degrees $\left(\mathrm{L}_{\mathrm{CT}} \approx 12 \mathrm{~dB}\right)$ with respect to the optimum cases of 60 and 120 degrees $\left(\mathrm{L}_{\mathrm{CT}} \approx 22 \mathrm{~dB}\right)$. Crosstalk is higher when the angle is different from 60 and 120 degrees due to the higher mode mismatching at the crossing point. Figure 73 shows a SEM image of a waveguides cross with an angle of 90 degrees and SEM images of the crossing point at the different angles considered.

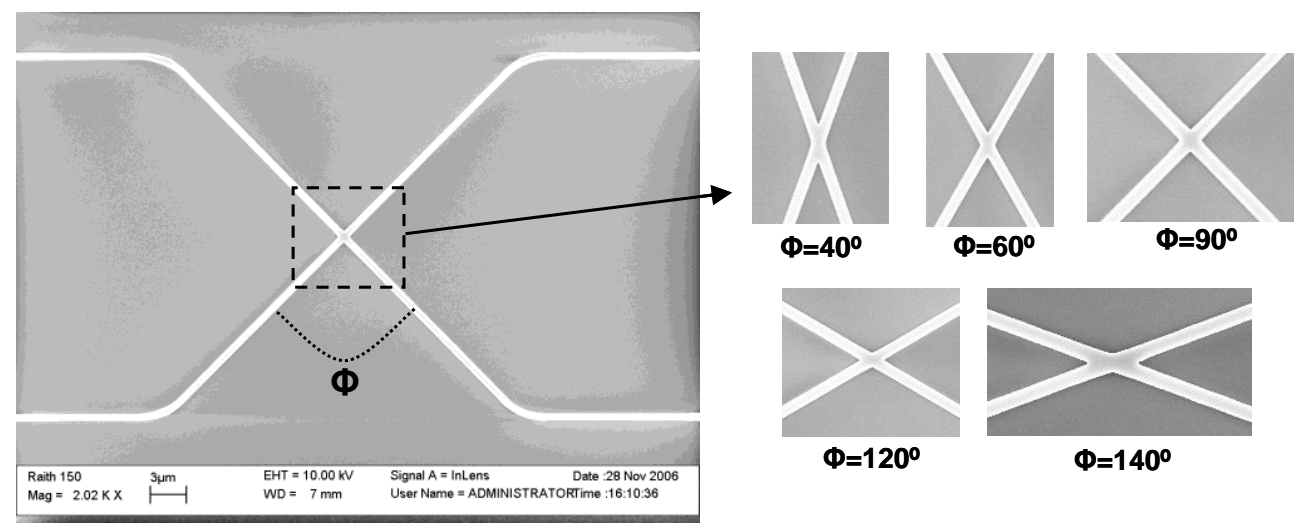

Figure 73. SEM image of a wave guides cross with an angle of 90 de grees and SEM images of the crossing point at the differe rent angles considered.

Figure 74 shows the experimental and 2D and 3D-FDTD simulated crosstalk losses as a function of the crossing angle. It can be seen that there is a good agreement between experimental and simulation results for the crossing angles. Furthermore, it is confirmed that crosstalk losses are higher for 90 degrees with respect to the optimum crossing angle of 60 degrees. The larger disagree for the crossing angles of 120 and 140 degrees are due to a non-optimum way of exposing in the lithography process. This effect is translated in higher waveguide widths for the four bends of the structure as shown in the SEM image of Figure 73 thus degrading the performance. The crossing angle of 60 degrees has been chosen as the optimum to implement the Butler matrix. Experimental crosstalk losses of $21.7 \pm 5.1 \mathrm{~dB}$ and insertion losses of $3.3 \pm 1.3 \mathrm{~dB}$ have been demonstrated for this angle. 


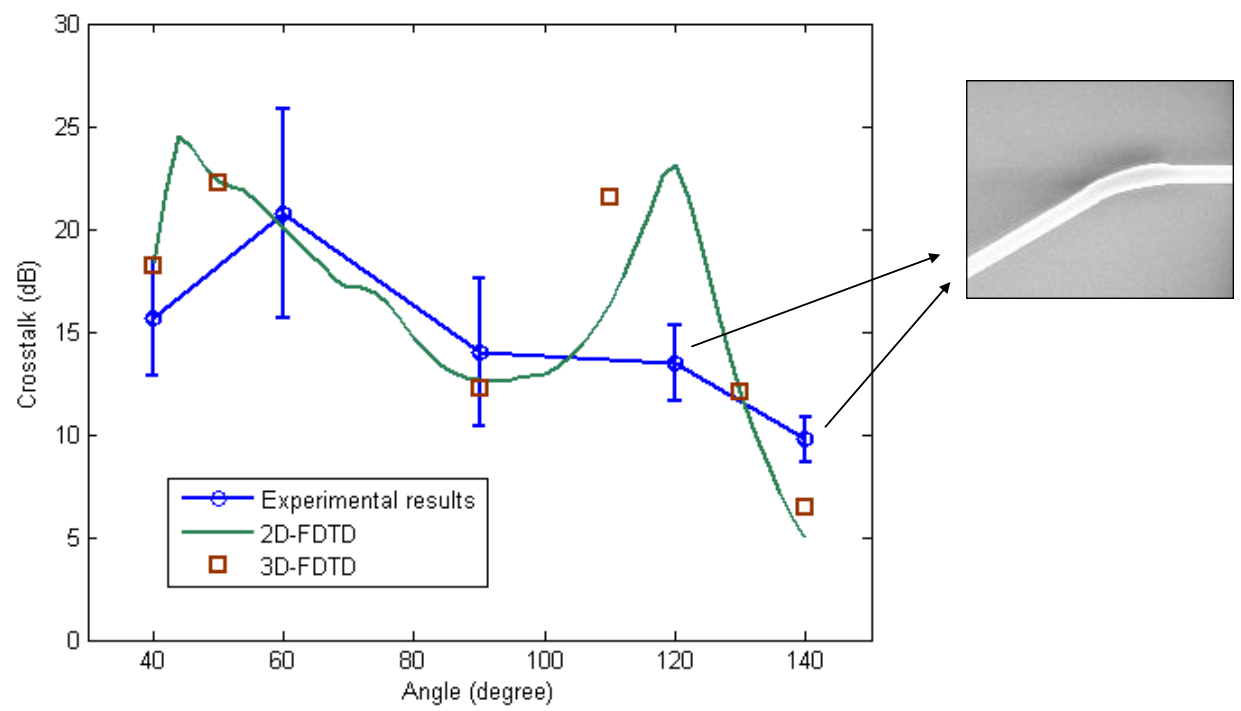

Figure 74. Experimental and 2D and 3D-FDTD simulated crosstalk losses as a function of the crossing angle.

\section{$\underline{\text { Couplers }}$}

Directional couplers as well as phase shifters are key elements in the Butler matrix. Therefore, significant efforts were carried out to optimize the fabrication process and characterize the influence of design parameters deviations on the building blocks performance. The parameters of the coupler chosen to optimize the fabrication process are $w=500 \mathrm{~nm}$ and $s=300 \mathrm{~nm}$. The coupler length was fixed to divide $50 \%$ of the input power through the output ports. The coupler structure was repeated several types in the same sample in order to evaluate the tolerance of the fabrication process. Figure 75 shows SEM images of fabricated couplers.

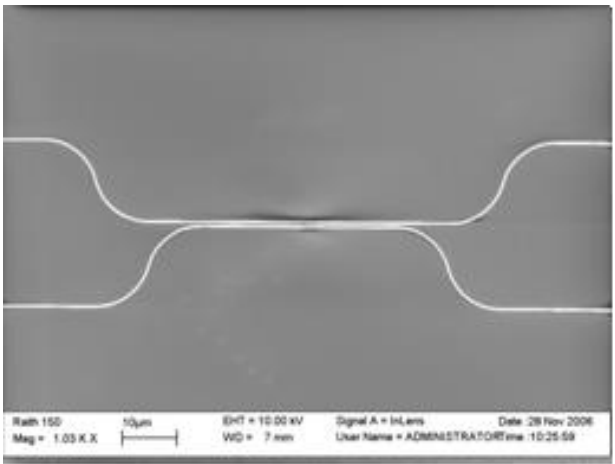

(up-left)

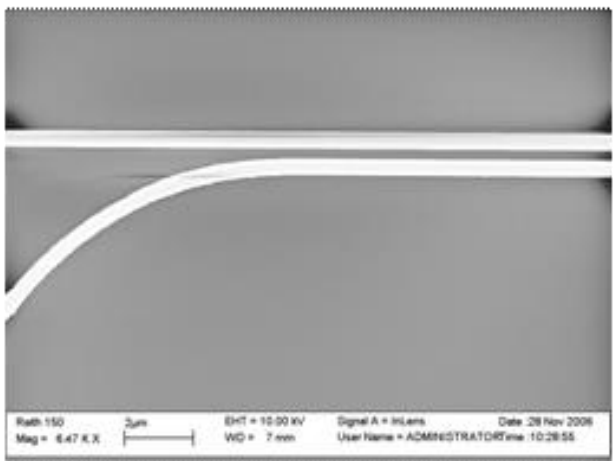

(up-right) 


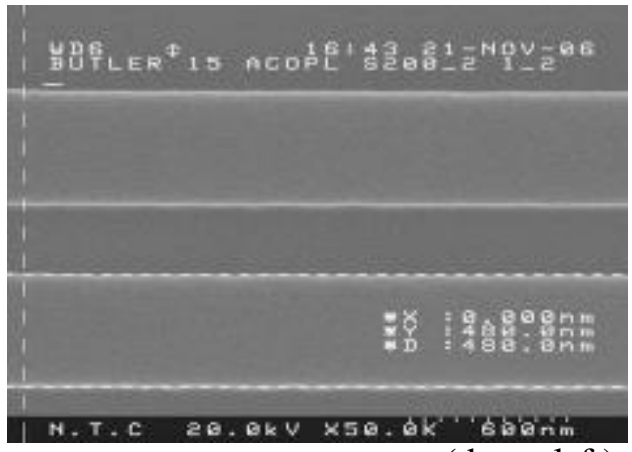

(down-left)

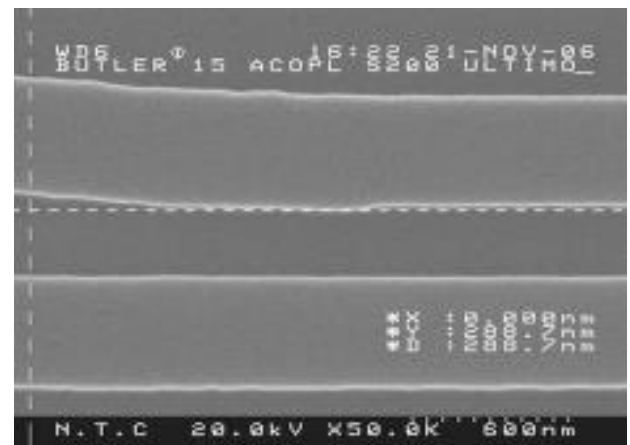

(down-right)

Figure 75: SEM images of fabricated couple rs (up). Detailed view of the wave guide separation in the middle of the coupler (down-left) and in the left extre me (down-right).

The waveguide width and waveguides separation of the fabricated structures were measured by SEM (Figure 75). In the fabrication, different e-beam dose (-10\%, standard dose (DN) and $+10 \%$ ) have been employed in order to characterize the tuning capability that can be obtained with different doses. The waveguide widths and separations have been measured by SEM observation (see Figure 75). In Table 11 , the mean and the standard deviation of the abovementioned measurements are depicted. For the standard dose, the fabricated waveguide width value is $478.1 \pm 5.2 \mathrm{~nm}$ while the mean waveguides separation value is $301.2 \pm 4.3 \mathrm{~nm}$.

\section{TABLE 11: STATIS TICAL ANALYS IS OF THE MEAS UREMENTS. B ETTER RESULTS ARE OBTAINED FOR LOWER DOSES WHICH EXHIBITS LOWER PROXIMITY EFFECTS.}

Statistical Analysis
\begin{tabular}{|c|c|c|c|}
\hline $\begin{array}{c}\text { E-beam } \\
\text { Dose }\end{array}$ & Statistical Parameter & $\begin{array}{c}\text { Waveguides } \\
\text { Width (nm) }\end{array}$ & $\begin{array}{c}\text { Waveguides } \\
\text { Separation } \\
(\mathbf{n m})\end{array}$ \\
\hline $10 \%$ & MEAN & 483,8 & 295,0 \\
DN & & $\mathbf{4 7 8 , 1}$ & $\mathbf{3 0 1 , 2}$ \\
$-10 \%$ & 471,9 & 310,0 \\
$10 \%$ & STANDARD DEVIATION & 3,4 & \\
DN & $\mathbf{5 , 2}$ & $\mathbf{2 , 7}$ \\
$-10 \%$ & & 2,7 & 4,3 \\
\hline
\end{tabular}

Taking these values into account, the length of the directional coupler could be recalculated to achieve $50 \%$ power division. However, for the Butler matrix performance, it is not critical to achieve a perfect power division as deviations even much higher than the obtained will only suppose a small unbalancing in $\mathrm{dB}$ between 
both output ports (for instance a $60 \% / 40 \%$ distribution implies a $-2.2 \mathrm{~dB} /-4.0 \mathrm{~dB}$ distribution, so the power is unbalanced by less than $2 \mathrm{~dB}$ between the two output ports, and a deviation of approximately $1 \mathrm{~dB}$ from the ideal $-3 \mathrm{~dB} /-3 \mathrm{~dB}$ case). Furthermore, the most important function of the coupler in the Butler matrix is to achieve a 90 degrees phase shift between both output ports which is always achieved independently of the power coupling distribution between both output ports.

\section{Phase shifters}

The use of a Mach-Zehnder interferometer (MZI) has been used in order to estimate the phase shift. Interferometer structures convert phase differences in power transmission variations. The length of one of the MZI arms is modified to achieve the phase shifts of 22.5, 45 and 67.5 degrees. An additional length difference $(\Delta \mathrm{L}=13.464 \mu \mathrm{m})$ is considered in the MZI structure to achieve a minimum of transmission at $1550 \mathrm{~nm}$. Thus, the introduction of the phase shifts is carried out by increasing the $\Delta \mathrm{L}$ length which shifts the minimum of transmission to higher wavelengths. Figure 76 shows a SEM image of one of the fabricated MZI structures with directional couplers.

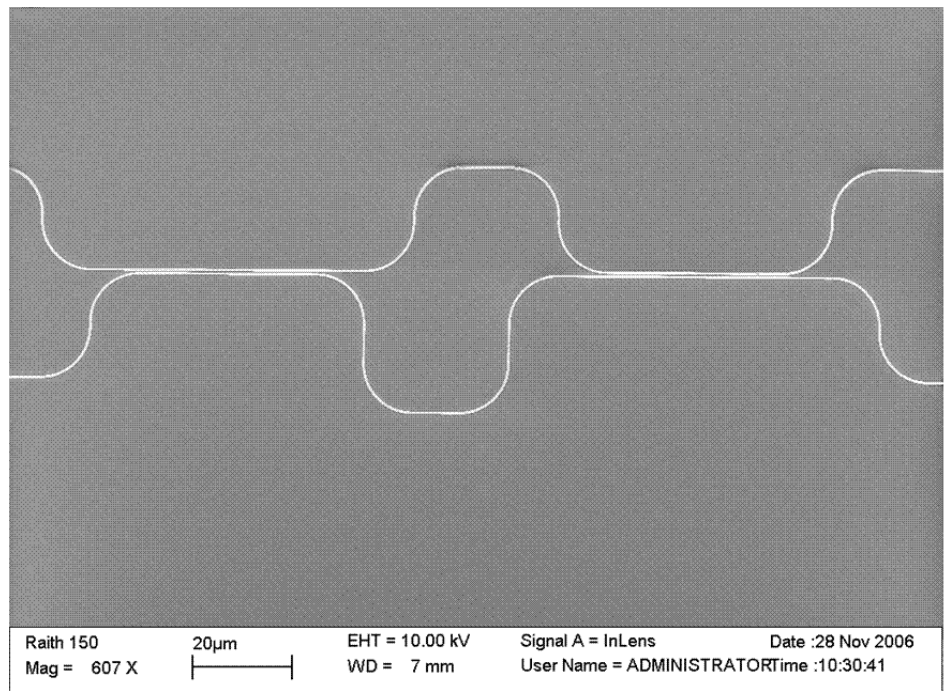

Figure 76. SEM image of a fabricated MZI s tructure with dire ctional couplers.

Figure 77 shows the experimental and simulated results of the transmitted power as a function of the wavelength for the cases of (a) reference structure, (b) 22.5 degrees, (c) 45 degrees and (d) 67.5 degrees. As before, the waveguide width variation was evaluated by means of SEM inspection and the obtained data used to simulate the MZI structures. It can be seen that experimental and simulated results show and reasonable agreement thus indicating that precise control of the 
required distance is achieved. It should be noticed that the difference in length between the MZI structures is of only around $40 \mathrm{~nm}$. The precise control of the required distance was also confirmed by measuring MZI structures with the same length in both arms.
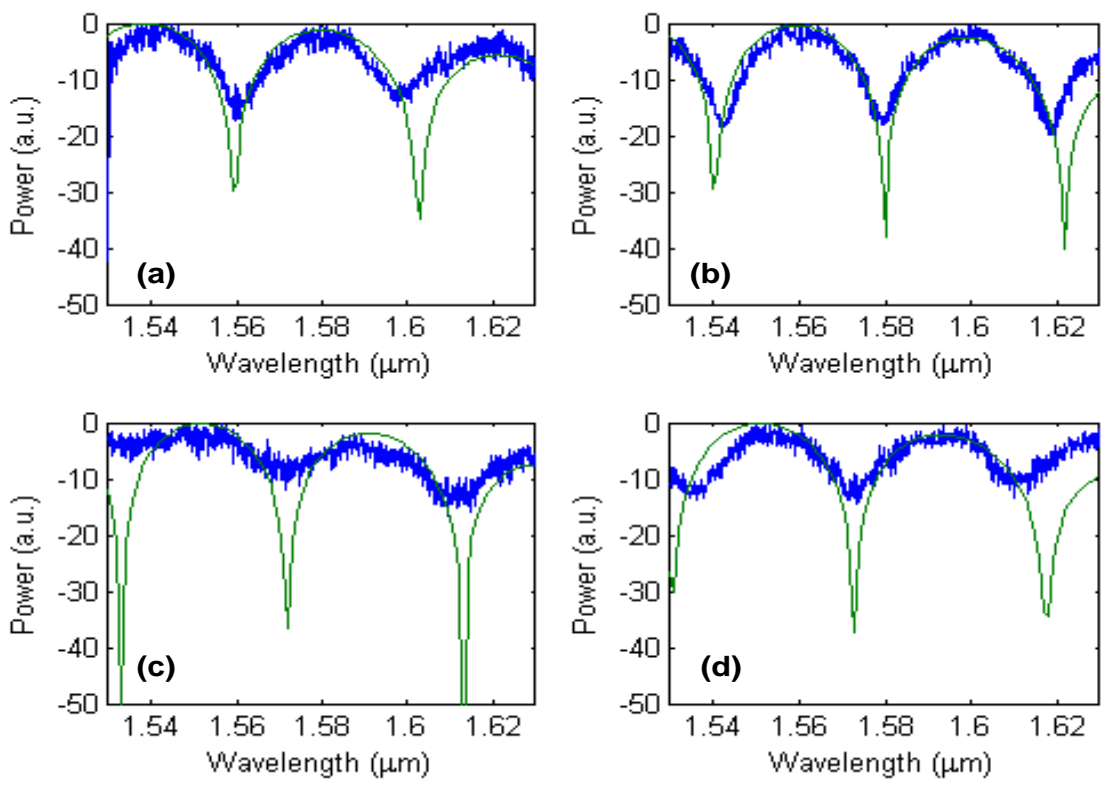

Figure 77. Experimental and simulated results of the trans mitted power as a function of the wavelength for the cases of (a) refe re nce structure, (b)

22.5 degrees, (c) 45 degrees and (d) 67.5 degrees. 
TABLE 12: SUMMARY OF THE FAB RIC ATED BUILDING BLO CKS AND EFFEC T IN THE RADIATIO N DIAGRAM

\begin{tabular}{|c|c|c|c|c|c|}
\hline $\begin{array}{c}\text { Building } \\
\text { Block } \\
\text { Description } \\
\end{array}$ & $\begin{array}{l}\text { Schematic } \\
\text { View }\end{array}$ & SEM pictures & $\begin{array}{l}\text { Theoretical } \\
\text { Parameters }\end{array}$ & Measured Parameters & $\begin{array}{c}\text { Effect in the } \\
\text { Radiation Pattern } \\
\text { Diagram (1) } \\
\end{array}$ \\
\hline $\begin{array}{c}\text { Optical } \\
\text { Waveguide }\end{array}$ & Waveguide & & $L_{\text {prop }}=10 \mathrm{~dB} / \mathrm{cm}$ & $L_{\text {prop }}=15 \mathrm{~dB} / \mathrm{cm}$ & $\begin{array}{l}\text { Negligible additional } \\
\text { optical losses }\end{array}$ \\
\hline Bend & & & $L_{b e n d}<1 d B$ & $\begin{array}{l}L_{b e n d}(1 \mu \mathrm{m})=0.33 \pm 0.14 \mathrm{~dB} \\
L_{b e n d}(2 \mu \mathrm{m})=0.15 \pm 0.10 \mathrm{~dB} \\
L_{b e n d}(4 \mu \mathrm{m})=0.12 \pm 0.04 \mathrm{~dB}\end{array}$ & $\begin{array}{l}\text { Negligible additional } \\
\text { optical losses }\end{array}$ \\
\hline Cross & & & $\begin{array}{l}I L<2 d B \\
\chi>15 d B\end{array}$ & $\begin{array}{l}\chi\left(60^{\circ}\right)=21.7 \pm 5.1 \mathrm{~dB} \\
I L\left(60^{\circ}\right)=3.3 \pm 1.3 \mathrm{~dB}\end{array}$ & $\begin{array}{l}\text { Crosstalk: Negligible } \\
\text { effect in the radiation } \\
\text { diagram when } \\
\text { compared with other } \\
\text { sources of } \\
\text { degradation. } \\
\text { Insertion Losses: } \\
\text { Additional losses of } \\
1.3 \mathrm{~dB} \pm 1.3 \mathrm{~dB}\end{array}$ \\
\hline Coupler & & & $\begin{array}{l}\text { - Amplitude } \\
\text { Imbalance }<2.5 \mathrm{~dB} \\
- \text { Additional Losses } \\
<1.5 \mathrm{~dB} \\
- \text { Phase } \\
\text { Imbalance }<5^{\circ}\end{array}$ & $\begin{array}{l}\text { - Amplitude } \\
\text { Imbalance }<2 \text { dB } \\
\text { - Additional } \\
\text { Losses }<1 \text { dB } \\
\text { - Phase Imbalance }<5^{\circ}\end{array}$ & $\begin{array}{l}\text { - Residual sidelobe } \\
\text { level }<-22 \mathrm{~dB} \\
\text { - Directivity } \\
\text { degradation }<0.1 \mathrm{~dB}\end{array}$ \\
\hline
\end{tabular}




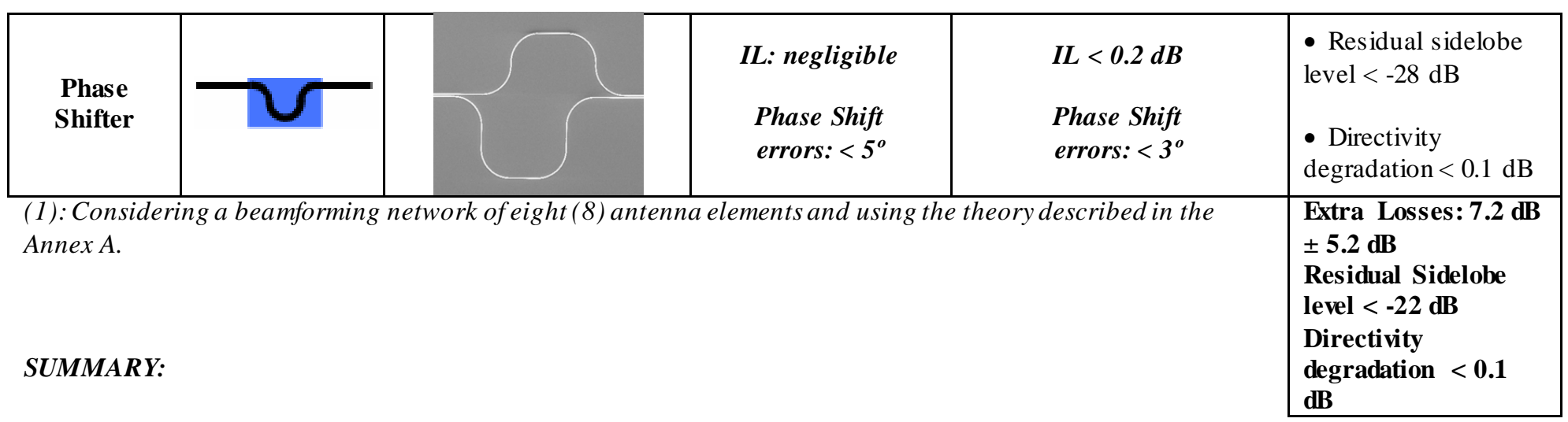




\section{5. $8 \times 8$ Butler Matrix Fabrication ${ }^{11}$}

The fabrication of the $8 \times 8$ Butler matrix was carried out by electron-beam lithography and reactive ion etching with inductively coupled plasma etching. A negative resist was used to obtain a better resolution and lower roughness [SAN06]. Two types of configurations were designed and fabricated, one to measure the output power performance and the other one to measure the phase shift performance. The former was simply created by conveniently enlarging both the eight input and output ports to the chip facets. The latter was fabricated as shown in Figure 78.
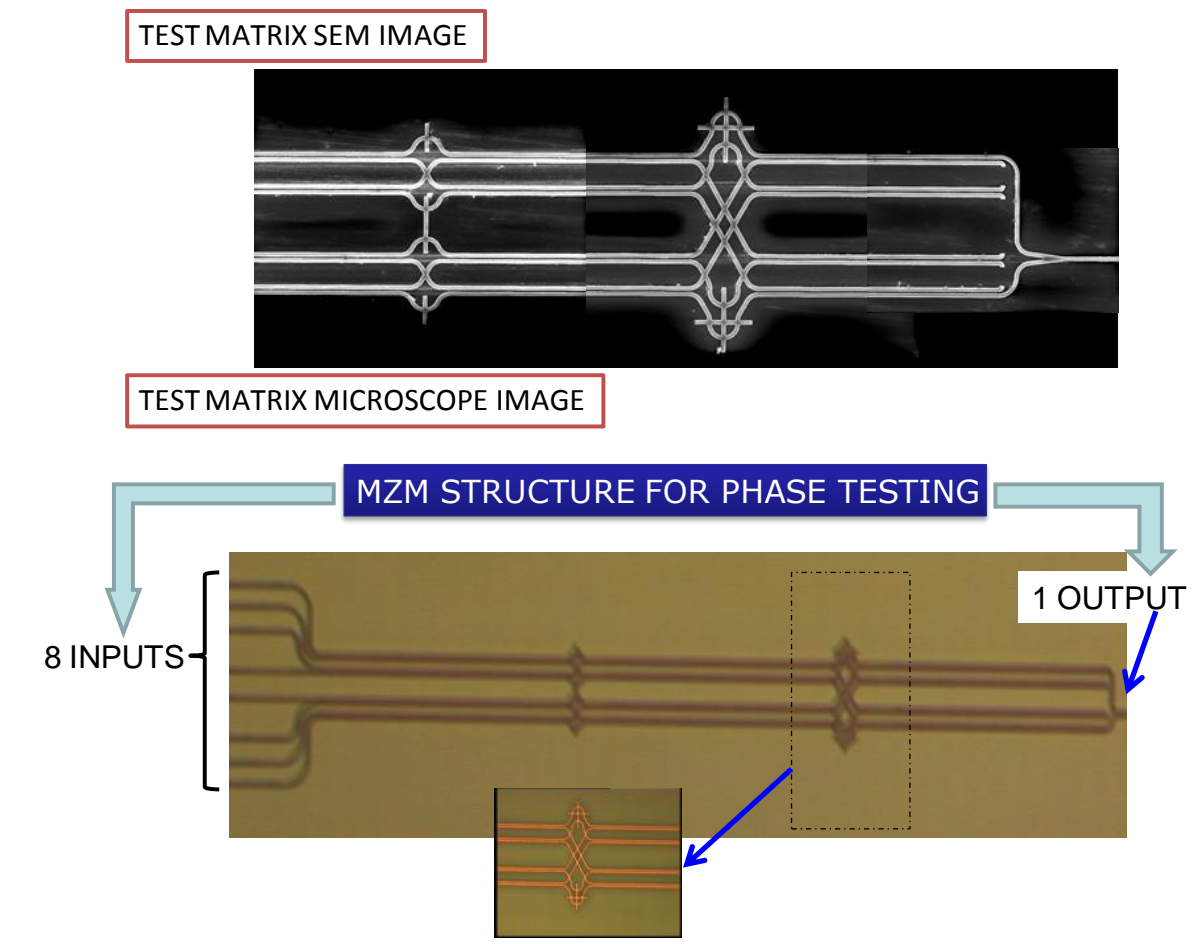

Figure 78. SEM and optical microscope images of the fabricated 8x8 Butler matrix.

Figure 78Figure 78. SEM and optical microscope images of the fabricated $8 \times 8$ Butler matrix. shows SEM and optical microscope images of the fabricated $8 \times 8$ Butler matrix to evaluate the phase shift performance. The worst case for the phase shift error between output ports occurs between the two output ports which are more separated. Therefore, the two extreme output ports of the matrix have been combined by using a Y-junction, as can be seen in Figure 78. In this way, the 8x8 Butler matrix

${ }^{11}$ Further details are provided in Annex B. 
is seen as set of eight 1:1 Mach-Zehnder interferometers so the phase shift performance introduced by the matrix, which depends on the selected input port, is transformed into a power modulation.

The lengths of the extra waveguide sections used to combine the two output ports of the matrix were properly designed and chosen to have a notch around $1550 \mathrm{~nm}$ in the spectral MZI response. Thus, the whole matrix can be evaluated by successively injecting light through each one of the input ports and measuring the spectral response of the virtual MZI structure.

\subsection{Characterization of the $8 \times 8$ Butler Matrix}

\subsubsection{Optical Insertion Losses}

Table 13 shows the output power measurements and the standard deviation of the output power variation. The total power in the eight output ports is also depicted. Ideally, output power values should be the same independently of the input port because the number of waveguide crosses, which is the main attenuation factor, is the same in all the possible paths of the Butler matrix. However, it can be seen that this does not occur.

TABLE 13: MEASURED OUTPUT POWER IN DBM AS A FUNCTION OF THE INPUT PORT. THE LAS T COLUMNS SHOW THE TOTAL POWER IN THE EIGHT OUTPUT PORTS AND THE STANDARD DEVIATION OF THE OUTPUT POWER VARIATION.

\begin{tabular}{|c|c|c|c|c|c|c|c|c|c|c|}
\hline & Out 1 & Out 2 & Out 3 & Out 4 & Out 5 & Out 6 & Out 7 & Out 8 & $\begin{array}{c}\text { Total } \\
\text { Power } \\
\text { (dlBm) }\end{array}$ & $\begin{array}{c}\text { op } \\
(\mathbf{d B B})\end{array}$ \\
\hline In 1 & $-46,3$ & $-48,3$ & $-48,9$ & $-47,8$ & $-47,2$ & $-45,1$ & $-50,3$ & $-50,4$ & $-38,7$ & 1,8 \\
\hline In 2 & $-47,3$ & -51 & $-46,7$ & $-46,4$ & $-48,8$ & $-49,2$ & $-49,4$ & -49 & $-39,2$ & 1,6 \\
\hline In 3 & $-47,1$ & $-48,8$ & $-47,9$ & $-47,5$ & $-50,6$ & $-50,3$ & $-51,2$ & $-50,6$ & $-40,0$ & 1,6 \\
\hline In 4 & $-47,4$ & $-45,7$ & $-48,7$ & $-46,7$ & $-47,5$ & $-47,6$ & $-50,6$ & $-51,1$ & $-38,8$ & 1,9 \\
\hline In 5 & $-49,6$ & $-47,9$ & -43 & $-44,3$ & $-47,3$ & $-49,1$ & $-53,9$ & $-53,3$ & $-38,1$ & 3,8 \\
\hline In 6 & $-51,3$ & $-50,8$ & $-50,5$ & $-49,5$ & $-50,9$ & $-50,1$ & $-52,8$ & $-52,9$ & $-41,9$ & 1,2 \\
\hline In 7 & $-47,9$ & $-45,7$ & $-52,5$ & $-51,8$ & $-47,4$ & -51 & $-44,5$ & -46 & $-38,5$ & 3,0 \\
\hline In 8 & $-47,6$ & $-45,7$ & $-53,5$ & $-53,9$ & $-47,7$ & $-51,5$ & $-47,2$ & $-48,6$ & $-39,6$ & 3,1 \\
\hline
\end{tabular}

Firstly, the total power values shown in Table 13 have been compared with the output power of a straight $3 \mu \mathrm{m}$ wide waveguide to analyse the insertion losses of the $8 \times 8$ Butler matrix. The output power measured in a $3 \mu \mathrm{m}$ wide waveguide was $-31.3 \mathrm{dBm}$. Thus, the difference between this value and the values of the total power at the output of the Butler matrix is around $7 \mathrm{~dB}$ for all the input ports. These losses are mainly due to the transmission losses of the waveguide crosses. Eachcross 
has around $1.6 \mathrm{~dB}$ transmission losses so the total losses due to the four waveguide crosses will be $6.5 \mathrm{~dB}$ which is in very good agreement with the previous value. Therefore, it can be concluded that the insertion losses in the 8x8 Butler matrix are mainly determined by the waveguide crosses.

\subsubsection{Phase Shift Performance of a $8 \times 8$ Butler Matrix}

As previously explained the phase shift performance can be evaluated by measuring the spectral response of the virtual MZI structure introduced in the 8x8 Butler Matrix shown in Figure 78. Figure 79 shows the experimental and simulated results of the normalized transmitted power as a function of the wavelength. The title of each plot describes the input port.

In Figure 79, it has been included the frequency response measurements for the eight ports of the studied sample, the theoretical response to be obtained according with the designed physical parameters (effective refraction index, waveguides length, couplers, crosses, etc.), and the response deviation considering an absolute phase error due to phase shift standard deviation $\left(\sigma_{\varphi}\right)$ of $5 \mathrm{~nm}$, and recalculating the error for the exact length of the branches of the $8 \mathrm{MZ}$ interferometers. The unbalance of the coupling coefficient of the couplers has also been considered in the calculations as well as an estimation of the coupling frequency response. It is worth nothing to say that the free spectral range (FSR) (frequency separation between adjacent nulls of the MZI response) increases progressively with the selected input port. Then, the lower FSR, the lower input port number is. This is the reason why the null of the first figures are clearer than the others.

As can be seen in the figure, the measured response fits in between the depicted theoretical responses, which covers the $99.72 \%$ of the cases ( $3 \sigma$ criterion). In the following table, a comparison between the expected phase differences at the output of the matrix between the output ports \#1 and \#8 and the obtained with the approximation is depicted. Also the standard deviation of the phase errors at the output of the port \#1 (similar to the port \#8) for all the inputs is depicted. This value has been calculated with the model described above for the physical lengths of each of the optical path inside the $8 \times 8$ matrix. The obtained values are around 5 degrees. 

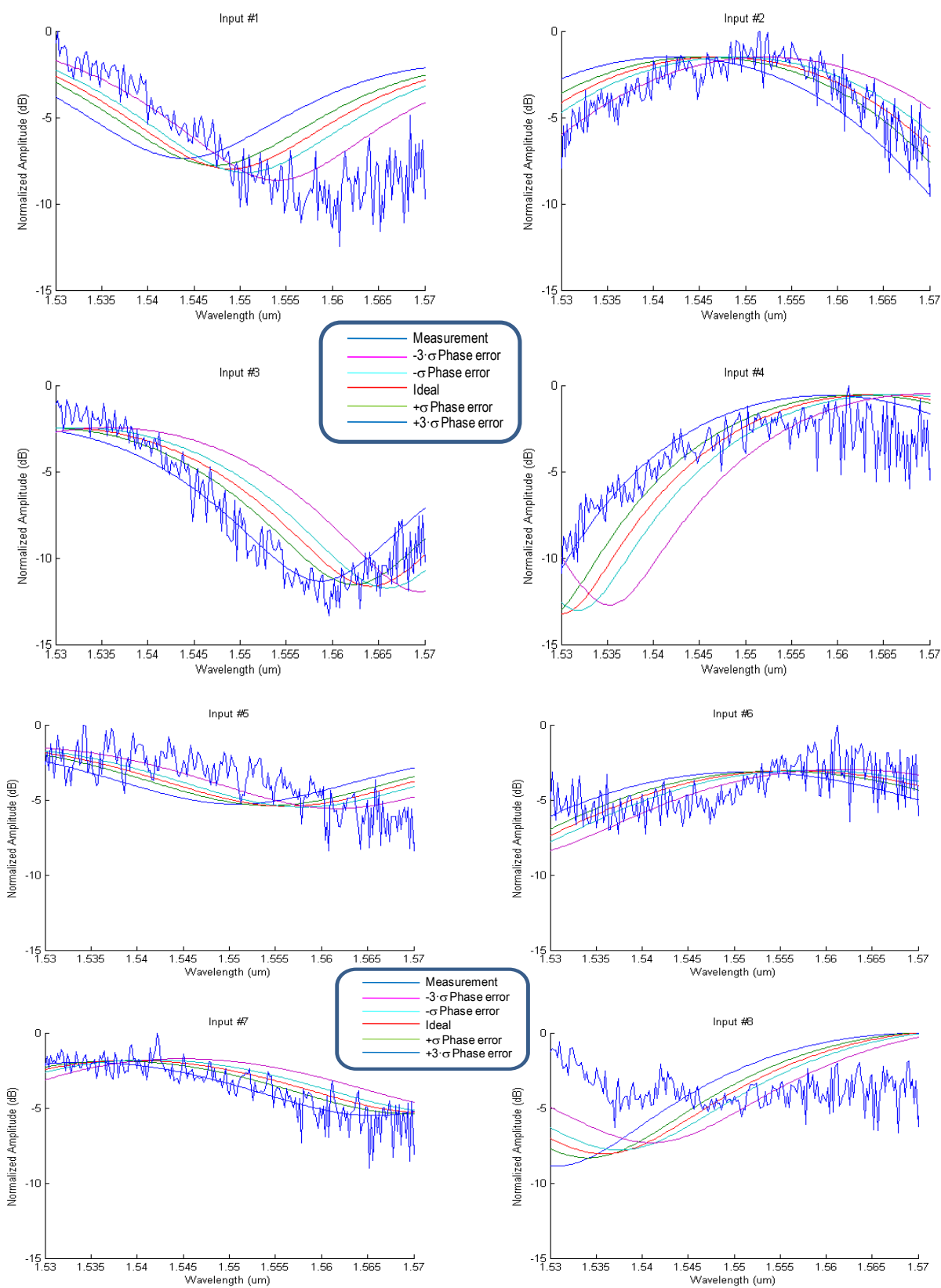

Figure 79. Experimental and simulated results of the normalize d trans mitted power as a function of the wavelength. The title of each plot describes the input port. 
TABLE 14: COMPARISON BETWEEN THE IDEAL AND MEASURED PHASE DIFFERENCE AND THE OBTAINED S TATIS TICAL DEVIATION OF THE PHASE S HIFT ERROR WHEN LIGHT IS INJECTEID THROUGH EACH ONE OF THE INPUT PORTS.

\begin{tabular}{|c|c|c|c|c|c|c|c|c|}
\hline & In1 & In2 & In3 & In4 & In5 & In6 & In7 & In8 \\
\hline $\begin{array}{c}\text { Ideal } \\
\Delta \Phi\end{array}$ & -157.5 & 22.5 & -67.5 & 112.5 & -112.5 & 67.5 & 22.5 & 157.5 \\
\hline $\begin{array}{c}\text { Meas } \\
\Delta \boldsymbol{\Phi}\end{array}$ & -155.775 & 24.2252 & -67.055 & 112.945 & -112.945 & 67.055 & -24.2252 & 155.775 \\
\hline $\boldsymbol{\sigma}_{\boldsymbol{\varphi}}$ & 5.038 & 5.038 & 5.026 & 5.026 & 5.022 & 5.022 & 5.018 & 5.018 \\
\hline
\end{tabular}

\subsection{Impact of experimental results on System Performance}

The following accuracy requirements for the phase and amplitude errors in the illumination function were considered according to the requirements in a typical active array antenna for the application scenario provided:

- $\sigma_{\phi}<10$ degrees

- $\sigma_{\mathrm{a}}<3 \mathrm{~dB}$

considering that all errors are included, even those from components placed at the input and output of the BFN for sideband extraction/re-combining; and that a further analysis is performed on the effects of BFN errors.

The following values have been obtained in the matrix evaluation according the previous section, issued from processing the full BM test results to estimate actual splitting ratios from the couplers. The phase errors have been estimated as explained in previous sections from waveguide roughness and width. The values for the $8 \times 16$ and the whole beamformer have been calculated as described in the Annex A.

\section{TABLE 15: ERROR BUdget CALCULATIONS FOR A COMPLETE BFN INCLUDING} 8X8 BM TEST-RESULTS

\begin{tabular}{|lcccc|} 
& $\begin{array}{c}\text { Calculation } \\
\text { Input }\end{array}$ & $\begin{array}{c}\text { Test } \\
\text { Results }\end{array}$ & Calculation & Calculation \\
$\begin{array}{c}\text { Couplers \& } \\
\text { phase shifters }\end{array}$ & $\begin{array}{c}\mathbf{8 x 8} \\
\text { Matrix }\end{array}$ & $\begin{array}{c}\mathbf{8 x 1 6} \\
\text { Matrix }\end{array}$ & $\begin{array}{c}\text { Whole } \\
\text { Beamformer (two } \\
\text { cascaded 8x16) }\end{array}$ \\
\hline Parameter & 3.80 & 5.04 & 6.33 & $\mathbf{8 . 9 5}\left(=\sigma^{\circ}\right)$ \\
\hline Phase $\left(^{\circ}\right)$ & 0.53 & 2.00 & 2.07 & $\mathbf{2 . 9 3}\left(=\sigma_{\mathrm{dB}}\right)$ \\
\hline Amplitude $(\mathrm{dB})$ & &
\end{tabular}


Then, we deduce the effects on the antenna radiation performances by using the abovementioned formulas and the results are shown next.

\section{TABLE 16: SUMMARY OF ERRORS AND THEIR EFFECT ON THE ANTENNA RADIATION PATTERN ${ }^{12}$}

\begin{tabular}{|c|c|c|c|c|c|c|}
\hline \multicolumn{2}{|c|}{$\begin{array}{c}\text { Normalised } \\
\text { amplitude errors }\end{array}$} & \multicolumn{2}{|c|}{$\begin{array}{c}\text { Normalised phase } \\
\text { errors }\end{array}$} & $\begin{array}{c}\text { Directivity } \\
\text { decrease }\end{array}$ & $\begin{array}{c}\text { Spurious } \\
\text { average } \\
\text { level }\end{array}$ & $\begin{array}{c}\text { Spurious } \\
\text { peak level } \\
(1 \% \text { prob) }\end{array}$ \\
\hline $\begin{array}{c}\Delta=\sigma_{\mathrm{dB}} \\
/ 8.68\end{array}$ & $\begin{array}{c}\Delta^{2} \\
(\text { variance) }\end{array}$ & $\delta=\sigma^{\circ} / 57.3$ & $\begin{array}{c}\delta^{2} \\
(\text { variance })\end{array}$ & $\begin{array}{c}\Delta \mathrm{D} \text { from } \\
(\mathrm{A} .3)\end{array}$ & $\begin{array}{c}\text { SL from } \\
(\mathrm{A} .4)\end{array}$ & $\mathrm{SL}+6.4 \mathrm{~dB}$ \\
\hline 0.337 & $\mathbf{0 . 1 1 3 8}$ & 0.156 & $\mathbf{0 . 0 2 4 4}$ & $-0.6 \mathrm{~dB}$ & $\begin{array}{c}-32.7 \\
\mathrm{~dB} / \mathrm{Max}\end{array}$ & $\begin{array}{c}-26.1 \\
\mathrm{~dB} / \mathrm{Max}\end{array}$ \\
\hline
\end{tabular}

Computations from the error budget deduced from the test results of the $8 \times 8$ Butler Matrix (removing some part of them coming from measurement, thanks to a 'retrofit' algorithm) have been done generalizing the amplitude and phase unbalances to the full Butler-based beamformer. As a result, the errors moderately impact the antenna performances (see Annex A):

- $\quad$ around $0.6 \mathrm{~dB}$ directivity decrease.

- the "C/I"

${ }^{12}$ All values are statistical averages in the table; but for simplification, the upper lines (such as in $\bar{\delta}$ ) have been left out.

${ }^{13}$ Normalised values, according to Annex A (A.6) allow to directly compare influence of amplitude versus phase errors; in Table 16 of Annex A, only overall values, for whole beamformer, are kept.

${ }^{14} \mathrm{C}$ is any carrier within the multiplex radiated in the nominal antenna lobe; $\mathrm{I}=\Sigma \mathrm{i}$ is the sum of all interfering carriers using the same frequency " 1 spot over 4" thanks to a 1:4 regular frequency-reuse pattern. 


\subsection{Distribution Input Matrix}

The previous sections have covered the work carried out to manufacture a pure optical Butler matrix, but this structure is not sufficient to implement the required phase shifting in the microwave signal. For doing so, an optical circuitry that performs the carrier to sideband separation is needed according with the process described in the section 5.2. In this section the research carried out for the characterization of the heterodyne generation of the RF phase distribution is included. For that purpose, a $4 \mathrm{x} 4$ photonic Butler Matrix together with a Distribution Input Matrix (DIM) structure has been designed and fabricated for the validation of the functionality in the electrical domain.

The design of the integrated photonic structure can be divided in two building blocks: The Distribution Input Matrix and the 4x4 Butler Matrix. The design of the Butler Matrix was already considered in the previous sections. On the other hand, a special DIM has been designed for the $4 \times 4$ Butler Matrix in order to prove the concept. The DIM consists on several filtering elements that split the sideband component of the optical field from the carrier. Then it guides each of the components to different inputs in order to obtain at the output the desired phase distribution. In the following sections, the design of the DIM and of its basic components is explained.

\subsubsection{DIM structures and sample characterization}

The DIM structure routes the carrier and the sidebands of the optical modulations to different ports of the Butler Matrix in order to obtain at the output a specifically designed phase distribution. The phase profiles achieved generate the interleaved beams (compared with the traditional scheme). Different DIM structures have been studied in order to find the best architecture in terms of symmetry, power balance and losses.

Figure 80 (left) shows the most straightforward structure that would be connected with the four input ports of the $4 \times 4$ Butler Matrix. As it is discussed in the following section, MZM Filters have been chosen to separate the carrier in one branch and the sideband in the other. In this configuration one of the components (carrier or sideband) is extracted from all the inputs of the DIM and guided to the first input. Thus, this component shows a reference phase distribution at the output. On the other side, the optical component that has not been redirected to the first input (sideband or carrier respectively) undertakes different phase distributions at the outputs of the Butler Matrix depending on the input chosen. 
Nevertheless, in order to obtain an optimal structure, the number of Y-junctions and crosses must be minimized. Y-junctions ideally induce $3 \mathrm{~dB}$ of power losses. Their number cannot be reduced but they can be, otherwise, spread in the matrix in order to reduce the overall amount of losses of the first input. On the other hand the crossings induce lower losses but the crosstalk with the transverse direction leads to phase errors in the phase distribution at the output of the Butler Matrix as it is explained below.

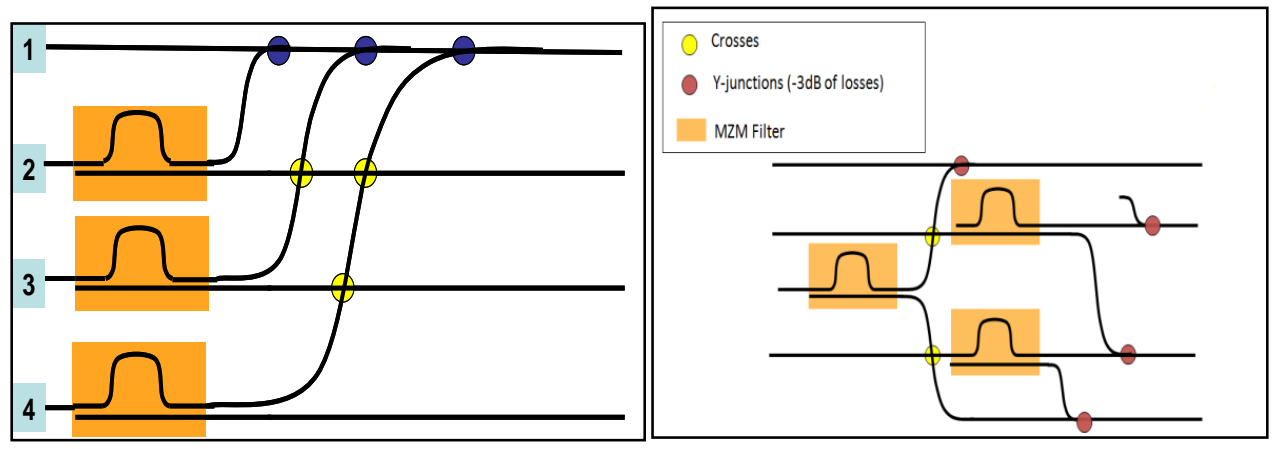

Figure 80. Initial DIM structure of $2 \mathrm{~N}$ archite cture (left) and alternative one (rigth) with better symmetry.

An alternative DIM structure is shown in Figure 80 (right). This design optimize s the symmetry and the losses are more equally distributed. This alternative shows the same performance than the structure showed in Figure 80 (left) but with different order in their ports. Additionally, the number of crossings is reduced and thus the undesired crosstalk signal at the input of the Butler Matrix is also reduced. Although the last alternatives improve the power balance and the losses, not all the branches have the same attenuations. For this reason, in the final design an additional Y-junction was added to equalize the losses and the output power in all the branches. This is the case of the Y-junction that has the second output of the DIM. 


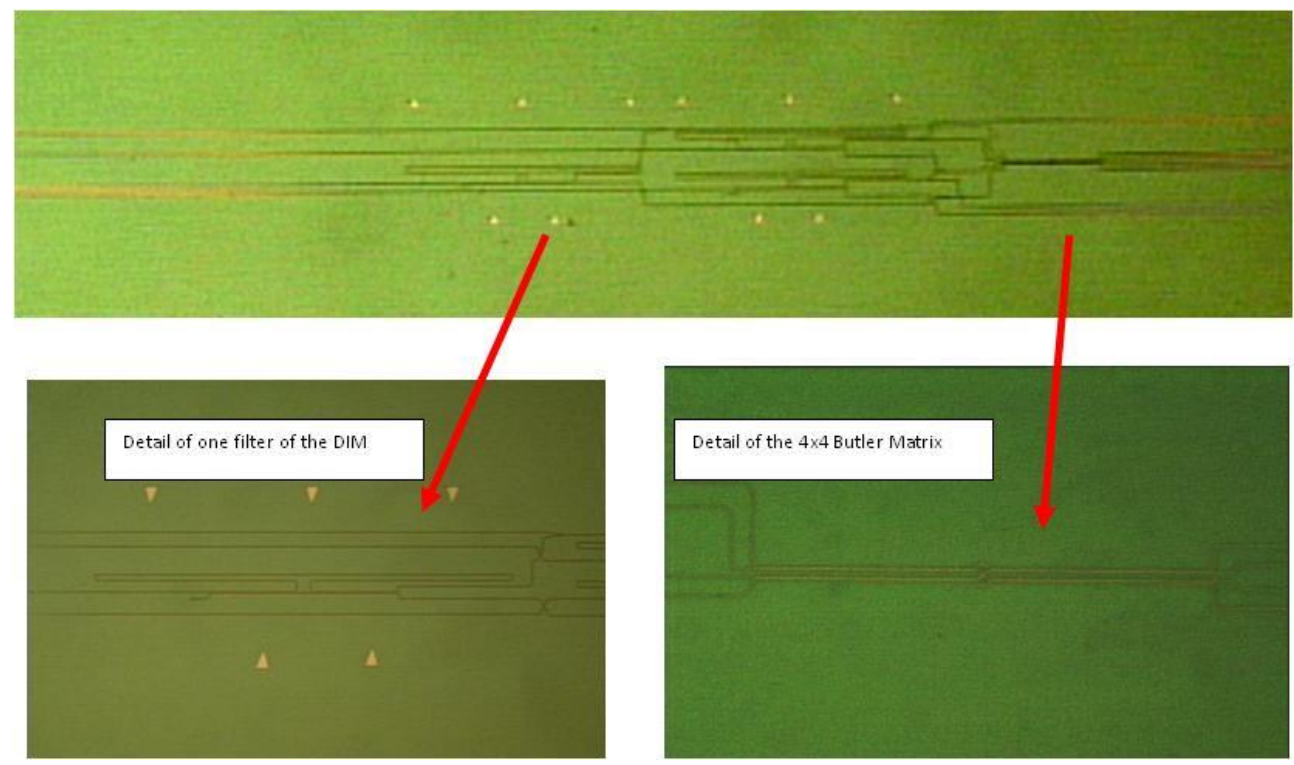

Figure 81. Optical micros cope image of a made DIM+Matrix sample.

Following the last structure, the samples that have been made anf analysed in the setup are composed by a DIM plus a $4 \times 4$ Butler matrix in order to obtain the expected phases at each of the four outputs. Figure 81 shows an optical microscope image of the made structure and a zoom in the detail of the matrix and the filter. In the following section, the implementation of the filters responsible of the extraction of one of the optical components is addressed.

\subsubsection{Results on Phase Distribution}

Figure 82 shows a schematic of the structure with the labelling of the input and output ports.

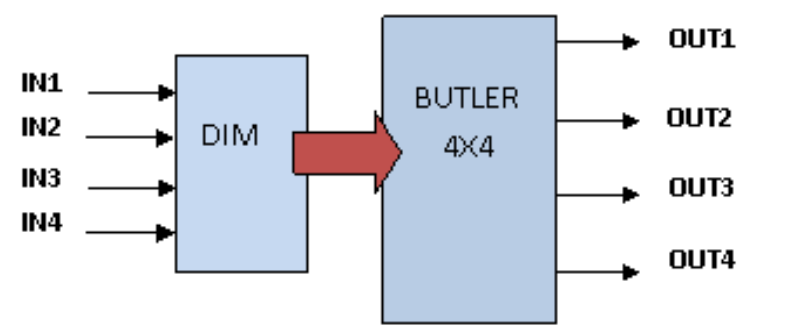

Figure 82. Structure of the DIM+Matrix chip. 
The average of the six measurements is shown in Table 17. The average of the phase has been calculated as the mean of the six relative phase tables and the error phase values have been calculated as the difference among relative an ideal phases.

\section{Table 17: Phase Performance of the DIM+Matrix s tructure (A VERAGE OF MEAS UREMENTS OF 6 CHIPS)}

\begin{tabular}{|c|c|c|c|c|c|c|c|c|c|}
\hline \multirow{6}{*}{$\begin{array}{l}\text { OUT1 } \\
\text { OUT2 } \\
\text { OUT3 } \\
\text { OUT4 }\end{array}$} & \multicolumn{4}{|c|}{ PHASE (ideal case) } & & & & & \\
\hline & \multirow{2}{*}{$\frac{\mid \mathrm{N} 1}{0}$} & \multirow{2}{*}{$\begin{array}{c}\text { IN2 } \\
0\end{array}$} & \multirow{2}{*}{$\begin{array}{c}\text { IN3 } \\
0\end{array}$} & \multirow[t]{2}{*}{ IN4 } & & \multicolumn{4}{|c|}{ AVERAGE OF SIX MEASUREMENTS } \\
\hline & & & & & & & & & \\
\hline & 0 & -180 & 180 & 0 & & \multicolumn{4}{|c|}{ PHASE (relative value) } \\
\hline & 0 & 270 & 90 & 180 & & IN1 & IN2 & IN3 & IN4 \\
\hline & 0 & 90 & 270 & 180 & OUT1 & $-1,12$ & $-17,67$ & 0,67 & $-3,49$ \\
\hline & & & & & OUT2 & $-2,00$ & $-154,92$ & 200,17 & $\begin{array}{l}-0,92 \\
-1005\end{array}$ \\
\hline & & & & & $\begin{array}{l}\text { OUT3 } \\
\text { OUT4 }\end{array}$ & $\begin{array}{l}2,78 \\
0,35\end{array}$ & $\begin{array}{c}262,98 \\
89,61\end{array}$ & $\begin{array}{c}84,88 \\
254,27\end{array}$ & $\begin{array}{l}180,95 \\
183,47\end{array}$ \\
\hline & & & & & OFFSET & 0,00 & 0,00 & 0,00 & 0,00 \\
\hline & & & & & & \multicolumn{3}{|c|}{ ERROR PHASE } & \\
\hline & & & & & & IN1 & IN2 & IN3 & IN4 \\
\hline & & & & & OUT1 & $-1,12$ & $-17,67$ & 0,67 & $-3,49$ \\
\hline & & & & & OUT2 & $-2,00$ & 25,08 & 20,17 & $-0,92$ \\
\hline & & & & & OUT3 & 2,78 & $-7,02$ & $-5,12$ & 0,95 \\
\hline & & & & & OUT4 & 0,35 & $-0,39$ & $-15,73$ & 3,47 \\
\hline & & & & & $\boldsymbol{\sigma}$ & 1,81 & 15,74 & 13,05 & 2,55 \\
\hline
\end{tabular}

It is seen from the average values that output phases related to inputs 2 and 3 show the worst results. Sigma values satisfy the specifications for inputs 1 and 4; however, inputs 2 and 3 are out of specifications. On the other hand, the overall error in these two inputs decreases due to the compensation of the errors in the measurements. 


\subsection{Conclusion}

In this chapter the design and characterization of a heterodyne optical Butler matrix have been reported. The more relevant performance parameters measured were the phase performance. From the results, the standard deviations in phase was 5.4 degrees considering all the port combinations.

The obtained results were validated from the system point of view. The degradation of the radiation diagram of the array antenna has been calculated from the performance calculations obtained for the $8 \times 8$ matrix.

In a second step, the heterodyne generation of a RF phase distribution with a photonic Butler Matrix was addressed. For that purpose, a 4x4 Butler Matrix was implemented together with a DIM. The DIM has been designed in order to separate the two optical components of the heterodyne optical signal and distribute them to the input ports of the Butler Matrix to obtain the desired phase distribution at the output of the beamformer structure. The design of the DIM structure was carried with the implementation of filtering structures that separate the optical components and waveguides, splitters, combiners and crossings to perform the distribution to the Butler Matrix.

From the results obtained, the feasibility of the heterodyne generation or RF phase distributions employing the photonic beamformer structure has been proven. The phase distribution specifications for the complete beamformer (two cascaded $8 \times 16)$ lead to a maximum deviation of 5 degrees.

The functionality of DIM is proven, and given the obtained results it cannot be discarded the viability of the DIM design implemented, but considering the not fully compliance of the results it is recommended to search for alternative options, such as an external generation of the optical components or alternative filtering structures can be explored. 


\section{CHAPTER 6}

\section{RF-like OBFN based on Integrated Optical Butler Matrices}

In this chapter, a different approach to optical Butler matrices is presented. Instead of using a DIM to separate the optical carrier and sideband, the Butler structure is used to provide propagation delays.

The photonic implementation of Butler matrices in guided-wave circuits was reported in chapter 5. As shown, the architectures were based on optical heterodyning, which requires very accurate control of the optical phase. A variant implementation following the technique proposed in [MAD02] have been demonstrated in chapter 5 in SOI PIC consisting in injecting the reference signal by an input port of the matrix which generates orthogonal beams interleaved with respect to the ones generated by the classical Butler matrix. In both cases, either generating the regular beams of the interleaved ones, the heterodyne generation technique described in the previous chapter is required which separates the optical spectrum in two components which are managed separately, inducing a phase shift in one of them, and afterwards combining both components again and reconstructing the original spectrum with a controlled phase difference between both components. Finally, this phase difference is translated to the photodetected RF signal.

The optical Butler matrix with a DIM for heterodyne mixing is able to perform $\mathrm{N}$ combinations of phase sets in the original configuration, and twice in the $2 \mathrm{~N}$ version. This may be interesting if one wants to increase the level at which adjacent 
beams overlap, that decreases the gain ripple over the whole antenna coverage, but degrades the isolation between adjacent beams.

Nevertheless, the mayor drawback of this heterodyne technique is that the required PIC path accuracy for phase-shifts multiple of 22.5 degrees at optical frequencies is extremely stringent in the range of a few nanometres as reported in chapter 5, which mandates to use manufacturing techniques with extremely high resolution, like SOI, and even in this case the minimum physical resolution is in the order of nanometres, the same order of the accuracy required. On the other hand, since each optical carrier has to be re-combined with the sideband at the output of the matrix, this puts stringent requirements on wavelength accuracy of the PIC device, requires precise control of the laser source wavelength and a thermal stabilization of the PIC to avoid drift of the filter responses.

The design proposed in this chapter try to overcome all the previous limitations of the heterodyne architectures by implementing the phase shift of the optical Butler matrix not in the optical domain but at the microwave frequencies by optical delays. In this case the advantages of miniaturization and manufacturing resolution of the photonic PIC technology are maintained. In this case, a phase shifter is implemented by a delay line calculated to correspond with the desired phase shift at the microwave frequency designed that is transported by the optical signal. This solution implemented as a PIC exhibits an accuracy in the phase implementation $10^{4}$ times better than in the heterodyne case.

The challenge is to build the basic building block of the matrix, a $2 \times 2$ 'RF-like hybrid coupler' with a unitary RF transfer matrix. As it is demonstrated by microwave circuit theory, only orthogonal sets of complex coefficients, when considering the two outputs from input 1 as the $1^{\text {st }}$ matrix row, and the two outputs from input 2 as the $2^{\text {nd }}$ matrix row, can avoid high losses through the coupler (losses meaning in fact that a strong part of the RF signal is reflected); besides it generates orthogonal beams in the sine space [MAI05].

Miniature pure optical couplers as the ones described in chapter 5 (coupled waveguides) can only provide 'in-RF phase' splitting (alias " 0 - $-R F$ " couplers). On the contrary, known hybrid couplers are from one of the two following types:

- " $90^{\circ}$ hybrids": phase-difference between outputs is $+90^{\circ}$ from one input, $-90^{\circ}$ from the other.

- " $180^{\circ}$ hybrids": phase-difference between outputs is $0^{\circ}$ from one input, $180^{\circ}$ from the other. 
In both cases a simple computation shows that the transfer matrix is "unitary" (i.e. the Hermitian product of two different columns is ' 0 ', and for any column multiplied by itself, it's ' 1 ').

\subsection{Design of the RF-Like Optical Butler}

\subsubsection{Basic Optical Coupler Equivalent to $2 \times 2$ Hybrid RF Coupler or 2x2 Butler Matrix}

The solution for the orthogonality is the implementation of an RF-like hybrid (the fundamental part of the Butler Matrix) by using $3 \mathrm{~dB}$ optical couplers and delay lines working as 90 degrees $(\lambda / 4)$ phase shifters $\left(e^{-j \pi / 2}=-j\right.$, if complex notation is used) at the design RF frequency. This concept is shown in the following figure:

$\ln 1$

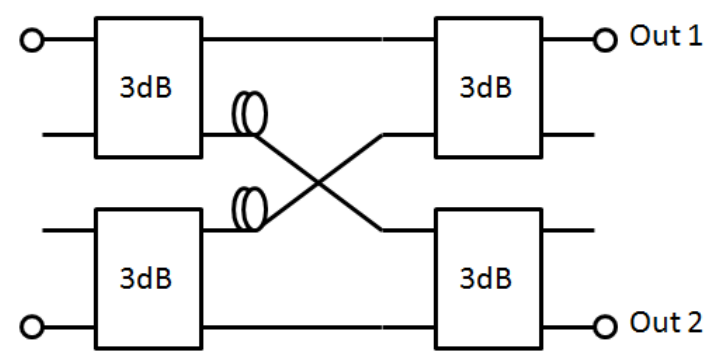

$$
\begin{gathered}
T=\frac{1}{\sqrt{2}}\left[\begin{array}{cc}
1 & -j \\
-j & 1
\end{array}\right] \\
T \times T^{*}=\left[\begin{array}{ll}
1 & 0 \\
0 & 1
\end{array}\right]=I
\end{gathered}
$$

Figure 83. RF-like 2x2 hybrid coupler scheme by us ing optical couplers and delay lines (left), the corresponding unitary RF transfer matrix $\mathbf{T}$ (right-up) and the demons tration of the orthogonality of T (right-down).

\subsubsection{4x4 Optical Butler Matrix}

The solution presented for $\mathrm{M}=2$, can be generalised to higher orders following the design rules of the RF Butler matrix as described in chapter 2. For the case of $M=4$, it is needed to introduce the classical basic delay for an $M^{\prime} \mathbf{x M}^{\prime}$ matrix between 2 stages of the basic hybrid couplers, and in-between them, which is $\lambda /(2 \mathrm{M})=\lambda / 8$, corresponding to a $\pi / 4=45$ degrees phase shift. Its transfer matrix $[\mathrm{T}]$ is actually unitary, and it provides regularly spaced phase-slopes at its outputs, as shown in Figure 84. 


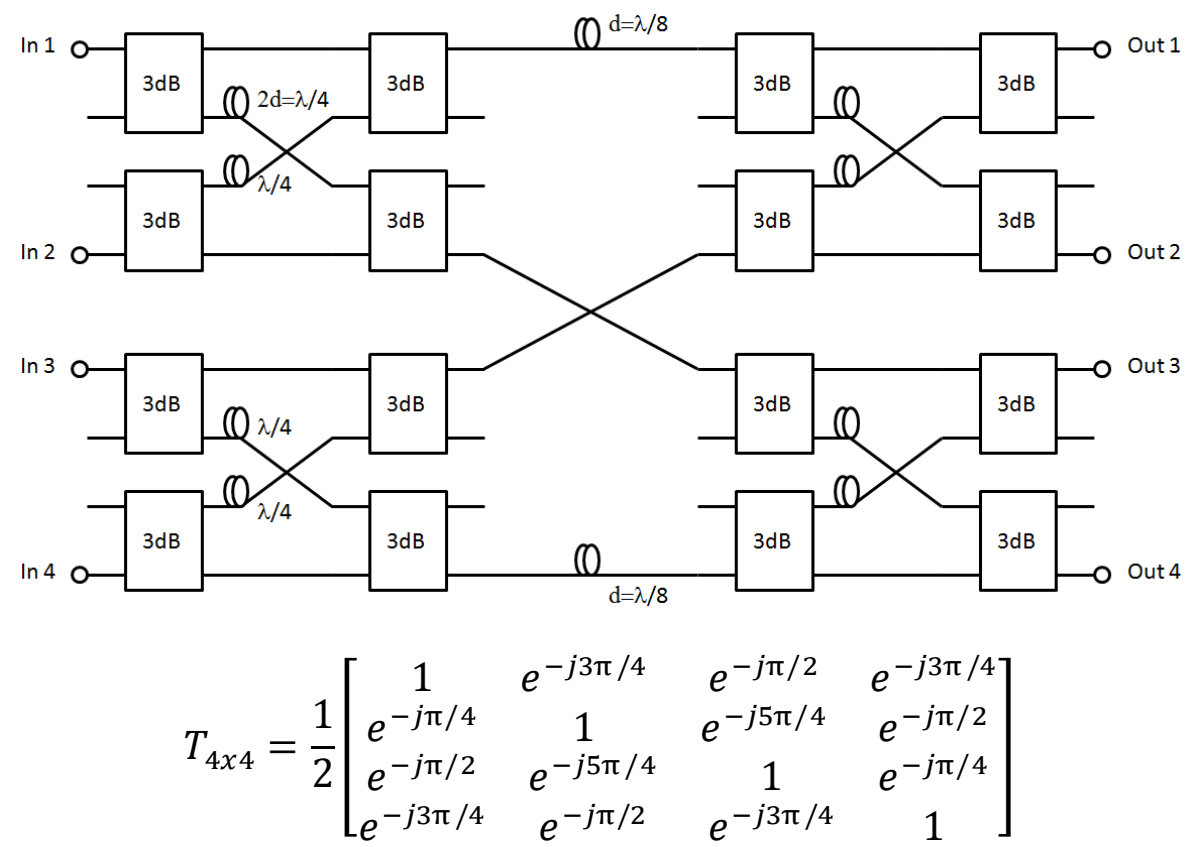

Figure 84. RF-like 4x4 B utler matrix (top) and its corresponding unitary RF transfer matrix $\mathrm{T} 4 \mathrm{x} 4$ (bottom).

In Figure $84, d$ is the unitary delay that implements the unitary phase shift within the matrix and depends on the number of ports $\mathrm{M}$, being $d=\lambda_{R F} / 2 M$. From a simple calculation it can be seen that $T_{4 x 4}$ is also orthogonal for the RF signal transported by the optical carrier.

A simplification can be implemented by combining intermediate stages by parallel optical paths reducing the number of $3 \mathrm{~dB}$ optical couplers. This presents the extra-advantage of avoiding the well-known $3 \mathrm{~dB}$ loss in the combiner, when incoming signals are not coherently in-phase (they will not be; in fact they are incoherent, as coming from different inputs; in other words, they are signals dedicated to different beams). 


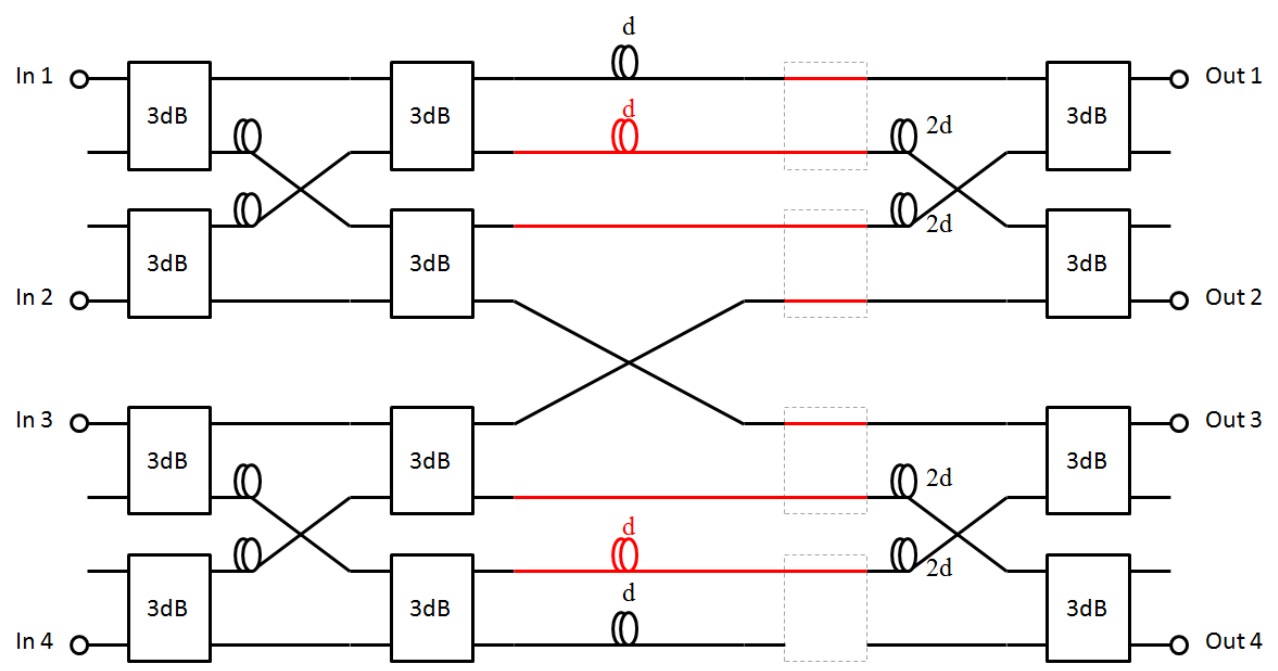

Figure 85. Simplifie d RF-like 4x4 B utler matrix with less 3 dB couplers.

\subsubsection{8x8 Optical Butler Matrix}

The simplified design of an RF-like 8x8 Butler Matrix is depicted in Figure 86. In the figure the squares indicate the structures working as a 90 degrees RF hybrid. In the bottom of the figure some calculations for the matrix parameters are included. The right side includes the calculated delay and corresponding phase of the output signal when entering in the port $4 \mathrm{~L}$ (in yellow), which corresponds with a differential phase of 157.5 degrees between adjacent ports. 


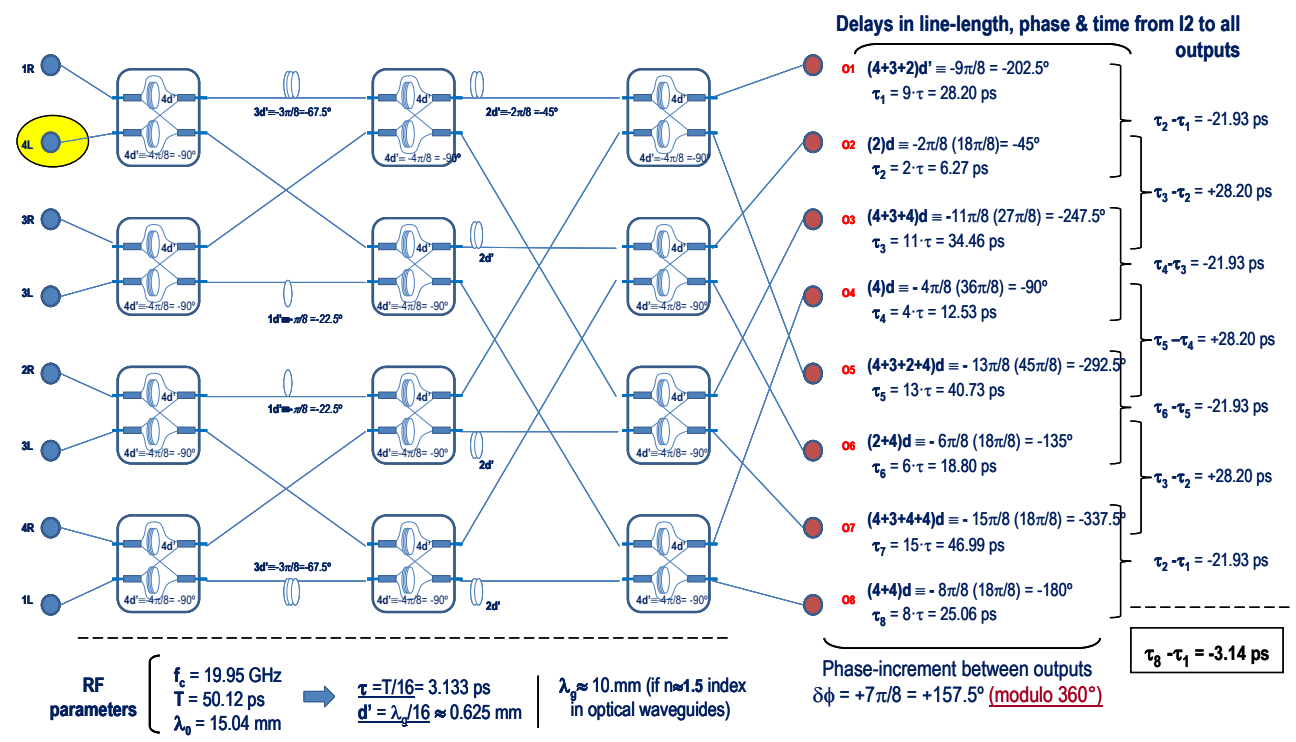

Figure 86 . Scheme of an optical Butler matrix of $8 \times 8$ ports imple mented by optical splitte rs/combiners and optical de lays.

It is possible to generalize the result in the same way as it is done in classical Butler matrices, i.e. adding new phase-delays multiples of $\pi / \mathrm{M} "=22.5$ degrees $[\mathrm{M} "=8]$, implemented in form of miniature optical waveguides with $\lambda_{\mathrm{RF}} / 2 \mathrm{M}$ " $=$ $\lambda_{\mathrm{RF}} / 16$ length (e.g. compact meander-lines). Again, we gather together each couple of successive " $3 \mathrm{~dB}$ combiner $+3 \mathrm{~dB}$ divider" by a "( 2 inputs) $\mathrm{x}$ ( 2 outputs) "in-RF phase' coupler". Only the $1^{\text {st }}$ stage of $3 \mathrm{~dB}$ dividers is kept, and the last stage of $3 \mathrm{~dB}$ combiners: these are '3-port' devices. All other are '4-port' devices, lossless if well designed, in other words, all the power from any input in only split into outputs and the sum of its parts at all these outputs is equal to the input power.

It is easy to check that the $8 \times 8$ Butler Matrix drawn in the previous figure, has a unitary matrix, so it can be lossless in principle, and in any case provide well decoupled beams, equally spaced if taking $\sin (\theta)$ as coordinate. The transfer matrix is the one presented in Figure 87 in which the columns (output vectors corresponding to eachinput) are orthogonal with respect to the Hermitian product in the $\mathrm{C}^{8}$ complex space. 
$\left[\frac{1}{2 \sqrt{2}}\left[\begin{array}{cccccccc}1 & e^{-j 7 \pi / 8} & e^{-j 3 \pi / 4} & e^{-j 9 \pi / 8} & e^{-j \pi / 2} & e^{-j 9 \pi / 8} & e^{-j 3 \pi / 4} & e^{-j 4 \pi / 8} \\ e^{-j \pi / 8} & 1 & e^{-j 11 \pi / 8} & e^{-j 3 \pi / 4} & e^{-j 7 \pi / 8} & e^{-j \pi / 2} & e^{-j 13 \pi / 8} & e^{-j 3 \pi / 4} \\ e^{-j \pi / 4} & e^{-j 9 \pi / 8} & 1 & e^{-j 3 \pi / 8} & e^{-j 5 \pi / 4} & e^{-j 15 \pi / 8} & e^{-j \pi / 2} & e^{-j 5 \pi / 8} \\ e^{-j 3 \pi / 8} & e^{-j \pi / 4} & e^{-j 5 \pi / 8} & 1 & e^{-j 13 \pi / 8} & e^{-j 5 \pi / 4} & e^{-j 11 \pi / 8} & e^{-j 2 \pi / 4} \\ e^{-j \pi / 2} & e^{-j 11 \pi / 8} & e^{-j 5 \pi / 4} & e^{-j 13 \pi / 8} & 1 & e^{-j 5 \pi / 8} & e^{-j \pi / 4} & e^{-j 3 \pi / 8} \\ e^{-j 5 \pi / 8} & e^{-j \pi / 2} & e^{-j 15 \pi / 8} & e^{-j 5 \pi / 4} & e^{-j 3 \pi / 8} & 1 & e^{-j 9 \pi / 8} & e^{-j \pi / 4} \\ e^{-j 3 \pi / 4} & e^{-j 13 \pi / 8} & e^{-j \pi / 2} & e^{-j 7 \pi / 8} & e^{-j 3 \pi / 4} & e^{-j 11 \pi / 8} & 1 & e^{-j \pi / 8} \\ e^{-j 7 \pi / 8} & e^{-j 3 \pi / 4} & e^{-j 9 \pi / 8} & e^{-j \pi / 2} & e^{-j 9 \pi / 8} & e^{-j 3 \pi / 4} & e^{-j 7 \pi / 8} & 1\end{array}\right]\right.$

\section{Figure 87. Transfer matrix [T] for the $\mathbf{8 x 8}$ matrix drawn in Figure $\mathbf{8 6}$.}

As described in chapter 5, a 3D beamforming network can be constructed by cascading sets of individual Butler Matrices, in this case of 8x8 port counts. For an optical beamformer feeding a DRA of $8 \times 8=64$ antenna elements with 64 independent, orthogonal beams, two sets of 8 matrices should be cascaded as is depicted in Figure 88. Electro-optical modulators and photodiodes should be placed at the inputs and output ports respectively. This configuration can be used in both transmission and reception modes with no changes, as occurs with the RF Butler matrices. The multibeam capability in transmission configuration is guaranteed by using wavelength division multiplexing, i.e., one wavelength for each beam. On the contrary, in reception the wavelengths should be associated to each antenna element.

The interconnection among the different parts of the beamformer can be done by optical fibre. The interconnection should be done with quite accuracy since difference in length in the connection between the two sets of Optical Butler Matrices $(\mathrm{OBM})$ and between the last OBM set and the photodiodes (in transmission configuration) will introduce unwanted delay and the corresponding phase error. Delay compensation can be done by individual miniature optical delay lines. As have been commented along the different chapters, it is interesting the use of the fiber optics to allocate the OBFM far from the antenna in order to reduce the complexity associated to the array. 


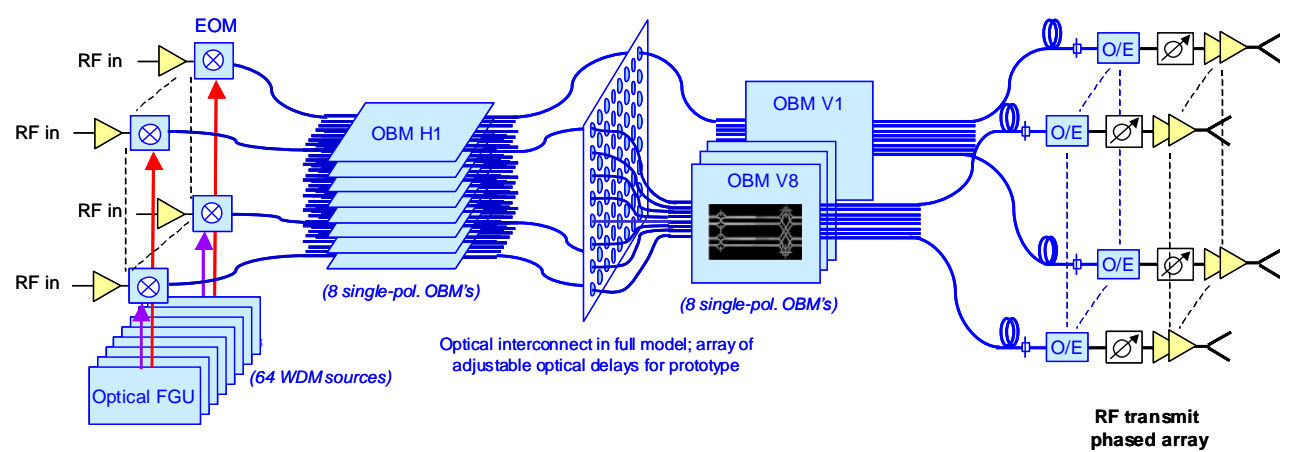

Figure 88. 64x64 Optical Beamforming Network bas ed on 8x8 RF-like OBMs. FGU: Frequency generation Unit; EOM: Electro-optical modulators

\subsection{RF-Like 8x8 Optical Butler PIC manufacturing}

The PIC has been manufactured in standard PLC (Germanium-Doped silica on silica) technology, which exhibits low insertion losses among the technologies available in the market for manufacturing lightwave integrated circuits. Low contrast silica-based optical waveguides consists of a germanium-doped silica $\left(\mathrm{SiO}_{2}-\mathrm{GeO}_{2}\right)$ layer on top of a silica $\left(\mathrm{SiO}_{2}\right)$ cladding layer deposited on a bare silicon substrate. The low contrast wafers used are made of a standard silicon wafer which is used as the substrate, $\mathrm{a} \mathrm{SiO}_{2}$ layer of a thickness of $15 \mu \mathrm{m}$ as the under and upper-claddings and a core layer of germanium-doped silica with a thickness of $6 \mu \mathrm{m}$ in order to realize the low refractive index contrast of $0.75 \%$ between the core and the claddings. Figure 89(a) shows a scanning electronic microscope (SEM) image of the waveguide which width has been chosen equal to $6 \mu \mathrm{m}$ to achieve single mode transmission for TE and TM polarization. Figure $89(\mathrm{~b})$ shows the mode profile at the operating wavelength of $1550 \mathrm{~nm}$. Propagation losses of $0.09 \mathrm{~dB} / \mathrm{cm}$ for straight waveguides and $0.2 \mathrm{~dB} / \mathrm{cm}$ for bended waveguides with bending radii of $5 \mathrm{~mm}$ have been measured with the manufacturing processes actually used.

The $3 \mathrm{~dB} 2 \times 2$ optical couplers have been designed as multimode interferometers (MMI). In order to select the optimum design for the MMI $3 \mathrm{~dB}$ splitters, different designs have been tested. In particular, the characterized MMIs structures had widths of $26,26.5$ and $27 \mu \mathrm{m}$ and lengths of 1594.5, 1595 and $1595.5 \mu \mathrm{m}$. Figure 90 shows a SEM picture of the MMI structures. 

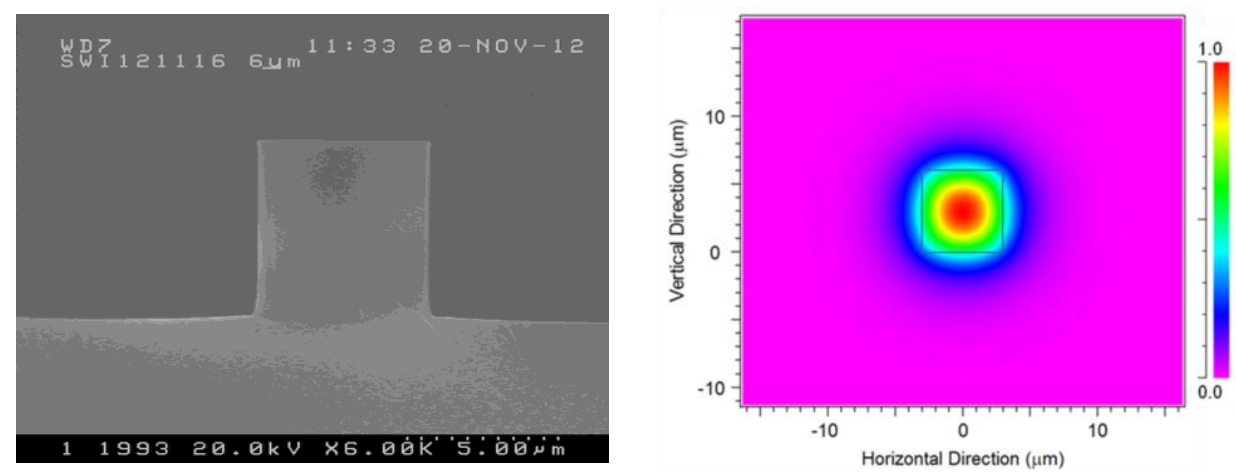

Figure 89. (a) SEM of a low contrast silica-based optical wave guide and (b) mode profile for a low contrast silica-based optical wave guide with a width and thickness of $6 \mu \mathrm{m}$. The extremely low roughness of the waveguide wall suggest that propagation losses lower than $0.09 \mathrm{~dB} / \mathrm{cm}$ can be achie ved once it will be covered with un-doped silica.
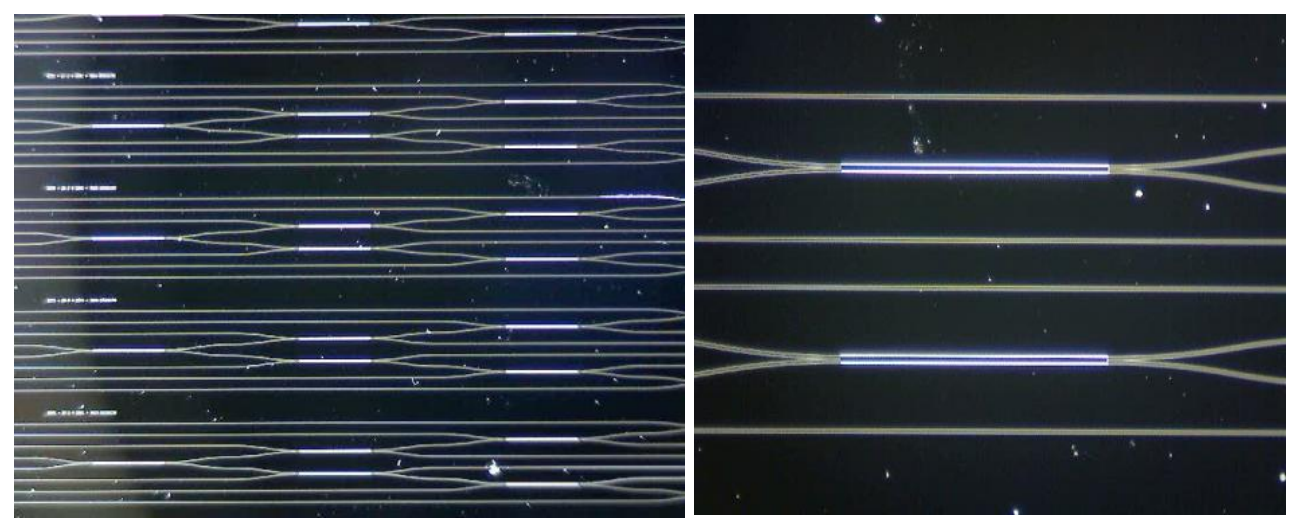

Figure 90. Microscope image of the MMI structures.

MMIs are very robust against fabrication tolerances regarding the splitting ratio and also have broadband operation. The following figure shows the wavelength dependency of the optical losses of a manufactured MMI. It can be seen as the response is flat in a wide range of wavelengths. 


\section{Port \#1}

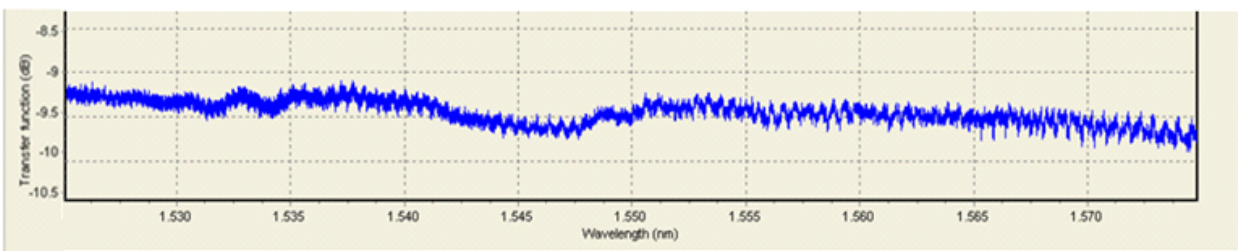

\section{Port \#2}

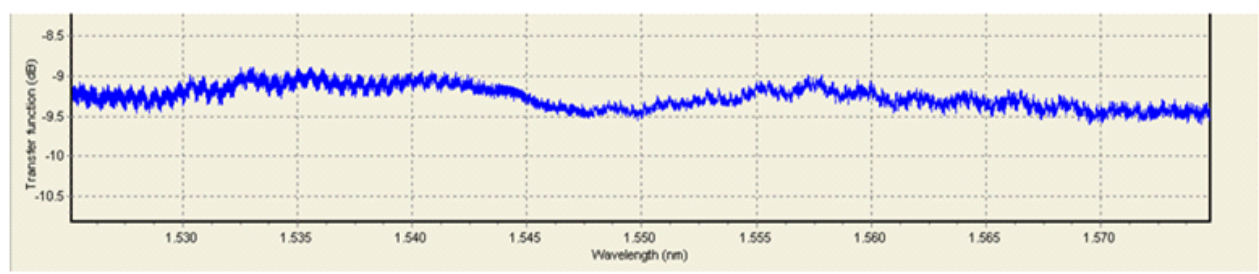

Figure 91. MMI response in a $50 \mathrm{~nm}$ wavele ngth. MMI with $26.5 \mu \mathrm{m}$ width and $1595 \mu \mathrm{m}$ length.

In the following figure the layout of the designed 8x8 RF-like Optical Butler Matrix is depicted. It can be appreciated that there is a number of optical crosses included in the design for power equalization (used as attenuators).

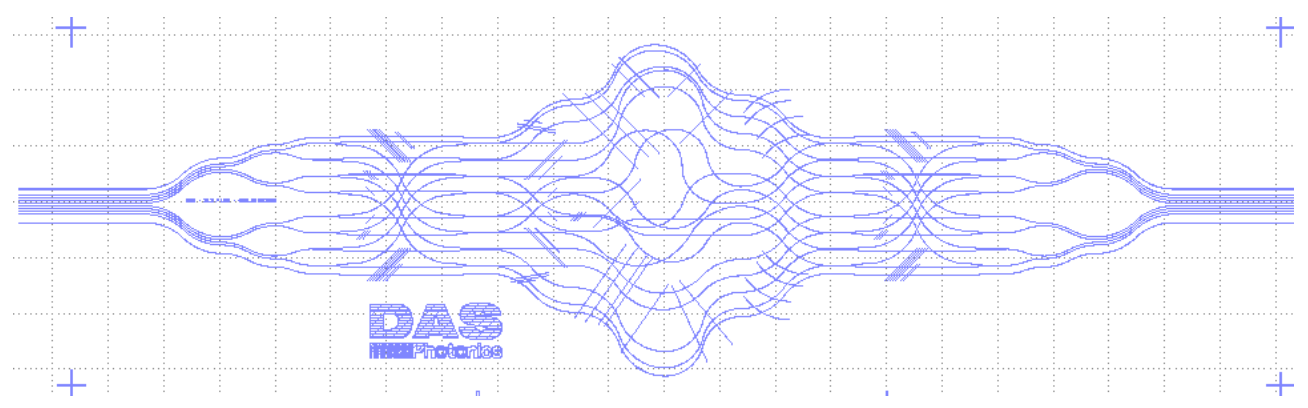

Figure 92. Layout of the designed 8x8 RF-like Optical Butler Matrix

The matrix was pigtailed with fibre arrays of 8 fibres and packaged in a metallic housing. The following figure shows the resulting component. 


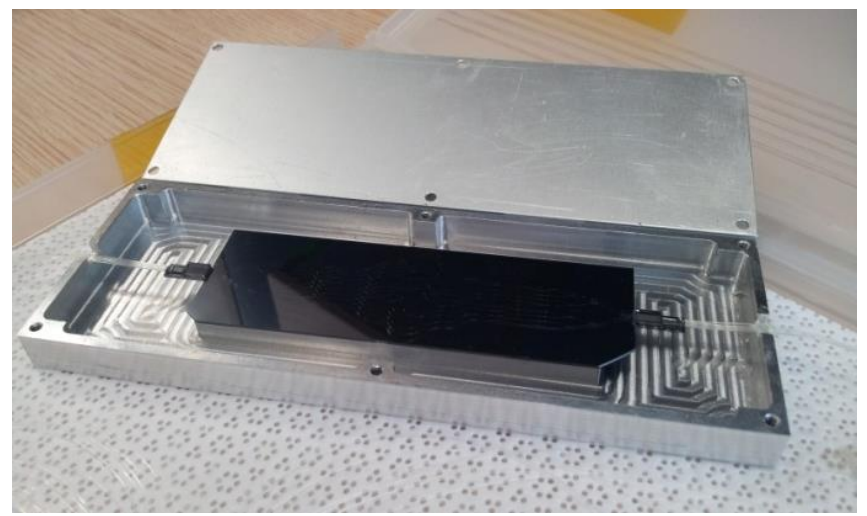

Figure 93. 8x8 RF-like Optical B utler Matrix Packaged and pigtailed.

\subsection{RF-Like 8x8 Optical Butler PIC Evaluation}

\subsubsection{Insertion Loss Performance}

The insertion loss of the matrix was measured for all the port combinations. The results are shown in the following table.

TABLE 18: OPTICAL INS ERTION LOSS OF THE PACKAGEID OBM CHIP.

\begin{tabular}{|c|c|c|c|c|c|c|c|c|c|c|c|}
\hline \multirow[b]{2}{*}{ Input Port } & \multicolumn{8}{|c|}{ Output Port } & \multirow[t]{2}{*}{$\begin{array}{l}\text { Stn Dev } \\
(\mathrm{dB})\end{array}$} & \multirow[t]{2}{*}{\begin{tabular}{|l} 
Mean loss \\
(dB)
\end{tabular}} & \multirow[t]{2}{*}{ IL (dB) } \\
\hline & 1 & 2 & 3 & \begin{tabular}{l|l}
4 \\
\end{tabular} & 5 & 6 & 7 & 8 & & & \\
\hline 1 & 20,5 & 19,8 & 20,2 & 19,6 & 20,1 & 19,5 & 19,2 & 19,5 & 0,43 & 19,8 & 10,8 \\
\hline 2 & 20,8 & 21,2 & 19,6 & 20 & 19,6 & 20,7 & 19 & 20 & 0,74 & 20,1 & 11,1 \\
\hline 3 & 19,5 & 20 & 19 & 19,6 & 19 & 20,1 & 19 & 20,5 & 0,57 & 19,6 & 10,6 \\
\hline 4 & 20,6 & 19,3 & 31,9 & 19,3 & 20,8 & 19,3 & 32,4 & 19,9 & 0,69 & 19,9 & 10,9 \\
\hline 5 & 20,6 & 19,3 & 20,8 & 19,8 & 20 & 18,6 & 19,7 & 19,4 & 0,71 & 19,8 & 10,8 \\
\hline 6 & 20,9 & 20,8 & 20,11 & 20,2 & 19,5 & 20,3 & 19,8 & 20,4 & 0,47 & 20,3 & 11,3 \\
\hline 7 & 19,5 & 20,3 & 19,8 & 20,7 & 19 & 20,4 & 19,9 & 21,6 & 0,79 & 20,2 & 11,2 \\
\hline \multirow[t]{2}{*}{8} & 20,9 & 19,2 & 32,54 & 20,2 & 21,1 & 19,6 & 31,5 & 20,9 & 0,78 & 20,3 & 11,3 \\
\hline & & & & & & & \multicolumn{2}{|c|}{ Mean $\sigma(\mathrm{dB})$ : } & 0,65 & & \\
\hline
\end{tabular}

Due to a problem in the manufacturing process, some bubbles appeared in one of the splitter structures of the PIC affecting the insertion losses of 4 measurements (shaded cells the table). The insertion losses of the matrix were around $10 \mathrm{~dB}$ (without considering the 1:8 splitting ratio) and the standard deviations of the losses among output ports was of $0.65 \mathrm{~dB}$. 


\subsubsection{Polarization and Wavelength Dependence}

As can be observed in the following figures, the power variation in a range of $55 \mathrm{~nm}$ is lower than $2.5 \mathrm{~dB}$ and the response is similar in both polarizations, so the OBM can be considered polarization independent and with broadband operation.

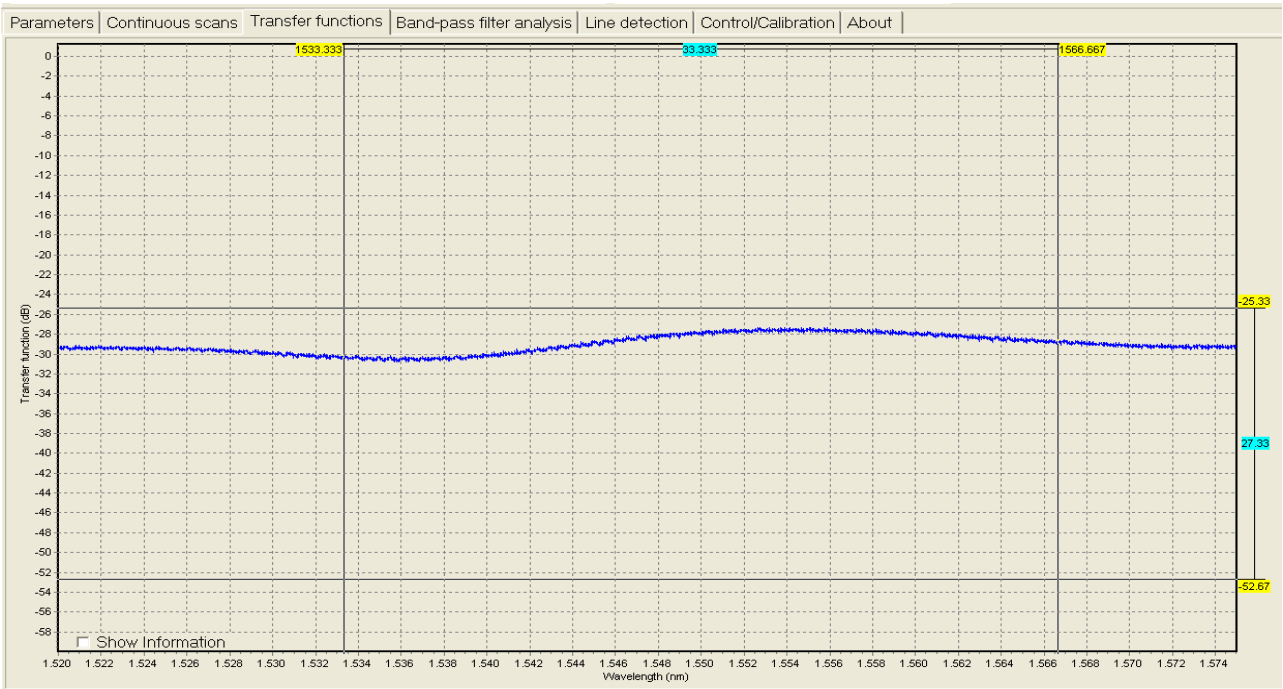

Figure 94. Optical Power vs wavelength for TE polarization.

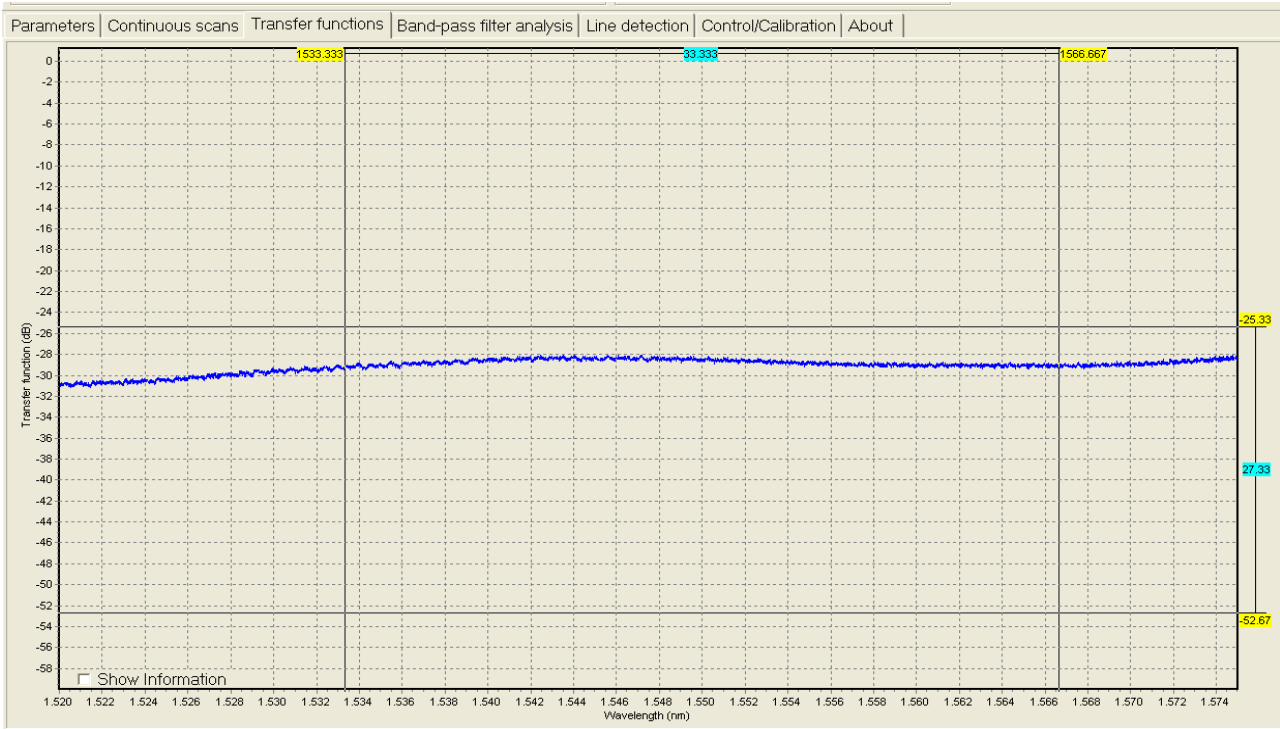

Figure 95. Optical Power vs wavelength for TM polarization. 


\subsubsection{Phase Shifting Performance of the Matrix}

Table 19 show the results after the measurements of the 64 relative phases of the $8 \mathrm{x} 8$ PIC measured at the central frequency of the band in which the matrix have been designed.

TABLE 19: Reilative PHAS ES TO THE OUT1 OF THE MATRIX @19.95GHz

\begin{tabular}{|c|c|c|c|c|c|c|c|c|c|c|}
\hline & & \multicolumn{8}{|c|}{ Relative phases to the OUT 1 of the PIC (@19.95GHz) } & \multirow{2}{*}{$\begin{array}{l}\text { Stand } \\
\text { Dev } \\
\text { (deg) }\end{array}$} \\
\hline & & OUT 1 & OUT2 & OUT3 & OUT4 & OUT5 & OUT6 & OUT7 & OUT8 & \\
\hline \multirow{2}{*}{ IN1 } & Theoretical & 0 & -157.5 & -45.0 & -112.5 & -90.0 & -67.5 & -135 & -22.5 & \multirow{2}{*}{0.12} \\
\hline & Measured & 0 & -157.6 & -44.8 & -112.4 & -90.0 & -67.7 & -134.9 & -22.5 & \\
\hline \multirow{2}{*}{ IN2 } & Theoretical & 0 & 157.5 & 45.0 & 112.5 & 90.0 & 67.5 & 135 & 22.5 & \multirow{2}{*}{2.73} \\
\hline & Measured & 0 & 164.0 & 47.6 & 116.9 & 91.5 & 68.1 & 139.4 & 20.7 & \\
\hline \multirow{2}{*}{ IN3 } & Theoretical & 0 & 67.5 & -135.0 & 202.5 & 90.0 & -22.5 & -45.0 & 112.5 & \multirow{2}{*}{2.31} \\
\hline & Measured & 0 & 71.9 & -136.4 & 206.9 & 91.3 & -24.1 & -44.6 & 112.9 & \\
\hline \multirow{2}{*}{ IN4 } & Theoretical & 0 & -67.5 & 135.0 & -202.5 & -90.0 & 22.5 & 45.0 & -112.5 & \multirow{2}{*}{1.93} \\
\hline & Measured & 0 & -64.6 & 139.0 & -202.6 & -90.8 & 23.1 & 43.4 & -113.2 & \\
\hline \multirow{2}{*}{ IN5 } & Theoretical & 0 & 22.5 & -45.0 & 67.5 & -90.0 & 112.5 & -135.0 & 157.5 & \multirow{2}{*}{1.49} \\
\hline & Measured & 0 & 27.6 & -42.4 & 70.4 & -88.5 & 115.2 & -133.7 & 160.3 & \\
\hline \multirow{2}{*}{ IN6 } & Theoretical & 0 & -22.5 & 45.0 & -67.5 & 90.0 & -112.5 & 135.0 & -157.5 & \multirow{2}{*}{2.71} \\
\hline & Measured & 0 & -19.0 & 49.6 & -65.5 & 93.3 & -113.2 & 138.6 & -160.8 & \\
\hline \multirow{2}{*}{ toN7 } & Theoretical & 0 & -112.5 & -135.0 & 22.5 & 90.0 & -202.5 & -45 & -67.5 & \multirow{2}{*}{2.00} \\
\hline & Measured & 0 & -109.9 & -133.0 & 23.4 & 91.6 & -205.8 & -43.4 & -69.0 & \\
\hline \multirow{2}{*}{ IN8 } & Theoretical & 0 & 112.5 & 135.0 & -22.5 & -90.0 & 202.5 & 45.0 & 67.5 & \multirow{2}{*}{2.47} \\
\hline & Measured & 0 & 118.7 & 136.7 & -21.5 & -89.4 & 206.2 & 43.1 & 68.1 & \\
\hline
\end{tabular}

The resulting standard deviation of the phases induced by the matrix is only 1.97 degrees respect to the theoretical ones. 


\subsection{Conclusion}

The innovative concept described in this chapter of an optical Butler matrix not requiring a heterodyne mixing for implementing the phase profiles have been described and a matrix of $8 \times 8$ ports designed to work at $19.95 \mathrm{GHz}\left(\mathrm{K}_{\mathrm{a}}\right.$ band in satellite communications) have been manufactured in low-contrast PIC technology. The promising performance anticipated in the introduction about the robustness of the phase and amplitude performance have been demonstrated obtaining a standard deviation of 1.97 degrees and $0.65 \mathrm{~dB}$ for the 64 possible combinations, which is a performance really good from the antenna point of view, especially the phase stability. Also the implementation of this structure in low-contrast PIC technology with square waveguides have exhibit a very broadband operation and a performance almost independent to the polarization, which simplifies very much its practical use. Finally, the promising of low size and mass comparing the RF technology have been also demonstrated. Due to the potential of this design, it has been protected by a patent in Europe, USA, Japan and Canada. 


\section{CHAPTER 7}

\section{Photonic \\ Microwave Signal \\ Generation}

\subsection{Introduction}

The last mile is at present the bottleneck that limits the data transfer rates provided to residential customers by telecom companies. This has led to the development of different broadband access networks both wired and wireless. Broadband wireless networks facilitate extending network coverage with relatively low deployment costs, faster revenue growth and increased flexibility compared to common cabled infrastructures.

In parallel, wired local area networks (LANs) and fibre-to-the-home (FTTH) access networks, which are experiencing a remarkable capacity increase, for instance $10 \mathrm{~Gb} / \mathrm{s}$ for Gigabit Ethernet (GbE, IEEE 802.3av), $10 \mathrm{~Gb} / \mathrm{s}$ for GPON (ITU G.987) are being widely deployed worldwide, as costs are decreasing due to higher market penetration, standardization, and use of low-cost optical technologies (e.g. [EFF10], [KIM15]).

This increasing capacity of wired data transmission has pushed the development of wireless technologies capable of transmitting high data-rate signals, which is only 
possible by utilizing the mm-wave band. In order to reduce the utilized bandwidth, efficient modulation techniques such as quadrature amplitude modulation (QAM) or phase-shifted keying (PSK) modulations are employed. Typically, the generation of such modulation formats at microwave or millimetre-wave frequencies is done electrically by generating the modulation at an intermediate frequency and up-converting this signal to the carrier frequency. However, when both the data-rate and the carrier frequency are high, the modulation has to be generated directly at the carrier frequency, which requires devices with very stringent constraints in terms of bandwidth and linearity.

\subsection{Photonic generation of wireless signals}

Radio-on-fibre (RoF) systems [COX04], [SEE06], [WAK10], [GHA11], [THO15] are signal distribution networks where optical fibre links are used to feed remote antenna units from a central location, being a particular case of the more general microwave photonic systems described in chapter 1 . In conventional (narrowband) wireless applications, the RF signals are generated, multiplexed and modulated in the base stations, i.e. close the antenna. Optical fibre allows the centralization of these functions since it can distribute signals with very low attenuation as described in chapter 1 . Thus, the base stations can be significantly simplified to just provide the optoelectronic conversion, power amplification and radiation. Additionally, a centralized architecture allows cost savings by sharing equipment between a set of base stations and easing operation and maintenance tasks and simplifies the dynamic allocation of resources (such as frequency channels or time slots). These benefits are particularly relevant in mobile networks providing high bit rates since these reduce the coverage area of the base stations and therefore make extensive use of micro- and pico-cells.

RoF has been proposed as an enabling technology for the transparent distribution of radiofrequency signals. The characteristics of the photonic technology such as huge electrical bandwidth, low weight, volume, consumption, low attenuation or potential high-level integration do this technology very suitable for these applications. Thus, for example, RoF can be used to feed mobile base stations (fiber backhaul) increasing the aggregated traffic that can be served by the base station in comparison to the conventional approach of using microwave radiolinks. Using this approach, any present or future mobile standard can be distributed to the base stations. On the other hand, RoF systems have been shown able to overcome the limitations of current electrical systems demonstrating multi gigabit-per-second data rates wireless transmission at the mm-wave band [HIR03], [OHA03], [NAG04], [PIQ06a]. 
However, photonics, as described in chapter 1, can go beyond the distribution of wireless signals. Optical components can be used to combine optical distribution with optical generation of microwave and millimetre-wave signals. Traditional schemes for the generation of high-frequency electrical signals are based on generating the data signal at baseband and frequency up-converting it by using techniques of harmonic mixing. Thus, these techniques use nonlinear mechanisms to move the signals to higher frequencies and need proper filtering to eliminate harmonics or spurious signals. Besides, if the frequency is high enough, several stages of up-conversion and filtering are needed. These processes degrade the signal quality, impose huge bandwidth requirements to the RF front-end and limit considerably the usable bandwidth besides of not providing too much flexibility to dynamically change the frequency of operation, the modulation format or the system bandwidth. Thus, in channels with bitrates in the range of Gbps at frequencies of 40 $\mathrm{GHz}$ to $100 \mathrm{GHz}$ and beyond, the traditional electrical schemes are inadequate, especially if modulations of high spectral efficiency such as M-QAM or OFDM where linearity is critical are employed.

Up-conversion can alternatively be done directly in the optical domain [GOP93], [COR01], [THO15a]. Optical upconversion can be achieved by using the nonlinearity of the external modulator (typically a MZM), by employing wavelength conversion techniques [JIA05] or by exploiting the photo-detector's non-linearity [CHO05]. Most published schemes are based on the family of MZM-assisted optical upconversion techniques due to its simplicity and efficiency. Another advantage of MZM-assisted optical upconversion is the ability to parallelize the mechanism to generate a set of signals simultaneously.

A step further is to combine distribution, up-conversion with the building of the multilevel signal by using fiber optics, what is known as microwave photonic vector modulation. There are also pure-microwave schemes to generate the multilevel signal directly at the carrier frequency and thus avoid the need of harmonic mixing and filtering. In this way, electrical vector modulators have been developed in MMIC technology up to $110 \mathrm{GHz}$ [MCP01], where a baseband (60 Mbps) signal is processed and injected into the modulator. It can add additional functionalities as linearization, filtering or pre-distortion by exploiting the baseband processing [CHO00]. However, electrical vector modulation has the limitation of bandwidth due to the technology employed in its design. This limits the potential of this approach to reach Gbps rates. At this point is where optical technology stands out since with this technology there are no fundamental limits in terms of bandwidth.

An optical implementation of a vector modulator allows the exploitation of the benefits of fiber optical components to deal with broadband microwave signals plus the easiness in distribution. In this sense, several approaches have been proposed to 
realize quadrature modulation [JEM02], [CHA02], [CAN03], [HIR03], [LOA04], [YIF05], [SEE06], [SAM07], [SAM08], [COR08], [ZHA08], [JIA10]. Typically, they are based on the vector summation technique, which consists in achieving the desired carrier phase and amplitude by summing two orthogonal components (named in-phase and quadrature components). Thus, in the proposed techniques two or more broadband electro-optical modulators are needed, as well as complicated electrical control signals and electrical hybrids at the carrier frequency, which limits the useful bandwidth and increases the hardware complexity.

\subsection{Fundamentals of Quadrature Modulations}

Quadrature Amplitude Modulation or QAM is a form of modulation which is widely used for modulating data signals onto a carrier used for radio communications. It is widely used because it offers advantages over other forms of data modulation such as PSK, although many forms of data modulation operate alongside each other.

In digital communications, modulation is often expressed in terms of I (in-phase) and Q (quadrature) as shown in Figure 96. This is a rectangular representation of the polar diagram. On a polar diagram, the I axis lies on the zero degree phase reference, and the Q axis is rotated by 90 degrees. The signal vector's projection onto the I axis is its " $\mathrm{I}$ " component and the projection onto the Q axis is its "Q" component.

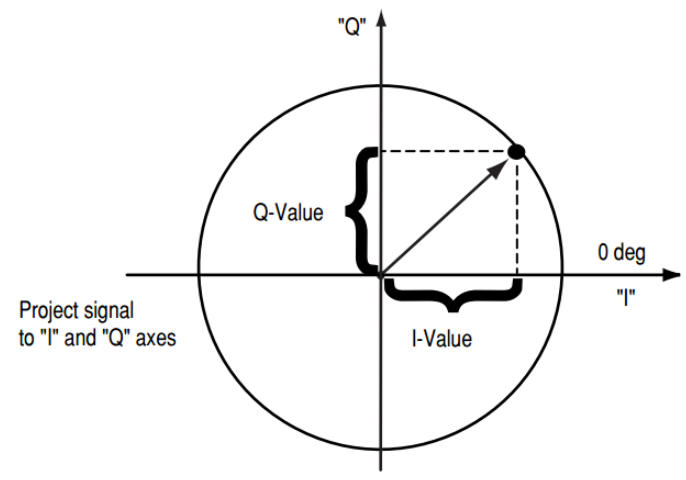

Polar to Rectangular Conversion

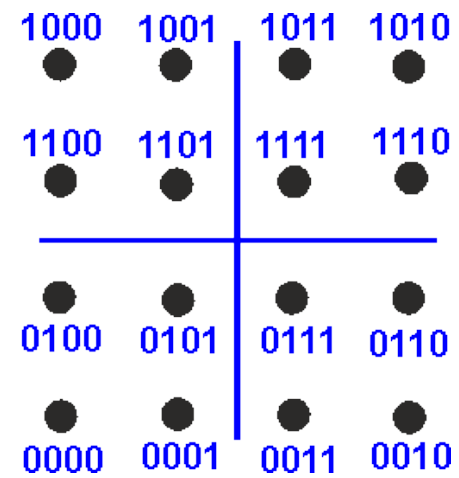

Figure 96. Rectangular representation of the polar diagram (left) and B it sequence mapping for a $16 \mathrm{QAM}$ signal.

Quadrature Amplitude Modulation is a signal in which two carriers shifted in phase by 90 degrees are modulated and the resultant output consists of both amplitude and phase variations. As both amplitude and phase variations are present 
it may also be considered as a mixture of amplitude and phase modulation. When used for digital transmission for radio communications applications is able to carry higher data rates than ordinary amplitude modulated schemes and phase modulated schemes. As with phase shift keying, the number of points at which the signal can rest, i.e. the number of points on the constellation is indicated in the modulation format description, e.g. 16QAM uses a 16 points constellation. When using QAM, the constellation points are normally arranged in a square grid with equal vertical and horizontal spacing and as a result the most common forms of QAM use a constellation with the number of points equal to a power of 2 i.e. 16, 64, 256 and they are referred to as 16QAM, 64QAM and 256QAM.

The advantage of moving to the higher order formats is that there are more points within the constellation and therefore it is possible to transmit more bits per symbol. The downside is that the constellation points are closer together and therefore the link is more susceptible to noise. As a result, higher order versions of QAM are only used when there is a sufficiently high signal to noise ratio. To provide an example of how QAM operates, the constellation diagram in Figure 96 shows the values associated with the different states for a 16QAM signal. From this it can be seen that a continuous bit stream may be grouped in groups of four bits and represented as a sequence of symbols. Normally the lowest order QAM encountered is 16QAM. The reason for this being the lowest order normally encountered is that 2QAM is the same as binary phase-shift keying, BPSK, and 4QAM is the same as quadrature phase-shift keying, QPSK.

\subsubsection{QAM Theory and Formulas}

The basic QAM theory aims to express the operation of QAM, quadrature amplitude modulation using some mathematical formulae. Fortunately, it is possible to express some of the basic QAM theory in terms of relatively simple equations that provide some insight into what is actually happening within the QAM signal.

Quadrature amplitude theory states that both amplitude and phase change within a QAM signal. The basic way in which a QAM signal can be generated is to generate two signals that are 90 degrees out of phase with each other and then sum them. This will generate a signal that is the sum of both waves, which has a certain amplitude resulting from the sum of both signals and a phase which again is dependent upon the sum of the signals. If the amplitude of one of the signals is adjusted then this affects both the phase and amplitude of the overall signal, the phase tending towards that of the signal with the higher amplitude content.

As there are two RF signals that can be modulated, these are referred to as the I - In-phase and Q - Quadrature signals. The I and Q signals can be represented by the equations below: 


$$
I=A \cos (\psi) \text { and } Q=A \sin (\psi)
$$

It can be seen that the I and Q components are represented as cosine and sine. This is because the two signals are 90 degrees out of phase with one another. Using the expression $\mathrm{A} \cos (2 \pi \mathrm{ft}+\Psi)$ for the QAM modulated carrier signal, it can be decomposed in its I and $\mathrm{Q}$ components as:

$$
A \cos (2 \pi f t+\psi)=I \cos (2 \pi f t)-Q \sin (2 \pi f t)
$$

where $f$ is the carrier frequency. This expression shows the resulting waveform is a periodic signal for which the phase can be adjusted by changing the amplitude of either or both I and Q. This can also result in an amplitude change as well. Accordingly, it is possible to digitally modulate a carrier signal by adjusting the amplitude of the two mixed signals.

The advantage of using QAM is that it is a higher order form of modulation and as a result it is able to carry more bits of information per symbol. By selecting a higher order format of QAM, the data rate of a link can be increased.

\subsubsection{QAM Modulator \& Demodulator}

The QAM modulator and QAM demodulator are key elements within any quadrature amplitude modulation system. The modulator and demodulator are used to encode the signal, often data, onto the radio frequency carrier that is to be transmitted. Then the demodulator is used at the remote end to extract the signal from the RF carrier so that it can used at the remote end.

The QAM modulator essentially follows the idea that can be seen from the basic QAM theory where there are two carrier signals with a phase shift of 90 degrees between them. Then, these are amplitude modulated with the two data streams known as the I or In-phase and the Q or quadrature data streams. These are generated in the baseband processing area. The two resultant signals are summed and then processed as required in the RF signal chain, typically converting them in frequency to the required final frequency and amplifying them as required. 

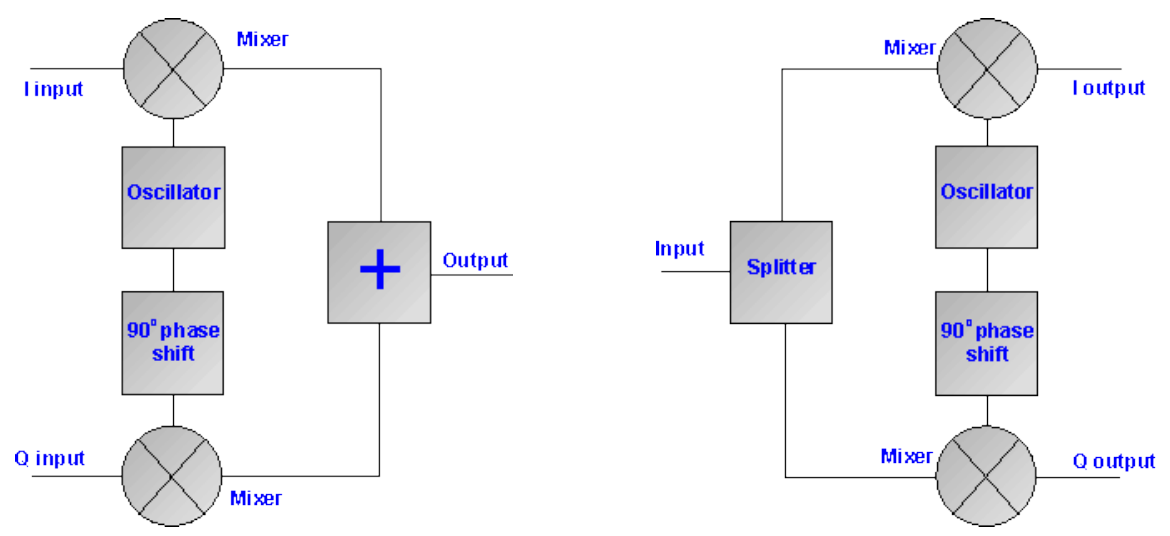

Figure 97. Basic QAM modulator (left) and de modulator (right) diagram.

The QAM demodulator is very much the reverse of the QAM modulator. The signals enter the system, they are split and each side is applied to a mixer. One half has the in-phase local oscillator applied and the other half has the quadrature oscillator signal applied.

The basic modulator assumes that the two quadrature signals remain exactly in quadrature. A further requirement is to derive a local oscillator signal for the demodulation that is exactly on the required frequency for the signal. Any frequency offset will be a change in the phase of the local oscillator signal with respect to the two double sideband suppressed carrier constituents of the overall signal. Systems include circuitry for carrier recovery that often utilizes a phase locked loop. Recovering the phase of the carrier is an important stage, otherwise the bit error rate for the data will be compromised. 


\title{
CHAPTER 8
}

\author{
Photonic \\ Generation of Electrical \\ Vector Modulations at \\ Microwave/Millimetre-wave \\ Frequencies
}

\subsection{Photonic Vector QPSK Modulator Architecture}

A schematic of the proposed PVM architecture is depicted in Figure 98.

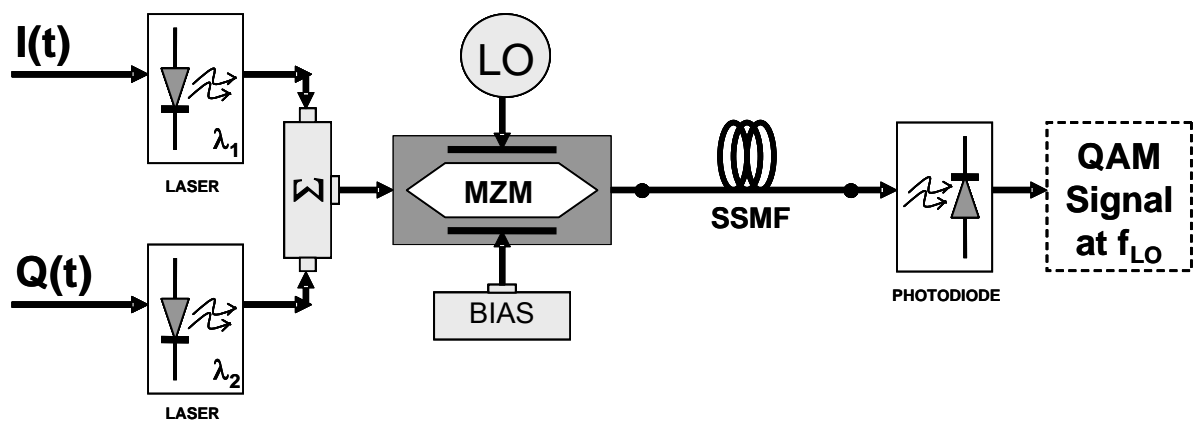

Figure 98. Photonic vector modulator (PVM) architecture. 
Two semiconductor lasers, emitting at wavelengths $\lambda_{1}$ and $\lambda_{2}$ and linearly biased are directly modulated by two baseband signals corresponding to the modulating $\mathrm{I}(\mathrm{t})$ and $\mathrm{Q}(\mathrm{t})$ driving currents. Both optically modulated signals are combined and externally modulated by a microwave/mm-wave local oscillator (LO) frequency tone in an external quadrature-biased Mach-Zehnder modulator (MZM). Since MZMs are optical-polarization dependent devices, polarization controllers or polarizationmaintaining fibre must be employed. Then, the signals are transmitted over a standard single-mode fibre (SSMF) optical link, whose length and dispersion are $L$ (m) and $D(\mathrm{ps} / \mathrm{km} \cdot \mathrm{nm})$ respectively, and photodetected. The fibre's chromatic dispersion induces a delay between both I- and Q-modulated optical signals equal to $\Delta \tau=D \cdot L \cdot \Delta \lambda$, where $\Delta \lambda=\lambda_{2}-\lambda_{1}$ is the optical wavelength spacing. This delay can be tuned by changing the fibre length or the wavelength spacing in order to obtain a delay of a quarter of the microwave LO tone period (named quadrature condition), which corresponds with a differential phase of $\pi / 2$ radians between the I and Q LO components. The RF component of the photocurrent for quadrature condition is given by [COR01]:

$$
\begin{aligned}
i_{P D}^{(R F)}(t) & =\left(\frac{\Re t_{f f} P \sqrt{2}}{L_{\Sigma} L_{F I B}}\right) J_{0}\left(\frac{m_{L O}}{2}\right) J_{1}\left(\frac{m_{L O}}{2}\right) \sin \left(\omega_{L O} t+3 \pi / 4\right) \\
& +\left(\frac{\mathfrak{R} t_{f f} \eta}{L_{\Sigma} L_{F I B}}\right) J_{0}\left(\frac{m_{L O}}{2}\right) J_{1}\left(\frac{m_{L O}}{2}\right) \\
& \left\{I(t) \sin \left(\omega_{L O} t-\pi / 2\right)+Q(t) \sin \left(\omega_{L O} t\right)\right\}
\end{aligned}
$$

where $I(t)$ and $Q(t)$ are the modulating currents driving the semiconductor lasers, both varying in the $\pm \Delta I \mathrm{~A}$ margin, $\omega_{\mathrm{LO}}$ is the LO angular frequency, $R$ is the photodiode responsivity, $t_{f f}$ is the MZM insertion loss, $P=P_{\text {LASER }}\left(I_{B I A S}\right)$ is the mean optical power emitted by the optical sources (biased at the centre of the linear zone of the power-vs-current transfer function $\left.P_{L A S E R}(i)\right), L_{\Sigma}$ and $L_{F I B}$ are the losses of optical combiner and optical link respectively, $\eta$ is the slope efficiency of the laser diodes in W/A, $J_{0}$ and $J_{I}$ are the zero-th and first order Bessel functions of the first kind, respectively, and $m_{L O}=\pi V_{L O} / V_{\pi}, V_{L O}$ being the amplitude of the local oscillator tone and $V_{\pi}$ being the MZM half-wave voltage.

From (8.1) it can be observed that data signals are quadrature modulated over the LO carrier since a $\pi / 2$ radians phase difference induced in the sine argument by the fibre's chromatic dispersion [COR01]. Moreover, an unmodulated LO tone remains in the signal. The power ratio between this undesired tone and the modulated signal is $(P / \Delta I \eta)^{2}$. For optimum laser modulation - in which the lineal margin of 
the power-vs-current transfer function is completely covered $-P \approx \Delta I \eta$ is satisfied being the LO-tone and the modulated-signal power similar. In this case, the overall signal power is $3 d B$ greater than the modulated-signal one. The LO tone only stands for a DC level when the quadrature signal is received and demodulated. It can also generate a low-frequency tone in the case where frequency detuning occurs during the demodulation process. In both cases, it can be easily removed by high pass filtering the received in-phase $(I(t))$ and quadrature-phase $(Q(t))$ base-band signal components without quality degradation. A detailed analysis of the mathematical model of the architecture is described in Annex C.

It is worth pointing out that no electrical devices that introduce complexity and impose bandwidth limitations — such as hybrids couplers, combiners, or filters are needed in the generation process. Moreover, the optical sources are modulated by baseband electrical signals and, therefore, low cost, low threshold current and high in-chip integration optical sources, such as VCSELs [KIM99], can be employed. Besides, high power broadband baseband signals can be easily generated by commercial low cost digital-to-analogue technology. Finally, only one optical modulator is needed in the PVM architecture, representing a significant complexity reduction when compared to previously proposed approaches [JEM02], [CAN03], [LOA04].

\subsubsection{Performance Analysis}

The PVM main system parameters must be adjusted for a given local oscillator frequency. This adjustment is realized by properly tuning the optical fibre length or the wavelength spacing between optical wavelengths for a given dispersion parameter in order to keep the $D \cdot L \cdot \Delta \lambda$ product (delay induced by the chromatic dispersion) constant. Therefore, tuning errors will produce a system performance penalty - in particular, a deviation in the relative received LO phases that moves them away from the quadrature state. Considering a maximum received LO phase deviation of 1 degree as a performance limit, the tolerances in the fibre length and wavelength spacing for different $\mathrm{LO}$ frequencies are shown in Table 20 assuming a fibre dispersion parameter of $D=17 \mathrm{ps} / \mathrm{km} \cdot \mathrm{nm}$, which is typical for SSMF around $1550 \mathrm{~nm}$. As can be seen in Table 20, there is a reasonable tolerance in the tuning parameters (e.g. 1.3 meter in fibre length for a $40 \mathrm{GHz} \mathrm{LO}$ frequency) so it can be concluded that the proposed architecture can be readily adjusted using commercial length measurement tools such as an optical time domain reflectometer, whose precision is sufficient for the considered tolerance. In addition, the useful bandwidth for the LO frequency, considering the 1 degree error criterion, is shown in Table 20 for four different $\mathrm{LO}$ frequencies. 


\section{TABLE 20: SYS TEM TOLERANCES AND LO TUNING BANDWIDTH FOR DIFFERENT LO FREQUENCIES CONS IDERING A MAXIMUM QUADRATURE PHASE ERROR OF 1 DEGREE. FIBRE DIS PERS ION PARAMETER FOR DEPICTED VALUES IS $D=17 \mathrm{PS} / \mathrm{KM} \cdot \mathrm{NM}$}

\begin{tabular}{|c|c|c|c|c|}
\hline LO frequency $(\mathrm{GHz})$ & 5 & 10 & 40 & 60 \\
\hline \hline Fiber length tolerance $(\mathrm{m})$ & 10.2 & 5.1 & 1.3 & 0.9 \\
\hline Maximum wavelength spacing error $(\mathrm{pm})$ & \multicolumn{5}{|c|}{8.9} \\
Nominal wavelength spacing of $800 \mathrm{pm}$ & \multicolumn{5}{|c|}{35.6} \\
Nominal wavelength spacing of $3200 \mathrm{pm}$ & \multicolumn{5}{|c|}{} \\
\hline LO tuning bandwidth $(\mathrm{MHz})$ & 110 & 220 & 890 & 1330 \\
\hline
\end{tabular}

By replacing one of the two lasers with a tuneable laser, the proposed architecture can be upgraded to enable its use in systems with multiple remote base stations with dynamic signal distribution and delivery. So, the fibre link connecting the MZM and the PD in the original scheme can be employed as a fibre span connecting the generation station and remote base stations. With the proposed upgrade, the wavelength spacing between I and Q optical signals can be dynamically tuned to maintain the quadrature condition when the fibre link distance changes due to the reallocation of the fed remote base station.

\subsubsection{Experimental Results}

Due to the lack of directly-modulated lasers in the laboratory, two externally modulated continuous wave DFB optical sources spaced $0.8 \mathrm{~nm}$ apart were used in the experimental setup to verify the performance of the proposed system. Two MZMs $\left(\mathrm{V}_{\pi}=5 \mathrm{~V}\right)$ were employed to modulate the $\mathrm{I}$ and $\mathrm{Q}$ baseband signal components, which were obtained from a digital transmitter configured to generate a QPSK modulation with a raised root cosine filtering and a 0.35 roll-off factor. The I- and Q-modulated optical signals were combined by means of a WDM multiplexer and polarization controlled, amplified in an Erbium-doped fibre amplifier and again externally modulated by a $37.6-\mathrm{GHz}$ LO tone using a third $\mathrm{MZM}\left(\mathrm{V}_{\pi}=5 \mathrm{~V}\right)$. The modulated optical signal was transmitted over a SSMF fibre span of 340 meters length with a dispersion parameter of $D=16.34 \mathrm{ps} /(\mathrm{km} \cdot \mathrm{nm})$ and received in a photodetector (PD) with $\mathfrak{R}=0.7 \mathrm{~A} / \mathrm{W}$ responsivity and a $50 \mathrm{GHz}$ bandwidth. The optical spectrum at the photodiode input is depicted in Figure 99, in which the two optical carriers corresponding to the I and Q components, respectively, can be observed. 


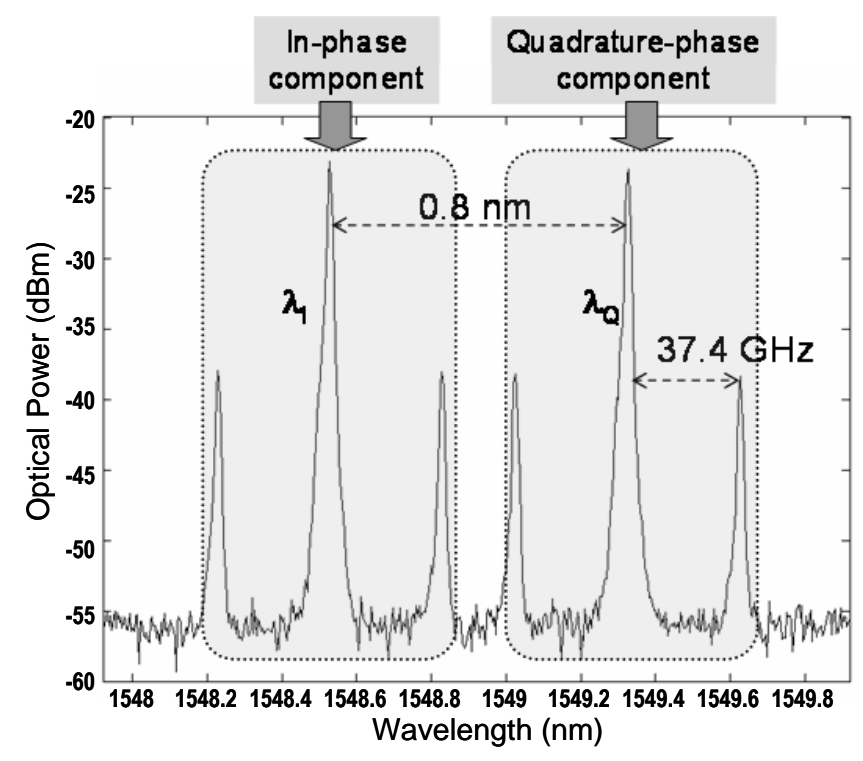

Figure 99. Optical spectrum at the photodiode input.

In the photodiode, these signals are photodetected generating the RF quadrature modulation. The received signal was observed using a digital communication analyser, which displays the signal quality parameters directly at the RF frequencies.

With the proposed architecture, $74.7 \mathrm{Mb} / \mathrm{s}$ QPSK signal at $37.63 \mathrm{GHz}$ was successfully generated with an SNR (actually measured as modulation error ratio (MER)) of $15.7 \mathrm{~dB}$. The digital constellation and the demodulated Q-component eye diagram are depicted below. 

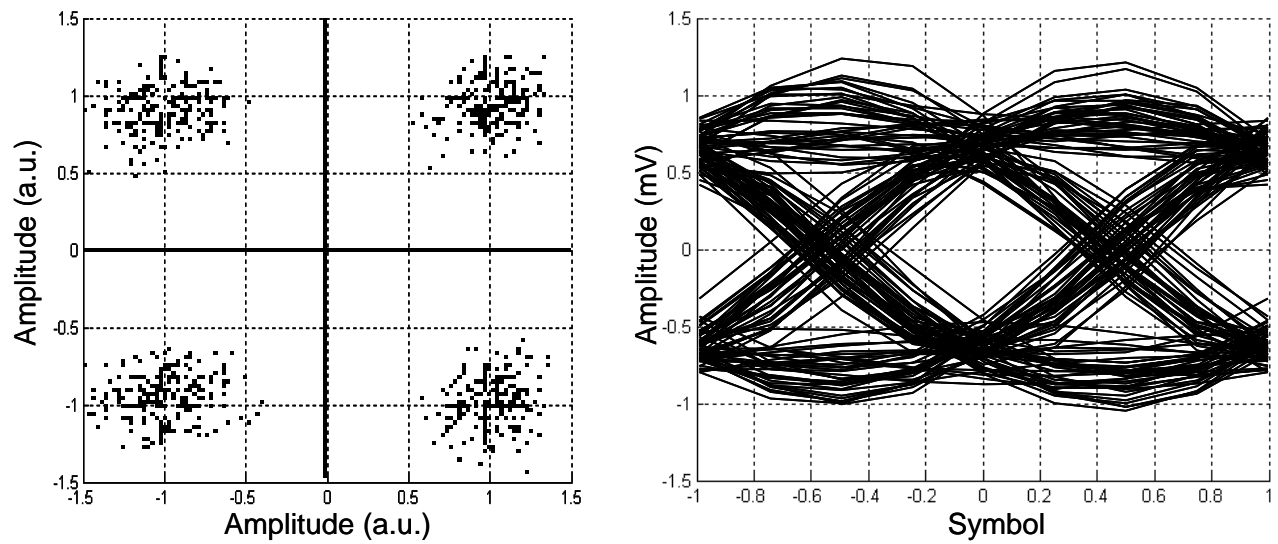

Figure 100. Constellation and quadrature-phas e eye diagram for the ge ne rated QPSK signal.

From the RF spectrum analyser measurement depicted in the inset of Figure 101, it can be seen that no electrical filtering is necessary since harmonic terms are not generated. In the figure, both the unmodulated LO tone and the generated QPSK signal are shown. The shape of the signal is due to the raised root cosine filtering showing a bandwidth of $40 \mathrm{MHz}$.

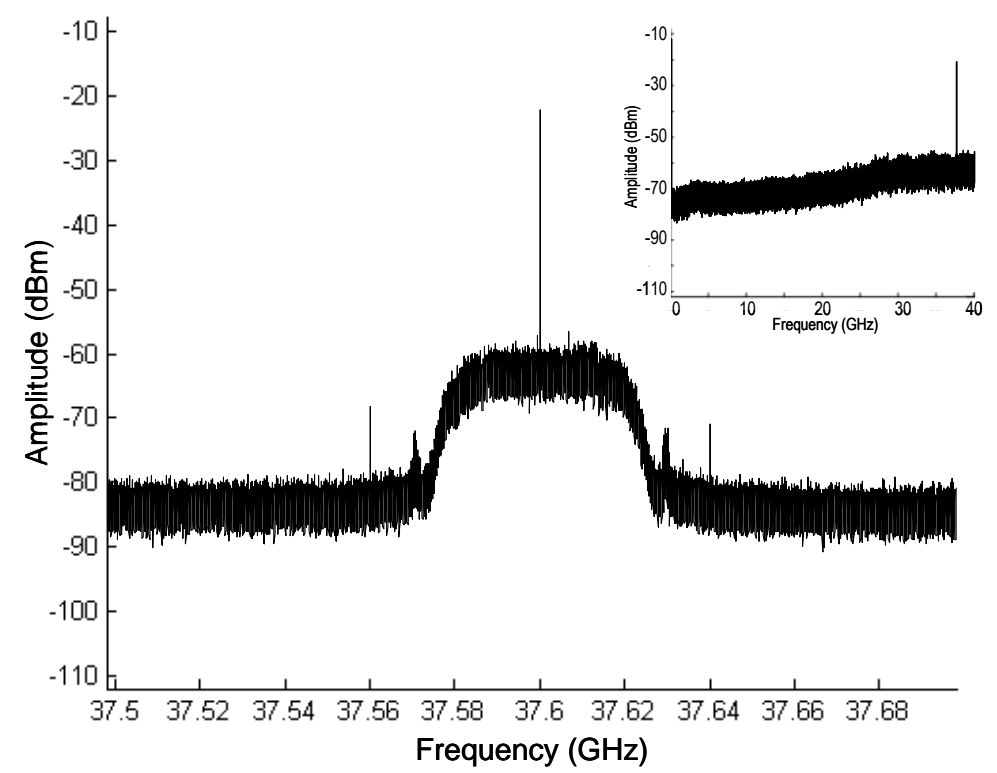

Figure 101. Electrical spectrum of the ge nerated signal at $37.4 \mathrm{GHz}$. Inset: Electrical spectrum at photodiode output from 0 to $40 \mathrm{GHz}$. 
Finally, in order to measure the system tolerance against detuning in the electrical or optical frequencies, some additional tests were conducted. The wavelength spacing was shifted $\pm 0.025 \mathrm{~nm}$ and the local oscillator frequency was tuned in the $37.6 \pm 1.2 \mathrm{GHz}$ range. For all the cases, the generated QPSK signal was demodulated with the digital analyser, obtaining an SNR greater than $14.5 \mathrm{~dB}$ (corresponding with a bit error rate lower than $10^{-9}$ for a QPSK modulation), showing that the generation system is tolerant to changes in the system parameters as shown in Table 20. Therefore, the generated QPSK signal is suitable for transmission in an actual wireless system.

\subsection{Photonic Vector QAM Modulator Architecture}

A photonic quadrature modulator which extends the concept previously proposed employing a unique EOM, a dispersive medium as cheap as optical fibre and baseband modulated optical sources is depicted in Figure 102.

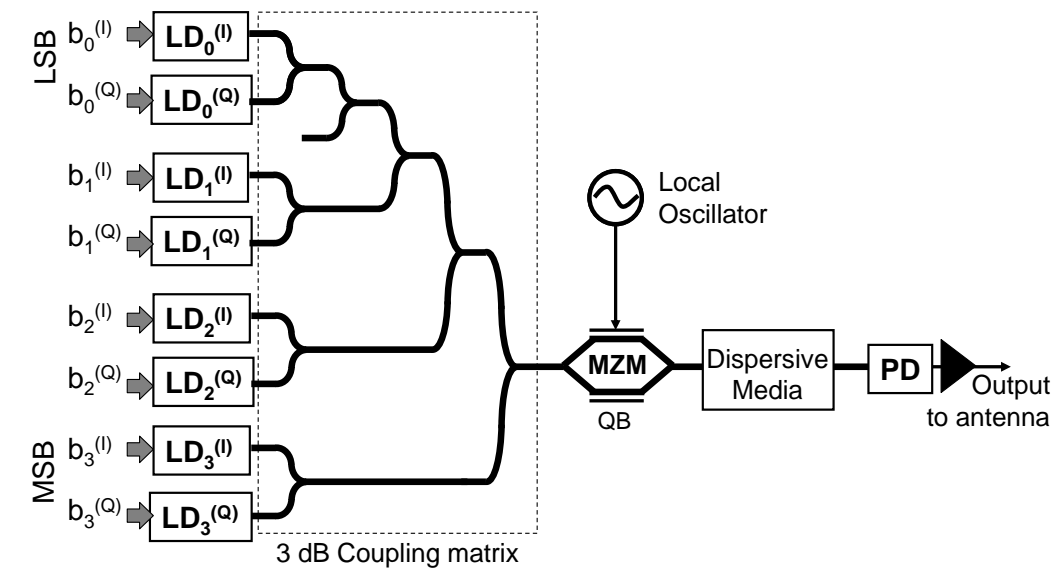

Figure 102. Multilevel Photonic Quadrature-Amplitude-Modulator Architecture.

The data source is a digital word formed by as many bits as required to obtain the desired modulation level. For example, to generate a 64QAM, a digital word of $\log _{2}(64)=6$ bits is needed. This parallel digital stream directly modulates the lasers, which are standard optical sources widely employed, for instance in Gigabit Ethernet systems. To generate the QAM signal, the even bits $\left(b_{x}{ }^{(I)}\right)$ (and lasers) will form the in-phase component, and the odd bits $\left(b_{\mathrm{x}}{ }^{(Q)}\right)$ will form the quadrature one. The optical signals are combined employing cascaded $3 \mathrm{~dB}$ optical couplers. This structure weights the power of each laser (or bit) by a factor of $2^{-n / 2}, n$ being the bit sub index. In the Mach-Zehnder Modulator (MZM), all the binary optical signals are modulated by a local oscillator (LO) at the carrier frequency, resulting in an on-off modulation 
of the LO for each laser. Then, the optical signal is transmitted to the photodiode (PD) over a dispersive medium such as standard single mode optical fibre (SSMF). Due to the chromatic dispersion of the fibre, a differential delay $(\Delta \tau)$ among optical components is introduced as reported in the chapter 1.

By a proper design, this delay can be tuned to be equal to a quarter of the LO period $\left(\Delta \tau=T_{L O} / 4\right)$. This delay represents a phase shift of 90 degrees between the optical envelopes of adjacent wavelengths. At the PD output, as many replicas of the LO with differential phase shift of 90 degrees as bits are obtained. The resulting normalized LO component of the photocurrent at the PD output is:

$$
\begin{aligned}
\frac{\overline{i_{L O}}}{e^{j \omega_{L O} t}} & =\left[b_{0}^{(I)}-\frac{1}{2} b_{2}^{(I)}+\ldots+\frac{1}{(-2)^{N / 2}} b_{N-2}^{(I)}\right] \\
& +j\left[b_{1}^{(Q)}-\frac{1}{2} b_{3}^{(Q)}+\ldots+\frac{1}{(-2)^{N / 2}} b_{N-1}^{(Q)}\right]
\end{aligned}
$$

This signal is a QAM modulation at the LO frequency $\left(\omega_{\mathrm{LO}}\right)$. In fact, each QAM component is a multilevel signal with $2^{N / 2}$ linearly spaced levels, $N$ being the total number of bits.

With the proposed architecture, the QAM signal can be obtained directly from the parallel binary word, which results in a very simple and scalable architecture. In addition, since the multilevel signal is constructed at the PD output, no lasers with special linear modulation performance are required, simplifying the required hardware. The same considerations for carrier tuning, laser wavelength stability or fibre length tolerance stated in the previous section are applicable to this architecture. Finally, this scheme is suitable for remote antenna applications by using dispersion management in the fibre span, and the lasers, coupling matrix and the modulator could be integrated in a single photonic chip.

\subsubsection{Experimental Results}

In the experimental setup, three lasers directly modulated by $1 \mathrm{~Gb} / \mathrm{s}$ NRZ signals, with $1 \mathrm{~V}$ amplitude, were employed in order to generate a $3 \mathrm{~Gb} / \mathrm{s}$ 8QAM signal at the LO frequency $(39.23 \mathrm{GHz})$. The optical signals were polarization controlled and combined in a coupling matrix composed by two cascaded $3 \mathrm{~dB}$ optical couplers. The optical carriers, were centred at 1550.12, 1549.32 and $1548.52 \mathrm{~nm}$ These three signals were modulated by the LO in a $50 \mathrm{GHz} \mathrm{MZM}$, amplified by an Erbium-doped fibre amplifier (EDFA), transmitted over a $0.5 \mathrm{~km}$ of $\operatorname{SSMF}(D=16.7 \mathrm{ps} /(\mathrm{km} \cdot \mathrm{nm}))$ and photodetected. In the following figure the electrical spectrum of the generated 
8QAM signal is depicted. It was demodulated by mixing a phase-adjusted replica of the LO with the signal.

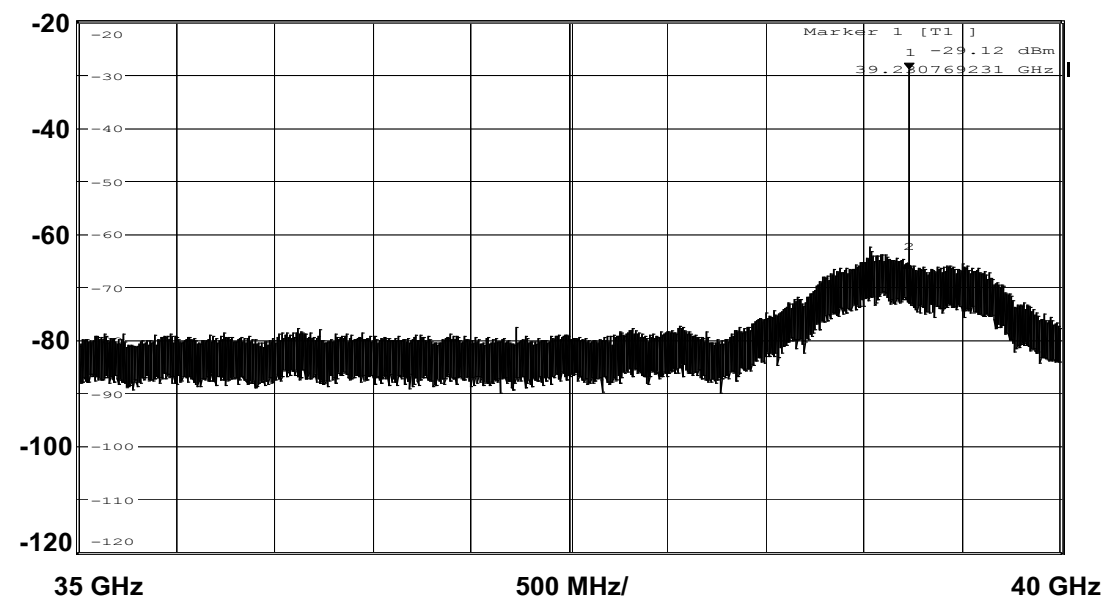

Figure 103. Electrical s pectrum at the photodiode output.
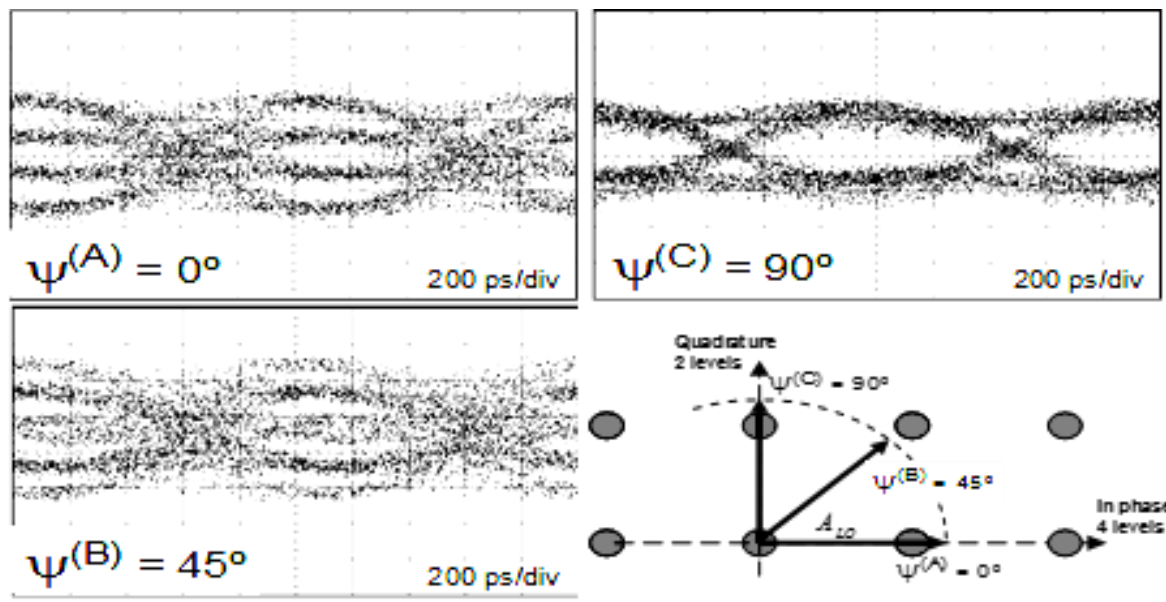

Figure 104. Top: Demodulated eye diagrams of the In-phas e (left) and Quadrature (right) components. Bottom: Intermediate eye (left) and the gene rated $8 \mathrm{QAM}$ constellation diagram (right).

In the previous figure the demodulated eye diagrams of both components, as well as an intermediate eye diagram, are depicted. It can be clearly appreciated the four levels of the In-phase component, and the two of the quadrature one. These measurements were limited mainly by the low level $(1 \mathrm{~V})$ of the modulating binary signals due to the maximum modulation amplitude of the lasers was of $5 \mathrm{~V}$ (TTL standard). 


\subsection{Conclusion}

A photonic quadrature amplitude modulation architectures based on the vector summation technique and fibre dispersion induced delays have been proposed. It can generate from QPSK to more general QAM signals directly at the carrier frequency from parallel binary signals. The required hardware and the modulator control is simple and scalable, especially in the case of the QAM modulator. The fundamental concept of QPSK modulations have been initially demonstrated at low data rates and the more general QAM modulator evaluated by generating a $3 \mathrm{~Gb} / \mathrm{s}-8 \mathrm{QAM}$ signal directly in the $39 \mathrm{GHz}$ band. 


\title{
CHAPTER 9
}

\author{
Simultaneous \\ Base-band and $\mathrm{mm}-$ Wave \\ Delivery of $\mathbf{G b} / \mathbf{s}$ data \\ Employing Photonic Vector \\ Modulators
}

In this chapter, a highly scalable photonic vector modulation (PVM) architecture for the generation of multi-level formats is proposed and experimentally demonstrated. Employing this approach, the generated signals can be detected both at baseband and up-converted to an arbitrary mm-wave frequency, in a highly flexible approach addressing the application scenarios described previously. At baseband, the digital data streams used to compose the multilevel signal can be detected directly after wavelength division demultiplexing the incoming wavelengths. At the same time, the digital data stream is up-converted and remodulated in a multi-level format directly over a RF carrier, which can be amplified, filtered and radiated to a remote location over a wireless link. The experimental demonstration of this concept is reported in this paper, showing the generation of up to $3 \mathrm{~Gb} / \mathrm{s}$ QPSK, 4-ASK and 8QAM signals at $39 \mathrm{GHz}$ and also confirming the capability of transmitting the original data streams at baseband, extending the results provided in the previous chapter. 


\subsection{Scenario Description}

Broadband wireless access transmission at high frequencies is associated with both advantages and disadvantages. High propagation attenuation at $60 \mathrm{GHz}$ actually classifies a set of short-range applications, but it also means dense frequency reuse patterns. Higher frequencies lead to smaller sizes of RF components including antennas. At mm-wave frequencies, not only are the antennas very small, but also they can be quite directional (coming with high antenna gain), which is highly desired.

Three different application scenarios have been identified within this chapter. The different scenarios for the proposed millimeter wave $(\mathrm{mmW})$ wireless architecture are depicted in the following figures. In Figure 105, the Fixed Wireless Access (FWA) interconnection of networks nodes at high data rates (up to $10 \mathrm{~Gb} / \mathrm{s}$ ) is depicted. The solution provides a fixed wireless access that would make trenching difficult or impossible and so significantly increase the cost for a direct fiber link, e.g. over highways, rivers or other nature obstacles. Figure 106 illustrates the coverage extension via wireless of the FTTH network, a concept known as FiberTo-The-Air (FTTA). Finally, in Figure 107 the concept of transmission of uncompressed HDTV signal is shown. These three scenarios have a clear market; covering from the fast access to remote areas in FTTH networks (as either as temporal or permanent solution, reducing the CAPEX and the time-to-market) to backup solutions based on the wireless redundancy, including disaster recovery solutions. Most of these scenarios are identified by a solid fast uplink; however, some scenarios need both an upstream and downstream link which can be achieved by exploiting the polarization properties of the $\mathrm{mmW}$, doubling the available bandwidth.

\subsubsection{Application Scenario 1: Point-to-point Fixed Wireless Access (FWA) connections}

Point-to-point FWA application is a desired solution for a handful of scenarios, spanning from core network island connections to disaster recovery and backup solutions. LAN and network connections need a bidirectional connectivity; however, backup solution for data centers functions with one fast and reliable upstream link. The five most interested scenarios have been identified and are given in bullet form:

- Island network connectivity. Carriers can deploy mmW wireless links to extend existing metropolitan-area fiber rings, to connect new networks, and, in their core infrastructure, to extend ETHERNET connectivity.

- LAN connections (buildings, urban areas, enterprise connectivity). The ease with which $\mathrm{mmW}$ links can be installed makes them a potential solution for 
interconnecting local-area network segments that are housed in buildings separated by public streets or other right-of-way property.

- Disaster recovery. mmW links may also be deployed in redundant links to back up fiber in place of a second fibre link.

- Service acceleration. mmW can be also used to provide instant service to fiber-optic customers while their fiber infrastructure is being laid.

- Mirror Data Center real time wireless backup connections (for example, for banking entities). Baseband and sideband from same transmitter.

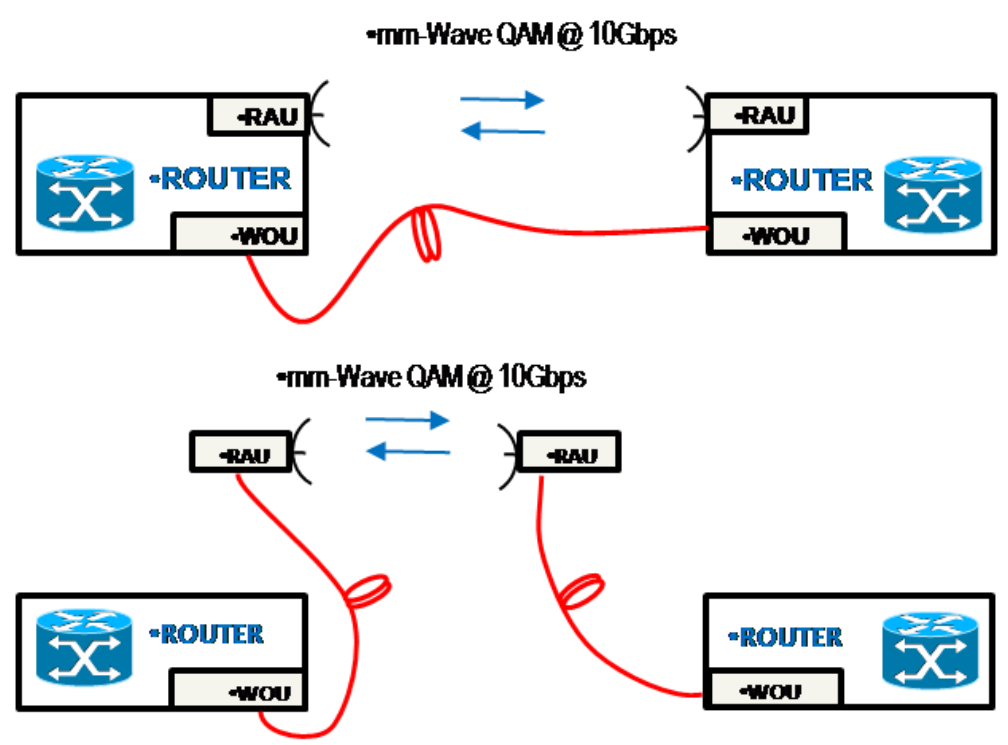

Figure 105. High-speed point-to-point FWA Application Scenario. (upper) A real-time wire less backup link. (lower) Point-to-point FWA connection.

\subsubsection{Application Scenario 2: Coverage extension via wireless of fibre-to-the-home}

In many cases the extension of fiber to the last mile is challenging, especially in rural areas, hilly regions etc, where these $\mathrm{mmW}$ wireless links can be used to provide gigabit connectivity to the user. Some examples are hereby provided:

- Last mile coverage of FTTH.

- Last-mile access mmW can be used in high-speed links that connect end-users with Internet service providers or other networks. It can also be used to bypass local-loop systems to provide businesses with high-speed connections.

- Fast deployment and revenue and flexibility. Reduced time-to-market. 


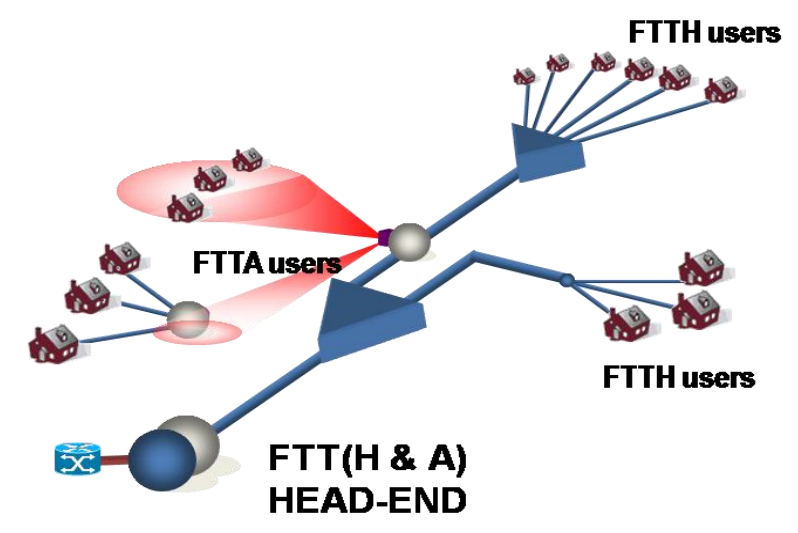

Figure 106. Fibre-to-the-air application s cenario.

\subsubsection{Application Scenario 3: Uncompressed HD video transmission}

Short distance uncompressed HD video transmission and HD multimedia interface are feasible technologies using a few $\mathrm{Gb} / \mathrm{s} \mathrm{mmW}$ wireless systems. Sports events as well as HD multimedia homes have beenidentified as promising scenarios. As an example, in Japan, NTT and Fuji TV are deploying $10 \mathrm{~Gb} / \mathrm{s}$ wireless links for direct uncompressed transmission of HDTV in a stadium. These four scenarios have been identified in this thesis:

- Uncompressed HD video short range broadcasting transmission system.

- HDTV with mmW Video-Signal receiver.

- mmW Ad-Hoc Wireless Access System.

- Multimedia Home Link.

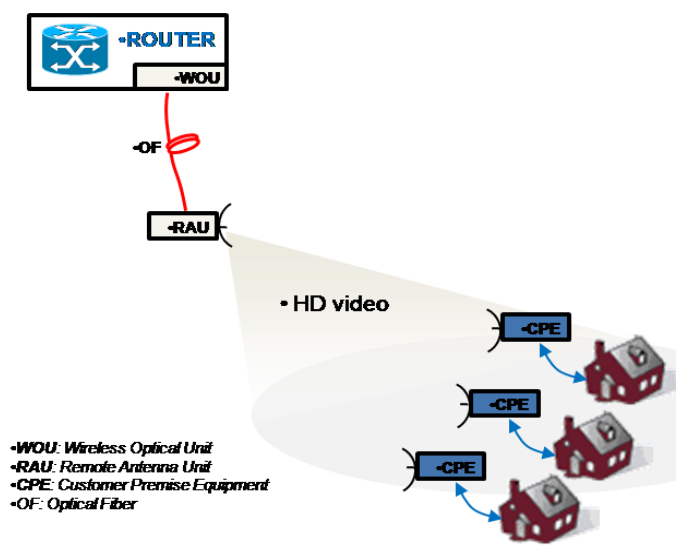

Figure 107. Uncompressed HD video signal trans mission application scenario. 


\subsection{Architecture Description and Experimental Setup}

Figure 108 shows the setup of the proposed PVM system to provide wireless $\mathrm{Gb} / \mathrm{s}$ multi-level modulated signals with simultaneous transmission of baseband signals. The operating principle is based in vector summation of the amplitudes and phases of differentially delayed modulated wavelengths in the photodetection process. The data source is a digital word formed by as many bits as required to obtain the desired modulation level, for instance a digital word of $n=6$ bits is required to generate a 64QAM modulation. This parallel digital streamdirectly modulates the lasers that can be standard optical sources.

To generate the QAM signal, the even bits $\left(b_{x}{ }^{(I)}\right)$ (and lasers) will form the inphase component, and the odd bits $\left(b_{x}^{(Q)}\right)$ will form the quadrature one. The optical signals are combined employing cascaded $3 \mathrm{~dB}$ optical couplers. In the Mach-Zehnder Modulator (MZM), all the modulated optical carriers are modulated by a local oscillator (LO) tone at the carrier frequency. Then, the optical signal is transmitted to the photodiode (PD) over a dispersive medium such as standard single mode optical fibre (SSMF). that performs the 90 degrees phase shift for the quadrature modulation as described in the previous chapter. Employing the proposed architecture is possible to generate other modulation formats such as QPSK or 4ASK, by simply adjusting the phase relationship between the optical carriers by properly tuning the differential delay.

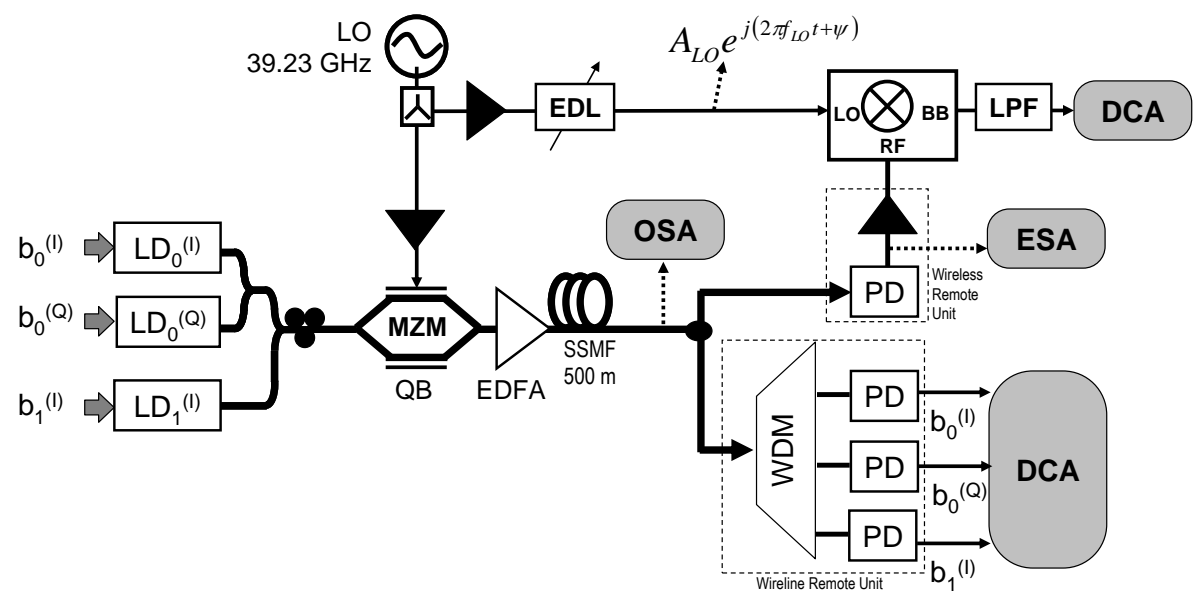

Figure 108. Schematic of the proposed PVM architecture and phase-matched demodulation arrangement employed in the experiments. 


\subsection{Experimental Measurements}

The setup depicted at Figure 108 was employed to validate the proposed approach. A set of three lasers directly modulated by high speed NRZ signals with $1 \mathrm{~V}$ amplitude, were employed in order to generate up to $2.5 \mathrm{~Gb} / \mathrm{s}$ 4ASK, QPSK and $3 \mathrm{~Gb} / \mathrm{s}$ 8QAM signals at the LO frequency $(39.23 \mathrm{GHz})$. Only two lasers are employed in order to generate 4ASK and QPSK signals, whilst all three are needed to generate the 8QAM modulation format. In all cases, the optical signals were polarization controlled and combined in a coupling matrix composed by two cascaded $3 \mathrm{~dB}$ optical couplers. The optical carriers, were centred at 1550.12, 1549.32 , and $1548.52 \mathrm{~nm}$. The corresponding optical signals were modulated by a $+15 \mathrm{dBm}$ LO signal in a $50 \mathrm{GHz}$ Mach-Zehnder modulator (MZM) biased at the quadrature point, amplified by an Erbium-doped fibre amplifier (EDFA), transmitted over a $0.5 \mathrm{~km}$ of $\operatorname{SSMF}(D=16.7 \mathrm{ps} /(\mathrm{km} \cdot \mathrm{nm}))$ and photodetected. The optical power at the input of the PD was $+2.5 \mathrm{dBm}$. In Figure 109 (left) the electrical spectrum of the generated signals, measured with an electrical spectrum analyser (ESA) is depicted, where both the baseband data and the up-converted signal at $39.23 \mathrm{GHz}$ can be clearly distinguished. As shown in Figure 108, the multi-level RF carrier was demodulated by mixing a phase-adjusted replica of the LO with the signal. Figure 109 (right) shows the eye diagram of the $2.5 \mathrm{~Gb} / \mathrm{s}$ down-converted signal for the case of 4ASK modulation measured using a digital communications analyzer (DCA). It should be noticed that, in this case, an envelope detector can be used at the receiver in a real implementation, avoiding the need of wideband electrical mixers and phase-locked loops.
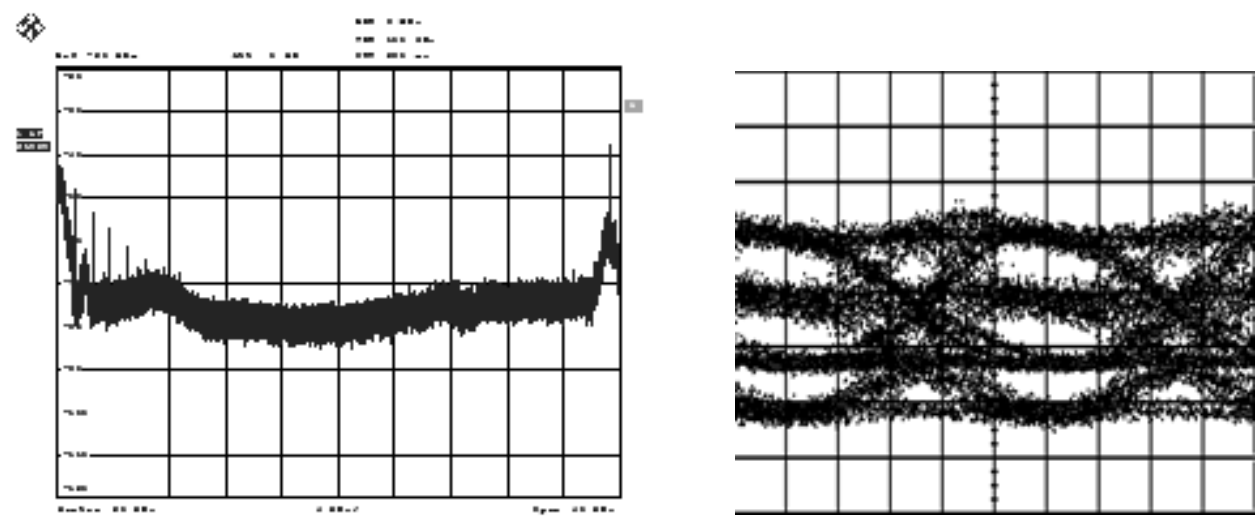

Figure 109. Measured e lectrical spectrum at PD output (left) and detected $2.5 \mathrm{~Gb} / \mathrm{s} 4$-ASK signal down-converted from $39.23 \mathrm{GHz}$ (right).

To demonstrate the feasibility of simultaneous recovery of the baseband data, the set-up was used to generate a $3 \mathrm{~Gb} / \mathrm{s} 8 \mathrm{QAM}$ signal. The three wavelengths were 
demultiplexed and photodetected. The measured eye diagrams of each parallel bit stream are shown in Figure 110. Q parameters of 11.4, 11.2 and 10.4 where estimated by the DCA software, respectively. These bit streams can be serialized in order to get the original $3 \mathrm{~Gb} / \mathrm{s}$ bit stream or fed directly to an optical switch that can handle the incoming parallel data streams directly in the optical domain.

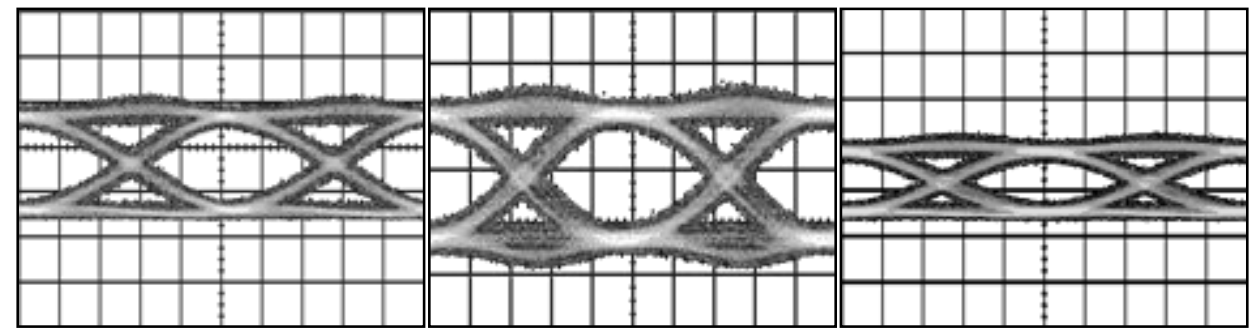

Figure 110. Measured eye-diagrams of $1 \mathrm{Gr} / \mathrm{s}$ parallel bit streams after demultiplexing measured by the DCA: $b_{0}{ }^{(\mathrm{I})} @ 1550.12$ nm (left), $b_{0}{ }^{(Q)} @$ $1549.32 \mathrm{~nm}$ (mid), $b_{1}{ }^{(\mathrm{I})} @ 1548.52 \mathrm{~nm}$ (right). Horizontal scale is $200 \mathrm{ps} /$ and vertical scale is $20 \mathrm{mV} /$.

The proposed PVM architecture is a highly flexible approach to provide broadband data simultaneously at base-band and at RF frequencies. If dispersion management is included, to cope with differences in fibre spans within the network, the proposed arrangement will find application in real scenarios such as that described previously.

The generation of multi-level modulations directly at mm-wave frequencies employing photonic vector modulator schemes has been demonstrated. QPSK, 4ASK and 8QAM modulations formats have been obtained experimentally with data rates up to $3 \mathrm{~Gb} / \mathrm{s}$ in the $39 \mathrm{GHz}$ frequency band. Moreover, the feasibility of deploying multi-Gb/s signals simultaneously at baseband and $\mathrm{mm}$-wave frequencies has been demonstrated, in a highly flexible approach. Since the multilevel signal is constructed at the PD output, no lasers with special linear modulation performance are required, simplifying the photonic components requirements. The same considerations for carrier tuning, laser wavelength stability or fibre length tolerance stated in the previous sections are applicable to this architecture. Finally, this scheme is suitable for remote antenna applications by using dispersion management in the fibre span, and the lasers, coupling matrix and the modulator could be integrated in a single photonic chip. 


\subsection{Photonic Integrated Circuit implementing a Heterodyne Photonic Vector Modulator}

This section focuses on the development of a photonic $10 \mathrm{~Gb} / \mathrm{s}$ wireless transmission system, with a high spectral efficiency. A novel concept of heterodyne wireless signal generation in the mmW band in conjunction with DQPSK photonic modulation scheme will be described.

The millimetre-wave transmitter and receiver are based on optical communication technology involving DQPSK modulation formats. In this system, the millimetre wave signal is first generated optically, which can be either using heterodyne technique in a Mach-Zehnder modulator or using a dual mode laser. In both the cases, two coherent optical signals separated by the radio frequency $\left(f_{L O}\right)$ to be emitted are generated. The two optical carriers are divided using an optical filter which can be based on different technologies like MZI, AWG, etc. One of the optical carriers is now phase modulated in a DQPSK format using a dual-parallel MZM as proposed in [GRI02]. In this modulation format the phase of the optical signal is modulated by the electrical data resulting in four different phase levels corresponding to I and Q data. The modulated optical carrier is combined with the other unmodulated optical carrier separated by the RF frequency. When the combined optical signals are mixed in a photodetector, the output of the photodetector is a millimetre wave carrier with the I/Q data modulated in a (D)QPSK format at the carrier frequency of $f_{L O}$. It should be noted that the optical DQPSK modulation format is a phase only modulation performed by MZMs biased in push-pull mode, and no amplitude modulation is induced except for an amplitude dip for every transition between a bit 0 and bit 1 . Figure 111 shows the schematic of the transmitter. This design enables the simultaneous generation of optical base band and millimeter-wave modulations as the architecture described in the previous section but in this case integrated in a single photonic chip. 


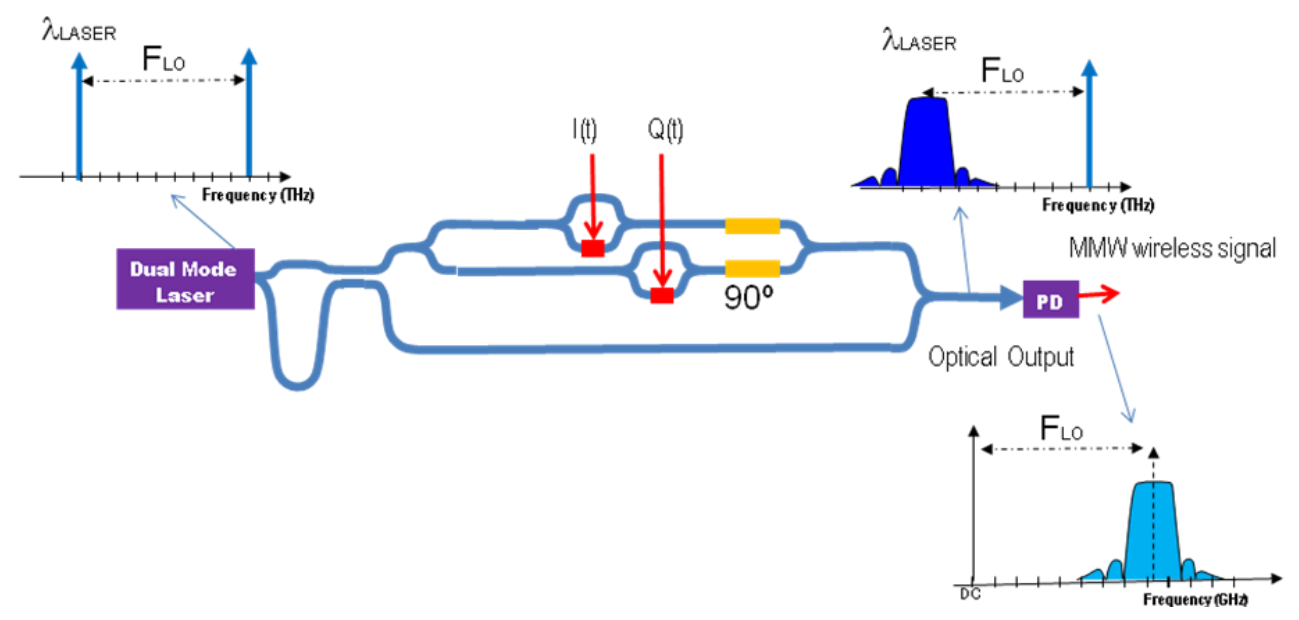

Figure 111. Schematic of the mmW transmitter.

\subsubsection{Mathematical Model}

Let the output of the dual mode laser be:

$$
E(t)=\sqrt{\frac{P}{2}}\left[\exp j\left(\omega_{0} t\right)+\exp j\left(\left(\omega_{0}+\omega_{L O}\right) t\right)\right]
$$

Assuming ideal filtering and DQPSK modulation of the optical carrier $\left(\omega_{0}\right)$ and $\left(\omega_{0}+\omega_{L O}\right)$ where $\omega_{L O}$ is the carrier frequency, the optical output of the modulator is expressed as

$$
E_{\text {out }}(t)=\sqrt{\frac{P}{2 t_{f f}}}\left[\frac{1}{\sqrt{L_{1}}} \cos \left(\frac{\pi\left(I_{k}-Q_{k}\right)+\frac{\pi}{2}}{2}\right) \mathrm{e}^{j\left(\omega_{0} t+\frac{\pi\left(I_{k}+Q_{k}\right)+\frac{\pi}{2}}{2}\right)}+\frac{1}{\sqrt{L_{2}}} \mathrm{e}^{j\left(\omega_{0}+\omega_{L O}\right) t}\right]
$$

where $t_{f f}$ is the insertion losses, $L_{1}$ and $L_{2}$ are the losses induced in each arm.

The photo current is expressed in the following equation:

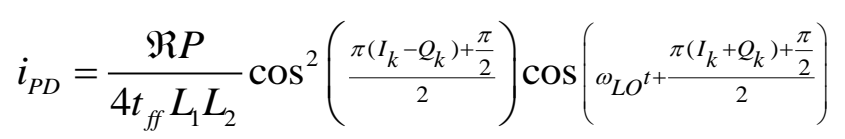

where $\mathfrak{R}$ is the responsivity of the photodetector. 
A photonic receiver is incorporated to demodulate the wireless signals. The received wireless signal is first modulated on an optical carrier $\omega_{l}$ using another external modulator typically MZM. The output of the MZM is described as follows:

$$
\begin{aligned}
E_{R X-\text { out }} & =\sqrt{\frac{2 P_{1}}{L_{R X}}}\left[J_{0}\left(m_{R F}\right)+J_{1}\left(m_{R F}\right) \cos \left(\omega_{L O^{t+}} \frac{\pi\left(I_{k}+Q_{k}\right)+\frac{\pi}{2}}{2}\right)\right] \mathrm{e}^{j \omega_{1} t} \\
& =\sqrt{\frac{2 P_{1}}{L_{R X}}}\left[J_{0}\left(m_{R F}\right) \mathrm{e}^{j \omega_{1} t}+J_{1}\left(m_{R F}\right) \mathrm{e}^{j\left(\left(\omega_{1} \pm \omega_{L O}\right) t \pm \frac{\pi\left(I_{k}+Q_{k}\right)+\frac{\pi}{2}}{2}\right)}\right]
\end{aligned}
$$

where $m_{R F}=\frac{\pi V_{R F}}{V_{\pi}} \cos ^{2}\left(\frac{\pi\left(I_{k}-Q_{k}\right)+\frac{\pi}{2}}{2}\right), L_{R X}$ are the insertion losses of the MZM. $\mathrm{V}_{R F}$ is the voltage of the RF signal and $V_{\pi}$, the voltage required for the MZM to obtain a 180 degrees phase shift.

From the expression (9.4) it can be observed that the output of the MZM contains the optical carrier $\omega_{1}$ and the two sidebands which contain the phase modulated data. Using an optical bandpass filter and filtering the upper sideband results again in an optical baseband signal with the I/Q data modulated in DQPSK format. It is worth mentioning that the wireless link has maintained the differential phase information intact. Also the demodulation process does not involve any kind of phase coherence of the RF carrier which is normally the case for vector modulated RF signals. The system is also robust to the phase noise of the laser at the transmitter because of differential detection of the phase, where the information is coded in the phase difference and not in the absolute phase. To perform direct detection of the baseband DQPSK signal a 1-bit delay interferometer is used which converts the phase information to amplitude. Typical DQPSK receivers consist of two 1-bit delay interferometers with phase offsets of $+/-\pi / 4$. The optical signal entering one of the DMZI is equally split into arms where one of the arms induces a delay equal to 1-bit period, and the other arm a phase offset. Figure 112 shows the schematic of the receiver and the DMZI. 


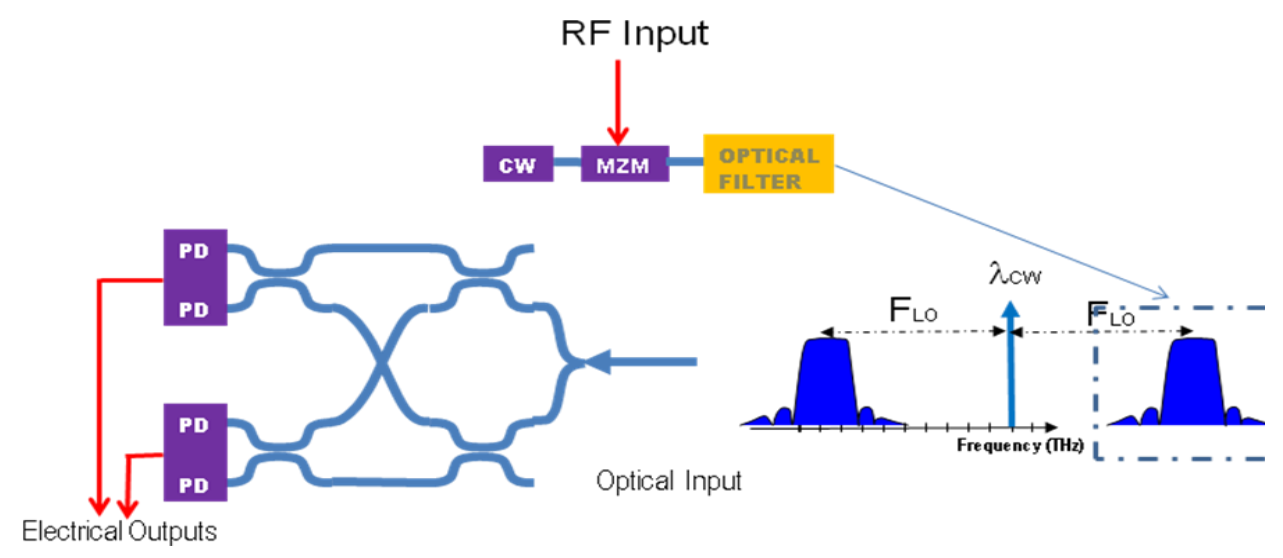

Figure 112. Schematic of the re ceiver for de modulation of the wire less signals.

\subsubsection{Discussion}

The two PIC designs described above implementing either a modulator and a demodulator of quadrature signals is required to be manufactured in a photonic platform that enables the high speed modulation and detection, which should be InP or CMOS Photonics. The activities develop in this matter within this thesis included the generation of the fundamental idea, the conceptual design and the preparation of a patent to protect the property. After this, this concept was proposed in the European FP7 project HELIOS in order to be implemented in CMOS photonics. These tasks were conducted by my colleagues Dr. Andreas Hakansson and specially Dr. Rakesh Sambaraju who conducted his $\mathrm{PhD}$ in the topic of Photonic Vector Modulation. 


\title{
ChAPTER 10
}

\author{
Photonic \\ Vector Demodulation \\ Architecture for Remote \\ Detection of M-QAM \\ Signals
}

A photonic vector demodulation $(\mathrm{PVdM})$ architecture for the reception of QAM signals at microwave/mm-wave band is presented in this chapter. With this concept no electrical devices that virtually limit the useful bandwidth and the carrier frequency are employed exploiting the advantages of the photonic technology as the efficient optical remote detection or huge electrical bandwidth. Finally, to show the feasibility of the architecture, preliminary results are provided.

\subsection{Architecture Description}

A schematic view of the system and the demodulation process can be seen in Figure 113 and Figure 114, respectively. The system consists of two optical sources, emitting at wavelengths $\lambda_{1}$ and $\lambda_{2}$. The optical signals are combined, modulated by a microwave frequency tone in an external quadrature biased (QB) Mach-Zehnder modulator (MZM) (point 1 at Figure 114) and transmitted over a standard single-mode fibre (SSMF). Since MZMs are optical-polarization dependent devices, polarization controllers or polarization-maintaining fibre must be employed. The 
fibre chromatic dispersion induces a delay between both electrically modulated optical signals equal to $\Delta \tau=D \cdot L \cdot \Delta \lambda$, where $\Delta \lambda=\lambda_{2}-\lambda_{1}$ is the optical wavelength spacing. This delay can be tuned by changing the fibre length or the wavelength spacing in order to obtain a delay of a quarter of the microwave LO tone period (quadrature condition), which corresponds with a differential phase $\left(\phi_{\mathrm{D}}\right)$ of $\pi / 2$ radians between both optical signals. The dispersion delaying process has been illustrated in points 2 and 3 of Figure 114 corresponding with an intermediate point and the final point of the optical link. The total induced delay to the optical envelope at point 3 is $\phi_{D}=\pi / 2$ radians.

Then, the optical signals are again electrically modulated by a QAM signal in a second MZM, being its central frequency equal to the LO frequency, named $f_{L O}$. The electrical QAM modulation, composed by the in-phase (I) and quadrature (Q) components, is illustrated in point 4 of the Figure 114. The resulting signal at the second MZM output corresponds with two optical carriers simultaneously modulated with an electrical carrier and a QAM signal, both with the same central frequency. With an appropriated tuning, the phase of each $\mathrm{LO}(\pi / 2$ radians delayed $)$ and I and Q components can be equalized as can be seen in point 5 at figure 2 . Using one wavelength optical demultiplexer (WDM) both optical carriers can be separated and independently photodetected in two photodiodes (PD). The non-linear photodetection process generates a down-conversion of the QAM signal from $f_{L O}$ frequency to baseband. Since the appropriated phase adjusting, previously mentioned, only one of the two component (in-phase or quadrature) of the QAM signal is down-converted in each PD achieving the QAM demodulation (points 6 and 7 of Figure 114).

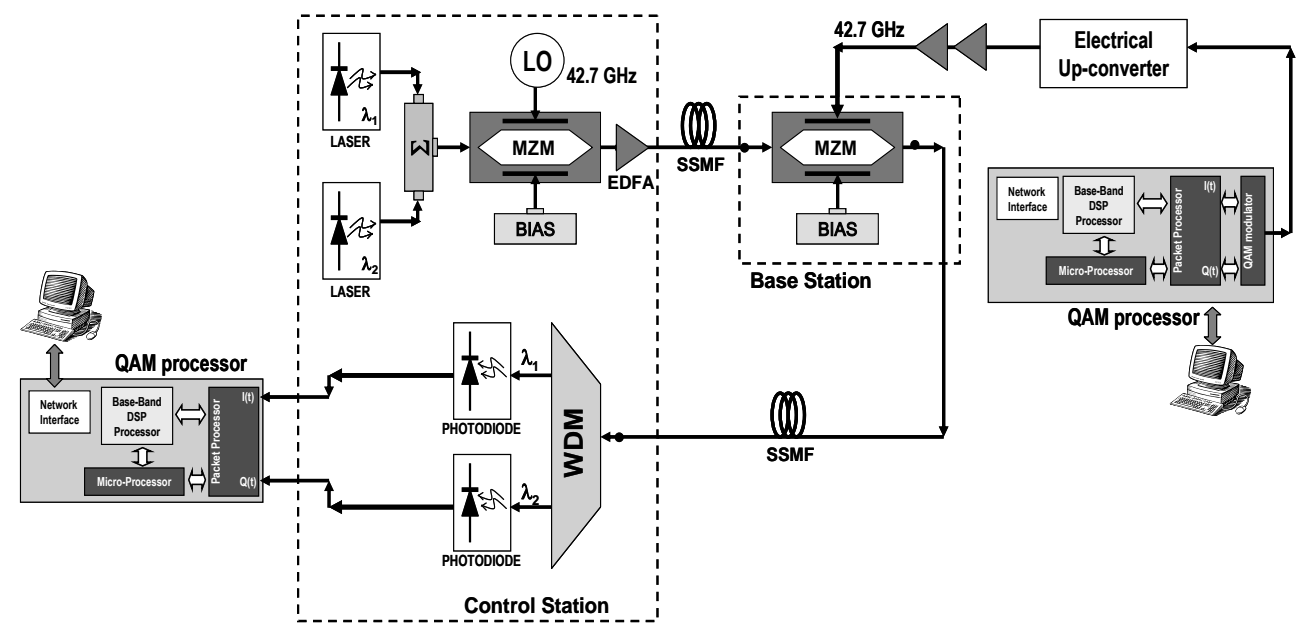

Figure 113. Photonic vector demodulator architecture. 
The photoreceived current in any In-phase or Quadrature arms is:

$$
I_{I, Q}=k J_{0}\left(m_{L O}\right) J_{1}\left(m_{L O}\right) \cdot I, Q
$$

where $k$ is a constant that depends on the system parameters, as electrical and optical losses, photodiode responsivity, etc. $J_{0}$ and $J_{l}$ are the zero-th and first order Bessel functions of the first kind, respectively, and $m_{L O}=\pi V_{L O} / 2 V_{\pi}, V_{L O}$ being the amplitude of the local oscillator tone and $V_{\pi}$ the MZM half wave voltage.
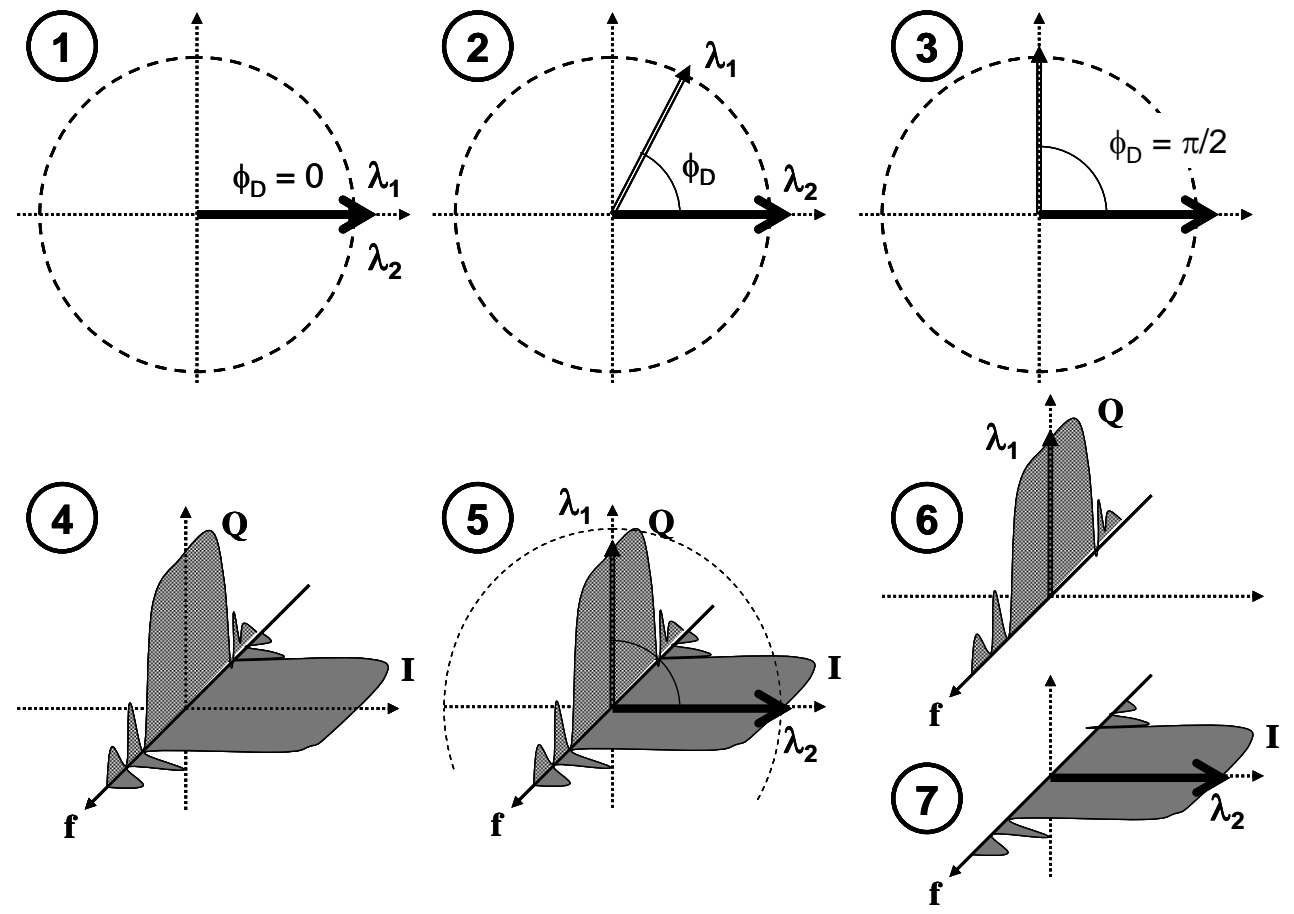

Figure 114. Photonic demodulation process concept.

Finally, the obtained baseband I and Q components are processed in baseband in order to obtain the digital information of this analogue signal. In this scheme, no electrical devices that introduce complexity and impose bandwidth limitations, such as hybrids, combiners or filters, are needed in the demodulation process.

In order to include remote delivery capabilities, the second MZM can be sited in a remote base station (BS) and the remaining devices at a control station (CS) to obtain a very simplified BS scheme. Also, a fibre link between the second MZM and the WDM can be included since this optical fibre will not affect to the detection process. On the other hand, the system can be designed to overcome the restriction imposed by the fact that a fixed link distance between both MZM's is necessary for 
a given LO frequency and wavelength separation. Actually, in realistic scenarios, the distance between the control station (CS) and base stations (BS) is not fixed and it must be covered by the fibre link. The solutions for this issue can be tuning the laser wavelength separation in order to maintain the $D \cdot L \cdot \Delta \lambda$ product constant, using one fix and other tuneable optical source.

\subsection{Performance Analysis}

The proposed system must be adjusted for a given local oscillator frequency. This adjustment is realized by tuning the optical fibre length and the wavelength separation between lasers for a given dispersion parameter. Therefore, tuning errors will produce a system performance penalty, in particular, a deviation in the received LO phases that moves they away from the quadrature state. Considering a maximum received LO phase deviation of 1 degree as performance limit, the tolerances in the fibre length and wavelength separation for different $\mathrm{LO}$ frequencies are depicted in Table 21 considering a fibre dispersion parameter of $\mathrm{D}=17 \mathrm{ps} /(\mathrm{km} \cdot \mathrm{nm})$.

As can be seen in Table 21, a high margin in the tuning parameters exists (for example, for a $60 \mathrm{GHz} \mathrm{LO}$, a margin of 0.9 meters in fibre length) concluding that the proposed architecture is easily adjusted.

TABLE 21: SYS TEM TOLERANCES VS. LO FREQUENCY.

\begin{tabular}{|c|c|c|c|c|}
\hline$f_{L O}(\mathbf{G H z})$ & 5 & 10 & 40 & 60 \\
\hline$\delta \mathbf{L}(\mathbf{m})$ & 10.2 & 5.1 & 1.3 & 0.9 \\
\hline $\begin{array}{c}\delta \Delta \lambda(\mathbf{p m}) \\
(\Delta \lambda=\mathbf{8 0 0} \mathbf{~ p m})\end{array}$ & $35.6 \mathrm{pm}\left(\equiv 4.4 \mathrm{GHz} @ \lambda_{\mathrm{o}}=1550 \mathrm{~nm}\right)$ \\
\hline $\begin{array}{c}\delta \Delta \lambda(\mathbf{p m}) \\
(\Delta \lambda=\mathbf{3 2 0 0} \mathbf{p m})\end{array}$ & \multicolumn{3}{|c|}{$8.9 \mathrm{pm}\left(\equiv 1.1 \mathrm{GHz} @ \lambda_{\mathrm{o}}=1550 \mathrm{~nm}\right)$} \\
\hline
\end{tabular}

${ }^{(*)}$ Dispersion parameter considered: $\mathrm{D}=17 \mathrm{ps} / \mathrm{km} \cdot \mathrm{nm}$

TABLE 22: LO BANDWIDTH VS . LO FREQUENCY.

\begin{tabular}{|c|c|c|c|c|}
\hline$f_{L O}(\mathbf{G H z})$ & 5 & 10 & 40 & 60 \\
\hline $\begin{array}{c}\text { Bandwidth (MHz) } \\
(\text { Phase error }= \pm \mathbf{1} \text { deg) }\end{array}$ & 110 & 220 & 890 & 1330 \\
\hline
\end{tabular}

${ }^{(*)}$ Dispersion parameter considered: $\mathrm{D}=17 \mathrm{ps} / \mathrm{Km} \cdot \mathrm{nm}$

(**) Calculations for quadrature modulation point 
Other key issue is to know the LO frequency band in which the system is useful. Again, if we employ the 1 degree phase detuning criterion, we can calculate the margin in which the LO frequency can be varied without penalty in the system performance. The resulting bandwidths are depicted in Table 22 for different LO frequencies. From these calculations, it can be seen that the proposed architecture exhibits a LO bandwidth greater than $1 \mathrm{GHz}$ in the $60 \mathrm{GHz}$ band. Then, the system is suitable for its use in standard multichannel applications.

\subsection{Simulation Results}

In order to validate the previously proposed architecture, some simulations have been carried out using the commercial simulation software pack 'Virtual Photonics'. All the architecture, in both transmitting and receiving modes, has been simulated for a local oscillator frequency of $1.5 \mathrm{GHz}$ and for 64QAM signal generation/reception that corresponds with 8 levels baseband input signals $(\mathrm{I}(\mathrm{t})$ and $\mathrm{Q}(\mathrm{t}))$. The simulated block diagram is depicted in Figure 115. 


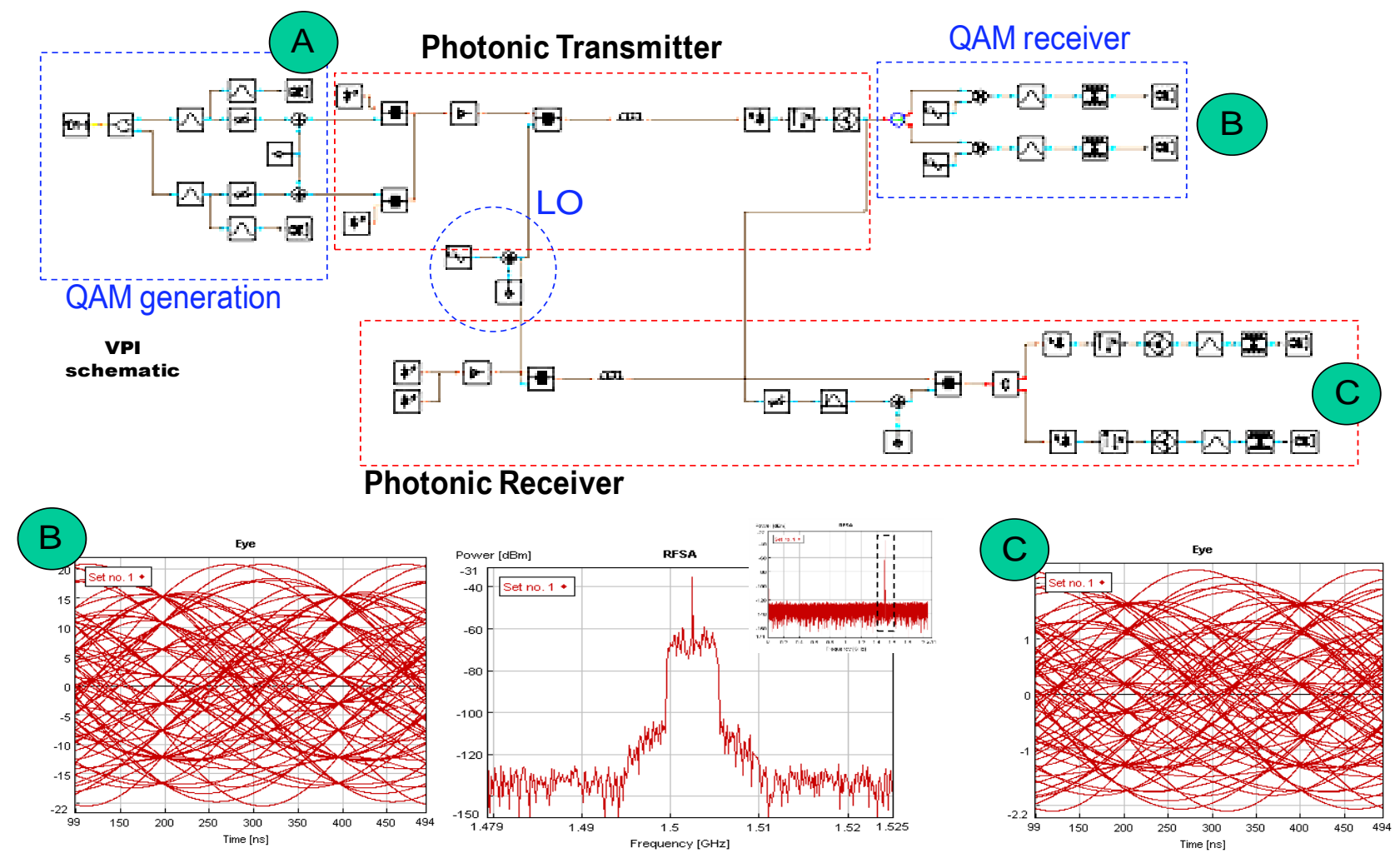

Figure 115. VPI simulation block diagram. Simulate d electrical spectrum at photonic QAM transmitter output. Received 64QAM in-phase component eye diagrams using standard electrical demodulation (left) and the proposed photonic QAM receiver (right). 
In simulations, the same LO has been used in both transmitting and receiving schemes in order to avoid frequency mismatch. The electrical spectrum at point (A) corresponding with the generated QAM signal is depicted in Figure 115 (centre), where it can be seen how the QAM signal is effectively generated at LO frequency band without other passband beats, eliminating the need of microwave filters.

From Figure 115, it can be seen how, effectively, one tone appears at the LO frequency in the generated signal. In Figure 115, eye diagrams for received signals at points (B) (standard electrical reception) and (C) (using the proposed photonic receiver) are depicted. Analogous results were obtained for the quadrature component. It can be observed how in both cases the 64QAM signal is recovered with good quality. In the simulations, the DC level has been eliminated using DC blocks, cancelling the influence of the down-converted LO tone.

Simulations results show a very low penalty in the signal quality for phase detuning of more than 1 degree validating the previous assumption in the tolerance and bandwidth estimations.

\subsection{Experimental Results for QPSK modulations}

In this section, preliminary experimental results that represent a proof-of-concept of the proposed architecture are summarized. Although these results are very preliminary, they confirm that the proposed architecture is suitable to be implemented.

The experimental setup employed for system validation consists in two continuous wave DFB optical sources with a, optical spacing of $1.6 \mathrm{~nm}$, externally modulated by a $42.7 \mathrm{GHz}$ local oscillator tone using a MZM with a $\mathrm{V}_{\pi}$ of $4.7 \mathrm{~V}$. The optical signals are combined in a $3 \mathrm{~dB}$ optical coupler and transmitted over a SSMF link of 240.4 meters with a dispersion parameter of $16.6 \mathrm{ps} /(\mathrm{km} \cdot \mathrm{nm})$. Then, the optical signal is again modulated in a second MZM by a $18.678 \mathrm{Mb} / \mathrm{s}$ QPSK signal, with a raised root cosine filtering and a 0.15 roll-off factor, electrically up-converted to $42.7 \mathrm{GHz}$ frequency band. This data signal was generated by a digital generator (INTERSIL evaluation tool) at a $1 \mathrm{GHz}$ intermediate frequency. The electrical 42.7 GHz up-converted signal spectrum is depicted in Figure 116. 


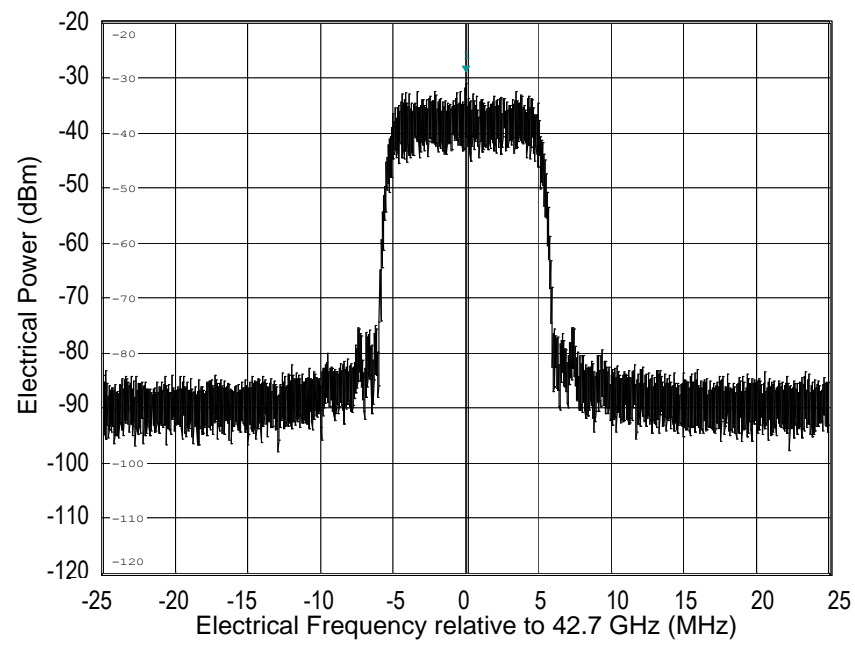

Figure 116. Radiofre quency QPSK up-converted signal spectrum at $42.7 \mathrm{GHz}$.

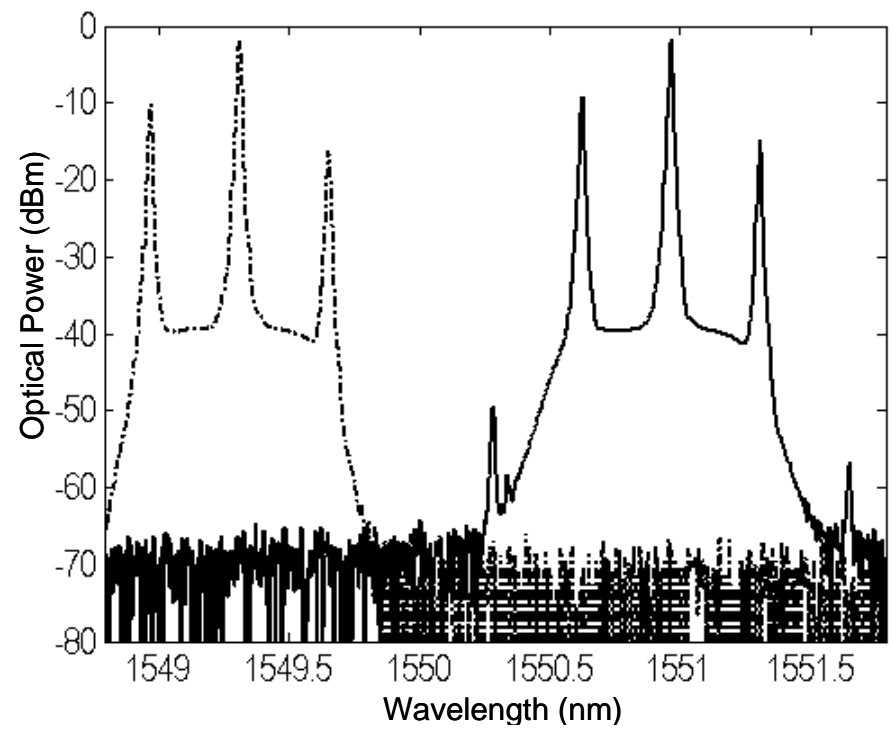

Figure 117. Optical spectra at the photodiodes input. The solid line represents the in-phase amplitude-modulated optical carrier and the das hed line corres ponds with the quadrature amplitude-modulated optical carrier.

The optical signal at the output of the second MZM is amplified in an Erbium-doped fibre amplifier (EDFA) and demultiplexed by a WDM with $0.8 \mathrm{~nm}$ of channel separation and $0.35 \mathrm{~nm}$ optical bandwidth. The optical spectrum at the two WDM outputs is depicted in Figure 117. 
Finally, both in-phase and quadrature demultiplexed optical signals are received in a photodetector (PD) with a responsivity of $\mathfrak{R}=0.7 \mathrm{~A} / \mathrm{W}$. The optical power at the PD input is $4 \mathrm{dBm}$. The received signals are electrically amplified and injected in the in-phase and quadrature inputs of a baseband digital demodulator (INTERSIL evaluation tool working in baseband mode) in order to analyze the quality of the received signals. In Figure 118 and Figure 119 the obtained constellation diagram of the received signals and the baseband spectra are respectively depicted. The measuredEVM (error vector modulator) was $12.5 \mathrm{~dB}$ and the bit energy versus noise energy (Eb/No) was $9.5 \mathrm{~dB}$. Finally, the total BER (bit error rate) obtained was $2.24 \cdot 10^{-4}$. This value was improved up to $2.7 \cdot 10^{-7}$ by using Reed Solomon forward error correction.

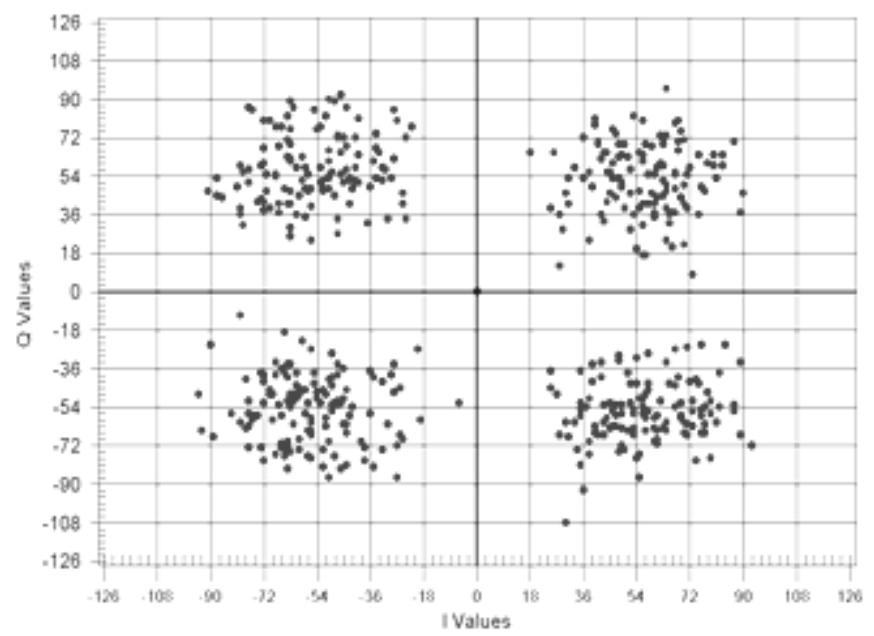

Figure 118. Demodulated constellation diagram.
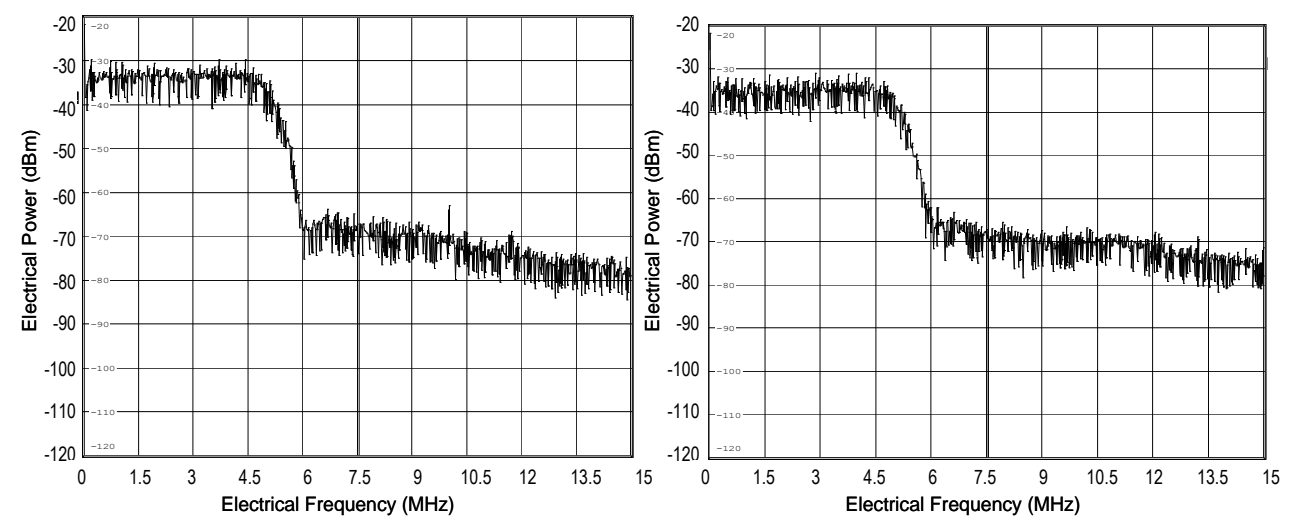

Figure 119. Received in-phase (left) and quadrature (right) spectra. 


\subsection{Conclusion}

In this chapter, a novel photonic vector demodulator architecture is described. This architecture allows the demodulation of electrical multilevel quadrature-modulated signals directly from microwave/mm-wave frequencies by using commercial photonic components and avoiding the use of bandwidth-limit ing electrical devices such as mixers and filters. The proposed technique is the first, as far as we know, where quadrature reception is done using photonic technology representing the next logical step to develop an all-optical QAM transmission/reception architecture. Measurements have demonstrated the feasibility of the proposed approach by showing the photonic reception of an $18.674 \mathrm{Mb} / \mathrm{s}$ QPSK signal at $42.7 \mathrm{GHz}$. 


\section{CHAPTER 11}

\section{Conclusion}

This thesis has been aimed at advancing the applicability of photonic technology to new areas in the field of microwave and millimeter-wave engineering through the proposal of new techniques and architectures. In particular, two main areas have been covered: optical beamforming for antenna arrays and photonic vector modulation schemes.

After an introduction of the basic building blocks in microwave photonics (chapter 1), the first five chapters have been devoted to optical beamforming. In chapter 2 a review of the main concepts has been provided including an extensive review of the state of the art. Chapter 3 reported a fibre switched TTD OBFN both in transmission and reception modes including their experimental validation and an analysis of key performance. The case of a multibeam TTD architecture was also discussed including also experiments to validate the techniques proposed. In chapter 4 , the photonic implementation of an advanced antenna functionality, multibeam capability, was studied and a new architecture was proposed and validated. The next two chapters were devoted to optical implementations of the Butler matrix in an integrated platform. In particular, chapter 5 dealt with a heterodyne optical Butler matrix architecture while chapter 6 studied a homodyne implementation.

In the second part of the thesis, photonic vector modulation of multilevel signals was studied. Different photonic-based signal processing techniques are studied to produce digital modulation (photonic vector modulation, PVM) and demodulation (PVdM). 
The practical transition to photonic implementations of microwave devices and systems is a complex paradigm shifts. During the last decades research groups all around the world have proposed a wide variety of photonic techniques and architectures that can offer advantages over microwave or digital alternatives. However, there is still further work to be done. The first stage in this progress towards a wider exploitation of the features of photonic technology is the development of new techniques and laboratory demonstrations to prove its feasibility. However, to reach the market further miniaturization and a strong reduction in cost while keeping or even enhancing its performance is needed. Photonic integration of microwave photonic solutions is therefore a key step.

This thesis has tried to contribute in these two steps with the aim of pushing microwave photonics closer to practical applications which could benefit our society. Among the different results described in this Thesis, there are two that can be considered especially relevant. The first one is the demonstration of the optical beamforming network in a chip with phase accuracy beyond the one achievable by the RF technology, which can be considered as the first example of a functional PIC component for analog applications with clear advantages for practical use. The second one is the establishment of the concept of photonic vector modulation, which was firstly studied in detail in this Thesis and resulted in the starting point to other publications and works. 


\section{References}

[ADA94] R. Adar, M. Serbin, and V. Mizrahi, "Less than $1 \mathrm{~dB}$ per meter propagation loss of silica waveguides measured using a ring resonator", J. Lightw. Technol. Vol. 12, no. 8, pp. 1369-1372, 1994.

[AGR10] G.P. Agrawal, "Fiber Optic Communication Systems", Ed. Wiley, 2010.

[AKI01] T. Akiyama, K. Inagaki, T. Ohira, and M. Hikita, "Two-dimensional optical signal processing beamformer using multilayer polymeric optical waveguide arrays," IEEE Trans. Microw. Theory Tech., vol. 49, no. 10, pp. 2055-2061, 2001.

[AKI09] T. Akiyama, H. Matsuzawa, K. Sakai, S. Itakura and Y. Hirano, "MultipleBeam Optically Controlled Beamformer Using Spatial-and-Wavelength Division Multiplexing", 2009 International Topical Meeting on Microwave Photonics, 14-16, October 2009, Valencia (Spain).

[ALA95] K. E. Alameh, R. A. Minasian, and N. Fourikis, "High Capacity Optical Interconnects for Phased Array Beamformers", Journal Of Lightwave Technology, Vol. 13, No. 6, pp. 1116-1120, June 1995.

[ALM04] V.R. Almeida, C.A. Barrios, R.R. Panepucci, M. Lipson, “All-optical control of light on a silicon chip", Nature, vol. 431, 1081-1084, 2004.

[ANA88] P. Ananasso, I. Bennion, "Integrated-Optics for on-board processing in advanced communications satellites", IEEE 1988 Global Telecommunications Conference (IEEE/GLOBECOM -88), Hollywood (PL, USA), November 28-to-December 1, 1988.

[BAU11] J.F. Bauters, M.J.R. Heck, D. John, D. Dai, M.C. Tien, J.S. Barton, A. Leinse, R.G. Heideman, D.J. Blumenthal, J.E. Bowers, "Ultra-low-loss high- 
aspect-ratio $\mathrm{Si}_{3} \mathrm{~N}_{4}$ waveguides", Optics Express, vol. 19, no. 4, pp. 3163 3174, February 2011.

[BER10] T. Berceli and P. R. Herczfeld, "Microwave photonics - a historical perspective," IEEE Transactions on Microwave Theory and Techniques, vol. 58, no. 11, pp. 2992-3000, 2010.

[BIE98] P.D. Biernacki, A. Ward, L.T. Nichols, R.D. Esman, "Microwave Phase Detection for Angle of Arrival Detection Using a 4-Channel Optical Downconverter", International Topic Meeting on Microwave Photonics (MWP98), TuB2, pp. 137-140, 1998.

[BIR92] W.S. Birkmayer, M.J. Wale, "Proof-of-concept model of a coherent optical beamforming network", IEE Proceedings-J, vol. 139, no. 4, pp. 301-304, August 1992.

[BLA04] S. Blanc, J. Lopez, L. Menager, T. Merlet, "A new coherent optical multibeam former for the receive mode in 2D antennas", Conference on Integrated optical devices, nanostructures, and displays, London, UK, 26 October 2004, vol. 5618, pp. 127-135.

[BLA60] J. Blass, "Multidirectional Antenna: A New Approach to Stacked Beams", IRE International Conference Record, Vol. 8, Part 1, 1960.

[BUR11] M. Burla, D. Marpaung, L. Zhuang, C. Roeloffzen, M. Khan, A. Leinse, M. Hoekman, and R. Heideman, "On-chip CMOS compatible reconfigurable optical delay line with separate carrier tuning for microwave photonic signal processing," Opt. Express 19, 21475-21484 (2011).

[BUT61] J.L. Butler, R. Lowe, "Beamforming Matrix Simplifies Design of Electronically Scanned Antennas”, Electronic Design, vol. 9, pp. 170-173, April 1961.

[BUT85] J.L. Butler, "Digital, Matrix, and Intermediate Frequency Scanning", Chapter 3 in Microwave Scanning Antennas, Ed. R.C. Hansen, 1985.

[CAN03] P. Candelas, J. M. Fuster, J. Martí, and J. C. Roig, "Optically generated electrical-modulation formats in digital-microwave link applications," J. Lightw. Technol., vol. 21, no. 2, pp. 496-499, Feb. 2003.

[CAP05] J. Capmany, B. Ortega, D. Pastor, and S. Sales, "Discrete-time optical processing of microwave signals," J. Lightw. Technol., vol. 23, no. 2, pp.702-723, Feb. 2005.

[CAP07] J. Capmany and D. Novak, "Microwave photonics combines two worlds," Nature Photonics, vol. 1, no. 6, pp. 319-330, 2007.

[CAP13] J. Capmany, J. Mora, I. Gasulla, J. Sancho, J. Lloret, S. Sales, "Microwave Photonic Signal Processing”, Journal of Lightwave Technology, vol. 31, no. 4, pp. 571-586, February 2013. 
[CHA90] W. Charczenko, M. Surette, P. Matthews, H. Klotz, A.R. Mickelson, "Integrated optical Butler matrix for beam forming in phased array antennas", Optoelectronics Signal Processing for Phased-Array Antennas II, pp. 196-205, 1990.

[CHA02] S. Chandramouli, W. D. Jemison, E. Funk. "Direct Carrier Modulation for Wireless Digital Comunications Using an Improved Microwave-Photonic Vector Modulator Approach", IEEE MTT-S Digest, 2002.

[CHE02] Y. Chen, and R. T. Chen, “A Fully Packaged True Time Delay Module for a K-band Phased Array Antenna System Demonstration”, IEEE Photon. Technol. Lett., Vol. 14, No. 8, August 2002, pp; 1175-1177.

[CHE08] M.Y. Chen, H. Subbaraman, R.T. Chen, "Photonic Crystal Fibre Beamformer for Multiple X -Band Phased-Array Antenna Transmissions," IEEE Photon. Technol. Lett., vol. 20, no. 5, pp. 375-377, March 1 ${ }^{\text {st }}, 2008$.

[CHO00] M. Chongcheawchamnana, K. S. Ang, D. Kpogla, S. Nam, S. Lucyszyn, I. D. Robertson "Low-cost millimetre-wave transmitter using software radio techniques", IEEE MTT-S Digest 2000, THIF-44, pp. 1949 - 1952.

[CHO03] J. Chou, Y. Han, and B. Jalali, "Adaptive RF-photonic arbitrary waveform generator,” IEEE Photon. Technol. Lett., vol. 15, no. 4, pp. 581-583, Apr. 2003.

[CHO05] C. S. Choi, H.-S. Kang, W.-Y. Choi, D.-H. Kim, and K.-S. Seo, "Phototransistors based on InP HEMTs and their applications to millimeterwave radio-on-fiber systems," IEEE Transactions on Microwave Theory and Techniques, vol. 53, pp. 256-263, January 2005

[COP99] F. Coppinger, A. Bushan, and B. Jalali, "Photonic time stretch and its application to analog-to-digital conversion," IEEE Trans. Microw. Theory Tech., vol. 47, no. 7, pp. 1309-1314, Jul. 1999.

[COR97] J.L. Corral, J. Martí, J.M. Fuster, R.I. Laming, "True time-delay scheme for feeding optically controlled phased-array antennas using chirped-fibre gratings" IEEE Photon. Technol. Lett., vol. 9, no. 11, pp. 1529-1531, November 1997.

[COR01] J.L. Corral, J. Martí, J.M. Fuster, “General expressions for IM/DD dispersive analog optical links with external modulation or optical up-conversion in a Mach-Zehnder electrooptical modulator", IEEE Transactions on Microwave Theory and Techniques (Vol.:49, Issue: 10), pp. 1968 - 1976, Oct 2001.

[COR98] J.L. Corral, J. Martí, J. Fuster and R.I. Laming, "Dispersion-induced bandwidth limitation of variable true time delay lines based on chirped fibre gratings", Electronics Letters, vol. 34, no. 2, pp. 209-211, January 1998.

[COR98b] J.L. Corral, "Application of new monolithic and photonic technologies to beamforming networks in antenna arrays for microwave and millimetre - 
wave bands", PhD. Thesis, Universidad Politécnica de Valencia, Gandia 1998.

[COR08] J.L. Corral, R. Sambaraju, M. A. Piqueras, V. Polo, "Generation of pure electrical quadrature amplitude modulation with photonic vector modulator", Optics Letters, vol. 33, no. 12, pp. 1294-1296, 2008.

[COW02] W. D. Jemison, A. J. Kreuzberger, and E. Funk, "Microwave photonic vector modulator for high-speed wireless digital communications," IEEE Microw. Wireless Compon. Lett., vol. 12, no. 4, pp. 125-127, April 2002.

[COX04] C.H. Cox, “Analog Optical Links”, Ed. Cambridge University Press, 2004.

[CUR95] D. D. Curtis, "Holographic Rotman Lens for Phased Array Antenna Beamforming", Proceedings SPIE, \#2481-17; 17 April 1995.

[DOL95] D. Dolfi, P. Joffre, J. Antoine, J.P. Huignard, D. Philippet, and P. Granger, "Experimental demonstration of a phased-array antenna optically controlled with phase and time delays", Appl. Opt., vol. 35, n² 26, September 1996.

[DUM07] P. Dumon, "Ultra-Compact Integrated Optical Filters in Silicon-on-insulator by Means of Wafer-Scale Technology", PhD, 1/3/2007, Univ. Ghent, Belgium.

[EFF10] F.J. Effenberg, J.Y. Kani, Y. Maeda, "Standardization trends and prospective views on the next generation of broadband optical access systems", IEEE Journal on Selected Areas in Communications, vol. 28, no. 6, pp. 773-780, August 2010.

[ESM93] R. D. Esman, M. Y. Frankel, J. L. Dexter, L. Goldberg, M. G. Parent, D. Stilwell, and D. G. Cooper, "Fibre-optic Prism True Time-Delay Antenna Feed”, IEEE Photon. Technol. Lett., Vol. 5, No 11, pp. 1347-1349, Nov. 1993.

[ESM95] R.D. Esman, M.Y. Frankel, M.G. Parent, "Array Transmitter/Receiver Controlled by a True Time-Delay Fibre-Optic Beamformer", IEEE Photon. Technol. Lett., vol. 7, no. 10, pp. 1216-1218, October 1995.

[ESM98] R.D. Esman, M.Y.Frankel and P. J. Mathews, "New array capabilities by photonic beamforming", IEEE MTT-S Int. Microwave Symp. Dig., Baltimore, ML, vol.3, TH2C-1, pp.1363-1366, June 1998.

[EST87] ESTEC contract $7185 / 87 / \mathrm{NL} / \mathrm{JG}(\mathrm{SC})$, on Optical Technologies for Beam Forming Networks.

[FRA95] M. Y. Frankel and R. D. Esman, "True time-delay fibre-optic control of an ultrawideband array transmitter/receiver with multibeam capability," IEEE Trans. Microw. Theory Technol., Vol. 43, No. 9, pp. 2387-2394, Sep. 1995.

[GAL97] J.T. Gallo, R. DeSalvo, "Experimental Demonstration of Optical Guided Wave Butler Matrices", IEEE Transactions on Microwave Theory and Techniques, vol. 45, no. 8, pp. 1501-1507, August 1997 
[GEN57] H. Gent, “The Bootlace Aerial,” Roy. Radar Establishment Journal, pp. 4757, October 1957.

[GHA11] N. Ghazisaidi, M. Maier, "Fiber-Wireless (FiWi) Access Networks: Challenges and Opportunities", IEEE Network, vol. 25, no. 1, pp. 36-42, January 2011.

[GOD97] L. Godara, "Applications of Antenna Arrays to Mobile Communication s, part II: Beamforming and Direction of Arrival Considerations", Proceedings of the IEEE, vol. 85, no. 8, pp. 1195-1245, August 1997.

[GOP93] G.K. Gopalakrishnan, W.K. Burns, C.H. Bulmer, "Microwave-optical mixing in LiNbO3 modulators", IEEE Transactions on Microwave Theory and Techniques, vol. 41 , no. 12, pp. 2383 - 2391, December 1993.

[GOU94] A.P. Goutzoulis, D.K. Davies, J.M. Zomp, "Development and field demostration of a hardware-compresive fibre optic true time-delay steering system of phased array antennas", Applied Optics, vol. 33, no. 35, 8173 8185, 1994.

[GRI02] R. A. Griffin and A. C. Carter, "Optical differential quadrature phase shift key (oDQPSK) for high-capacity optical transmission," Optical Fiber Communication Conference (OFC), WX6, 2002.

[GRO02] G. Grosskopf et al., "Photonic 60-GHz maximum directivity beam former for smart antennas in mobile broad-band communications," IEEE Photon. Technol. Lett., vol. 14, no. 8, pp. 1169-1171, Aug. 2002.

[HAN03] Y. Han and B. Jalali, "Photonic time-stretched analog-to-digital converter: Fundamental concepts and practical considerations," J. Lightw. Technol., vol. 21, no. 12, pp. 3085-3103, Dec. 2003.

[HEN98] S.R. Henion and P.A. Schulz, "Electrooptic Phased Array Transmitter", IEEE Photon. Technol. Lett., vol.10, n³ 3, March 1998.

[HIM98] A. Himeno, K. Kato, and T. Miya, "Silica-based planar lightwave circuits", IEEE J. Sel. Topics Quantum Electron. Vol. 4, no. 6, pp. 913 -924, 1998.

[HIR03] A. Hirata, M. Harada, and T. Nagatsuma, "120-GHz wireless link using photonic techniques for generation, modu lation, and emis sion of millime ter wave signals," J. Lightw. Technol., vol. 21, no. 10, pp. 2145-2153, Oct. 2003.

[HOC13] M. Hochberg, N.C. Harris, R. Ding, Y. Zhang, A. Novack, Z. Xuan, T. Baehr-Jones, "Silicon Photonics - The next fabless semiconductor industry", IEEE Solid-State Circuits Magazine, pp. 48-58, Winter 2013.

[HOR95] K. Horikawa, I. Ogawa, H. Ogawa, T Kitoh, "Photonic Switched True Time Delay Beam Forming Network Integrated on Silica Waveguide Circuits", in Microwave Symposium Digest, IEEE MTT-S International, Orlando, FL, USA, 16-20 May 1995, vol. 1, pp. 65-68. 
[HOR95] H. Horikawa, Y. Nakasuga and H. Ogawa, "Self heterodyning optical waveguide beam forming and steering network integrated in Lithium Niobate substrate", IEEE Trans. Microwave. Theory Tech., vol 43, n9, September 1995.

[HOW07] B. Howley, X. Wang, M. Chen, and R. T. Chen, "Reconfigurable Delay Time Polymer Planar Lightwave Circuit for an X-band Phased-Array Antenna Demonstration," Journal of Lightwave Technology, Volume: 25, Issue: 3,2007 , pp. 883-890.

[HUN09] R.G. Hunsperger, "Integrated Optics”, Ed. Springer, 2009.

[HUN99] D. Hunter and R. Minasian, "Programmable high-speed optical code recognition using fiber Bragg grating arrays," Electron. Lett., pp. 412-414, 1999.

[ISH97] T. Ishibashi, N. Shimizu, S. Kodama, H. Ito, T. Nagatsuma, and T. Furuta, "Uni-traveling-carrier photodiodes", Tech. Dig. Ultrafast Electronics and Optoelectronics (1997 OSA Spring Topical Meeting), pp. 166-168, 1997.

[JAC87] K.P. Jackson, H.P. Shaw, "Fiber-optic delay-line signal processors", Chapter 7.1, Optical Signal Processing, J.L. Horner editor, pp. 431-476, 1987.

[JEM02] W. D. Jemison, A. J. Kreuzberger, E. Funk, "Microwave Photonic Vector Modulator for High-Speed Wireless Digital Communications", IEEE Microwave and Wireless Comp. Lett., vol. 12, No. 4, April 2002.

[JIA05] Y. Jiang et.al, "Dispersion-Enhanced Photonic Crystal Fibre Array for a True Time-Delay Structured X-Band Phased Array Antenna”, IEEE Photon. Technol. Lett., Vol. 17, No.1, Jan. 2005.

[JIA05a] Z. Jia, J. Yu, and G. K. Chang, "All-optical 16 times; 2.5 Gb/s WDM signal simultaneous up-conversion based on XPM in an NOLM in ROF systems," IEEE Photonics Technology Letters, vol. 17, pp. 2724-2726, December 2005.

[JIA10] W. Jr. Jiang, C.T. Lin, C.H. Ho, C.C. Wei, P.T. Shih, J. Chen, S. Chi, "Photonic vector signal generation employing a novel optical directdetection in-phase/quadrature-phase upconversion", Journal of Lightwave Technology, vol. 35, no. 23, pp. 4069-4071, 2010.

[JOF08] L. Jofre, C. Stoltidou, S. Blanch, T. Mengual, B. Vidal, J. Martí, I. McKenzie, and J. M. del Cura, "Optically Beamformed Wideband Array Performance", IEEE Transactions on Antennas and Propagation, Vol. 56, No. 6, June 2008, pp. 1594-1604.

[JOW93] J. F. Coward, C. H. Chalfant, and P. H. Chang, "A photonic integrated optic RF phase shifter for phased array antenna beam-forming applications," J. Lightw. Technol., vol. 11, no. 12, pp. 2201-2205, December 1993. 
[JUN09] B-M. Jung et.al, "A two-dimensional optical true time-delay beamformer consisting of a fibre Bragg grating prism and switch-based fibre-optic delay lines”, IEEE Photon. Technol. Lett., Vol. 21, No. 10, 15 May, 2009.

[JUO01] P. Juodawlkis, J. Twitchell, G. Betts, J. Hargreaves, R. Younger, J. Wasserman, F. O’Donnell, K. Ray, and R. C. Williamson, "Optically sampled analog-to-digital converters," IEEE Trans. Microw. Theory Tech., vol. 49, no. 10, pp. 1840-1853, Oct. 2001.

[KAM03] V. Kaman, X. Zheng, R. J. Helkey, C. Pusarla, and J. E. Bowers, “A 32Element 8-Bit Photonic True Time-Delay System Based on a 288x288 3-D MEMS Optical Switch, IEEE Photon. Technol. Lett., vol. 15, ${ }^{\circ}$ 6, June 2003, pp.849-851.

[KIM15] B. Kim, "FTTx Migration to Giga-bps Hyper-connectivity Networking Infrastructure", Optical Fiber Conference (OFC), 2015.

[KIM99] J. K. Kim, E. Hall, O. Sjolund, and L. A. Coldren, "Room-temperature, electrically-pumped, multiple-active-region VCSELs with high differential efficiency at $1.55 \mathrm{~m}$," in Lasers and Electro-Optics Soc. Meeting 1999 (LEOS'99), Nov. 8-11, 1999.

[KIN72] R.R. Kinsey, A. L. Horvath, "Transient Response of Center-Series Fed Array,' in Phased Array Antennas, A. Oliner and G. Knittel, Artech House, pp. 261-272, 1972.

[KIS11] F. Kish, et al, "Current Status of Large-Scale InP Photonic Integrated Circuits”, IEEE J. Sel. Topics Quantum Electron. Vol. 17, no. 6, 1470-1489, 2011.

[KNI74] G.H. Knittel, "Relation of Radar Range Resolution and Signal-to-Nois e Ratio to Phased-Array Bandwidth," IEEE Trans. on Antennas and Propagation, vol. AP-22, no. 3, pp. 418-426, May 1974.

[KOE84] G.A. Koepf, "Optical processor for phased array antenna beam formation”, in Proc. SPIE, vol. 477, pp.75-81, May 1984.

[LEI99] J. Leight, J., B. Toland, "Photonic beamforming technologies for advanced military and commercial SATCOM antennas", Proceedings of 1999 IEEE Aerospace Conference, Snowmass at Aspen, USA, vol.3, pp 233-237, 6-13 March 1999.

[LEM94] L.J. Lembo, T. Holcomb, M. Wickham, P. Wisseman and J.C. Brock, "Lowloss fibre optic time-delay element for phased-array antennas", Optoelectronic Signal Processing for Phased-Array Antennas IV, SPIE vol.2155 pp. 13-23, 1994.

[LI00] B. Li, Y. Chen, Z. Fu, and R. T. Chen, "Substrate-guided wave optical true time delay feeding network for phased-array antenna steering", Proc. SPIE Optoelectronic Integrated Circuits IV, Vol. 3950, p. 256-265, April 2000. 
[LIU02] Y. Liu et al. "Wideband true time-delay unit for phased array beamforming using discrete-chirped fibre grating prism", Optics Communications 207 (2002) 177-187.

[LOA04] A. Loayssa and D. Benito, "Dispersion-tolerant all-optical subcarrier modulator for broad-band BPSK transmissions," IEEE Photon. Technol. Lett., vol. 16, no. 4, pp. 1161-1163, April 2004.

[LOA06] A. Loayssa and F. J. Lahoz, "Broad-band RF photonic phase-shifter based on stimulated Brillouin scattering and single-sideband modulation", IEEE Photon. Technol. Lett., vol. 18, no. 1, 2006.

[LOC11] D.J. Lockwoord, L. Pavesi, "Silicon Photonics”, Ed. Springer, 2011.

[MAD02] D. Madrid, B. Vidal, J.L Corral, A. Martinez, V. Polo and J. Marti, "A Novel 2N Beams Heterodyne Optical Beamforming Architecture base don NxN Optical Butler Matrices" , IEEE MTT-S International Microwave Symposium 2002, vol. 3, pp. 1945-1948, Seattle (USA), June 2-7, 2002.

[MAD10] N. Madamopoulos, "Switched Photonic Delay Lines for True Time Delay Antenna Beam Steering: Technologies and Challenges", Paper ID: NCH2010-B2, Nausivios Chora 2010.

[MAI05] R. J. Mailloux, "Phased Array Antenna Handbook", ISBN 1-58053-689-1

[MAR09] J. Martí, J. Capmany, "Microwave photonics and radio-over-fiber research", IEEE Microwave Magazine, vol. 10, no. 4, pp. 96 - 105, June 2009.

[MAR12] D. Marpaung, C. Roeloffzen, R. Heideman, A. Leinse, S. Sales, J. Capmany, "Integrated Microwave Photonics", Laser \& Photonics Reviews, November 12, 2012.

[MAT99] P. J. Matthews, "Practical photonic beamforming", Tech. Digest MWP'99, pp. 271-274, November 1999.

[MAT99] P.J. Matthews, P.L. Liu, J.B. Medberry, M.Y. Frankel, R.E. Esman, "Demonstration of a Wide-Band Fibre-Optic Nulling System for Array Antennas", IEEE Transactions on Microwave Theory and Techniques, vol. 47, no. 7, pp. 1327-1331, July 1999.

[MCP01] D. S. McPherson et al., "110 GHz Vector Modulator for Adaptive Software Controlled Transmitters", IEEE Microw. and Wireless Comp. Letters, vol. 11, no. 1, pp. 16 - 18, January 2001.

[MEN08] T. Mengual, B. Vidal, J. Martí, "Optical Phase-based Beamformer using MZM SSB Modulation combined with Crystal Polarization Optics and a Spatial Light Modulator", Optics Communications, Vol. 281, No. 2, January 15, 2008. pp. 217-224.

[MEN09] T. Mengual, B. Martínez, B. Vidal, J. Martí, "Wide-Band Nulling System for Antenna Array based on a Photonic Microwave Filter and Optical Delay 
Lines”, Optics Communications, vol. 282, no. 19, pp. 3878-3882, October 2009

[MIN09] R.A. Minasian, "Photonic Signal Processing of Microwave Signals", IEEE Transactions on Microwave Theory and Techniques, vol. 54, no. 2, pp. 832 846, February 2006.

[MIN97] R. A. Minasian, K. E. Alameh, "Optical-Fibre Grating-Based Beamforming Network for Microwave Phased Arrays," IEEE Trans. Microw.Theory Technol., vol. 45, no. 8, pp. 1513-1518, Sep. 1997.

[MIN99] R. A. Minasian and K. E. Alameh, "High capacity optical beam forming for phased arrays with fibre gratings and frequency conversion for beat noise control", Applied Optics, Vol. 38, No. 21 , pp; 4665-4670, 20 July 1999.

[MOR12] Morton, P.A.; Cardenas, J.; Khurgin, J.B.; Lipson, M., "Fast Thermal Switching of Wideband Optical Delay Line With No Long-Term Transient," IEEE Photon. Technol. Lett., vol.24, no.6, pp.512-514, March 15, 2012.

[NAG04] T. Nagatsuma, A. Hirata, M. Harada, H. Ishii, K. Machida, T. Minotani, H. Ito, T. Kosugi, and T. Shibata, "Millimeter-wave photonic integrated circuit technologies for high-speed wireless communications applications," in Proc. IEEE ISSCC, 2004, vol. 1, pp. 448-449.

[NG89] W. Ng, A. Walston, G. Tangonan, J. Newberg and J.J. Lee , "Wideband Fibre-Optic Delay Network for Phased Array Antenna Steering”, Electronics Letters, vol. 25, pp. 1456-1457, October 1989.

[NG91] W. Ng, A. Walston, G. Tangonan, J. Newberg, J.J. Lee and N. Bernstein, "The First Demonstration of an optically Steered Microwave Phased Array Antenna Using True Time Delay”, Journal ofLightwave Technology, vol. 9, pp. 1124-1131, September 1991.

[NOV98] D. Novak, G. H. Smith, C. Lim, H. F. Liu, R. B. Waterhouse, "Optically fed millimeter-wave wireless communications”, Proc. OFC '98, TuC1, pp. 14, 1998.

[OGA96] I. Ogawa, K. Horikawa, T. Kitoh and H. Ogawa, "Novel multibeam forming network miniaturized by optical slab waveguide“, Proc. ISAP 96, Japan, pp 121-124.

[OHA03] K. Ohata, K. Maruhasi, M. Ito, S. Kishimoto, K. Ikuina, T. Hashiguchi, K. Ikeda, and N. Takahashi, " $1.25 \mathrm{~Gb} / \mathrm{s}$ wireless gigabit Ethernet link at 60 GHz-band,” in Proc. IEEE Int. Microw. Symp., 2003, pp. 373-376.

[ORT00] B. Ortega, J.L. Cruz, J. Capmany, M.V. Andrés, D. Pastor, "Variable delay line for phased array antennas based on a chirped fibre grating", IEEE Transactions on Microwave Theory and Techniques, vol.48, no. 8, pp. 1352 1360, August 2000. 
[PAU99] D. K. Paul, "Photonic beamforming and steering for SATCOM phased arrays", WFFT Workshop notes, 1999 IEEE MTT-S International Microwave Symposium (1999).

[PIQ06] M. A. Piqueras et al., "Optically beamformed beam switched adaptive antennas for fixed and mobile broadband wireless access networks," IEEE Trans. Microwave Theory Tech., vol. 54, no. 2, pp. 887-899, Feb. 2006.

[PIQ06a] M. Á. Piqueras, B. Vidal, H. Pfrommer, V. Polo, A. Ramírez, J. Martí, "Radio-over-fiber multi-service MM-wave interconnection with photonic up-conversion, dual band remote delivery and photonic envelop detection," in Proc. IEEE MTT-S, Intl. Microwave Symp., San Francisco, CA, Jun. 2006, pp. 2035-2038.

[PIQ06b] M. A. Piqueras et al., "Radio-Over-Fibre Broadband Quadrature Amplitude Modulator", 32nd European Conference on Optical Communications (ECOC), Cannes (France), September 24-28, 2006.

[RAD03] A. Rader and B.L. Anders on, "Demonstration of a linear true time delay device by use of a microelectromechanical mirror array," Applied Optics, vol. 42, no. 8, p. 1409, 2003.

[RAZ05] O. Raz, R. Rotman, and M. Tur, "Wavelength-Controlled Photonic True Time Delay for Wide-Band Applications", IEEE Photon. Technol. Lett., Vol. 17, NO. 5, May 2005, pp. 1076-1078.

[RIB95] R.F.S. Ribeiro, J.R.F. da Rocha, A.V.T. Cartaxo, "Influence of Laser Phase Noise on Dispersive Fibre Communication Systems", IEEE Photon. Technol. Lett., vol. 7, no. 12, pp. 1510-1512, December 1995.

[RIZ92] N. Riza, "Liquid crystal-based optical control of phased-array antennas", Journal of Lightwwave Technology, vol. 10, no. 12, pp. 1974-1984, December 1992.

[ROT63] W. Rotman, R.F. Turner, "Wide Angle Microwave Lens for Line Source Applications", IEEE Trans. on Antennas and Propagation, vol. AP-11, pp. 623-632, 1963.

[ROU98] H. Roussell and R. Helkey, "Optical frequency conversion using a linearized LiNbO3 modulator," IEEE Microw. Guided Wave Lett., vol. 8, no. 11, pp. 408-410, Nov. 1998.

[SAM07] R. Sambaraju, M. Á. Piqueras, V. Polo, J. L. Corral, J. Martí, "Generation of Multi-Gigabit-per-Second MQAM/MPSK-Modulated Millimeter-W ave Carriers Employing Photonic Vector Modulator Techniques", Journal of Lightwave Technology, vol. 25, no. 1, pp.3350-3357, 2007.

[SAM08] R. Sambaraju, J.L. Corral, J. Palací, V. Polo, J. Martí, "Performance Analysis of Photonic Vector Modulation Techniques for Multi-Gb/s Wireless Links", Journal of Lightwave Technology, vol. 2, no. 15, pp. 26842691, 2008. 
[SAN06] P. Sanchis, et al. "WP4.1 Report - Breadbord fabrication (Part I)", European Space Agency under the Innovative Triangle Initiative study "Optical beamforming network for multibeam satellite-on-board phased-array antennas" (19785/06/NL/PA).

[SEE02] A. J. Seeds, "Microwave photonics," IEEE Transactions on Microwave Theory and Techniques, vol. 50, no. 3, pp. 877-887, 2002.

[SEE93] A.J. Seeds, "Optical technologies for phased array antennas”, IEICE Trans. on Electronics, vol. E76-C, pp. 198-206, 1993.

[SMI97] G. H. Smith, D. Novak, Z. Ahmed, "Technique for optical SSB generation to overcome dispersion penalties in fibre-radio systems", Electron. Lett., vol. 33, no. 1, pp. 74-75, 1997.

[SOR84] R. A. Soref, "Programmable time-delay devices", Applied Optics, vol. 23, no. 21, pp. 3736-3737, Nov. 1984.

[SOR92] R. Soref, “Optical Dispersion Technique for Time-Delay Beam Steering”, Applied Optics, vol. 31, pp. 7395-7397, December 10, 1992.

[SPA00] R. A. Sparks, "Progress in optical Rotman beamformer technology", Proceedings of the IEEE International Conference on Phased Array Systems and Technology, pp. 357-360, 2000.

[SPA98] R. A. Sparks, et al., "Experimental Demonstration of a Fibre Optic Rotman Beamformer", Tech. Digest MWP '98, pp. 127-1, 30 October 1998.

[SPA99] R. A. Sparks, N. Slawsby, "Eight beam prototype fibre optic Rotman lens", Tech. Digest MWP '99, pp. 283-286, 19 November 1999.

[STU00] J. Stulemeijer, R. van Dijk, F. E. van Vliet, D. H. P. Maat, and M. K. Smit, "Photonics chip for steering a four element phased array antenna," in Proc. IEEE/IEE MWP, Oxford, UK. 2000, pp. 20-22.

[STU99] J. Stulemeijer, F. van Vliet, K. Benoist, D. Maat, M. Smit, "Compact photonic integrated phase and amplitude controller for phased-array antennas", IEEE Photon. Technol. Lett., vol 11, no. 1, pp. 122 -124, 1999.

[SUR93] Surette, M.R., Hjelme, D.R., Mickelson, A.R., "An optically driven Phased Array antenna utilizing Heterodyne Techniques", Journal of Lightwave Technology Vol 11 (9) pp. 1500-1509 September 1993

[TAY79] H.F. Taylor, "Fiber and integrated optical devices for signal processing", SPIE, vol. 176, pp. 17-27, 1979.

[TED95] S. Tedjini, A. Ho-Quoc, D.M. Khalil, "All-optical networks as microwave and millimetre-wave circuits", IEEE Transactions on Microwave Theory and Techniques, vol. 43, no. 9, pp. 2428-2434, September 1995.

[THA90] S. Thaniyavarn, G.L. Abbas, W.A. Dougherty, "Milimeter-wave signal generation and control using optical heterodyne techniques and electrooptics devices”, High-frequency Analog Fibre Optic Systems, 1990. 
[THO15] V. Thomas, M. El-Hajjar, and L. Hanzo, "Performance improvement and cost reduction techniques for radio over fiber communications," IEEE Communications Surveys \& Tutorials, vol. PP, no. 99, pp. 1-1, 2015

[THO15a] V. A. Thomas, M. El-Hajjar, L. Hanzo, "Millimeter-Wave Radio Over Fiber Optical Upconversion Techniques relying on Link Non-Linearity", IEEE Communications Surveys \& Tutorials, 2015.

[THY14] L. Thylén, L. Wosinski, “Integrated photonics in the $21^{\text {st }}$ century”, Photonics Research, vol. 2, no. 2, pp. 75-81, April 2014.

[TN2] M. A. Piqueras, et al. "Technical Note $n^{\circ} 2$ - Principle of Operation of the Optical Beamforming Network based on Integrated Optical Butler Matrixes . Heterodyne generation and 2N beam extension", European Space Agency under the Innovative Triangle Initiative study "Optical beamforming network for multibeam satellite-on-board phased-array antennas" (19785/06/NL/PA).

[TN3] G. Caille, "Technical Note $n^{\circ} 3$-Upgrade of the antenna BFN architecture: interest to use the 8 central beams among 16 close ones from an innovative optical 8x8 matrix UPV design.”, European Space Agency under the Innovative Triangle Initiative study "Optical beamforming network for multibeam satellite-on-board phased-array antennas" (19785/06/NL/PA).

[TN4] G. Caille, "Technical Note TN4 - Effect of errors measured on the $8 \times 8$ Butler matrix for a full-size antenna", European Space Agency under the Innovative Triangle Initiative study "Optical beamforming network for multibeam satellite-on-board phased-array antennas" (19785/06/NL/PA).

[TON06] S. Tonda-Goldstein, D. Dolfi, A. Monsterleet, S. Formont, J. Chazelas, J. P. Huignard, "Optical Signal Processing in Radar Systems", IEEE Transactions on Microwave Theory and Techniques, vol. 54, no. 2, pp.847-853, February 2006.

[TON96] D. T. K. Tong, M. C. Wu, "A novel multiwavelength optically controlled phased array antenna with a programmable dispersion matrix," IEEE Photon. Technol. Lett. 8, 812-814, 1996.

[TON96b] D.K.T. Tong, M.C. Wu, "Programmable dispersion matrix using Bragg fibre grating for optically controlled phased array antennas", Electron. Lett., vol. 32, no, 17, 1532-1533, 1996.

[TOR11] A. Torre, P. Capece, "COSMO-SkyMed: the advanced SAR instrument", 5 International Conference on Recent Advances in Space Technologies (RAST), pp. 865-868, June 2011.

[TUR09] M. Tur, "True time delay photonic beamforming: A review", IEEE International Conference on Microwaves, Communications, Antennas and Electronics Systems (COMCAS 2009), Tel Aviv, June 2009. 
[URI15] V.J. Urick, K.J. Williams, J.D. McKinney, "Fundamentals of Microwave Photonics", Ed. Wiley, March 2015.

[VID02] B. Vidal, J.L. Corral, M.A. Piqueras, J. Martí, "Optical Delay Line Based on Arrayed Waveguide Gratings' Spectral Periodicity and Dispersive Media for Antenna Beamforming Applications", IEEE J. Selected Topics Quantum Electron., vol. 8, 1202-1210, 2002.

[VID02] B. Vidal, D. Madrid, J.L. Corral, J. Martí, "Novel Photonic True Time Delay Beamformer based on the Free Spectral Range Periodicity of Arrayed Waveguide Gratings and Fibre Dispersion", IEEE Photonic Technology Letters, vol. 14, no. 11, pp. 1614-1616, November 2002.

[VID03] B. Vidal, V. Polo, J. L. Corral, J. Martí, "Multibeam Optical Beamforming Architectures for Broadband Wireless Access Networks", IST Mobile \& Wireless Telecommunications Summit 2003, Aveiro (Portugal), 15-18 June, 2003.

[VID03b] B. Vidal, V. Polo, J. L. Corral, J. Martí, "Photonic microwave filter with tuning and reconfiguration capabilities using optical switches and dispersive media”, Electronics Letters, vol. 39, no. 6, pp. 547-549, 20th March 2003.

[VID04] B. Vidal, "Aplicaciones de líneas de retardo WDM al procesado óptico de señales eléctricas", PhD. Thesis, Universidad Politécnica de Valencia, Valencia, 2004.

[VID06] B. Vidal, T. Mengual, C. Ibáñez-López, and J. Marti, “Optical Beamforming Network Based on Fibre-Optical Delay Lines and Spatial Light Modulators for Large Antenna Arrays", IEEE Photon. Technol. Lett., Vol. 18, No. 24, December15, 2006, pp.2590-2592.

[VID12] B. Vidal, T. Nagatsuma, N.J. Gomes, T.E. Darcie, "Photonic Technologies for Millimeter- and Submillimeter-wave Signals", Advances in Optical Technology, vol. 2012, September 2012.

[VID12] B. Vidal, T. Mengual, J. Martí, "Fast Optical Beamforming Architectures for Satellite-based Applications", Advances in Optical Technology, vol. 2012, 385409, September 2012.

[VLI99] F.E. van Vliet, J. Stulemeijer, K.W. Benoist, D.P.H. Maat, M.K. Smit, \& R. van Dijk, "Photonic Integrated Circuits for Phased-Array Beamforming"; Proceedings of the Conference Perspectives on Radio Astronomy: Technologies for Large Antenna Arrays, ASTRON Institute, Dwingeloo, 12 14 April 1999, p.295.

[VOD03] N. Vodjdani, G. Granger, D. Mongardien, A. Enard, C. Fourdin, J. Chazelas, "5 bits wideband optical beam steering up to Ku band", MWP Workshop, pp. 389-391, 10-12 September, 2003. 
[WAK10] D. Wake, A. Nkansah, and N. Gomes, "Radio over fiber link design for next generation wireless systems," IEEE/OSA Journal of Lightwave Technology, vol. 28, pp. 2456 -2464, August 2010.

[WAN95] J. Wang, K. Petermann, K., "Small Signal Analysis for Dispersive Optical Fibre Communication Systems", Journal of Lightwave Technology, vol. 10, no. 1, pp. 96-100, January 1992.

[WIB05] A. Wiberg, P. Perez-Milan, M. V. Andres, P. A. Andreks on, P. O. Hedekvist, "Fiber-optic 40-GHz mm-wave link with $2.5-\mathrm{Gb} / \mathrm{s}$ data transmission," IEEE Photon. Technol. Lett., vol. 17, no. 9, pp. 1938-1940, Sep. 2005.

[WIL08] R. C. Williamson and R. D. Esman, "RF photonics," Journal of Lightwave Technology, vol. 26, no. 9, pp. 1145-1153, 2008

[WIL15] K.A. Williams, E.A.J.M. Bente, D. Heiss, Y. Jiao, K. Lawniczuk, X.J.M. Leijtens, J.J.G.M. van der Tol, M.K. Smit, "InP photonic circuits using generic integration", Photonics Research, vol. 3, no. 5, pp. B60-B68, October 2015.

[WIL76] K. Wilner, A.P. Van Den Heuvel, "Fiber Optic Delay Lines for Microwave Signal Processing”, Proceedings of the IEEE, vol. 64, pp. 805-807, 1976.

[www1] www.light2015.org

[www2] www.epixfab.eu

[XU05] Q. Xu, B. Schmidt, S. Pradhan, M. Lipson, "Micrometre-scale silicon electro-optic modulator", Nature, vol. 435, 325-327, 2005.

[YAM90] S. Yamamoto, N. Edagawa, H. Taga, Y. Yoshida, H. Wakabayashi, "Analysis of Laser Phase Noise to Intensity Noise Conversion by Chromatic Dispersion in Intensity Modulation and Direct Detection Optical-Fibre Transmission", Journal of Lightwave Technology, vol. 8, no. 11, pp. 17161722, November 1990.

[YAO98] X.S. Yao, "Phase-to-amplitude modulation conversion using Brillouin selective sideband amplification." IEEE Photon. Technol. Lett., vol. 10, no. 2, pp 264-266, February 1998.

[YAO09] J. Yao, "Microwave photonics," Journal of Lightwave Technology, vol. 27, no. 3, pp. 314-335, 2009.

[YAO15] J. Yao, "Photonics to the Rescue", IEEE Microwave Magazine, pp. 46-60, September 2015

[YAR10] Yaron, L.; Rotman, R.; Zach, S.; Tur, M., "Photonic Beamformer Receiver With Multiple Beam Capabilities." IEEE Photon. Technol. Lett., vol.22, no.23, pp. 1723-1725, Dec.1, 2010.

[YEG00] S. Yegnanarayanan, and B. Jalali, "Wavelength-Selective True Time Delay for Optical Control of Phased-Array Antenna", IEEE Photon. Technol. Lett., vol. 12, no. 8, pp. 1049-1051, August 2000. 
[YEG96] S. Yegnanarayanan, P.D. Trinh and B. Jalali, "Recirculating photonic filter: a wavelength selective time delay for phased-array antennas and wavelength code-division multiple access", Optics Letters, vol. 21, no. 10, pp. 740-742, 15th May 1996.

[YIF05] L. Yifei, M. Bystrom, D. Yoo, S.M. Goldwasser, P.R. Herczfeld, "Coherent optical vector modulation for fiber radio using electrooptic microchip lasers", IEEE Transactions on Microwave Theory and Techniques, vol. 53, no. 10 , pp. $3121-3129$, Oct. 2005.

[ZAL09] Z. Zalevsky, S. Zach and M Tur, "A Novel Photonic Rotman-Lens Design for Radar Phased Array", IEEE International Conference on Microwaves, Communications, Antennas and Electronics Systems 2009, COMCAS 2009, Tel Aviv, Israel, , 9-11 Nov. 2009, pp. 1-4.

[ZHA98] W. Zhang, J.A.R. Williams, L.A. Everall, I. Bennion, "Fibre optic radio frequency notch filter with linear and continuous tuning by using a chirped fibre grating”, IEE Electronics Letters, vol. 34, no. 18, pp. 1770-1772, 3rd September 1998.

[ZHA08] Y. Zhang, K. Xu, R. Zhu, J. Li, J. Wu, X. Hong, J. Lin, "Photonic Generation of M-QAM/M-ASK Signals at Microwave/Millimeter-Wave Band Using Dual-Drive Mach-Zehnder Modulators With Unequal Amplitudes”, Journal of Lightwave Technology, vol. 26, no. 15, pp. 2604-2610, 2008.

[ZHU06] L. Zhuang, C. G. H. Roeloffzen, R. G. Heideman, A. Borreman, A. Meijerink, W. van Etten,"Ring resonator-based single-chip $1 \times 8$ optical beam forming network in LPCVD waveguide technology," Proc. 11th IEEE/LEOS Symp. Benelux, Eindhoven, The Netherlands, 30 Nov.-1 Dec. 2006, pp. 45-48.

[ZMU94] H. Zmuda, E.N. Toughlian, Photonic Aspects of Modern Radar, Ed. Artech House, London, 1994. 


\section{Annex A: Impact of element failures in an Optical Butler Matrix}

In this Annex, the effect on the radiation pattern of failures in array elements due to degradations in the optical Butler Matrix (BM) is discussed.

\section{A.1 Basic formulas for the effect of errors in large antenna arrays}

Until this point it has been assumed that the implementation of the OBFN and the antenna array was a perfect copy of the design. However, in a practical situation, errors may arise affecting the radiation performance of the system. BFN errors are modeled by considering that, for each beam, antenna complex excitation coefficients $\underline{a}_{n}$ (See equation 2.1) become stochastic variables with a Gaussian distribution with small variance. The latter is assumed to be the same for all paths, as the concerned BFN architecture is quite symmetrical with respect to all of them. If we assume that no element fails, it implies that the mean of the amplitude and the phase errors are equal to 0 . The effect of amplitude and phase errors caused by the imperfect beamforming network on the directivity and residual sidelobe level of the radiation pattern are given by [Cor98]: 


$$
\begin{gathered}
\overline{\sigma^{2}} \approx \frac{\left(1+\overline{\Delta^{2}}\right) e^{\overline{\delta^{2}}}-P}{\eta N P} \approx \frac{\left(1+\overline{\Delta^{2}}\right)\left(1+\overline{\delta^{2}}\right)-P}{\eta N P} \approx \frac{1+\overline{\Delta^{2}}+\overline{\delta^{2}}-P}{\eta N P} \\
\frac{D}{D_{0}}=\frac{P}{\left(1+\overline{\Delta^{2}}\right) e^{-\overline{\delta^{2}}}} \approx \frac{P}{\left(1+\overline{\Delta^{2}}\right)\left(1+\overline{\delta^{2}}\right)} \approx \frac{P}{1+\overline{\Delta^{2}}+\overline{\delta^{2}}}
\end{gathered}
$$

where $\overline{\sigma^{2}}$ is the residual sidelobe level, $\overline{\Delta^{2}}$ and $\overline{\delta^{2}}$ are the variances of amplitude and phase errors and $\eta$ is the illumination efficiency (that equals 1 for an uniform amplitude distribution). $P$ is the probability that any path will remain well-operating at the end of a given mission-life; i.e. (1- $P$ ) is the probability of failure, or practically in a large array the number of failed paths. The BM being a passive optical network is considered without failure. So, without failure $(P=1)$, using decibels unity, keeping only the $1^{\text {st }}$ term of the Taylor development of the decimal log function for low error variance values, and taking into account that the illumination efficiency $\eta=1$ for uniform amplitude the previous formulas are simplified as follows:

$$
\text { Directivity decrease: } \quad \Delta D_{d B}=-4.34\left(\overline{\Delta^{2}}+\overline{\delta^{2}}\right)
$$

Spurious Level (average of random sidelobes): $S L_{d B / \max }=\left(\overline{\Delta^{2}}+\overline{\delta^{2}}\right) / N$

The complex coefficients, $\underline{a}_{n}{ }^{15}$,can be defined by their module with average value equal to 1 (the array without error has equal amplitude excitations), and their phase ${ }^{16}$. $\sigma_{\varphi}$ is the common standard deviation applicable to all $\arg \left(a_{n}\right)$, each around its specific mean value. Note that when combining various kinds of cascaded phase errors, they should be "RSS summed":

$$
\delta^{2}=\sum \sigma_{\varphi}^{2}
$$

The amplitude errors are characterized by a standard deviation, $\Delta$, that follows a Rayleigh probability law [MAI05].

Using decibel units is more convenient, as usually this parameter is directly obtained from the measurements: $\mathrm{A}_{\mathrm{n}}(\mathrm{dB})=20 \log _{10}\left[\operatorname{mag}\left(\underline{a}_{n}\right)\right]$. By using the $1^{\text {st }}$

${ }^{15}$ In this Annex, complex quantities are mentioned with underlined letters. A letter with a line above, such as $\bar{\delta}$, means that the average value of the concerned variable (here $\delta$ ) is taken.

16 The above formulas use 'radians' unit; so a translation should be used from the measured values in degrees (with standard deviation $\sigma^{\circ}$ ): $\sigma_{\varphi}=\pi / 180 \cdot \sigma^{\circ}$ 
term of the Taylor expansion of the 'decimal log' function, $\Delta$ can be linked for rather small errors to the standard deviation $\sigma$ measured in $\mathrm{dB}$ as:

$$
\sigma(\mathrm{dB})=8.68 \sigma_{\mathrm{a}}
$$

To apply these basic formulas to the optical beamforming network described in Chapters 5 and 6, the next considerations should be taken into account:

$\circ \overline{\Delta^{2}}$ and $\overline{\delta^{2}}$ should take into account errors occurring at the various levels of the BFN. According to Figure 65, the BFN comprises 2 main cascaded parts: the 'horizontal' $8 \rightarrow 16$ matrices, and the 'vertical' $6 \rightarrow 16$. Each part has a row of $3 \mathrm{~dB}$ couplers, then a cascaded $8 \times 8$ Butler Matrix ( 2 side by side).

- Errors come mainly from manufacturing uncertainties, so they are modeled as uncorrelated at all levels, especially between the $1^{\text {st }}$ and $2^{\text {nd }}$ stages; so the variances coming from the various stages are added ('RSS' summation) within $\overline{\Delta^{2}}$ and $\overline{\delta^{2}}$. The final $\mathrm{A} / \varphi^{17}$ value at each radiating element level is the sum in $\mathrm{dB}$ and phases of the transfer functions (or S-matrix transmission coefficients), cascaded for the 4 successive stages:

1) $1^{\text {st }}$ coupler row at input of the "horizontal slides".

2) $1^{\text {st }}, 8 \times 8$ optical Butler Matrix and then in the "vertical slides".

3) $2^{\text {nd }}$ coupler row.

4) $2^{\text {nd }}$ set of $8 \times 8$ Butler Matrices.

- The RSS summing applies both to phase errors (see equation A.5), and to amplitude errors according to:

$$
\Delta^{2}=\sum \sigma_{a}^{2}
$$

Both categories (phase \& amplitude errors) are assumed to be uncorrelated. Finally, it is important to note that:

- No error dispersion of the input $1 \rightarrow 8$ switches has to be taken into account because only one position of each switch acts for a given spot-beam.

- For the same reason, errors coming from using the " $\mathrm{N} \rightarrow 2 \mathrm{~N}$ " switch can be neglected, because its main effect is to change the selected beam.

- However further more detailed analyses at the whole RF/optical beamforming subsystem level (a priori and as a retrofit from measurements) should take into account dispersions among the various 'input demux' (alias "sideband extractors"), reference splitter and output mixers (gathering again sidebands and the spectrum main-lobe). It is assumed than the latter unbalances will be low with respect to those accruing within the optical BFN itself (Butler matrices and associated front couplers rows).

${ }^{17} \underline{\mathrm{A} / \varphi}$ : amplitude \& phase. 


\section{Annex B: Fabrication process in SOI}

In this Annex, the fabrication process employed for the implementation of the optical Butler matrices described in Chapters 5 and 6 and carried out in the Nanophotonics Technology Center is briefly described as well as the analysis of the effect of the waveguide roughness in the phase performance of the Butler Matrix.

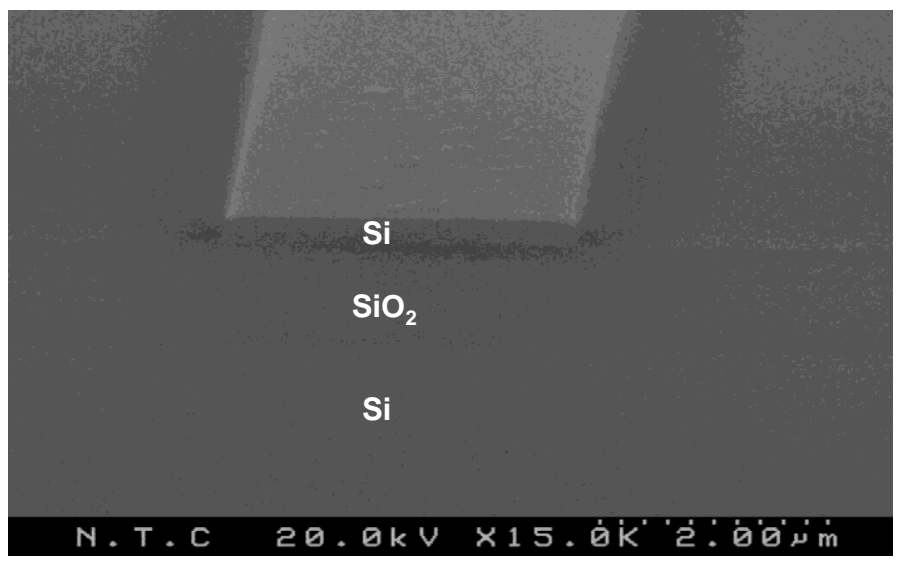

Figure B.1. SEM image of a SOI wave guide. It can be clearly seen the top silicon layer, the oxide cladding layer (B OX layer) and the silicon substrate . 
First of all, it must be pointed out that the employed technology for the fabrication of the different components was Silicon-on-insulator (SOI). Silicon-on-insulator (SOI) consists of a thin silicon layer on top of an oxide cladding layer deposited on a bare silicon substrate. The used SOI wafers are made of a top silicon layer of a thickness of $200 \mathrm{~nm}$ and an underlying silica layer of a thickness of $3 \mu \mathrm{m}$. Figure B.1 shows a scanning electronic microscope (SEM) image of a SOI waveguide. Figure B. 2 summarizes the fabrication processes.

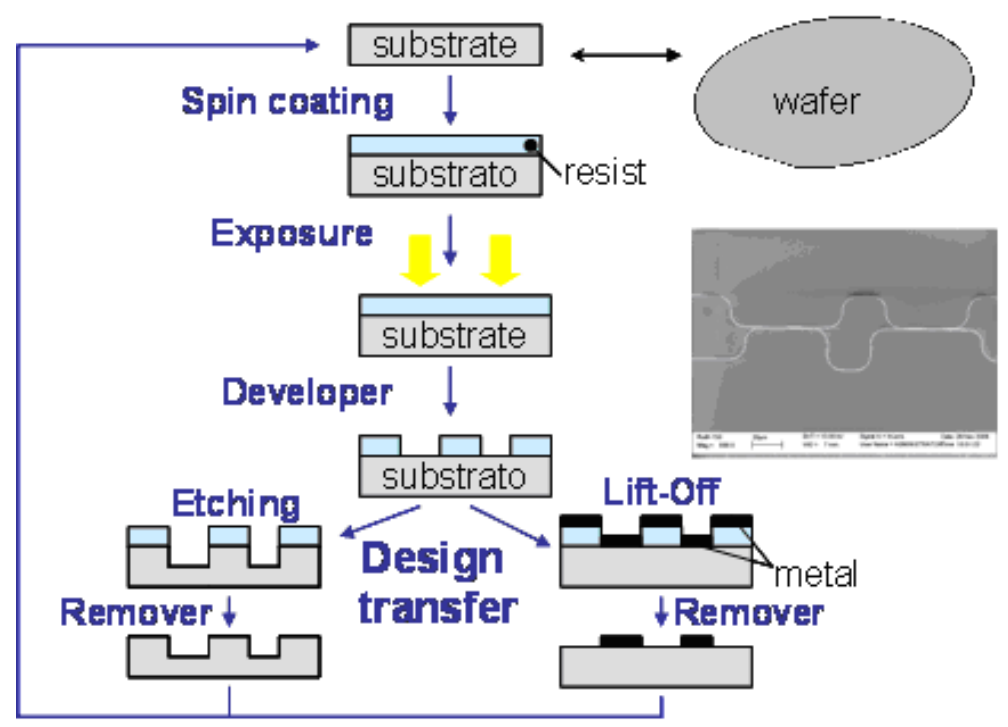

Figure B.2. Schematic of the fabrication processes. A fabricated Mach-Zehnder Interfe rometer using the lift-off process, which includes two optical couplers and two phase shifters, is depicted in the inset.

As shown in Figure B.2, two different processes have been used depending on the mask material for etching purposes. In the first case, the resist is used as mask while in the second case (lift-off process) a metal is employed to achieve the etching mask. As it will be shown later, better result are obtained by using lift-off process, in terms of slope and ripple of the sidewalls of the structures.

There are several common steps, before etching, that are now briefly described:

\section{Spin Coating}

A typical coater (EVG-101) was employed for the deposition of the resist over the SOI wafer or sample. PMMA resist was the selected resist for all cases.

\section{Exposure}


Among different methods, e-beam lithography was selected for exposure. A Raith150 system was employed for this purpose achieving a high resolution.

It must be pointed out that, depending on the etching process, the exposure pattern for the same structure and resist (PMMA) will be different as we can see in Figure B.3.

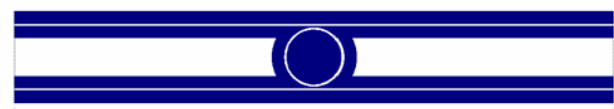

(a)

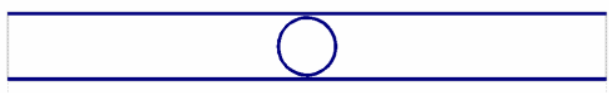

(b)

\section{Figure B.3. Exposure patterns for (a) resist mask or (b) lift-off process.}

In the first case, when the resist is used as a mask, the trenches of the design are exposed while for lift-off process the pattern is directly exposed. As mentioned above, the lift-off process was finally employed, so the layouts for the different building blocks were drawn as depicted in Figure B.3(b). Figure B.4 shows the layout for the building block consisting of different crosses.

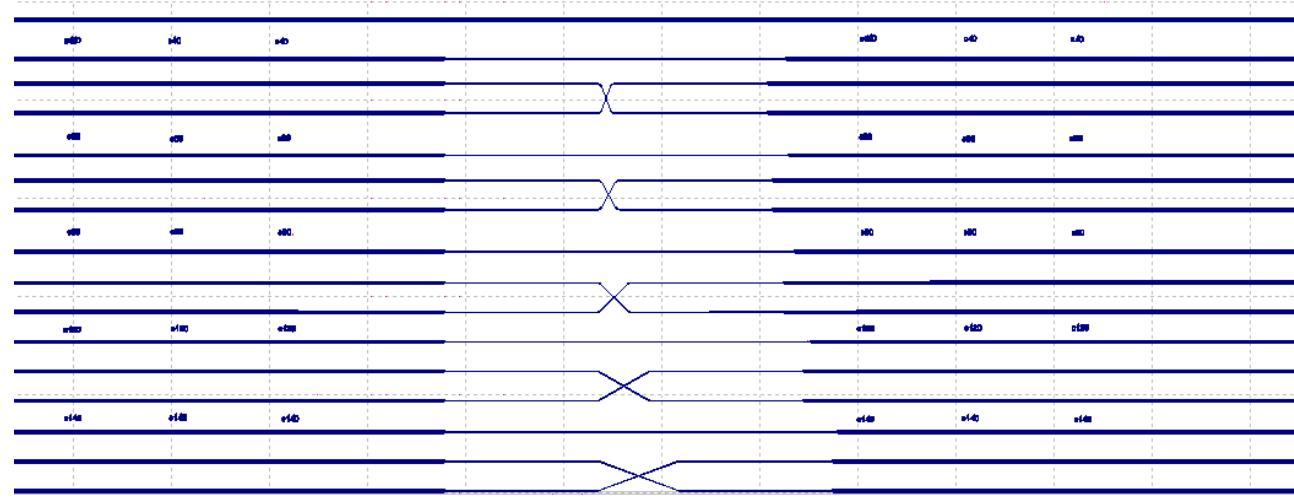

\section{Figure B.4. Lay-out for building block consisting on diffe re nt angles for wave guide crosses.}

It must be pointed out that the lithography strategy is a key factor to achieve a good transfer of the lay-out to the physical sample. Different parameters of the lithography process have been adjusted and checked by NTC fabrication team through the development of several test samples. So, the dose of the different elements has been studied and adjusted as well as other parameters as the waiting time of the beam or the way of decomposing the patterns (polygons or line) when exposing. Figures B.5 and B.6 show how the fine adjustment of all those parameters 
can improve the final result for the coupler separation resolution or the sidewall roughness, for instance.

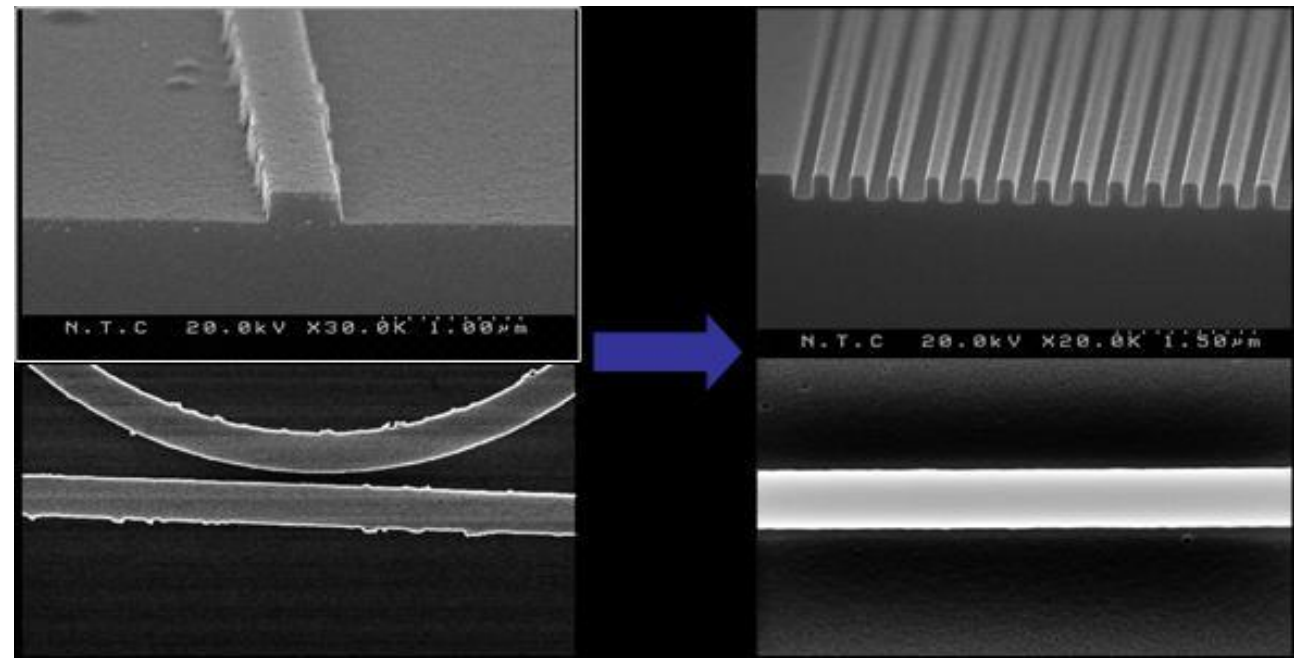

Figure B.5. Improved sidewall roughness by modifying e -beam lithography process and tuning the bias during the etch step.
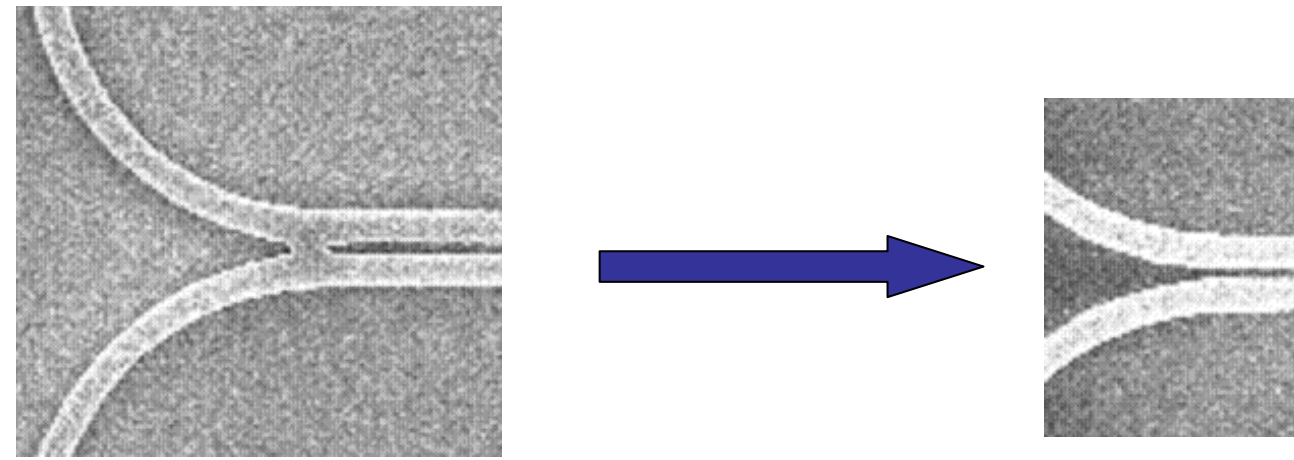

Figure B.6. Improved results after exposure dose adjustments.

\section{Developing}

The developing process is used to transfer the designed pattern to the resist. In our case, the developer system employed was the TEL REVE 20 Hot Plate that allows the developing of the samples as well as the realization of different bake procedures.

\section{Etching process}


As previously mentioned, two different approaches were considered to create the mask for the substrate etching. In the first case, the resist is directly employed as mask while in the second case, and after a lift-off process, a chromium layer is deposited for acting as the etching mask. The chromium was deposited by using an evaporation process (PFEIFFER Vacuum classic 500) that ensures a good distribution of the metal over the resist.

It should be pointed out that the lift--ff process ensures better results in terms of sidewalls roughness of the guides. This roughness reduction is a key factor for controlling the phase of the propagating light signal through the guide. Figure B.7 shows a comparison between a final guide obtained by resist mask (a) and a guide achieved by lift-off process (b).
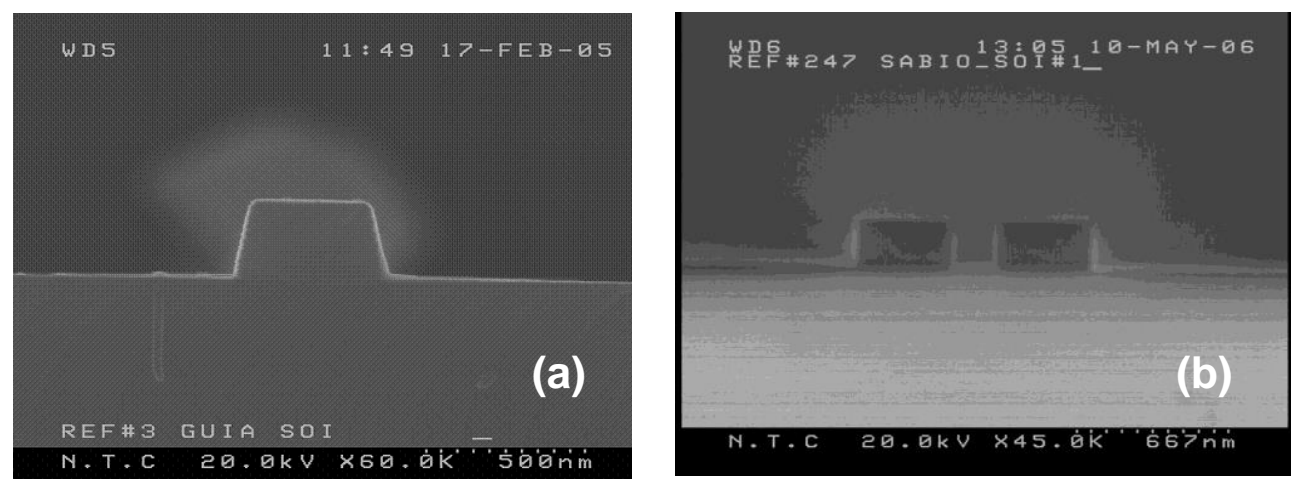

Figure B.7. Guide roughness comparis on between resist mas k etching (a) and lift-off process (b).

The etching process was carried out by using a RIE-ICP (Reactive Ion Etching with Inductive Plasma Source) that has different gases for etching Silicon as well as Silica. The etching process and the interaction between developing and etching methods were also optimized by NTC fabrication team, achieving better final results in terms of pattern transferring and propagation losses reduction.

Till the building block fabrication, PMMA resist was used as medium to transfer the patterns in the e-beam procedure. Two different approaches were employed with PMMA: direct photolithography and lift-off procedure. But both approaches resulted limited by the PMMA resolution: $20 \mathrm{~nm}$. In order to improve pattern transfer resolution as well as the roughness of the final devices, a new process based on the use of a higher resolution resist was developed for the matrix fabrication.

The selected resist was a negative resist, i.e. the exposed area remains after e-beam exposure. The use of such resist gets a better resolution and lower roughness what is translated to lower propagation losses. Propagation losses lower than 
$10 \mathrm{~dB} / \mathrm{cm}$ at the operating wavelength of $1550 \mathrm{~nm}$ has been achieved with this new process thus improving the $15 \mathrm{~dB} / \mathrm{cm}$ obtained by using the previous process. The employed scenario for fabrication by using the negative resist is depicted in the following Figure B.8.

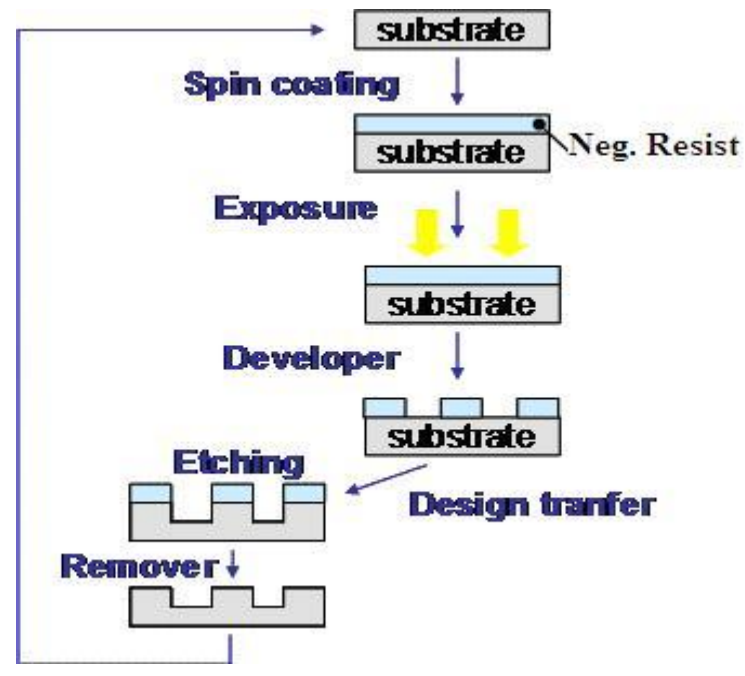

Figure B.8. Schematic of the fabrication process.

Figure B.9 shows two SEM images of a coupler defined (a) with a standard process and (b) including proximity effect compensation strategies. It can be seen how the use of proximity effect compensation improves the roughness of the waveguides thus decreasing the propagation losses, improving the behaviour of the different devices and reducing the width deviations in the fabrication process.

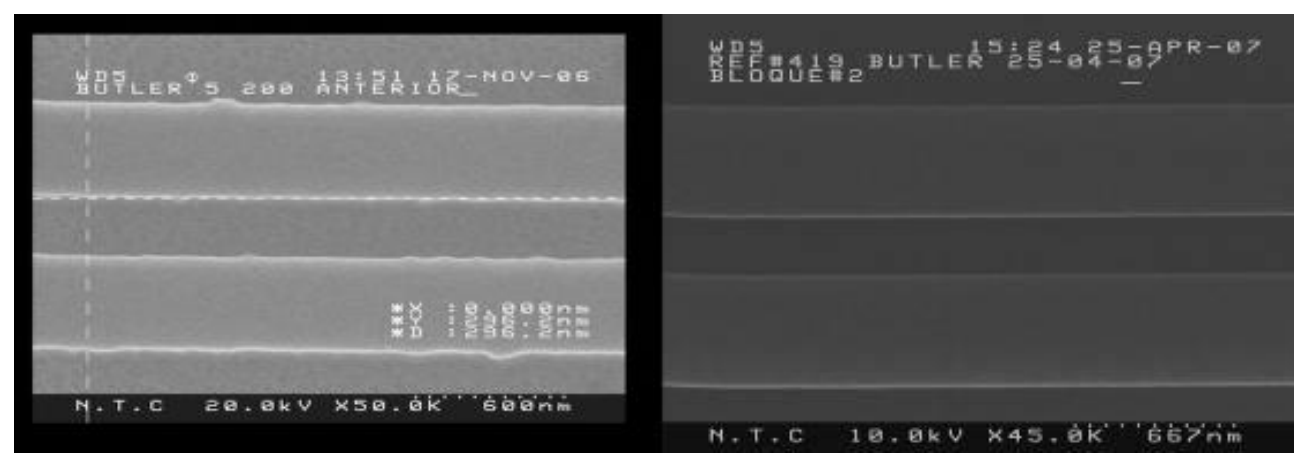

(a)

(b)

Figure B.9. Scanning electronic microscope (SEM) image of a coupler de fine d (a) with a standard process and (b) including proximity effect compensation. 


\section{Theore tical analysis of waveguide width variations on the phase shift error}

The influence of the waveguide width variations on the phase error has been thoroughly analyzed by means of simulations. The model shown in Figure B.10 has been used to characterize the phase error of one waveguide with respect to another after propagating a length of $L$. Ideally, the phases in the two waveguides are the same at each point. However, due to the variations of the waveguide width, there is a difference in the effective index and therefore a difference in the phases at each point of the two waveguides.

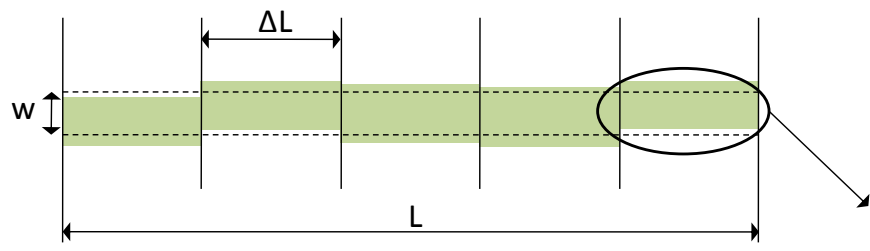

Case 1: $\Delta \mathrm{L}$ constant
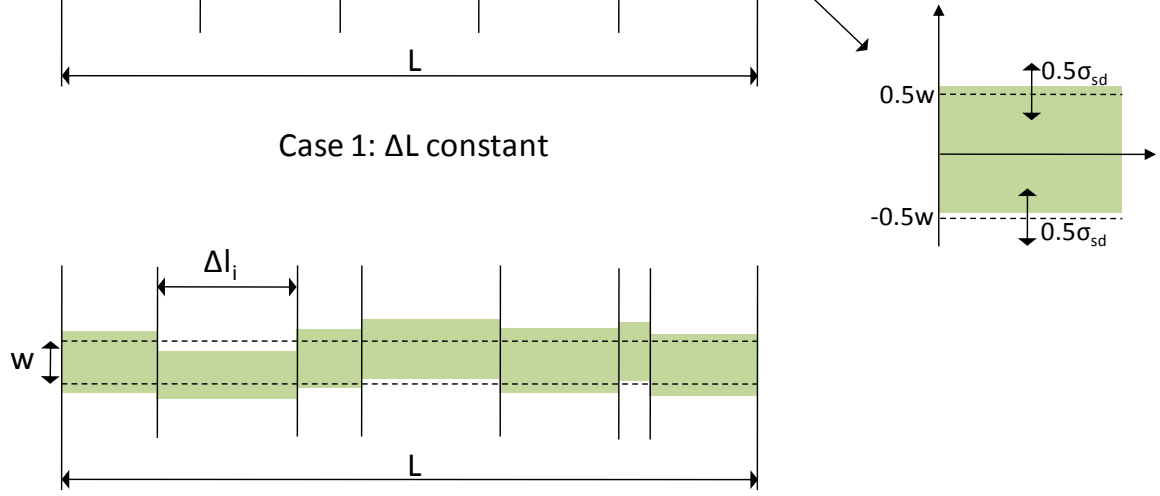

Case 2: $\Delta \mathrm{L}$ random $\left(\Delta \mathrm{I}_{\mathrm{i}} \leq \Delta \mathrm{I}_{\mathrm{MAX}}\right)$

\section{Figure B.10. Cases to analyze the influe nce of wave guide variations on the phase error.}

Two cases are considered: in the first case, the waveguides are formed by sections of constant length, $\Delta L$, while in the second case the waveguides are formed by random sections, so that the length of each section can uniformly vary from 0 to $\Delta L_{M A X}$. In each section, the variations of both sidewalls are uncorrelated, as it is depicted in Figure B.10, but they follow the same Gaussian distribution with $\sigma_{s d}$ standard deviation relative to the mean value $0.5 w=250 \mathrm{~nm}$. Thus, the variation of the waveguide width will also follow a Gaussian distribution with a standard deviation of $\sigma_{w g}=\sigma_{s d} / \sqrt{2}$. Waveguide width variations give rise to effective index variations so the phase error at a total length of $L$ will be: 


$$
\Delta \phi_{\text {error }}(L)=\Delta \phi_{i}(L)-\Delta \phi_{j}(L)=\frac{2 \pi}{\lambda}\left(\sum_{i} n_{e f f}^{i} \Delta L_{i}-\sum_{j} n_{e f f}^{j} \Delta L_{j}\right)
$$

where $\lambda=1550 \mathrm{~nm}$ is the operating wavelength. The variation of the effective index as a function of the waveguide width was calculated by a 3D mode solver based on the Beam Propagation Method (BPM). Phase error simulations were repeated a sufficient number of times in order to obtain the statistical parameters. The distribution of the phase error is also Gaussian with zero mean and a standard deviation that will depend on the length of the sections, $\Delta L$, the total length, $L$, and the standard deviation of the sidewall variations, $\sigma_{s d}$.

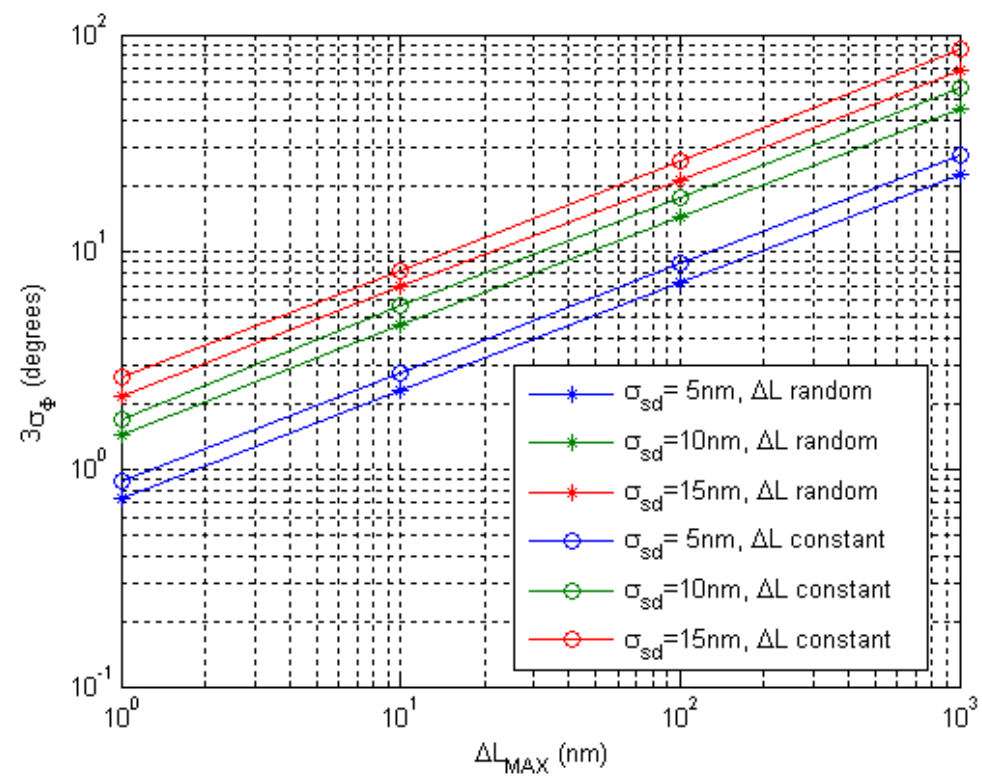

Figure B.11. Three times the standard deviation of the phase error as a function of the length of the sections, $\Delta L$, and the standard de viation of the side wall variations, $\sigma_{s d}$ for the two cases shown in Figure B.10. The total length, $L$, is $26 \mu \mathrm{m}$.

Figure B.11 shows the phase error when the total length is $L=26 \mu \mathrm{m}$, which is equal to the total length of the $8 \times 8$ Butler matrix without taking into account the directional couplers length. It has been considered three times the standard deviation to ensure that the phase error will be lower than this value with a probability of $99.72 \%$. First of all, it is interesting to see that the phase error for case 2 shown in Figure B.10 ( $\triangle L$ random) is lower than the phase error for case 1 ( $\triangle L$ constant). However, in both cases the phase error increases as the length of the sections, $\Delta L$, as 
well as the standard deviation of the sidewalls, $\sigma_{s d}$, increase. Furthermore, the variation of the phase error with the total length was also characterized and it was obtained that the phase error increased with length. Concretely, we obtained that the standard variation of the phase error has approximately the following relationship with the previous parameters:

$$
\sigma_{\phi} \alpha \frac{\sigma_{s d}}{\sqrt{2}} \sqrt{\Delta L} \sqrt{L} \equiv \sigma_{w g} \sqrt{\Delta L} \sqrt{L}
$$

which is in agreement with the theoretical model with constant $\Delta \mathrm{L}$ sections described in [DUM07]. Therefore, the total length of the Butler Matrix structure was minimized to reduce the phase error introduced by the variations of the waveguide width [SANO6].

In [SAN06] it was reported a phase error of 39 degrees for $\sigma_{\mathrm{wg}}=3.8 \mathrm{~nm}$. This phase error was calculated by using the following relation:

$$
\Delta \phi_{\text {error }}(L)=\frac{2 \pi}{\lambda} \Delta n_{\text {eff }} L=\frac{2 \pi}{\lambda}\left(n_{\text {eff }}(w, \lambda)-n_{\text {eff }}\left(w-\sigma_{\text {sd }}, \lambda\right)\right) L
$$

where $w$ is the target waveguide width. However, $\sigma_{w g}=3.8 \mathrm{~nm}$ corresponds to $\sigma_{s d}=5.37 \mathrm{~nm}$ so it was obtained that a maximum phase error of 39 degrees would only occur for $\Delta L$ values higher than $1.9 \mu \mathrm{m}$ for case 1 ( $\Delta L$ constant) and higher than $2.9 \mu \mathrm{m}$ for case $2(\Delta L$ random). In [DUM07], $\Delta L$ values in the range between $500 \mathrm{~nm}$ and $1 \mu \mathrm{m}$ were obtained for samples fabricated with deep-UV lithography.

The challenge with the proposed model is the calculation and/or measurement of the $\Delta L$ parameter. This was realized by two different methods. Firstly, by measuring different waveguide widths with an automatic measurement process of a SEM, and secondly also measuring the waveguide width by image post-processing of SEM images. $\Delta L$ was obtained by taking measurements of the waveguide width on a long distance (> $10 \mu \mathrm{m}$ with a step-size of $5 \mathrm{~nm}$ ) and then calculating the autocorrelation. The results for the $\Delta L$ approximations vary from 44 to $88 \mathrm{~nm}$ approximately depending on the measured waveguide. Figure B.12 shows the autocorrelation and the obtained values of $\Delta L$ for the two methods. Looking at Figure B.11, a phase shift standard deviation $\left(\sigma_{\varphi}\right)$ of around 5 degrees $(\Delta L$ random) and 6 degrees ( $\triangle L$ constant) will occur for $\Delta L=88.5 \mathrm{~nm}$ and for $\sigma_{s d}=10 \mathrm{~nm}$, which corresponds with the standard deviations of all the measured widths of all the $8 \times 8$ matrix samples fabricated. 

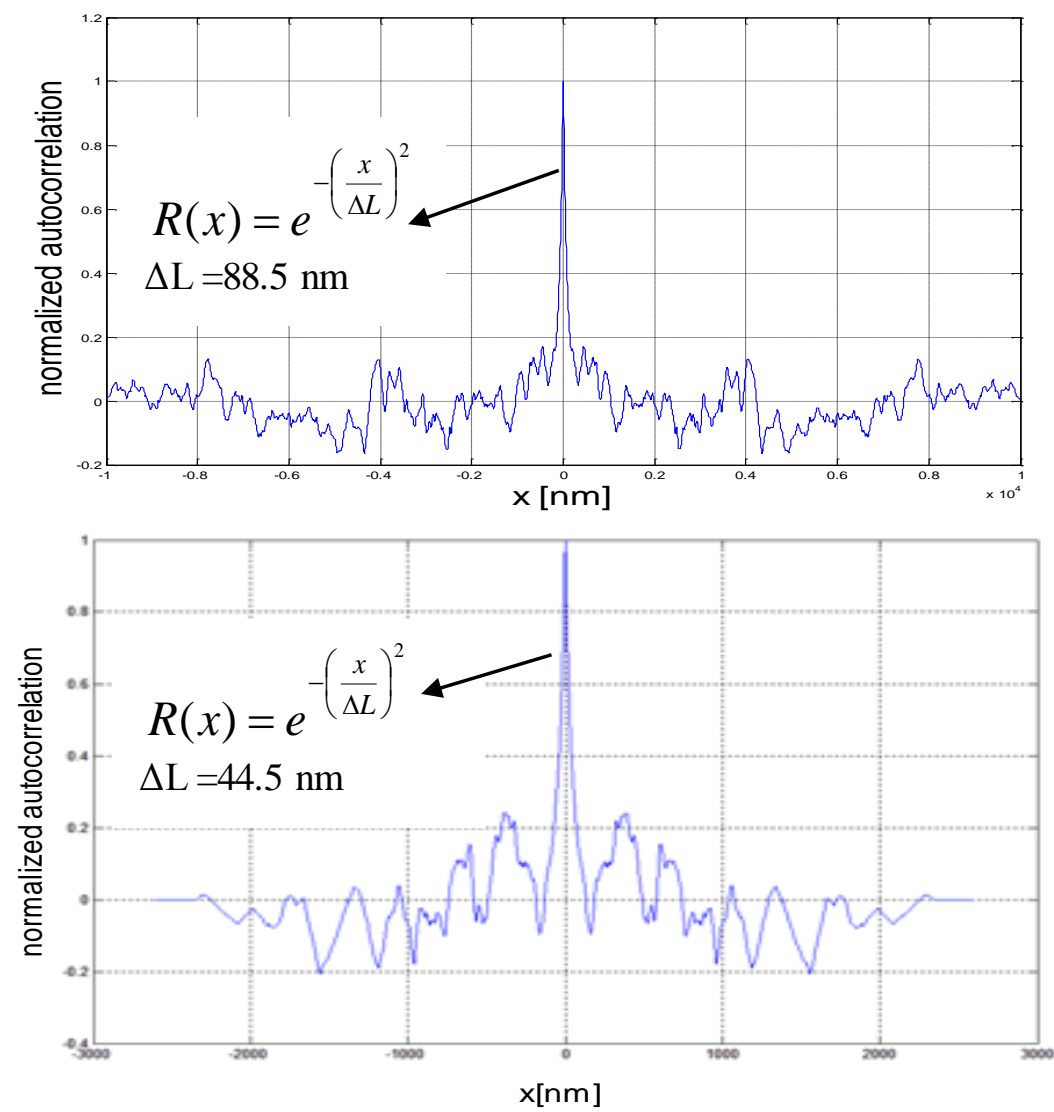

Figure B.12. Normalize d autocorre lation of the waveguide width variations measured with a an automatic measure ment process performed by a SEM (top) and with a image post-processing (bottom). 


\section{Annex C: Analytical model of the photonic vector modulator}

This annex describes the derivation of an analytical model for the photonic vector modulator architecture described in Chapter 8.

\section{C.1. Simplified PVM model}

Let it be the system depicted in Figure 98. Assuming no chirp, the optical field at the output of each laser is given by:

$$
\begin{aligned}
E_{\text {laser } 1} & =\sqrt{2\left(P_{1}+\eta_{1} x_{I}(t)\right)} e^{j\left(\Omega_{1} t+\varphi_{1}\right)} \\
E_{\text {laser } 2} & =\sqrt{2\left(P_{2}+\eta_{2} x_{Q}(t)\right)} e^{j\left(\Omega_{2} t+\varphi_{2}\right)}
\end{aligned}
$$

where $P_{i}$ is the output optical power at laser $i$ corresponding to the laser bias point $I_{\text {bias }}$ and $x_{I}(t)$ and $x_{Q}(t)$ are the baseband data signals ${ }^{18}$.

${ }^{18} \mathrm{It}$ is assumed that both data signals have zero mean value. Otherwise, these expressions should be considered: $x_{I}^{\prime}(t)=x_{I}(t)-\overline{x_{I}(t)} \quad x_{Q}{ }^{\prime}(t)=x_{Q}(t)-\overline{x_{Q}(t)}$

$$
P_{I}^{\prime}=P_{I}+\eta_{1} \overline{x_{I}(t)} \quad P_{Q}^{\prime}=P_{Q}+\eta_{2} \overline{x_{Q}(t)}
$$


At the MZM input both signals are combined:

$$
E_{\text {lasers }}=\sqrt{\frac{2\left(P_{1}+\eta_{1} x_{I}(t)\right)}{L_{1}}} e^{j\left(\Omega_{1} t+\varphi_{1}^{\prime}\right)}+\sqrt{\frac{2\left(P_{2}+\eta_{2} x_{Q}(t)\right)}{L_{2}}} e^{j\left(\Omega_{2} t+\varphi_{2}^{\prime}\right)}
$$

where $L_{1}$ and $L_{2}$ are the insertion losses between the lasers outputs and the MZM input. At the MZM output, assuming quadrature biasing $(\mathrm{QB})$, the field is given by expression (C.4) where $t_{f f}$ are the MZM insertion losses when the modulator is biased at maximum transmission biasing (MATB), ideally $t_{f f}=1$. The MZM behavior is assumed to be frequency independent with respect to $\Omega$.

$$
\begin{aligned}
& E_{m z m}(t)=\left[\sqrt{2 \frac{t_{f f}}{L_{1}}\left(P_{1}+\eta_{1} x_{I}(t-\tau)\right)} e^{j\left(\Omega_{1} t+\varphi_{1}^{\prime \prime}\right)}+\right. \\
& \left.\sqrt{2 \frac{t_{f f}}{L_{2}}\left(P_{2}+\eta_{2} x_{Q}(t-\tau)\right)} e^{j\left(\Omega_{2} t+\varphi_{2}^{\prime \prime}\right)}\right] \sum_{n=-\infty}^{\infty} J_{n}\left(m_{L O}\right) C_{D C}(n) e^{j\left(n \omega_{L O} t+n \varphi_{L O}\right)}
\end{aligned}
$$

where $C_{D C}(n)=\cos \left(\frac{\pi}{4} \pm n \frac{\pi}{2}\right)$ and $m_{L O}=\frac{\pi v_{L O}}{V_{\pi}}$.

If only the first terms are considered, the previous expression can be approximated by:

$$
\begin{aligned}
& E_{m z m}(t) \approx\left[J_{0}\left(m_{L O}\right) \pm 2 J_{1}\left(m_{L O}\right) \cos \left(\omega_{L O} t+\varphi_{L O}\right)\right] \\
& {\left[\sqrt{\frac{t_{f f}}{L_{1}}\left(P_{1}+\eta_{1} x_{I}(t-\tau)\right)} e^{j\left(\Omega_{1} t+\varphi_{1}^{*}\right)}+\sqrt{\frac{t_{f f}}{L_{2}}\left(P_{2}+\eta_{2} x_{Q}(t-\tau)\right)} e^{j\left(\Omega_{2} t+\varphi_{2}^{*}\right)}\right]}
\end{aligned}
$$

where the relation $J_{-I}\left(m_{L O}\right)=-J_{I}\left(m_{L O}\right)$ has been applied.

Assuming a simplified optical link which induces just a $\pi / 2$ radians phase $\operatorname{shift}^{19}$ at $f_{L O}$ between $\Omega_{1}$ and $\Omega_{2}$ with no further effect on $x_{I}(t), x_{Q}(t)$ or LO carrier; that is to say, $\Omega_{1}-\Omega_{2}$ is quite greater than the frequency contents of the baseband signals or the LO term.

${ }^{19}$ In order to obtain that phase shift by means of dispersion:

$$
L(\mathrm{~km})=\frac{250}{\Delta \lambda(\mathrm{nm}) \cdot D(\mathrm{ps} / \mathrm{nm} \cdot \mathrm{km}) f_{L O}(\mathrm{GHz})} \text { where } \Delta \lambda(\mathrm{nm})=\left|\frac{c}{\Omega_{1}}-\frac{c}{\Omega_{2}}\right|
$$




$$
\begin{aligned}
& E_{\text {link }}(t) \approx\left[\sqrt{\frac{t_{f f}}{L_{1} L_{f}}\left(P_{1}+\eta_{1} x_{I}\left(t-\tau^{\prime}\right)\right) e^{j\left(\Omega_{1} t+\varphi_{1}^{\prime}\right)}}\left[J_{0}\left(m_{L O}\right) \pm 2 J_{1}\left(m_{L O}\right) \cos \left(\omega_{L O} t+\varphi_{L O}^{l}\right)\right]+\right. \\
& \left.\sqrt{\frac{t_{f f}}{L_{2} L_{f}}\left(P_{2}+\eta_{2} x_{Q}\left(t-\tau^{\prime}-\frac{1}{4 f_{L O}}\right)\right)} e^{j\left(\Omega_{2} t+\varphi_{2}^{\prime}\right)}\left[J_{0}\left(m_{L O}\right) \pm 2 J_{1}\left(m_{L O}\right) \cos \left(\omega_{L O} t-\frac{\pi}{2}+\varphi_{L O}^{l}\right)\right]\right]
\end{aligned}
$$

Finally, the photocurrent can be calculated as:

$$
\begin{aligned}
& i_{p d}(t)=\frac{\mathfrak{R} E_{\text {link }} E_{\text {link }}^{*}}{2} \\
& \approx \frac{\mathfrak{R}}{2} \frac{t_{f f}}{L_{f}}\left[\left(\frac{P_{1}}{L_{1}}+\frac{\eta_{1}}{L_{1}} x_{I}\left(t-\tau^{\prime}\right)\right)\left[J_{0}\left(m_{L O}\right) \pm 2 J_{1}\left(m_{L O}\right) \cos \left(\omega_{L O} t+\varphi_{L O}^{l}\right)\right]^{2}\right. \\
& \left.+\left(\frac{P_{2}}{L_{2}}+\frac{\eta_{2}}{L_{2}} x_{Q}\left(t-\tau^{\prime}-\frac{1}{4 f_{L O}}\right)\right)\left[J_{0}\left(m_{L O}\right) \pm 2 J_{1}\left(m_{L O}\right) \cos \left(\omega_{L O} t-\frac{\pi}{2}+\varphi_{L O}^{l}\right)\right]^{2}\right]
\end{aligned}
$$

where the $\Omega_{1}-\Omega_{2}$ or $\Omega_{2} \Omega_{1}$ terms have been neglected.

The DC value of the detected current will be:

$$
i_{p d_{-} D C} \approx \frac{\Re}{2} \frac{t_{f f}}{L_{f}}\left(\frac{P_{1}}{L_{1}}+\frac{P_{2}}{L_{2}}\right)\left[J_{0}^{2}\left(m_{L O}\right)+2 J_{1}^{2}\left(m_{L O}\right)\right]
$$

or

$$
i_{p d_{-} D C} \approx \mathfrak{R} \frac{t_{f f} P}{L_{f} L}\left[J_{0}^{2}\left(m_{L O}\right)+2 J_{1}^{2}\left(m_{L O}\right)\right]
$$

if $P=P_{1}=P_{2}, L=L_{1}=L_{2}$.

Whereas, the detected signal around $f_{L O}$ will be:

$$
\begin{aligned}
i_{p d-L O}(t) \approx & \frac{\mathfrak{R} t_{f f}}{L_{f}} 2 J_{1}\left(m_{L O}\right) J_{0}\left(m_{L O}\right) \\
& {\left[\left(\frac{P_{1}}{L_{1}}+\frac{\eta_{1}}{L_{1}} x_{I}\left(t-\tau^{\prime}\right)\right) \cos \left(\omega_{L O} t+\varphi_{L O}^{l}\right)\right.} \\
& \left.+\left(\frac{P_{2}}{L_{2}}+\frac{\eta_{2}}{L_{2}} x_{Q}\left(t-\tau^{\prime}-\frac{1}{4 f_{L O}}\right)\right) \sin \left(\omega_{L O} t+\varphi_{L O}^{l}\right)\right]
\end{aligned}
$$

And the quadrature modulated RF signal is obtained. 
When $P=P_{1}=P_{2}, L=L_{1}=L_{2}, \eta=\eta_{1}=\eta_{2}$ and common delay/phase terms like $\tau^{\prime}$ or $\phi_{L O}{ }^{l}$ are neglected:

$$
\begin{aligned}
& i_{p d-L O}(t) \approx \frac{\mathfrak{R}}{L_{t o t}} 2 J_{1}\left(m_{L O}\right) J_{0}\left(m_{L O}\right) P \sqrt{2} \cos \left(\omega_{L O} t-\frac{\pi}{4}\right) \\
& +\frac{\Re}{L_{t o t}} 2 J_{1}\left(m_{L O}\right) J_{0}\left(m_{L O}\right) \eta\left[x_{I}(t) \cos \left(\omega_{L O} t\right)+x_{Q}\left(t-\frac{1}{4 f_{L O}}\right) \sin \left(\omega_{L O} t\right)\right]
\end{aligned}
$$

where $L_{t o t}$ are the optical losses from laser output to photodiode input when the MZM is biased at MATB (that is to say that the extra $3 \mathrm{~dB}$ due to QB are not considered in this value but in the rest of the equation) and where the MZM model only considers the two sidebands and no further harmonics of $f_{L O}$.

The detected LO carrier power (without data signals) assuming a load resistance equal to $R_{L}$ is given by:

$$
P_{p d-L O}=4 P^{2}\left[\frac{\mathfrak{R}}{L_{t o t}} J_{1}\left(m_{L O}\right) J_{0}\left(m_{L O}\right)\right]^{2} R_{L}
$$

On the other side, the detected signal power (if LO carrier were not present) assuming a load resistance equal to $R_{L}$ can be expressed as:

$$
P_{p d-\text { data }}=2 \eta^{2}\left(P_{I}+P_{Q}\right)\left[\frac{\Re}{L_{t o t}} J_{1}\left(m_{L O}\right) J_{0}\left(m_{L O}\right)\right]^{2} R_{L}
$$

where $P_{I}=\left\langle x_{I}^{2}(t)\right\rangle$ and $P_{Q}=\left\langle x_{Q}^{2}(t)\right\rangle$.

Thus, the relation between signal power and carrier power is:

$$
\frac{P_{p d-L O}}{P_{p d-\text { data }}}=\frac{2 P^{2}}{\eta^{2}\left(P_{I}+P_{Q}\right)}
$$

If both $\mathrm{LO}$ carrier and data are present the total power around $f_{L O}$ is:

$$
P_{p d-L O \_d a t a}=P_{p d-L O}+P_{p d-d a t a}+2 P \eta\left[\frac{\Re}{L_{t o t}} J_{1}\left(m_{L O}\right) J_{0}\left(m_{L O}\right)\right]^{2}\left[\left(\overline{x_{I}(t)}+\overline{x_{Q}(t)}\right)\right] R_{L}
$$

Some limitations may be identified:

- There is a small delay in $x_{Q}$ with respect to $x_{I}$ which can be neglected as long as $f_{L O}$ is quite greater than the symbol rate. When the I-branch wavelength is 
delayed by $\left(1 / 4 f_{L O}\right)$ with respect to the Q-branch wavelength, the corresponding baseband signals are also delayed. If $f_{L O}=20 \mathrm{GHz}$ and baseband data rate is $1 \mathrm{~Gb} / \mathrm{s}$ per branch ( $2 \mathrm{~Gb} / \mathrm{s}$ totally), the delay is $12.5 \mathrm{ps}$ while the symbol rate is 1,000 ps so a $1.25 \%$ differential time shifting is induced between I and Q demodulated branches. In any case, this time shift could be corrected by inserting a differential delay in the optical path between both lasers and the combiner or by inserting a differential electrical delay between the digital streams before driving the lasers.

- The bias level of the laser diodes $\left(P_{1}\right.$ or $\left.P_{2}\right)$ is translated to a constant amount of RF carrier which implies a waste or RF power. If the digital baseband signals are just ideal NRZ signals corresponding to two optical power levels at the laser output $\left(P_{O N}\right.$ and $\left.P_{O F F}\right)$ the ratio between the LO power and the data power at the photodetector output is ${ }^{20}$ :

$$
\frac{P_{p d-L O}}{P_{p d-\text { data }}}=\frac{2 P^{2}}{\eta^{2}\left(P_{I}+P_{Q}\right)}=\left(\frac{P_{O N}+P_{O F F}}{P_{O N}-P_{O F F}}\right)^{2}
$$

Which is graphically plotted in Figure C.1.

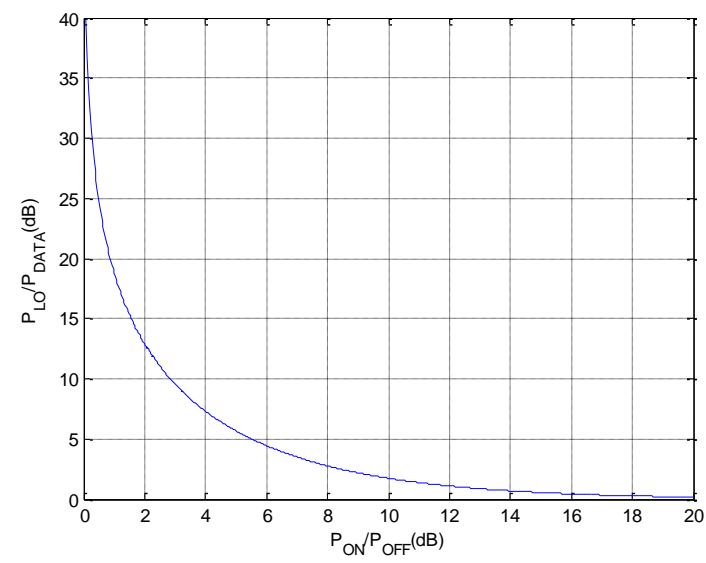

Figure C.1. Detected LO/Data power ratio (dB) as a function of las er $\mathrm{P}_{\mathrm{ON}} / \mathrm{P}_{\text {OFF }}$ ratio.

${ }^{20}$ If $x_{I}(t)$ and $x_{Q}(t)$ were two digital bipolar signals $\left(I_{\text {peak }},-I_{\text {peak }} \ldots\right.$ randomly) with equal probability for both levels, then the mean optical power at each laser, $P$ :

$$
P_{I}=P_{Q}=I_{\text {peak }}^{2} \quad P=\eta I_{\text {bias }} \quad P_{O N}=\eta\left(I_{\text {bias }}+I_{\text {peak }}\right) \quad P_{\text {OFF }}=\eta\left(I_{\text {bias }}-I_{\text {peak }}\right)
$$

Thus,

$$
P=\frac{P_{O N}+P_{O F F}}{2} \quad P_{O N}-P_{O F F}=2 \eta I_{\text {peak }}
$$


Thus, in the best case (i.e. when $P_{O N}$ is quite larger than $P_{O F F}$ ), the RF power wasted at $\mathrm{LO}$ carrier is equal to the data power, that is to say, $3 \mathrm{~dB}$ of dynamic range is wasted to accommodate the carrier power. In a more realistic scenario, if $P_{O N}$ is $8 \mathrm{~dB}$ greater than the $P_{\text {OFF }}$ optical level then the RF power at the LO carrier is $3 \mathrm{~dB}$ greater than the data power at the photodetector output.

\section{C.2. Noise analysis}

In this section, the different noise sources in the PVM system described in Chapter 8 are revised. The main noise sources were described in Section 1.4.

○ Thermal (See section 1.4.1.1)

$$
\sigma_{\text {thermal }}^{2}=\frac{4 k T \Delta f}{R_{L}}
$$

(or $\sigma_{\text {thermal }}^{2}=\frac{4 k T \Delta f}{R_{L}} F$ if the noise factor of the photoreceiver preamplifier is included).

- Shot noise (see section 1.4.1.2)

$$
\sigma_{\text {shot }}^{2}=2 q\left(\frac{\mathfrak{R P}}{L_{\text {tot }}}\left[J_{0}^{2}\left(m_{L O}\right)+2 J_{1}^{2}\left(m_{L O}\right)\right]+i_{\text {dark }}\right) \Delta f
$$

○ RIN (See section 1.4.1.3)

$$
\sigma_{R I N}^{2}=R I N\left(\frac{\Re P}{L_{t o t}}\left[J_{0}^{2}\left(m_{L O}\right)+2 J_{1}^{2}\left(m_{L O}\right)\right]\right)^{2} \Delta f
$$

The total noise is given by:

$$
\begin{aligned}
& \frac{S}{N}=\frac{P_{p d-\text { data }}}{\sigma_{R I N}^{2} R_{L}+\sigma_{\text {shot }}^{2} R_{L}+\sigma_{\text {thermal }}^{2} R_{L}}= \\
& \frac{2 \eta^{2}\left(P_{I}+P_{Q}\right)\left[\frac{\Re}{L_{\text {tot }}} J_{1}\left(m_{L O}\right) J_{0}\left(m_{L O}\right)\right]^{2}}{\left[R I N\left(\Re P \frac{J_{0}^{2}\left(m_{L O}\right)+2 J_{1}^{2}\left(m_{L O}\right)}{L_{\text {tot }}}\right)^{2}+2 q\left(\Re P \frac{J_{0}^{2}\left(m_{L O}\right)+2 J_{1}^{2}\left(m_{L O}\right)}{L_{\text {tot }}}+i_{\text {dark }}\right)+\frac{4 k T}{R_{L}}\right] \Delta f}
\end{aligned}
$$

The relative contribution of each type of noise is represented in the Figure C.2. 

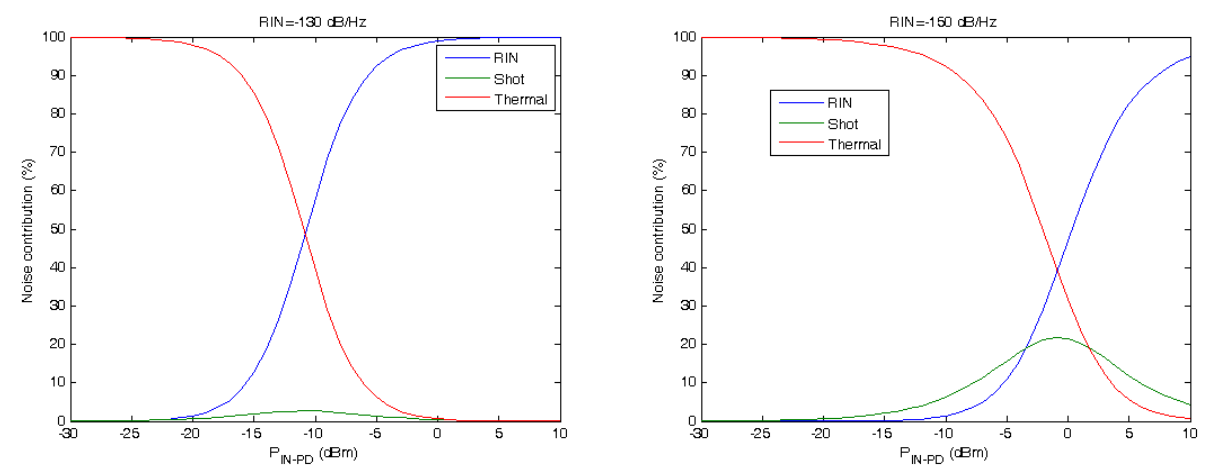

Figure C.2. PVM noise contributions as a function of received optical power.

In a RIN-dominated scenario, it is possible to calculate the signal to noise ratio as:

$$
\begin{aligned}
\frac{S}{N}= & \frac{P_{p d-\text { data }}}{\sigma_{R I N}^{2} R_{L}}=\frac{2 \eta^{2}\left(P_{I}+P_{Q}\right)}{P^{2}} \frac{1}{R I N \Delta f} \frac{\left[J_{1}\left(m_{L O}\right) J_{0}\left(m_{L O}\right)\right]^{2}}{\left[J_{0}^{2}\left(m_{L O}\right)+2 J_{1}^{2}\left(m_{L O}\right)\right]^{2}} \\
= & 4 \frac{P_{p d-\text { data }}}{P_{p d-L O}} \frac{1}{R I N \Delta f} \frac{\left[J_{1}\left(m_{L O}\right) J_{0}\left(m_{L O}\right)\right]^{2}}{\left[J_{0}^{2}\left(m_{L O}\right)+2 J_{1}^{2}\left(m_{L O}\right)\right]^{2}} \\
\frac{S}{N}(d B)= & 6-\frac{P_{p d-L O}}{P_{p d-\text { data }}}(d B)-R I N(d B / H z) \\
& -10 \log _{10}[\Delta f(H z)]+\text { Factor }_{L O}(d B)
\end{aligned}
$$

where

$$
\text { Factor }_{L O}(d B)=20 \log _{10}\left(\frac{J_{1}\left(m_{L O}\right) J_{0}\left(m_{L O}\right)}{J_{0}^{2}\left(m_{L O}\right)+2 J_{1}^{2}\left(m_{L O}\right)}\right)
$$

expression that is plotted in Figure C.3. 


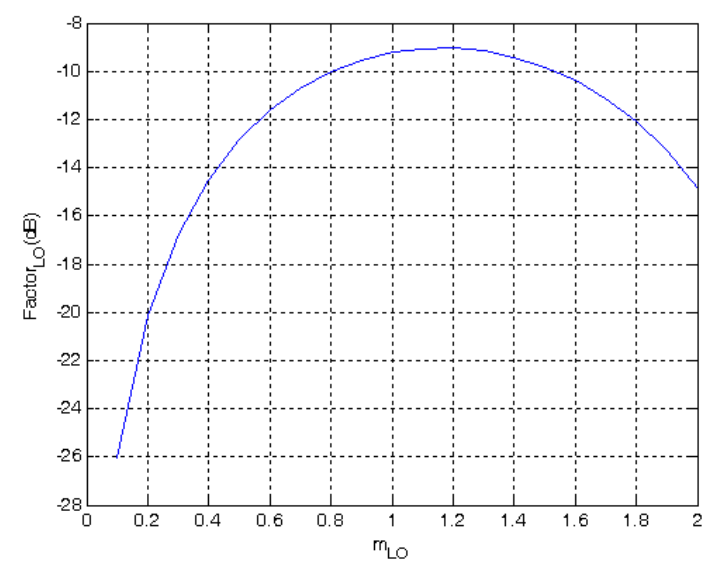

Figure C.3. Dependence of the Factor LO $_{\text {on }}$ the modulation index.

For instance, RIN=-130 dB/Hz, $\Delta f=1 \mathrm{e} 9 \mathrm{~Hz}$ (corresponds to the bandwidth needed for a $2 \mathrm{~Gb} / \mathrm{s}$ QPSK with root raised cosine $\alpha=0$ ), best Factor $_{\mathrm{LO}}=-9 \mathrm{~dB}$ and a ratio $\mathrm{P}_{\mathrm{LO}} / \mathrm{P}_{\mathrm{data}}=3 \mathrm{~dB}$ then,

$$
\frac{S}{N}=6-3+130-90-9=32 d B
$$

\section{C.3. Complete PVM model with a second-order dispersive link (QB biasing)}

In this section, the previous assumption of a simplified link inducing a $\pi / 2$ phase shift between both optical carriers is proved by studying the chromatic dispersion of the optical link up to its second order.

Let it be a dispersive link with the spectral response given by:

$$
H(\Omega)=\frac{1}{\sqrt{L_{f}}} e^{j \beta(\Omega) L}
$$

Chromatic dispersion can be approximated by a Taylor expansion that, in a first approach, can be trunked in the $\beta_{2}$ term.

$$
\begin{gathered}
\beta(\Omega)=\beta_{0}+\beta_{1}\left(\Omega-\Omega_{0}\right)+\frac{\beta_{2}}{2}\left(\Omega-\Omega_{0}\right)^{2} \\
\frac{d \beta}{d \Omega}=\beta_{1}+\beta_{2}\left(\Omega-\Omega_{0}\right)
\end{gathered}
$$


where $\beta_{1}$ is the group delay at $\Omega_{0}$, and $\beta_{2}$ is related to the dispersion parameter of the fibre as

$$
\begin{gathered}
\beta_{2}=G V D=\frac{d^{2} \beta}{d \Omega^{2}} \\
\beta_{2}=-\frac{D \lambda_{0}^{2}}{2 \pi c}
\end{gathered}
$$

As $D$ is positive in standard single-mode fibre (SSMF) for wavelengths in the anomalous dispersion region above $1276 \mathrm{~nm}$, then $\beta_{2}$ is negative in that region. Then, when comparing two optical carriers, $\Omega_{1} / \Omega_{2}$, above $1276 \mathrm{~nm}$, the higher $\Omega$ (in THz), (that is to say, the lower in $\mathrm{nm}$ ) will have lower group delay,

$$
\Delta \Omega=-\frac{2 \pi c}{\lambda^{2}} \Delta \lambda
$$

In the quadrature condition,

$$
L \beta_{2} \Delta \Omega=\frac{1}{4 f_{L O}}
$$

Then $-L \beta_{2} \frac{2 \pi c}{\lambda^{2}} \Delta \lambda=\frac{1}{4 f_{L O}}$ and finally:

$$
D L \Delta \lambda=\frac{1}{4 f_{L O}}
$$

The combination of the previous results, i.e. the MZM model with just carrier and sidebands, QB biasing and $L_{1}=L_{2}=L$ with dispersive link up to $\beta_{2}$ provides:

$$
E_{m z m}(t) \approx A\left[B \pm C \cos \left(\omega_{L O} t+\varphi_{L O}\right)\right]\left[I(t) e^{j\left(\Omega_{1} t+\varphi_{1}^{\prime \prime}\right)}+Q(t) e^{j\left(\Omega_{2} t+\varphi_{2}^{\prime \prime}\right)}\right]
$$

where

$$
\begin{array}{llr}
A=\sqrt{\frac{t_{f f}}{L}} \quad B=J_{0}\left(m_{L O}\right) & C=2 J_{1}\left(m_{L O}\right) \\
I(t)=\sqrt{P+\eta x_{I}(t-\tau)} & Q(t)=\sqrt{P+\eta x_{Q}(t-\tau)}
\end{array}
$$

Equation (C.33) can be expressed as: 


$$
\begin{gathered}
E_{m z m}(t) \approx A\left[B \pm \frac{C}{2} e^{j\left(\omega_{L O} t+\varphi_{L O}\right)} \pm \frac{C}{2} e^{-j\left(\omega_{L D} t+\varphi_{L O}\right)}\right] \\
{\left[I(t) e^{j\left(\Omega_{1} t+\varphi_{1}^{\prime \prime}\right)}+Q(t) e^{j\left(\Omega_{2} t+\varphi_{2}^{\prime \prime}\right)}\right]}
\end{gathered}
$$

assuming that the electrical and optical phase play no role, neither the sign ambiguity,

$$
\begin{aligned}
E_{m z m}(t) & \approx A I(t)\left[B e^{j \Omega_{1} t}+\frac{C}{2} e^{j\left(\Omega_{1}+\omega_{L O}\right) t}+\frac{C}{2} e^{j\left(\Omega_{1}-\omega_{L O}\right) t}\right] \\
& +A Q(t)\left[B e^{j \Omega_{2} t}+\frac{C}{2} e^{j\left(\Omega_{2}+\omega_{L O}\right) t}+\frac{C}{2} e^{j\left(\Omega_{2}-\omega_{L O}\right) t}\right]
\end{aligned}
$$

After the dispersive link of length $L$ and neglecting the effect of dispersion on $I(t)$ or $Q(t)$, that is to say assuming their frequency content is small compared to the dispersion of the link, the expression (C.38) can be obtained. However, the dispersion of the link is delaying $Q(t)$ respect to $I(t)$ in the same way as the corresponding LO terms, the only way to model this is assuming that $I(t)$ and $Q(t)$ are delayed according to the group delays at $\Omega_{1}$ and $\Omega_{2}$ :

$$
\begin{gathered}
I^{\prime}(t)=I\left(t+\beta_{1} L+\beta_{2} L\left(\Omega_{1}-\Omega_{0}\right)\right) \\
Q^{\prime}(t)=Q\left(t+\beta_{1} L+\beta_{2} L\left(\Omega_{2}-\Omega_{0}\right)\right) \\
E_{\text {link }}(t) \approx E_{m z m}(t) e^{j \beta(\Omega) L} \\
=A^{\prime} I^{\prime}(t) e^{j \beta_{0} L}\left[\begin{array}{l}
B e^{j \Omega_{1} t} e^{j \beta_{1} L\left(\Omega_{1}-\Omega_{0}\right)} e^{j \frac{\beta_{2} L}{2}\left(\Omega_{1}-\Omega_{0}\right)^{2}} \\
+\frac{C}{2} e^{j\left(\Omega_{1}+\omega_{L O}\right) t} e^{j \beta_{1} L\left(\Omega_{1}+\omega_{L O}-\Omega_{0}\right)} e^{j \frac{\beta_{2} L}{2}\left(\Omega_{1}+\omega_{L O}-\Omega_{0}\right)^{2}} \\
+\frac{C}{2} e^{j\left(\Omega_{1}-\omega_{L O}\right) t} e^{j \beta_{1} L\left(\Omega_{1}-\omega_{L O}-\Omega_{0}\right)} e^{j \frac{\beta_{2} L}{2}\left(\Omega_{1}-\omega_{L O}-\Omega_{0}\right)^{2}}
\end{array}\right] \\
+A^{\prime} Q^{\prime}(t) e^{j \beta_{0} L}\left[\begin{array}{l}
B e^{j \Omega_{2} t} e^{j \beta_{1} L\left(\Omega_{2}-\Omega_{0}\right)} e^{j \frac{\beta_{2} L}{2}\left(\Omega_{2}-\Omega_{0}\right)^{2}} \\
+\frac{C}{2} e^{j\left(\Omega_{2}+\omega_{L O}\right) t} e^{j \beta_{1} L\left(\Omega_{2}+\omega_{L O}-\Omega_{0}\right)} e^{j \frac{\beta_{2} L}{2}\left(\Omega_{2}+\omega_{L O}-\Omega_{0}\right)^{2}} \\
+\frac{C}{2} e^{j\left(\Omega_{2}-\omega_{L O}\right) t} e^{j \beta_{1} L\left(\Omega_{2}-\omega_{L O}-\Omega_{0}\right)} e^{j \frac{\beta_{2} L}{2}\left(\Omega_{2}-\omega_{L O}-\Omega_{0}\right)^{2}}
\end{array}\right]
\end{gathered}
$$


where $A^{\prime}=\sqrt{t_{f f} /\left(L_{f} L\right)}$.

After some algebra, following expression can be obtained for the optical field impinging the photoreceiver:

$$
\begin{aligned}
E_{\text {link }}(t) \approx & A^{\prime} I^{\prime}(t) e^{j \beta_{0} L} e^{j \Omega_{1} t} e^{j \beta_{1} L\left(\Omega_{1}-\Omega_{0}\right)} e^{j \frac{\beta_{2} L}{2}\left(\Omega_{1}-\Omega_{0}\right)^{2}} \\
& {\left[B+e^{j \frac{\beta_{2} L}{2} \omega_{L O}^{2}} C \cos \left(\omega_{L O}\left(t+\beta_{1} L\right)+\beta_{2} L\left(\Omega_{1}-\Omega_{0}\right) \omega_{L O}\right)\right] } \\
& +A^{\prime} Q^{\prime}(t) e^{j \beta_{0} L} e^{j \Omega_{2} t} e^{j \beta_{1} L\left(\Omega_{2}-\Omega_{0}\right)} e^{j \frac{\beta_{2} L}{2}\left(\Omega_{2}-\Omega_{0}\right)^{2}} \\
& {\left[B+e^{j \frac{\beta_{2} L}{2} \omega_{L O}^{2}} C \cos \left(\omega_{L O}\left(t+\beta_{1} L\right)+\beta_{2} L\left(\Omega_{2}-\Omega_{0}\right) \omega_{L O}\right)\right] }
\end{aligned}
$$

And the photocurrent can be calculated as:

$$
\begin{aligned}
& i_{\text {pd }}(t)=\frac{\mathfrak{R} E_{\text {link }} E_{\text {link }}^{*}}{2}=\frac{\mathfrak{R} A^{\prime 2}}{2} \\
& {\left[I^{\prime 2}(t)\left[\begin{array}{l}
2 B C \cos \left(\frac{\beta_{2} L}{2} \omega_{L O}^{2}\right) \cos \left(\omega_{L O}\left(t+\beta_{1} L\right)+\beta_{2} L\left(\Omega_{1}-\Omega_{0}\right) \omega_{L O}\right) \\
B^{2}++C^{2} \cos ^{2}\left(\omega_{L O}\left(t+\beta_{1} L\right)+\beta_{2} L\left(\Omega_{1}-\Omega_{0}\right) \omega_{L O}\right)
\end{array}\right]\right.} \\
& \left.+Q^{\prime 2}(t)\left[\begin{array}{l}
2 B C \cos \left(\frac{\beta_{2} L}{2} \omega_{L O}^{2}\right) \cos \left(\omega_{L O}\left(t+\beta_{1} L\right)+\beta_{2} L\left(\Omega_{2}-\Omega_{0}\right) \omega_{L O}\right) \\
B^{2}++C^{2} \cos ^{2}\left(\omega_{L O}\left(t+\beta_{1} L\right)+\beta_{2} L\left(\Omega_{2}-\Omega_{0}\right) \omega_{L O}\right)
\end{array}\right]\right]
\end{aligned}
$$

where the $+/-\Omega_{1}+/-\Omega_{2}$ terms have been neglected. Finally, the detected current around $f_{L O}$ is:

$$
\begin{aligned}
& i_{p d_{-} L O}(t)=\mathfrak{R} A^{\prime 2} B C \cos \left(\frac{\beta_{2} L}{2} \omega_{L O}^{2}\right) \\
& {\left[I^{2}\left(t+\beta_{1} L+\beta_{2} L\left(\Omega_{1}-\Omega_{0}\right)\right) \cos \left(\omega_{L O}\left(t+\beta_{1} L+\beta_{2} L\left(\Omega_{1}-\Omega_{0}\right)\right)\right)\right.} \\
& \left.+Q^{2}\left(t+\beta_{1} L+\beta_{2} L\left(\Omega_{2}-\Omega_{0}\right)\right) \cos \left(\omega_{L O}\left(t+\beta_{1} L+\beta_{2} L\left(\Omega_{2}-\Omega_{0}\right)\right)\right)\right]
\end{aligned}
$$

According to this result, $I(t)$ and its LO carrier are delayed according to $\tau_{1}=-\beta_{1} L-\beta_{2} L\left(\Omega_{1}-\Omega_{0}\right)$ whereas $Q(t)$ and its $L O$ carrier are delayed according to 
$\tau_{2}=-\beta_{1} L-\beta_{2} L\left(\Omega_{2}-\Omega_{0}\right)$, that is to say, the $\mathrm{Q}$ component is relatively delayed with respect to the I carrier by,

$$
\tau_{21}=\tau_{2}-\tau_{1}=-\beta_{2} L\left(\Omega_{2}-\Omega_{1}\right)
$$

which is a positive value for wavelength above $1276 \mathrm{~nm}$ and when $\Omega_{2}>\Omega_{1}$ and substituting the values,

$$
\begin{aligned}
& i_{p d_{-} L O}(t)=\Re \frac{1}{L_{t o t}} J_{0}\left(m_{L O}\right) 2 J_{1}\left(m_{L O}\right) \cos \left(\frac{\beta_{2} L}{2} \omega_{L O}^{2}\right) \\
& {\left[\left[P+\eta x_{I}(t)\right] \cos \left(\omega_{L O} t\right)+\left[P+\eta x_{Q}\left(t-\tau_{21}\right)\right] \cos \left(\omega_{L O}\left(t-\tau_{21}\right)\right)\right]}
\end{aligned}
$$

where $L_{t o t}$ are the optical losses from laser output to photodiode input when the MZM is biased as its maximum transmission point (that is to say that the $3 \mathrm{~dB}$ extra due to QB are not considered in this value but in the rest of the equation) and where the MZM model has only considered the two sidebands and no further harmonics of $f_{L O}$.

The term $\tau_{21}=-\beta_{2} L\left(\Omega_{2}-\Omega_{1}\right)$ is the differential delay induced in the carriers due to fibre dispersion. When the length of the fibre and the wavelengths spacing are choose to fulfill the following equation (quadrature condition):

then

$$
\tau_{21}=-\beta_{2} L\left(\Omega_{2}-\Omega_{1}\right)=\frac{\tau_{L O}}{4}=\frac{1}{4 f_{L O}}
$$

$$
\begin{aligned}
& i_{p d_{-} L O}(t)=\mathfrak{R} \frac{1}{L_{t o t}} J_{0}\left(m_{L O}\right) 2 J_{1}\left(m_{L O}\right) \cos \left(\frac{\beta_{2} L}{2} \omega_{L O}^{2}\right) \\
& {\left[\left[P+\eta x_{I}(t)\right] \cos \left(\omega_{L O} t\right)+\left[P+\eta x_{Q}\left(t-\frac{1}{4 f_{L O}}\right)\right] \cos \left(\omega_{L O} t-\frac{\pi}{2}\right)\right]^{(C .}}
\end{aligned}
$$

Finally, the LO component is made of a fixed LO carrier and an ideal QAM signal,

$$
\begin{aligned}
& i_{p d_{-} L O}(t) \approx \frac{2 \Re J_{0}\left(m_{L O}\right) J_{1}\left(m_{L O}\right)}{L_{t o t}} \\
& {\left[P \sqrt{2} \cos \left(\omega_{L O} t-\frac{\pi}{4}\right)+\eta x_{I}(t) \cos \left(\omega_{L O} t\right)+\eta x_{Q}(t) \sin \left(\omega_{L O} t\right)\right]}
\end{aligned}
$$




\section{C.4. Complete model with a second-order dispersive link (MATB biasing)}

When the MZM is biased at its maximum transmission biasing (MATB) equations (C.34) and (C.35) change to:

$$
E_{m z m}(t) \approx A\left[B \pm C \cos \left(\omega_{L O} t+\phi_{L O}\right)\right]\left[I(t) e^{j\left(\Omega_{1} t+\phi_{1}^{\prime \prime}\right)}+Q(t) e^{j\left(\Omega_{2} t+\phi_{2}^{\prime \prime}\right)}\right]
$$

where

$$
\begin{array}{ccc}
A=\sqrt{2 \frac{t_{f f}}{L}} \quad B=0 & C=2 J_{1}\left(m_{L O}\right) \\
I(t)=\sqrt{P+\eta x_{I}(t-\tau)} & Q(t)=\sqrt{P+\eta x_{Q}(t-\tau)}
\end{array}
$$

And the optical field at the input of the photodiode can be expressed as:

$$
\begin{aligned}
& E_{\text {link }}(t) \approx E_{m z m}(t) e^{j \beta(\Omega) L} \\
& =A^{*} I^{\prime}(t) e^{j \beta_{0} L}\left[\begin{array}{l}
B e^{j \Omega_{1} t} e^{j \beta_{1} L\left(\Omega_{1}-\Omega_{0}\right)} e^{j \frac{\beta_{2} L}{2}\left(\Omega_{1}-\Omega_{0}\right)^{2}} \\
+\frac{C}{2} e^{j\left(\Omega_{1}+\omega_{L O}\right) t} e^{j \beta_{1} L\left(\Omega_{1}+\omega_{L O}-\Omega_{0}\right)} e^{j \frac{\beta_{2} L}{2}\left(\Omega_{1}+\omega_{L O}-\Omega_{0}\right)^{2}} \\
+\frac{C}{2} e^{j\left(\Omega_{1}-\omega_{L O}\right) t} e^{j \beta_{1} L\left(\Omega_{1}-\omega_{L O}-\Omega_{0}\right)} e^{j \frac{\beta_{2} L}{2}\left(\Omega_{1}-\omega_{L O}-\Omega_{0}\right)^{2}}
\end{array}\right] \\
& +A^{*} Q^{\prime}(t) e^{j \beta_{0} L}\left[\begin{array}{l}
B e^{j \Omega_{2} t} e^{j \beta_{1} L\left(\Omega_{2}-\Omega_{0}\right)} e^{j \frac{\beta_{2} L}{2}\left(\Omega_{2}-\Omega_{0}\right)^{2}} \\
+\frac{C}{2} e^{j\left(\Omega_{2}+\omega_{L O}\right) t} e^{j \beta_{1} L\left(\Omega_{2}+\omega_{L O}-\Omega_{0}\right)} e^{j \frac{\beta_{2} L}{2}\left(\Omega_{2}+\omega_{L O}-\Omega_{0}\right)^{2}} \\
+\frac{C}{2} e^{j\left(\Omega_{2}-\omega_{L O}\right) t} e^{j \beta_{1} L\left(\Omega_{2}-\omega_{L O}-\Omega_{0}\right)} e^{j \frac{\beta_{2} L}{2}\left(\Omega_{2}-\omega_{L O}-\Omega_{0}\right)^{2}}
\end{array}\right]
\end{aligned}
$$

where $A^{*}=\sqrt{2 t_{f f} /\left(L_{f} L\right)}$.

After some algebra, following expression can be obtained for the optical field impinging the photoreceiver: 


$$
\begin{aligned}
E_{\text {link }}(t) \approx & A^{\prime} I^{\prime}(t) e^{j \beta_{0} L} e^{j \Omega_{1} t} e^{j \beta_{1} L\left(\Omega_{1}-\Omega_{0}\right)} e^{j \frac{\beta_{2} L}{2}\left(\Omega_{1}-\Omega_{0}\right)^{2}} \\
& {\left[B+e^{j \frac{\beta_{2} L}{2} \omega_{L O}^{2}} C \cos \left(\omega_{L O}\left(t+\beta_{1} L\right)+\beta_{2} L\left(\Omega_{1}-\Omega_{0}\right) \omega_{L O}\right)\right] } \\
& +A^{\prime} Q^{\prime}(t) e^{j \beta_{0} L} e^{j \Omega_{2} t} e^{j \beta_{1} L\left(\Omega_{2}-\Omega_{0}\right)} e^{j \frac{\beta_{2} L}{2}\left(\Omega_{2}-\Omega_{0}\right)^{2}} \\
& {\left[B+e^{j \frac{\beta_{2} L}{2} \omega_{L O}^{2}} C \cos \left(\omega_{L O}\left(t+\beta_{1} L\right)+\beta_{2} L\left(\Omega_{2}-\Omega_{0}\right) \omega_{L O}\right)\right] }
\end{aligned}
$$

And the photocurrent can be expressed as:

$$
\begin{aligned}
& i_{p d}(t)=\frac{\Re E_{\text {link }} E_{\text {link }}^{*}}{2}=\frac{\Re A^{* 2}}{2} \\
& {\left[\begin{array}{l}
I^{\prime 2}(t)\left[\begin{array}{l}
B^{2}+2 B C \cos \left(\frac{\beta_{2} L}{2} \omega_{L O}^{2}\right) \cos \left(\omega_{L O}\left(t+\beta_{1} L\right)+\beta_{2} L\left(\Omega_{1}-\Omega_{0}\right) \omega_{L O}\right) \\
+C^{2} \cos ^{2}\left(\omega_{L O}\left(t+\beta_{1} L\right)+\beta_{2} L\left(\Omega_{1}-\Omega_{0}\right) \omega_{L O}\right)
\end{array}\right] \\
+Q^{\prime 2}(t)\left[\begin{array}{l}
B^{2}+2 B C \cos \left(\frac{\beta_{2} L}{2} \omega_{L O}^{2}\right) \cos \left(\omega_{L O}\left(t+\beta_{1} L\right)+\beta_{2} L\left(\Omega_{2}-\Omega_{0}\right) \omega_{L O}\right) \\
+C^{2} \cos ^{2}\left(\omega_{L O}\left(t+\beta_{1} L\right)+\beta_{2} L\left(\Omega_{2}-\Omega_{0}\right) \omega_{L O}\right)
\end{array}\right]
\end{array}\right]}
\end{aligned}
$$

where the $\pm\left(\Omega_{1} \pm \Omega_{2}\right)$ terms have been neglected. Finally, the detected current around $2 f_{L O}$ is:

$$
\begin{aligned}
& i_{p d_{2} 2 L O}(t)=\frac{\Re t_{f f}}{L_{f} L} 2 J_{1}^{2}\left(m_{L O}\right) \\
& {\left[I^{2}\left(t+\beta_{1} L+\beta_{2} L\left(\Omega_{1}-\Omega_{0}\right)\right) \cos \left(2 \omega_{L O}\left(t+\beta_{1} L+\beta_{2} L\left(\Omega_{1}-\Omega_{0}\right)\right)\right)\right.} \\
& \left.+Q^{2}\left(t+\beta_{1} L+\beta_{2} L\left(\Omega_{2}-\Omega_{0}\right)\right) \cos \left(2 \omega_{L O}\left(t+\beta_{1} L+\beta_{2} L\left(\Omega_{2}-\Omega_{0}\right)\right)\right)\right]
\end{aligned}
$$

$\mathrm{I}(\mathrm{t})$ and its $2 \mathrm{LO}$ carrier are delayed according to $\tau_{1}=-\beta_{1} L-\beta_{2} L\left(\Omega_{1}-\Omega_{0}\right)$ whereas $\mathrm{Q}(\mathrm{t})$ and its $2 \mathrm{LO}$ carrier are delayed according to $\tau_{2}=-\beta_{I} L-\beta_{2} L\left(\Omega_{2}-\Omega_{0}\right)$, that is to say, the $\mathrm{Q}$ component is delayed relative to the I carrier by

$$
\tau_{21}=\tau_{2}-\tau_{1}=-\beta_{2} L\left(\Omega_{2}-\Omega_{1}\right)
$$

In this case the quadrature condition will be: 


$$
\tau_{21}=-\beta_{2} L\left(\Omega_{2}-\Omega_{1}\right)=\frac{\tau_{2 L O}}{4}=\frac{1}{4 f_{2 L O}}=\frac{1}{8 f_{L O}}
$$

In this case, the $2 \mathrm{LO}$ component will be made of a fixed $2 \mathrm{LO}$ carrier and an ideal QAM signal,

$$
\begin{aligned}
& i_{p d_{-} L O}(t) \approx \frac{\Re t_{f f}}{L_{f} L} 2 J_{1}^{2}\left(m_{L O}\right) \\
& {\left[P \sqrt{2} \cos \left(2 \omega_{L O} t-\frac{\pi}{4}\right)+\eta\left[x_{I}(t) \cos \left(2 \omega_{L O} t\right)+x_{Q}(t) \sin \left(2 \omega_{L O} t\right)\right]\right]}
\end{aligned}
$$

\section{C.5. Simplified PVM model without LO leakage}

In order to avoid the undesired LO level present at the output of the PVM a third laser has been included in the PVM scheme as shown in ¡Error! No se encuentra el orige n de la referencia. The signals generated at each laser can be expressed as:

$$
\begin{gathered}
E_{\text {laser } 1}=\sqrt{2\left(P_{1}+\eta_{1} x_{I}(t)\right)} e^{j\left(\Omega_{1} t+\varphi_{1}\right)} \\
E_{\text {laser } 2}=\sqrt{2\left(P_{2}+\eta_{2} x_{Q}(t)\right)} e^{j\left(\Omega_{2} t+\varphi_{2}\right)} \\
E_{\text {laser } 3}=\sqrt{2\left(P_{3}\right)} e^{j\left(\Omega_{3} t+\phi_{3}\right)}
\end{gathered}
$$

where $P_{i}$ is the output optical power at laser $i$ corresponding to the laser bias point and $x_{I}(t)$ and $x_{Q}(t)$ are the base-band data signals.

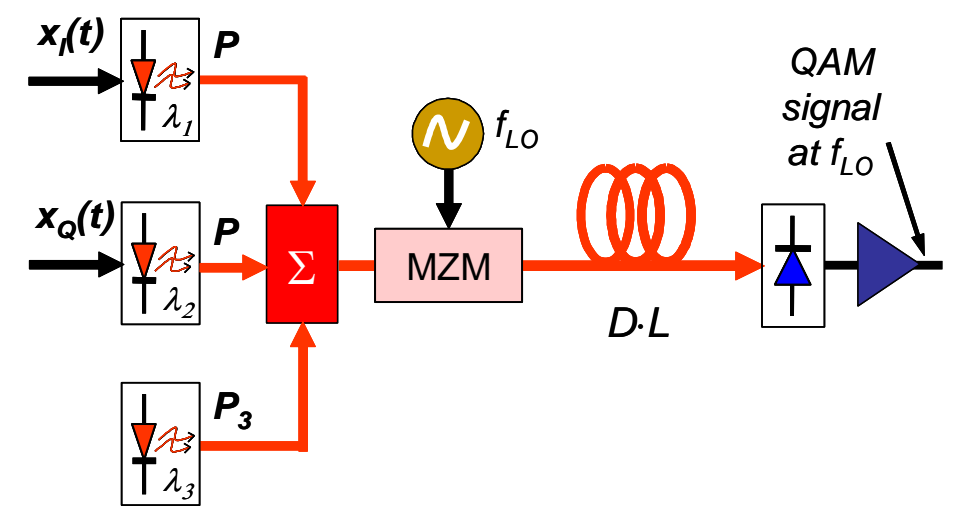

Figure C.4. B lock diagram a PVM with a third laser to avoid LO leakage.

At the MZM input: 


$$
\begin{aligned}
& E_{\text {laser }}= \\
& \sqrt{\frac{2\left(P_{1}+\eta_{1} x_{I}(t)\right)}{L_{1}}} e^{j\left(\Omega_{1} t+\varphi_{1}^{\prime}\right)}+\sqrt{\frac{2\left(P_{2}+\eta_{2} x_{Q}(t)\right)}{L_{2}}} e^{j\left(\Omega_{2} t+\varphi_{2}^{\prime}\right)}+\sqrt{\frac{2 P_{3}}{L_{3}}} e^{j\left(\Omega_{3} t+\varphi_{3}^{\prime}\right)}
\end{aligned}
$$

At the MZM output, assuming biasing at QB $\left(t_{f f}\right.$ are the MZM insertion losses when the modulator is biased at MATB, ideally $\left.t_{f f}=1\right)$. The MZM behaviour is assumed to be frequency independent with respect to $\Omega$.

$$
\begin{aligned}
& E_{m z m}(t)=\left[\sqrt{2 \frac{t_{f f}}{L_{3}} P_{3}} e^{j\left(\Omega_{3} t+\varphi_{3}^{\prime \prime}\right)}+\sqrt{2 \frac{t_{f f}}{L_{1}}\left(P_{1}+\eta_{1} x_{I}(t-\tau)\right)} e^{j\left(\Omega_{1} t+\varphi_{1}^{\prime \prime}\right)}\right. \\
& \left.+\sqrt{2 \frac{t_{f f}}{L_{2}}\left(P_{2}+\eta_{2} x_{Q}(t-\tau)\right)} e^{j\left(\Omega_{2} t+\varphi_{2}^{\prime \prime}\right)}\right] \sum_{n=-\infty}^{\infty} J_{n}\left(m_{L O}\right) C_{D C}(n) e^{j\left(n \omega_{L O} t+n \varphi_{L O}\right)}
\end{aligned}
$$

where $C_{D C}(n)$ and $m_{L O}$ as defined in C.1.

If only first terms are considered and the relation $J_{-1}\left(m_{L O}\right)=-J_{1}\left(m_{L O}\right)$ is applied:

$$
\begin{aligned}
& E_{m z m}(t) \approx\left[\sqrt{\frac{t_{f f}}{L_{3}} P_{3}} e^{j\left(\Omega_{3} t+\varphi_{3}^{\prime \prime}\right)}+\sqrt{\frac{t_{f f}}{L_{1}}\left(P_{1}+\eta_{1} x_{I}(t-\tau)\right)} e^{j\left(\Omega_{1} t+\varphi_{1}^{\prime \prime}\right)}\right. \\
& \left.+\sqrt{\frac{t_{f f}}{L_{2}}\left(P_{2}+\eta_{2} x_{Q}(t-\tau)\right)} e^{j\left(\Omega_{2} t+\varphi_{2}^{\prime \prime}\right)}\right]\left[J_{0}\left(m_{L O}\right) \pm 2 J_{1}\left(m_{L O}\right) \cos \left(\omega_{L O} t+\varphi_{L O}\right)\right]
\end{aligned}
$$

Assuming a simplified link that induces just a $\pi / 2$ radians phase shift at $f_{L O}$ between $\Omega_{1}$ and $\Omega_{2}$ with no further effect on $x_{I}(t), x_{Q}(t)$ or RF carrier; that is to say $\Omega_{1}-\Omega_{2}$ is quite greater than the frequency contents of BB signals or LO. At the same time a $5 \pi / 4$ radians phase shift will be induced at $f_{L O}$ between $\Omega_{1}$ and $\Omega_{3}$ if $\Omega_{3}-\Omega_{1} \approx 2.5\left(\Omega_{2}-\Omega_{1}\right)^{21}$.

${ }^{21}$ To be more precise, the actual relation should be $\Delta \tau_{31}=2.5 \Delta \tau_{21}$ where $\Delta \tau_{31}$ and $\Delta \tau_{21}$ are the relative delay between the respective carriers due to the dispersion of the fibre link). 


$$
\begin{aligned}
& E_{\text {link }}(t) \approx \sqrt{\frac{t_{f f}}{L_{1} L_{f}}\left(P_{1}+\eta_{1} x_{I}\left(t-\tau^{\prime}\right)\right)} e^{j\left(\Omega_{1} t+\varphi_{i}^{\prime}\right)}\left[J_{0}\left(m_{L O}\right) \pm 2 J_{1}\left(m_{L O}\right) \cos \left(\omega_{L O} t+\varphi_{L O}^{\prime}\right)\right] \\
& +\sqrt{\frac{t_{f f}}{L_{2} L_{f}}\left(P_{2}+\eta_{2} x_{Q}\left(t-\tau^{\prime}-\frac{1}{4 f_{L O}}\right)\right)} e^{j\left(\Omega_{2} t+\varphi_{2}^{\prime}\right)}\left[J_{0}\left(m_{L O}\right) \pm 2 J_{1}\left(m_{L O}\right) \cos \left(\omega_{L O} t-\frac{\pi}{2}+\varphi_{L O}^{\prime}\right)\right] \\
& +\sqrt{\frac{t_{f f}}{L_{3} L_{f}} P_{3} e^{j\left(\Omega_{3} t+\varphi_{3}^{\prime}\right)}}\left[J_{0}\left(m_{L O}\right) \pm 2 J_{1}\left(m_{L O}\right) \cos \left(\omega_{L O} t-5 \frac{\pi}{4}+\varphi_{L O}^{\prime}\right)\right]
\end{aligned}
$$

Finally, the photocurrent can be calculated as:

$$
\begin{aligned}
& i_{p d}(t) \approx \frac{\Re}{2} \frac{t_{f f}}{L_{f}}\left[\left(\frac{P_{1}}{L_{1}}+\frac{\eta_{1}}{L_{1}} x_{I}\left(t-\tau^{\prime}\right)\right)\left[J_{0}\left(m_{L O}\right) \pm 2 J_{1}\left(m_{L O}\right) \cos \left(\omega_{L O} t+\varphi_{L O}^{\prime}\right)\right]^{2}\right. \\
& +\left(\frac{P_{2}}{L_{2}}+\frac{\eta_{2}}{L_{2}} x_{Q}\left(t-\tau^{\prime}-\frac{1}{4 f_{L O}}\right)\right)\left[J_{0}\left(m_{L O}\right) \pm 2 J_{1}\left(m_{L O}\right) \cos \left(\omega_{L O} t-\frac{\pi}{2}+\varphi_{L O}^{\prime}\right)\right]^{2} \\
& \left.+\frac{P_{3}}{L_{2}}\left[J_{0}\left(m_{L O}\right) \pm 2 J_{1}\left(m_{L O}\right) \cos \left(\omega_{L O} t-5 \frac{\pi}{4}+\varphi_{L O}^{\prime}\right)\right]^{2}\right]
\end{aligned}
$$

where the $\pm\left(\Omega_{i} \pm \Omega_{j}\right)(i \neq j)$ terms have been neglected.

The photocurrent $\mathrm{DC}$ value is:

$$
i_{p d_{-} D C} \approx \frac{\Re}{2} \frac{t_{f f}}{L_{f} L}(2+\sqrt{2}) P\left[J_{0}^{2}\left(m_{L O}\right)+2 J_{1}^{2}\left(m_{L O}\right)\right]
$$

when $P=P_{1}=P_{2}, P_{3}=P(2)^{1 / 2}, L=L_{1}=L_{2}=L_{3}$.

If the terms at $\mathrm{DC}, \mathrm{BB}$ and $2 f_{L O}$ terms are neglected, the detected signal around LO will be:

$$
\begin{aligned}
i_{p d-L O}(t) \approx & \pm \frac{\mathfrak{R} t_{f f}}{L_{f} L} 2 J_{1}\left(m_{L O}\right) J_{0}\left(m_{L O}\right) \\
& {\left[\sqrt{2} P \cos \left(\omega_{L O} t-\frac{\pi}{4}\right)+\sqrt{2} P \cos \left(\omega_{L O} t-\frac{5}{4} \pi\right)\right.} \\
& \left.+\eta x_{I}(t) \cos \left(\omega_{L O} t\right)+\eta x_{Q}\left(t-\frac{1}{4 f_{L O}}\right) \sin \left(\omega_{L O} t\right)\right]
\end{aligned}
$$

where it has been considered that $P=P_{1}=P_{2}, P_{3}=P(2)^{1 / 2}, L=L_{1}=L_{2}=L_{3}$ and $\eta=\eta_{1}=\eta_{2}$; and common delay/phase terms like $\tau^{\prime}$ or $\phi_{L O}$ ': have been neglected. 
Eq (C.65) ban be simplified as:

$$
\begin{aligned}
i_{p d-L O}(t) \approx & \pm \frac{\Re t_{f f}}{L_{f} L} 2 J_{1}\left(m_{L O}\right) J_{0}\left(m_{L O}\right) \eta \\
& {\left[x_{I}(t) \cos \left(\omega_{L O} t\right)+x_{Q}\left(t-\frac{1}{4 f_{L O}}\right) \sin \left(\omega_{L O} t\right)\right] }
\end{aligned}
$$

where it can be seen that the LO tone term has disappeared and the quadrature condition between the I and Q terms is fulfilled.

The detected signal power assuming a load resistance equal to $R_{L}$ :

$$
\begin{aligned}
P_{p d-\text { data }} & =\frac{1}{2}\left[\frac{\mathfrak{R} t_{f f}}{L L_{f}} 2 J_{1}\left(m_{L O}\right) J_{0}\left(m_{L O}\right) \eta\right]^{2}\left(P_{I}+P_{Q}\right) R_{L} \\
& =2 \eta^{2}\left(P_{I}+P_{Q}\right)\left[\frac{\mathfrak{R} t_{f f}}{L L_{f}} J_{1}\left(m_{L O}\right) J_{0}\left(m_{L O}\right)\right]^{2} R_{L}
\end{aligned}
$$

where $P_{I}=\left\langle x_{I}^{2}(t)\right\rangle$ and $P_{Q}=\left\langle x_{Q}^{2}(t)\right\rangle$.

If expression (C.9) and (C.64) are compared, it can be seen that the DC term has been modified by the insertion of the third laser. This new factor increases the DC current by a factor of 1.7 which increase the RIN noise by $4.6 \mathrm{~dB}$ and the shot noise by $2.3 \mathrm{~dB}$. Depending on the dominating noise component, the effect of the addition of the third laser will reduce the SNR by a factor of $0 \mathrm{~dB}$ (thermal noise dominates) up to $4.6 \mathrm{~dB}$ in the worst case (RIN dominates).

\section{C.6. Complete 3-lasers PVM model including a second-order dispersive link (QB biasing)}

In this section, the previous assumption of a simplified link inducing $\pi / 2$ radians and $5 \pi / 4$ radians phase shifts between the optical carriers is proved by taking into the chromatic dispersion of the optical link up to its second order.

Assuming $L=L_{1}=L_{2}=L_{3}, \quad P=P_{1}=P_{2}$ and $\eta=\eta_{1}=\eta_{2}$, equation (C.61) can be expressed as: 


$$
\begin{aligned}
& E_{m z m}(t) \approx\left[J_{0}\left(m_{L O}\right) \pm 2 J_{1}\left(m_{L O}\right) \cos \left(\omega_{L O} t+\varphi_{L O}\right)\right] \sqrt{\frac{t_{f f}}{L}} \\
& {\left[\sqrt{P+\eta x_{I}(t-\tau)} e^{j\left(\Omega_{1} t+\varphi_{1}^{\prime \prime}\right)}+\sqrt{P+\eta x_{Q}(t-\tau)} e^{j\left(\Omega_{2} t+\varphi_{2}^{\prime \prime}\right)}+\sqrt{P_{3}} e^{j\left(\Omega_{3} t+\varphi_{3}^{\prime \prime}\right)}\right]}
\end{aligned}
$$

which equals to:

$$
\begin{aligned}
E_{m z m}(t) \approx A\left[B \pm C \cos \left(\omega_{L O} t+\varphi_{L O}\right)\right] \\
{\left[I(t) e^{j\left(\Omega_{1} t+\varphi_{1}^{\prime \prime}\right)}+Q(t) e^{j\left(\Omega_{2} t+\varphi_{2}^{\prime \prime}\right)}+\sqrt{P_{3}} e^{j\left(\Omega_{3} t+\varphi_{3}^{\prime \prime}\right)}\right] }
\end{aligned}
$$

where $A, B, C, I(t)$ and $Q(t)$ from (C.34).

Equation (C.69) can be expressed as:

$$
\begin{aligned}
E_{m z m}(t) \approx A\left[B \pm \frac{C}{2} e^{j\left(\omega_{L O} t+\varphi_{L O}\right)} \pm \frac{C}{2} e^{-j\left(\omega_{L O} t+\varphi_{L O}\right)}\right] \\
{\left[I(t) e^{j\left(\Omega_{1} t+\varphi_{1}^{\prime \prime}\right)}+Q(t) e^{j\left(\Omega_{2} t+\varphi_{2}^{\prime \prime}\right)}+\sqrt{P_{3}} e^{j\left(\Omega_{3} t+\varphi_{3}^{\prime \prime}\right)}\right] }
\end{aligned}
$$

assuming that the electrical and optical phase play no role, neither the sign ambiguity,

$$
\begin{aligned}
E_{m z m}(t) \approx & A\left[B+\frac{C}{2} e^{j \omega_{L O} t}+\frac{C}{2} e^{-j \omega_{L O} t}\right]\left[I(t) e^{j \Omega_{1} t}+Q(t) e^{j \Omega_{2} t}+\sqrt{P_{3}} e^{j \Omega_{3} t}\right] \\
& =A I(t)\left[B e^{j \Omega_{1} t}+\frac{C}{2} e^{j\left(\Omega_{1}+\omega_{L O}\right) t}+\frac{C}{2} e^{j\left(\Omega_{1}-\omega_{L O}\right) t}\right] \\
& +A Q(t)\left[B e^{j \Omega_{2} t}+\frac{C}{2} e^{j\left(\Omega_{2}+\omega_{L O}\right) t}+\frac{C}{2} e^{j\left(\Omega_{2}-\omega_{L O}\right) t}\right] \\
& +A \sqrt{P_{3}}\left[B e^{j \Omega_{3} t}+\frac{C}{2} e^{j\left(\Omega_{3}+\omega_{L O}\right) t}+\frac{C}{2} e^{j\left(\Omega_{3}-\omega_{L O}\right) t}\right]
\end{aligned}
$$

After the length of the dispersive link, $L$, and neglecting the effect of dispersion on $I(t)$ or $Q(t)$, that is to say assuming their frequency content is small compared to the dispersion of the link, the expression (C.73) can be obtained. However, the dispersion of the link is delaying $Q(t)$ respect to $I(t)$ in the same way as the corresponding LO terms, the only way to model this is assuming that $I(t)$ and $Q(t)$ are delayed according to the group delays at $\Omega_{1}$ and $\Omega_{2}$ ): 


$$
\begin{aligned}
& I^{\prime}(t)=I\left(t+\beta_{1} L+\beta_{2} L\left(\Omega_{1}-\Omega_{0}\right)\right) \\
& Q^{\prime}(t)=Q\left(t+\beta_{1} L+\beta_{2} L\left(\Omega_{2}-\Omega_{0}\right)\right) \\
& E_{\text {link }}(t) \approx E_{m z m}(t) e^{j \beta(\Omega) L} \\
& =A^{\prime} I^{\prime}(t) e^{j \beta_{0} L}\left[\begin{array}{l}
B e^{j \Omega_{1} t} e^{j \beta_{1} L\left(\Omega_{1}-\Omega_{0}\right)} e^{j \frac{\beta_{2} L}{2}\left(\Omega_{1}-\Omega_{0}\right)^{2}} \\
+\frac{C}{2} e^{j\left(\Omega_{1}+\omega_{L O}\right) t} e^{j \beta_{1} L\left(\Omega_{1}+\omega_{L O}-\Omega_{0}\right)} e^{j \frac{\beta_{2} L}{2}\left(\Omega_{1}+\omega_{L O}-\Omega_{0}\right)^{2}} \\
+\frac{C}{2} e^{j\left(\Omega_{1}-\omega_{L O}\right) t} e^{j \beta_{1} L\left(\Omega_{1}-\omega_{L O}-\Omega_{0}\right)} e^{j \frac{\beta_{2} L}{2}\left(\Omega_{1}-\omega_{L O}-\Omega_{0}\right)^{2}}
\end{array}\right] \\
& +A^{\prime} Q^{\prime}(t) e^{j \beta_{0} L}\left[\begin{array}{l}
B e^{j \Omega_{2} t} e^{j \beta_{1} L\left(\Omega_{2}-\Omega_{0}\right)} e^{j \frac{\beta_{2} L}{2}\left(\Omega_{2}-\Omega_{0}\right)^{2}} \\
+\frac{C}{2} e^{j\left(\Omega_{2}+\omega_{L O}\right) t} e^{j \beta_{1} L\left(\Omega_{2}+\omega_{L O}-\Omega_{0}\right)} e^{j \frac{\beta_{2} L}{2}\left(\Omega_{2}+\omega_{L O}-\Omega_{0}\right)^{2}} \\
+\frac{C}{2} e^{j\left(\Omega_{2}-\omega_{L O}\right) t} e^{j \beta_{1} L\left(\Omega_{2}-\omega_{L O}-\Omega_{0}\right)} e^{j \frac{\beta_{2} L}{2}\left(\Omega_{2}-\omega_{L O}-\Omega_{0}\right)^{2}}
\end{array}\right] \\
& +A^{\prime} \sqrt{P_{3}} e^{j \beta_{0} L}\left[\begin{array}{l}
B e^{j \Omega_{3} t} e^{j \beta_{1} L\left(\Omega_{3}-\Omega_{0}\right)} e^{j \frac{\beta_{2} L}{2}\left(\Omega_{3}-\Omega_{0}\right)^{2}} \\
+\frac{C}{2} e^{j\left(\Omega_{3}+\omega_{L O}\right) t} e^{j \beta_{1} L\left(\Omega_{3}+\omega_{L O}-\Omega_{0}\right)} e^{j \frac{\beta_{2} L}{2}\left(\Omega_{3}+\omega_{L O}-\Omega_{0}\right)^{2}} \\
+\frac{C}{2} e^{j\left(\Omega_{3}-\omega_{L O}\right) t} e^{j \beta_{1} L\left(\Omega_{3}-\omega_{L O}-\Omega_{0}\right)} e^{j \frac{\beta_{2} L}{2}\left(\Omega_{3}-\omega_{L O}-\Omega_{0}\right)^{2}}
\end{array}\right]
\end{aligned}
$$

where $A^{\prime}=\sqrt{t_{f f} /\left(L_{f} L\right)}$.

After some algebra, following expression can be obtained for the optical field impinging the photoreceiver: 


$$
\begin{aligned}
E_{\text {link }}(t) \approx & A^{\prime} I^{\prime}(t) e^{j \beta_{0} L} e^{j \Omega_{1} t} e^{j \beta_{1} L\left(\Omega_{1}-\Omega_{0}\right)} e^{j \frac{\beta_{2} L}{2}\left(\Omega_{1}-\Omega_{0}\right)^{2}} \\
& {\left[B+e^{j \frac{\beta_{2} L}{2} \omega_{L O}^{2}} C \cos \left(\omega_{L O}\left(t+\beta_{1} L\right)+\beta_{2} L\left(\Omega_{1}-\Omega_{0}\right) \omega_{L O}\right)\right] } \\
& +A^{\prime} Q^{\prime}(t) e^{j \beta_{0} L} e^{j \Omega_{2} t} e^{j \beta_{1} L\left(\Omega_{2}-\Omega_{0}\right)} e^{j \frac{\beta_{2} L}{2}\left(\Omega_{2}-\Omega_{0}\right)^{2}} \\
& {\left[B+e^{j \frac{\beta_{2} L}{2} \omega_{L O}^{2}} C \cos \left(\omega_{L O}\left(t+\beta_{1} L\right)+\beta_{2} L\left(\Omega_{2}-\Omega_{0}\right) \omega_{L O}\right)\right] } \\
& +A^{\prime} \sqrt{P_{3}} e^{j \beta_{0} L} e^{j \Omega_{3} t} e^{j \beta_{1} L\left(\Omega_{3}-\Omega_{0}\right)} e^{j \frac{\beta_{2} L}{2}\left(\Omega_{3}-\Omega_{0}\right)^{2}} \\
& {\left[B+e^{j \frac{\beta_{2} L}{2} \omega_{L O}^{2}} C \cos \left(\omega_{L O}\left(t+\beta_{1} L\right)+\beta_{2} L\left(\Omega_{3}-\Omega_{0}\right) \omega_{L O}\right)\right] }
\end{aligned}
$$

And the photocurrent can be expressed as:

$$
\begin{aligned}
& i_{p d}(t)=\frac{\mathfrak{R} E_{\text {link }} E_{\text {link }}^{*}=\frac{\mathfrak{R} A^{\prime 2}}{2}}{2}\left[\begin{array}{l}
I^{\prime 2}(t)\left[\begin{array}{l}
2 B C \cos \left(\frac{\beta_{2} L}{2} \omega_{L O}^{2}\right) \cos \left(\omega_{L O}\left(t+\beta_{1} L\right)+\beta_{2} L\left(\Omega_{1}-\Omega_{0}\right) \omega_{L O}\right) \\
+B^{2}+C^{2} \cos ^{2}\left(\omega_{L O}\left(t+\beta_{1} L\right)+\beta_{2} L\left(\Omega_{1}-\Omega_{0}\right) \omega_{L O}\right)
\end{array}\right] \\
+Q^{\prime 2}(t)\left[\begin{array}{l}
2 B C \cos \left(\frac{\beta_{2} L}{2} \omega_{L O}^{2}\right) \cos \left(\omega_{L O}\left(t+\beta_{1} L\right)+\beta_{2} L\left(\Omega_{2}-\Omega_{0}\right) \omega_{L O}\right) \\
+B^{2}+C^{2} \cos ^{2}\left(\omega_{L O}\left(t+\beta_{1} L\right)+\beta_{2} L\left(\Omega_{2}-\Omega_{0}\right) \omega_{L O}\right)
\end{array}\right] \\
+P_{3}\left[\begin{array}{l}
\left.2 B C \cos \left(\frac{\beta_{2} L}{2} \omega_{L O}^{2}\right) \cos \left(\omega_{L O}\left(t+\beta_{1} L\right)+\beta_{2} L\left(\Omega_{3}-\Omega_{0}\right) \omega_{L O}\right)\right] \\
+B^{2}+C^{2} \cos ^{2}\left(\omega_{L O}\left(t+\beta_{1} L\right)+\beta_{2} L\left(\Omega_{3}-\Omega_{0}\right) \omega_{L O}\right)
\end{array}\right]
\end{array}\right]
\end{aligned}
$$

where the $\pm\left(\Omega_{\mathrm{i}} \pm \Omega_{\mathrm{i}}\right)(i \neq j)$ terms have been neglected. Finally, the DC detected current is:

$$
i_{p d_{-} D C}(t)=\frac{\mathfrak{R}\left(2 P+P_{3}\right)}{2 L_{t o t}}\left[J_{0}^{2}\left(m_{L O}\right)+2 J_{1}^{2}\left(m_{L O}\right)\right]
$$

And the detected term around $f_{L O}$ is: 


$$
\begin{aligned}
& i_{p d_{-} L O}(t)=\mathfrak{R} A^{\prime 2} B C \cos \left(\frac{\beta_{2} L}{2} \omega_{L O}^{2}\right) \\
& {\left[\begin{array}{l}
I^{2}\left(t+\beta_{1} L+\beta_{2} L\left(\Omega_{1}-\Omega_{0}\right)\right) \cos \left(\omega_{L O}\left(t+\beta_{1} L+\beta_{2} L\left(\Omega_{1}-\Omega_{0}\right)\right)\right) \\
+Q^{2}\left(t+\beta_{1} L+\beta_{2} L\left(\Omega_{2}-\Omega_{0}\right)\right) \cos \left(\omega_{L O}\left(t+\beta_{1} L+\beta_{2} L\left(\Omega_{2}-\Omega_{0}\right)\right)\right) \\
+P_{3} \cos \left(\omega_{L O}\left(t+\beta_{1} L+\beta_{2} L\left(\Omega_{3}-\Omega_{0}\right)\right)\right)
\end{array}\right]}
\end{aligned}
$$

According to this result, $I(t)$ and its LO carrier are delayed according to $\tau_{1}=-\beta_{1} L-\beta_{2} L\left(\Omega_{1}-\Omega_{0}\right)$ whereas $Q(t)$ and its LO carrier are delayed according to $\tau_{2}=-\beta_{1} L-\beta_{2} L\left(\Omega_{2}-\Omega_{0}\right)$ and $\Omega_{3}$ is delayed according to $\tau_{3}=-\beta_{1} L-\beta_{2} L\left(\Omega_{3}-\Omega_{0}\right)$, that is to say, the $\mathrm{Q}$ component is delayed relative to the I carrier by

$$
\tau_{21}=\tau_{2}-\tau_{1}=-\beta_{2} L\left(\Omega_{2}-\Omega_{1}\right)
$$

which is a positive value for wavelengths in the anomalous region of th standard single-mode fibre when $\Omega_{2}>\Omega_{1}$. At the same time the third laser is delayed relative to $\Omega_{1}$ as:

$$
\tau_{31}=\tau_{3}-\tau_{1}=-\beta_{2} L\left(\Omega_{3}-\Omega_{1}\right)
$$

and substituting the values,

$$
\begin{aligned}
i_{p d_{-} L O}(t) & =\Re \frac{t_{f f}}{L_{f} L} J_{0}\left(m_{L O}\right) 2 J_{1}\left(m_{L O}\right) \cos \left(\frac{\beta_{2} L}{2} \omega_{L O}^{2}\right) \\
& {\left[\begin{array}{c}
{\left[P+\eta x_{I}(t)\right] \cos \left(\omega_{L O} t\right)} \\
+\left[P+\eta x_{Q}\left(t-\tau_{21}\right)\right] \cos \left(\omega_{L O}\left(t-\tau_{21}\right)\right) \\
+P_{3} \cos \left(\omega_{L O}\left(t-\tau_{31}\right)\right)
\end{array}\right] }
\end{aligned}
$$

